\title{
Anatomical and physiological properties of the superior paraolivary nucleus in the rat
}

Randy Joseph Kulesza Jr.

West Virginia University

Follow this and additional works at: https://researchrepository.wvu.edu/etd

\section{Recommended Citation}

Kulesza, Randy Joseph Jr., "Anatomical and physiological properties of the superior paraolivary nucleus in the rat" (2002). Graduate Theses, Dissertations, and Problem Reports. 1630.

https://researchrepository.wvu.edu/etd/1630

This Dissertation is protected by copyright and/or related rights. It has been brought to you by the The Research Repository @ WVU with permission from the rights-holder(s). You are free to use this Dissertation in any way that is permitted by the copyright and related rights legislation that applies to your use. For other uses you must obtain permission from the rights-holder(s) directly, unless additional rights are indicated by a Creative Commons license in the record and/ or on the work itself. This Dissertation has been accepted for inclusion in WVU Graduate Theses, Dissertations, and Problem Reports collection by an authorized administrator of The Research Repository @ WVU.

For more information, please contact researchrepository@mail.wvu.edu. 


\title{
Anatomical and Physiological Properties of the Superior Paraolivary Nucleus in the Rat
}

\author{
Randy J. Kulesza Jr. \\ Dissertation submitted to the \\ School of Medicine \\ at West Virginia University \\ in partial fulfillment of the requirements \\ for the degree of \\ Doctor of Philosophy \\ in \\ Anatomy \\ Albert Berrebi, Ph.D., Chair \\ Aric Agmon, Ph.D. \\ James Culberson, Ph.D \\ Richard Dey, Ph.D. \\ George Spirou, Ph.D. \\ Department of Neurobiology and Anatomy \\ Morgantown, West Virginia \\ 2002
}

Keywords: superior olivary complex, periolivary, inferior colliculus, inhibition, inhibitory rebound 


\title{
ABSTRACT \\ Anatomical and Physiological Properties of the Superior Paraolivary Nucleus in the Rat
}

\author{
Randy J. Kulesza Jr.
}

The superior paraolivary nucleus (SPON) is a group of neurons located within the superior olivary complex, a constellation of brainstem nuclei involved in auditory processing. The major inputs to the SPON arise from the contralateral ear and SPON axons target primarily the ipsilateral inferior colliculus. However, little is known regarding the neurochemical phenotypes present in the SPON and how these neurons respond to auditory stimuli. Understanding the neurochemical and physiological properties of the constituent neurons will provide insight into the functional role of the SPON and will contribute to our understanding of the neural circuitry involved in hearing. Immunocytochemical, stereological, physiological and pharmacological techniques were used to characterize SPON neurons in the rat. The presence of inhibitory neurotransmitters was investigated with immunocytochemistry and provides evidence that the SPON contains a morphologically homogeneous population of GABAergic neurons and further that these neurons receive a robust inhibitory innervation in the form of glycinergic and GABAergic inputs. Stereological estimates of total neuron number in eighteen subcortical auditory nuclei provide evidence that the SPON is a prominent brainstem cell group and a major source of ascending inhibition to the inferior colliculus. Extracellular in vivo recordings provide evidence that nearly all SPON neurons respond to sound played in the contralateral ear with spike activity timed to the stimulus offset and phase lock to amplitude modulations in complex sounds. Pharmacologically blocking glycinergic input abolished the offset response (indicating that offset activity is a rebound from glycinergic inhibition); blockade of glycinergic and GABAergic input simultaneously, resulted in broader receptive fields and reduced phase locking capabilities. Taken together, these data indicate the rat SPON is a prominent auditory cell group that provides GABAergic inhibition to the ipsilateral inferior colliculus indicating the sound offset. GABAergic inhibition has been implicated in numerous aspects of auditory physiology, including sound localization and sensitivity to stimulus duration. Therefore, the SPON plays an important role in auditory processing and offset inhibition may be involved in processing complex sounds and in creating sensitivity to stimulus duration, both important features of animal and human communication. 


\section{Dedication}

This work is dedicated to the loving memory of my mother

\section{Cynthia Kulesza}

who passed away on June 15, 2000. 


\section{Acknowledgments}

To my committee members Dr. Culberson, Dr. Dey and Dr. Agmon, I would like to thank you for your support, guidance, encouragement and for always making yourselves available. Dr. Agmon, I sincerely thank you for you thoughtful comments on the GAD, stereology and physiology manuscripts.

Dr. Spirou, your instruction and guidance during the physiology portions of my dissertation were essential to this project. This work could not have been completed without your insight. Your thoughtful critiques of our manuscripts were appreciated and only served to improve them.

I am indebted to Dr. Pope for allowing me to serve as a teaching assistant in the medical and dental gross anatomy courses. Some of my most enjoyable experiences in graduate school occurred while I was teaching in gross lab. My lectures to the dental students have proved to be a valuable and rewarding experience that allowed me to grow as a teacher, for this I am thankful.

To my mentor, Dr. Berrebi: I think I've come a long way as a scientist, teacher and a person in the last six years; you've helped me along in each of these. You've taught me many valuable lessons, most importantly how this whole science thing works. Looking back, I can honestly say that I enjoyed working with you and I'm grateful to have had you as a mentor.

Thanks also to my Spanish colleagues and friends, Antonio Viñuela and Dr. Enrique Saldaña for your interest and involvement in the stereology project.

To my lab cohorts: Roger Bell, Janelle Grimes, Nancy Irby, Colette McNichols, Barbara Muller, Brian Pope, Kevin Rowland and Jesse Thompson thank you all for your wonderful sense of humor and for making the lab a great place to work.

To my parents, grandparents, family and friends: your support and encouragement continues to motivate me

Most of all, I'd like to thank my wife, Carol and my children Randy and Emily. The three of you have been constant sources of joy and inspiration. I could not have done this without you. 


\section{Table of Contents}

Dedication...

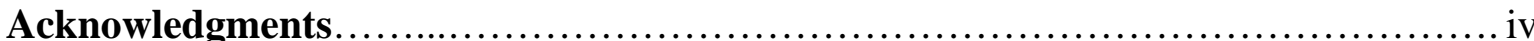

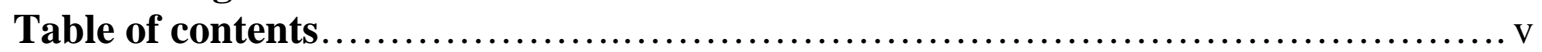

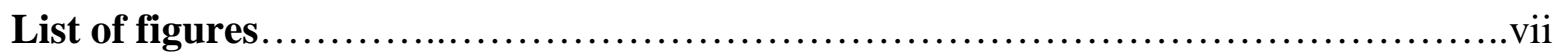

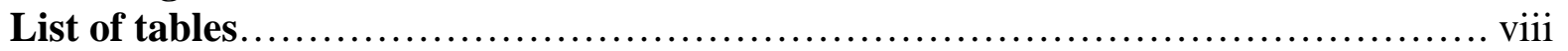

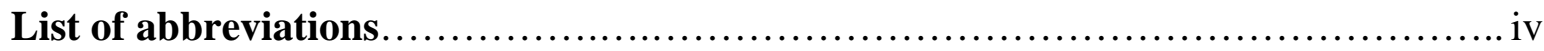

Chapter 1: Introduction

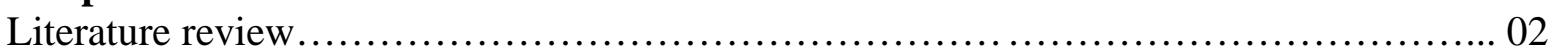

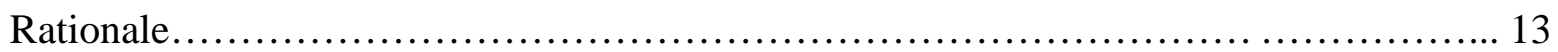

Objectives................................................................... 14

Chapter 2: The superior paraolivary nucleus is a GABAergic nucleus

Abstract........................................................................17

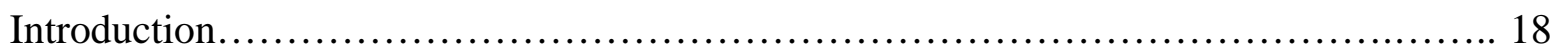

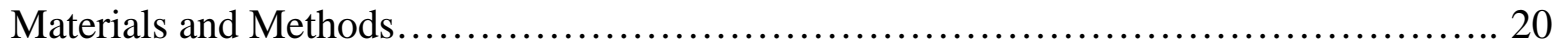

Results

Overview of glycine and GABA immunoreactivities in the SOC .................25

Quantitive analysis of glycine and GABA immunoreactivities....................26

GABA-IR and glycine-IR puncta in the SPON.............................. 27

Overview of GAD-IR in the SOC ..............................................27

GAD-IR somata in the SPON ............................................. 28

Neuronal morphology ................................................. 28

GAD-IR puncta density in the SPON ....................................... 29

Discussion...................................................................... 30

Chapter 3: Unbiased stereological estimates of neuron number in subcortical auditory nuclei of the rat

Abstract...................................................................... 49

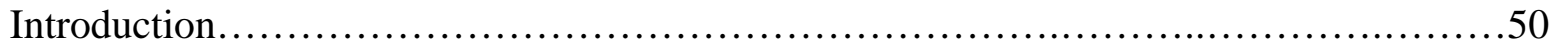

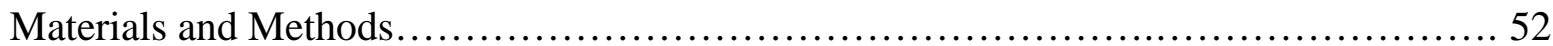

Results

Cochlear Nuclei...................................................... 55

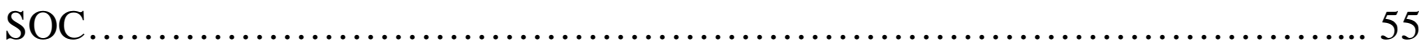

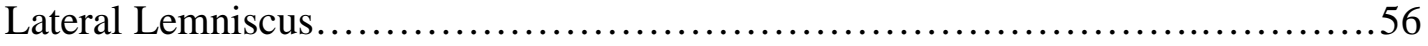

Inferior Colliculus............................................................ 57

Medial Geniculate Body................................................ 57

Three-dimensional reconstructions.......................................... 57

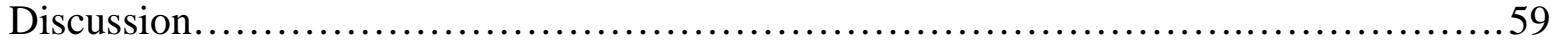

Chapter 4: Physiological response properties of neurons in the superior paraolivary nucleus neurons of the rat

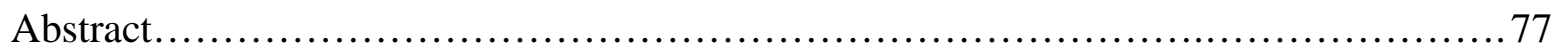

Introduction.................................................................. 78 
Materials and Methods.............................................................. 80

Results

Localization of units and tonotopic mapping of best frequencies................. 84

Responses to broad band noise and pure tones................................. 84

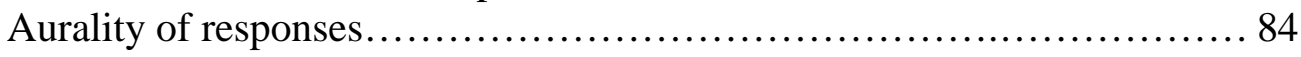

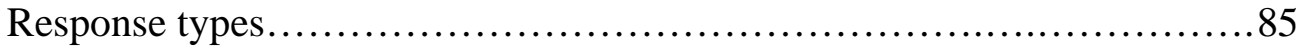

Response Latencies................................................. 86

Spontaneous activity of SPON neurons............................. 86

Receptive fields................................................... 86

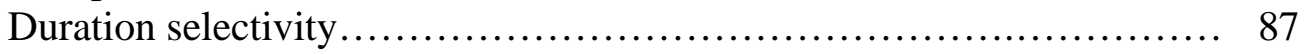

Responses to amplitude modulations............................................ 88

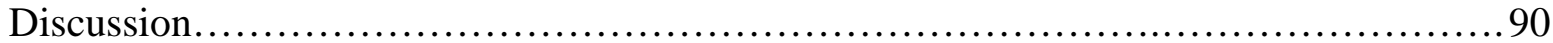

Chapter 5: The effects of GABA and glycine on response properties of superior paraolivary nucleus neurons in rat

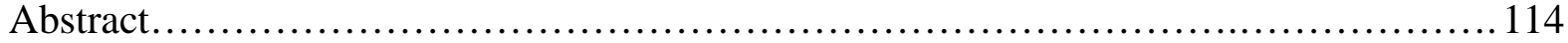

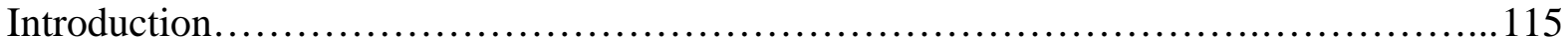

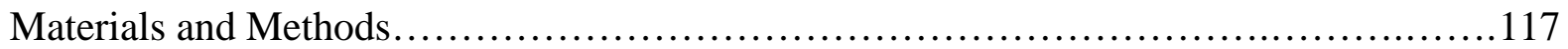

Results

Effects of blocking $\mathrm{GABA}_{\mathrm{A}}$ input........................................ 121

Effects of blocking glycinergic input....................................... 122

Effects of blocking $\mathrm{GABA}_{\mathrm{A}}$ and glycine inputs simultaneously .................... 123

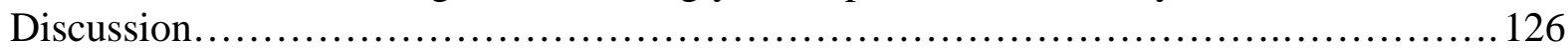

\section{Chapter 6: General Discussion}

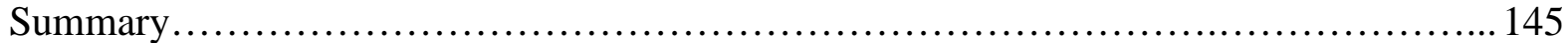

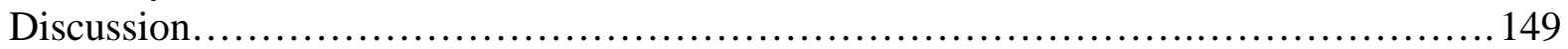

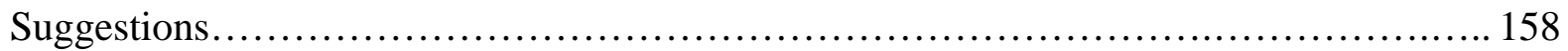

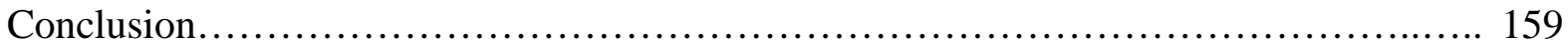

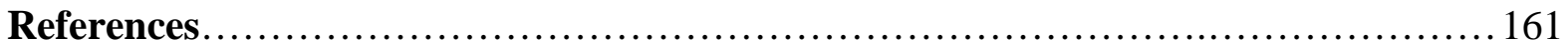

Curriculum Vitae ............................................................... 180 


\section{Index of Figures}

Chapter 2

page

2.1 Organization of the rat superior olivary complex............................... 36

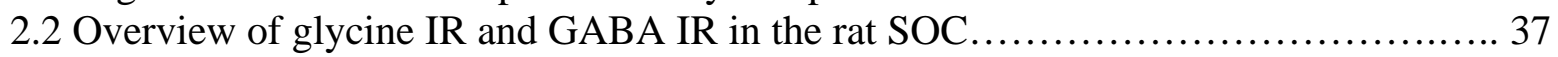

2.3 Glycine IR and GABA IR of the SPON neurons............................. 38

2.4 Assessment of glycine IR and GABA IR intensities in somata of the SOC............ 39

2.5 Immunoreactivity for glutamic acid decarboxylase (GAD) in the SOC .............. 41

2.6 GAD-65 and GAD-67 in the SOC ......................................... 42

$2.7 \mathrm{GAD}$ IR in a parasagittal section through the SOC $\ldots \ldots \ldots \ldots \ldots \ldots \ldots \ldots \ldots \ldots \ldots . \ldots \ldots$

2.8 GAD IR puncta apposed to a GAD IR neuron in the SPON ....................... 44

\section{Chapter 3}

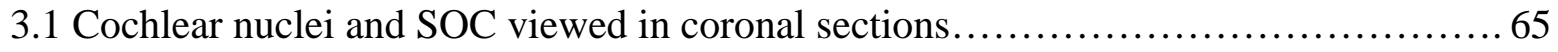

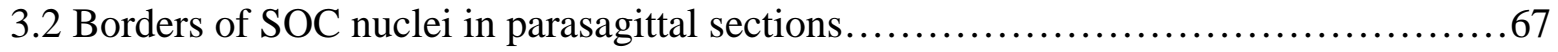

3.3 Coronal sections through he nuclei of the lateral lemniscus......................... 69

3.4 Subdivisions of the inferior colliculus in coronal section........................... 71

3.5 Subdivisions of the MGB............................................ 72

3.6 Three dimensional reconstruction of SOC nuclei............................. 73

\section{Chapter 4}

4.1 Localization of recording sites........................................... 99

4.2 Tonotopic arrangement of best frequencies in SPON ............................ 100

4.3 Single unit responses to broad band noise..................................... 101

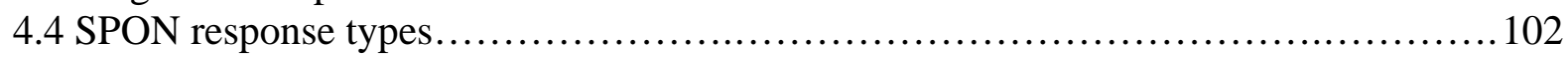

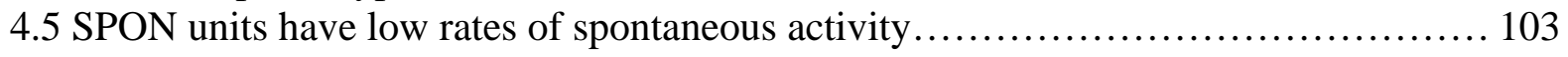

4.6 Response maps........................................................ 104

4.7 Peristimulus time histograms demonstrate that offset responses occur throughout the response map of the unit......................................... 105

4.8 SPON neurons have narrow tuning curves................................... 106

4.9 Thresholds of SPON units ................................................... 107

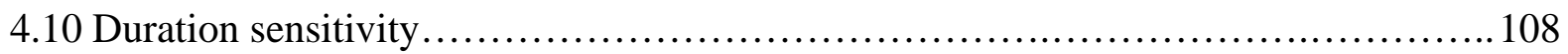

4.11 SPON unit responses to amplitude modulated tones............................. 109

4.12 Plots of discharge rates and vector strengths for SAM stimuli for the

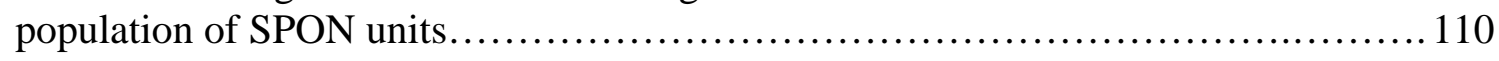

4.13 Comparision of MNTB and SPON responses to SAM .......................... 111

4.14 Responses of MNTB neurons to SAM.................................... 112

\section{Chapter 5}

5.1 Offset responses were unaffected by extracellular current injection................... 134 
5.2 Bicuculline increases offset activity of SPON neurons but had little effect

on temporal discharge pattern.................................................... 135

5.3 Bicuculline caused an increase in the number of evoked spikes.................... 136

5.4 Strychnine abolished the offset response ......................................... 137

5.5 Strychnine had a variable effect on the number of evoked spikes..................... 138

5.6 Strychnine broadened response maps........................................ 139

5.7 Bicuculline and strychnine together abolished the offset response and resulted in sustained discharges timed to the stimulus onset............................... 140

5.8 Bicuculline and strychnine together greatly increased the number of evoked

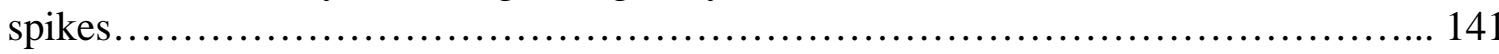

5.9 Bicuculline and strychnine caused a broadening of response maps................... 142

5.10 Application of bicuculline and strychnine resulted in lower $\mathrm{Q}_{20}$ values...............143

5.11 Blockage of GABA and glycine reduced phase locking capabilities to SAM stimuli.. 144

5.12 Bicuculline and strychnine cause a reduction in vector strengths.................... 145

\section{Index of Tables}

\section{Chapter 2}

2.1 Classification of glycine and GABA immunoreactivities in SOC neurons..............45

2.2 Unbiased estimates if Nissl stained and GAD IR neurons in the rat SOC.............. 46

2.3 Morphometric analysis of GAD labeled SPON neurons......................... 47

\section{Chapter 3}

3.1 Unbiased stereological estimates of the number of neurons $\left(\mathrm{N}_{\mathrm{est}}\right)$ found in subcortical auditory structures in the rat..................................................... 74

3.2 Comparison of present results with previously published estimates of the number of neurons in the central auditory structures of the rat.............................. 75 


\section{List of Abbreviations}

$\begin{array}{ll}\text { 7n } & \text { facial nerve } \\ \text { AVCN } & \text { anteroventral cochlear nucleus } \\ \text { BBN } & \text { broad band noise } \\ \text { BF } & \text { best frequency } \\ \text { BIC } & \text { bicuculline } \\ \text { bic } & \text { brachium of the inferior colliculus } \\ \text { CA3 } & \text { field CA3 of hippocampus } \\ \text { cblm } & \text { cerebellum } \\ \text { CN } & \text { cochlear nuclei } \\ \text { CP } & \text { commissure of Probst } \\ \text { crtx } & \text { cortex } \\ \text { DCN } & \text { dorsal cochlear nucleus } \\ \text { DMPO } & \text { dorsomedial periolivary nucleus } \\ \text { DNLL } & \text { dorsal nucleus of the lateral lemniscus } \\ \text { FN } & \text { facial nucleus } \\ \text { GABA } & \text { gamma-amino butyric acid } \\ \text { GAD } & \text { glutamic acid decarboxylase } \\ \text { HCG } & \text { horizontal cell group } \\ \text { IC } & \text { inferior colliculus } \\ \text { IC-1 } & \text { lateral inferior colliculus } \\ \text { IC-m } & \text { medial inferior colliculus } \\ \text { IR } & \text { immunoreactive } \\ \text { LGBd } & \text { dorsal lateral geniculate body } \\ \text { LNTB } & \text { lateral nucleus of the trapezoid body } \\ \text { LSO } & \text { lateral superior olive } \\ \text { mcp } & \text { middle cerebellar peduncle } \\ \text { MGBd } & \text { dorsal division of the medial geniculate body } \\ \text { MGBm } & \text { medial division of the medial geniculate body } \\ \text { MGBv } & \text { ventral division of the medial geniculate body } \\ \text { MNTB } & \text { medial nucleus of the trapezoid body } \\ \text { ms } & \text { milliseconds } \\ \text { MSO } & \text { medial superior olive } \\ \text { PAG } & \text { periaqueductal gray } \\ \text { PVCN } & \text { posteroventral cochlear nucleus } \\ \text { PSTH } & \text { peristimulus time histogram } \\ \text { RM } & \text { response map } \\ \text { SAM } & \text { sinusoidally amplitude modulated } \\ \text { sgl } & \text { superficial granule layer } \\ \text { SOC } & \text { superior olivary complex } \\ \text { SPON } & \text { superior paraolivary nucleus } \\ \text { STRYCH } & \text { strychnine } \\ \text { TCC } & \text { tecto-commissural column } \\ \text { tb } & \text { trapezoid body } \\ \text { Th } & \text { threshold } \\ & \end{array}$


VNLL ventral nucleus of the lateral lemniscus

VNTB ventral nucleus of the trapezoid body

VPO ventral periolivary region 
Chapter One

\author{
Introduction
}




\section{LITERATURE REVIEW}

\section{Introduction}

The mammalian auditory system consists of a peripherally located sound collecting device (the pinna), a sound conducting system (the tympanic membrane and middle ear ossicles), mechano-receptive hair cells in the cochlea, the auditory nerve and a complex neural circuitry within the central nervous system (Kingsley, 1996). The primary functions of the auditory system are to detect and convert sound pressure waves collected by the external ear into action potentials and through an elaborate neural circuitry to characterize the sound's frequency, intensity, envelope (fluctuations in amplitude) and location in the environment. The auditory system is organized by sound frequency place code, or tonotopy, which is first established in the cochlea along the basilar membrane. This organization is maintained throughout the auditory pathways such that most (but not all) auditory nuclei have a tonotopic axis where frequency is represented along a gradient.

Inhibitory neurotransmission, mediated by gamma-amino butyric acid (GABA) and glycine, plays an important role in many auditory processes including sharpening receptive fields, timing of action potentials, sound localization, and sensitivity to sound duration (Schwartz, 1992; Casseday et al., 1994, Fuzessery and Hall, 1996; Park and Pollak, 1993, LeBeau et al., 1996; Yang and Pollak, 1997). Inhibitory neurons are numerous in the mammalian central auditory pathways and are present at each level of the system (Mugnaini and Oertel, 1985; Wenthold et al., 1987; Helfert et al., 1989; Ostapoff et al., 1990).

\section{Superior Olivary Complex}

The superior olivary complex (SOC) is the first level of the mammalian auditory pathway that receives a major convergence of information from both ears onto individual neurons via projections from the cochlear nuclei (Stotler, 1953; Harrison and Warr, 1962; Goldberg and Brown, 1969; Guinan et al., 1972; Warr, 1972). The convergence of information onto SOC neurons is utilized by the nervous system to compare and extract information, such as differences in arrival time and intensity of sounds at the two ears, used to localize sounds in the 
environment. Aside from sound localization, the SOC also provides a descending, modulatory projection to the cochlear nuclei and cochlea.

The SOC consists of up to thirteen distinct nuclei situated in the caudal pons of the brainstem. Three of these cell groups, the so-called principal nuclei, the medial nucleus of the trapezoid body (MNTB), the lateral superior olive (LSO) and the medial superior olive (MSO) (Ramón y Cajal, 1909; Morest, 1968) are conspicuous across mammalian species and as a result, have been studied in detail. The MSO and LSO receive and compare inputs from both ears and have identified roles in sound localization (Stotler, 1953; Harrison and Irving, 1966; Goldberg and Brown, 1968; Glendenning et al., 1985; Friauf and Ostwald, 1988; Brugge, 1991). The MSO and LSO are the main excitatory outflow of the SOC, and both nuclei project to the inferior colliculus (IC; Kumoi et al., 1993; Beyerl, 1978; Coleman and Clerici, 1987; Adams, 1979; Henkel and Spangler, 1983; Kelly et al., 1998). However, the rodent LSO also contains a small population of glycinergic neurons and GABAergic neurons that project to the IC (Saint Marie and Baker, 1990; González-Hernández, 1996). The MNTB is a monaural cell group that receives its main excitatory input from the contralateral cochlear nucleus via the Calyx of Held. The principal neurons of the MNTB use glycine as a neurotransmitter and are therefore inhibitory (Peyert et al., 1987; Wenthold et al., 1987; Aoki et al., 1988; Helfert et al., 1989; Bledsoe et al., 1990). In rodents, the MNTB projects to the LSO, periolivary cell groups and lemniscal nuclei (Banks and Smith, 1992; Sommer et al., 1993). The inhibitory projection to the LSO from the MNTB plays an important role in coding the sound intensity differences between the two ears which is utilized to localize high frequency sounds (Boudreau and Tsuchitani, 1968; Tsuchitani. 1988a,b; Tollin and Yin, 2002a,b).

Scattered throughout the mammalian SOC are up to ten additional nuclei whose neurochemical phenotypes, afferent and efferent connections and physiological response properties are poorly understood and whose contributions to hearing are unknown (Schofield and Cant, 1991; Helfert and Aschoff, 1997). These so-called periolivary nuclei exhibit considerable interspecies variation but tract tracing and morphological studies provide evidence that each periolivary cell group may form a distinct neuronal circuit with a potentially unique role in hearing (Schofield and Cant, 1991; 1992). 


\section{Superior Paraolivary Nucleus}

The superior paraolivary nucleus (SPON) is located within the SOC, dorso-lateral to the MNTB and dorso-medial to the MSO. The size of the SPON varies considerably between species but in rodents, many chiroptera (bats) and some lagomorphs (rabbits) the SPON is rather large compared to other SOC nuclei. It has been suggested that the relative size of the SPON varies inversely with that of the MSO (Kuwabara and Zook, 1993; 1999). The MSO, in many mammals, is associated with low frequency sound localization and is well developed in species with good low frequency hearing. For example, cats can hear frequencies as low as $45 \mathrm{~Hz}$ and have a large, well developed MSO, conversely rats have an almost rudimentary MSO and can hear frequencies only as low as $1000 \mathrm{~Hz}$. This suggests that in a general sense, the SPON will be more prominent in animals with diminished low frequency hearing and a small MSO. In carnivores, the cell group in the region of the SPON is reduced in size and neuronal density and has been termed the dorsomedial periolivary nucleus (DMPO). However, some authors consider the DMPO and SPON homologous structures (Nordeen et al., 1983). Despite some general observations, anatomical and physiological features of the SPON have not been systematically studied in any species. Consequentially, the background information available at this time is compiled from different species.

\section{SPON: Intrinsic Organization}

The morphology of SPON neurons has been examined in Golgi impregnated material in mouse, guinea pig, and rat (Ollo and Schwartz, 1979; Schofield and Cant, 1991; Berrebi and Saldaña, 1997). Mouse SPON neurons have been described as multipolar with long dendrites and appeared morphologically similar in coronal, sagittal and horizontal planes of section, indicating a random dendritic geometry (Ollo and Schwartz, 1979). The guinea pig SPON contains a morphologically heterogeneous neuronal population, dominated by elongated neurons, whose dendritic trees tend to remain within the boundaries of the cell group, and large multipolar neurons (Schofield and Cant, 1991). A detailed morphological examination of SPON cell bodies and dendrites in the rat indicates that this cell group contains a homogenous population of 
multipolar neurons (Berrebi and Saldaña, 1997). In some cases the dendritic trees of ventrally located neurons extend a considerable distance and enter the subjacent ventral nucleus of the trapezoid body (Berrebi and Saldaña, 1997). Both the cell bodies and dendritic trees of rat SPON neurons are flattened parasagittally, resulting in a medial to lateral laminar organization of the nucleus (Saldaña and Berrebi, 2000).

Based on the available data from other species, the SPON appears to contain a heterogeneous population of neurons, the majority of which are multipolar with randomly oriented dendrites. However, the rat SPON contains a homogenous population of multipolar neurons with dendrites that extend for some distance, often leaving the nucleus and entering other nuclei. The dendritic trees of rat SPON neurons are flattened parasagittally, giving the nucleus a laminar organization, a feature that appears necessary for maintaining the tonotopic organization present throughout the auditory pathways.

\section{SPON: Neurochemistry}

Immunocytochemical surveys of the guinea pig SOC have revealed a GABAergic cell population and a distinct glycinergic population in the SPON (Helfert et al., 1989; Benson and Potasher, 1990; Ostapoff et al., 1990; Saint Marie and Baker, 1990). Immunocytochemical studies using antiserum against glutamic acid decarboxylase (GAD, the synthetic enzyme for GABA) in rat and gerbil indicate that the SPON contains a population of GAD immunoreactive, and therefore GABAergic neurons (Oertel et al., 1981; Mugnaini and Oertel, 1985; Moore and Moore, 1987; Roberts and Ribak, 1987; Gonzalez-Hernandez et al., 1996). In some species (cat, gerbil, guinea pig and the Mexican free tailed bat) the SPON/DMPO contains cholinergic neurons that presumably project to the cochlear nucleus and cochlea (Osen and Roth, 1969; Warr, 1975; Nordeen et al., 1983; Thompson and Thompson, 1991a; Grothe et al., 1994). However, cholinergic neurons have not been localized to the rat or mouse SPON (Brown and Howlett 1972; Osen et al., 1984; Vetter et al., 1991; Yao and Godfrey, 1997). Additionally, a single, unconfirmed report of the rat SOC indicates a population of aspartate immunoreactive neurons within the SPON, which presumably use glutamate and/or aspartate as neurotransmitter and are therefore excitatory (Kumoi et al., 1993). 
Thus, in many species the SPON appears to contain GABAergic, glycinergic, cholinergc and possibly excitatory neurons. The rat SPON likely contains only GABAergic and glycinergic neurons. However, the distribution of inhibitory neurotransmitters and proportion of neurons utilizing each neurotransmitter in the SPON has not been studied in detail and therefore remains unclear.

SPON: Afferents

Most of what has been revealed regarding the sources of input to the SPON has been uncovered with tract tracing experiments in mouse, rat and guinea pig. The main excitatory input to the SPON arises from the contralateral ventral cochlear nucleus (VCN; Friauf and Ostwald, 1988; Kuwabara et al., 1991; Thompson and Thompson, 1991a,b; Schofield, 1995). This projection arises from the octopus and multipolar cells (Friauf and Ostwald, 1988; Schofield, 1995). Rat SPON neurons also receive a projection from the ipsilateral VCN (also arising from octopus and multipolar cells), however this projection is much smaller than the contralateral projection (Friauf and Ostwald, 1988; Schofield, 1995). Tract tracing evidence suggests that the projection from the $\mathrm{CN}$ maintains topography and anterograde labeling of $\mathrm{CN}$ axons indicates that this projection may be tonotopically organized (Friauf and Ostwald, 1988; Thompson and Thompson, 1991; Schofield, 1995). Additionally, recent tract tracing data provide evidence that the multipolar cell projection to the SPON is sparse but also maintains tonotopy (Doucet and Ryugo, 2002). Some SPON/DMPO neurons in cat, but not rodents, receive input from collaterals of calyciferous axons arising from globular bushy cells in the contralateral VCN (Morest, 1968; Friauf and Ostwald, 1988; Kuwabara and Zook, 1991; Schofield, 1991; Smith et al., 1991). The projections from octopus, bushy and multipolar cells are presumed to be excitatory; however, glycinergic multipolar cells have been described in the VCN and so some proportion of the input arising from the cochlear nuclei may be inhibitory (Wenthold et al., 1987; Kolston, 1992). Neurons of the ipsilateral MNTB, carrying information from the contralateral cochlear nucleus, send a strong, topographic projection to the SPON (Morest, 1968; Browner and Webster, 1975; Spangler et al., 1985; Kuwabara and Zook, 1991; Banks and Smith, 1992; Kuwabara and Zook, 1992; Sommer et al., 1993). The MNTB 
projection to the SPON is glycinergic and therefore inhibitory (Helfert et al., 1989; Bledsoe et al., 1990). MNTB axons ramify within a number of nuclei, namely the SPON, LSO and ventral nucleus of the lateral lemniscus in rat, however terminal arbors of MNTB axons in the SPON are as extensive if not more extensive than those found in the LSO (Sommer et al., 1993). This provides evidence that the primary target of the rat MNTB is the SPON. The gerbil SPON reportedly receives a projection from the ipsilateral MSO (Kuwabara and Zook, 1993; 1999), a projection reportedly absent in rat (Colombo et al., 1996). A descending projection arising from the inferior colliculus to the SPON has been described (Van Noort, 1969; Thompson and Thompson, 1989; Spangler and Warr, 1991). This finding, however, may be a matter of interpretation as other authors have failed to find an IC-SPON projection in rat (Faye-Lund, 1986; Caicedo and Herbert, 1993; Colombo et al., 1996). Recently, a group of cells situated in the commissure of the inferior and superior colliculi, termed the tecto-commissural column (TCC), has been shown to send a strong projection to the SPON in rats and may be at least partially GABAergic (Mugnaini and Oertel, 1985; Viñuela and Saldaña, 2001). The proximity of the TCC to the IC makes it possible to label TCC neurons (and axons) from a large tract tracer injection made into the IC. Thus, it is likely that the descending projection described from the IC to the SPON included labeled axons from TCC, not from neurons within the inferior colliculus itself. A descending projection to the SPON from the subparafasicular thalamic nucleus (SPF), a cell group that receives projections from numerous cortical areas, has also been described in rat (Yasui et al., 1992). Axons from the SPF target, among other midbrain and hindbrain structures, a number of auditory structures but send the strongest projection to the SPON. The descending projection from SPF is likely inhibitory as GABAergic SPF neurons innervate the IC (Moriizumi and Hattori, 1992; Takada, 1993). The TCC and SPF receive input related to other modalities (somatosensory, visual, motor) and may provide an integrated mulitmodal input to the SPON. Lastly, serotonin, enkephalin and substance $\mathrm{P}$ immunoreactive punctate profiles reportedly contact SPON neurons (Thompson, 1994; Reuss et al., 1999). These serotonin and peptidergic inputs likely arise from neurons outside the auditory pathways and suggest that the neuronal activity of the SPON may be modulated by non-auditory centers

The distribution of neurotransmitter receptors has been surveyed in the SOC using immunocytochemistry and/or in situ hybridization. In line with the known excitatory inputs to 
this cell group, SPON neurons contain NMDA and AMPA glutamate receptors (Sato et al., 1999; Caicedo et al., 1999). SPON neurons express receptors for serotonin (Thompson et al., 1994), GABA $\left(\mathrm{GABA}_{\mathrm{A}}\right)$ and glycine (Friauf et al., 1997; 1998) in line with the known inhibitory inputs. Interestingly, SPON neurons contain the highest level of the alpha 1 glycine receptor subunit out of all brainstem nuclei, indicating a dense glycinergic innervation (Friauf et al., 1997). SPON neurons contain the enzyme guanylate cyclase, which serves as a target for nitric oxide (Bredt and Snyder, 1992; Fessenden et al., 1999). MNTB neurons are the only neurons in the rat SOC to contain neuronal nitric oxide synthase and SPON neurons reportedly contain the highest levels of guanylate cyclase in the SOC. Nitric oxide reportedly inhibits NMDA receptor activity and is involved in long term depression (Shibuki and Okade, 1991; Manzoni and Bockaert, 1993). Thus, the inhibitory input arising from the MNTB may further attenuate excitatory responses in SPON neurons through release of nitric oxide.

Deposits of the bi-directional (transported both anterograde and retrograde) tract tracer biotinylated dextran amine (BDA) in the IC results in retrogradely labelled SPON neurons ipsilateral to the injection site. BDA-labeled punctate profiles, which presumably represent axon terminals, are found apposed to BDA labeled SPON dendrites. The source(s) of these terminals are difficult to verify; however, it was hypothesized that they arise, in part, from SPON neurons (Saldaña and Berrebi, 2000). Thus, another potential source of input to the SPON is other SPON neurons.

In summary, SPON neurons receive a breif onset excitation from octopus cells and a sustained excitatory input from multipolar cells in the contralateral cochlear nucleus. A sustained glycinergic input arises from the MNTB, driven by the globular bushy cells in the contralateral cochlear nucleus. GABAergic inputs may arise from the TCC, SPF and other SPON neurons and the spike pattens of these inputs is unknown. Taken together, this suggests that the rat SPON receives and integrates multiple excitatory and inhibitory inputs that arises primarily from the contralateral ear.

SPON: Efferents 
The projections of the SPON have been studied mainly by way of retrograde tract tracing experiments from potential targets. However, anterograde tract tracing has not been utilized to examine the projections of SPON axons. As a result, there may be other potential targets of SPON axons that have yet to be uncovered. It is widely accepted that the SPON projects to the ipsilateral inferior colliculus (Beyerl, 1978; Zook and Casseday, 1982; Adams, 1983; Moore, 1988; Coleman and Clerici, 1987; Nordeen et al., 1983; Saint Marie and Baker, 1990; Schofield, 1991). The rat SPON projects primarily to the ipsilateral IC while the guinea pig SPON sends a significant projection to the contralateral IC (Schofield, 1991; Saldana and Berrebi, 2000). Placement of a retrograde tracer into the rat IC labels nearly all neurons contained within the SPON ipsilateral to the injection (Saldaña and Berrebi, 2000). Vertical columns of SPON neurons have been shown to project to physiologically defined regions of the ipsilateral IC, providing evidence that the SPON-IC projection maintains tonotopic order (Kelly et al., 1998; Saldaña and Berrebi, 2000). It has recently been shown that the guinea pig, gerbil and rat SPON, much like the cochlear nuclei and ventral nucleus of the lateral lemniscus (VNLL), innervates all subdivisions of the IC, suggesting that the SPON may be involved in numerous aspects of IC processing (Fuentes et al., 1999; Cant and Schofield, 1999). A bilateral projection from the SPON to the TCC has recently been described (Vinuela and Saldaña, 2001) and a projection to the dorsal nucleus of the lateral lemniscus (DNLL) has been demonstrated but a projection to the VNLL has not been examined (Labelle and Kelly, 1997). The guinea pig SPON has been shown to send a descending projection to the cochlear nucleus and cochlea (Tokunaga, 1988; Schofield, 1991; Ostapoff et al., 1997). However, retrograde tract tracing from the rat cochlea has yielded mixed results. Recent tract tracing experiments provide evidence indicating that the rat SPON projects to the cochlea (Riemann and Reuss, 1998; Reuss et al., 1999), but numerous authors have failed to localize olivocochlear neurons to the rat SPON (White and Warr, 1983; Osen et al., 1984; Faye-Lund, 1986; Aschoff and Ostwald, 1987; Vetter et al., 1991; 1993; Vetter and Mugnaini, 1992; Horváth et al, 2000). In cat and ferret a projection from the SPON/DMPO to the superior colliculus has been demonstrated (Edwards et al., 1979; King et al., 1998). The TCC is a group of cells that extends from the commissure of the IC to the commissure of the superior colliculus, which in rat spans nearly four millimeters rostrocaudally. A projection from the SPON to the superior colliculus is supported by the recent evidence of a SPON projection to the rostral extension of the TCC within the superior colliculus (Viñuela and Saldaña, 2001). 
The SPON, in some species, contributes to both the ascending and descending auditory pathways through projections to the IC and cochlear nucleus. However, the rat SPON contributes only to the ascending pathway and the main projection targets of the rat SPON are the DNLL, IC and TCC.

SPON: Physiology

To date, very little has been done to systematically characterize rat SPON responses to sound. The available data regarding SPON/DMPO physiology is gathered largely from experiments in cat and gerbil. A small number of SPON/DMPO neurons $(n=20)$ were recorded in the cat SOC (Guinan et al., 1972a,b). Of the twenty units recorded in this study, thirteen (65\%) were monaural. Responses to pure tone stimuli were recorded for seventeen neurons, about half of which responded at the stimulus offset, while the other half responded during the stimulus. It was noted that SPON/DMPO neurons had broad receptive fields. Most VCN neurons (multipolar) have narrow receptive fields, although octopus cells have wide receptive fields. Thus, wide receptive fields in SPON/DMPO neurons could result from octopus cell input or partially overlapping, convergent input from multipolar cells. In part of a larger work focusing on interaural timing differences in gerbil MSO, recordings were made from a few SPON neurons (Spitzer and Semple, 1995). Onset and offset responses to pure tone stimuli were observed and some neurons phase locked to low frequency tones and were sensitive to interaural phase differences, implicating a potential role in sound localization. The first in-depth studies of SPON physiology were performed in gerbils (Behrend et al., 2002; Dehmel et al., 2002) and these two studies contradict each other in many aspects. For example, Dehmel found that nearly $70 \%$ of gerbil SPON neurons responded at the offset of pure tone stimuli, while Behrend found that only $5 \%$ of their neuronal population responded at the stimulus offset. Further, Dehmel found that $9 \%$ of gerbil SPON neurons were excited by both ears, while Behrend found that $35 \%$ of their population of gerbil SPON neurons were driven binaurally. Because of the conflicting results, the work on the gerbil SPON is difficult to interpret and has done very little to clarify the functional role of the SPON in hearing. The discrepancies between these two gerbil studies are likely due to problems with localization of recording sites, such that 
neurons recorded outside the SPON may have been included in their analyses. Unfortunately, it is difficult to say which data actually represents the gerbil SPON. Interestingly, best frequencies (BFs) of gerbil SPON neurons spanned the hearing range of this animal $(700 \mathrm{~Hz}$ to $37 \mathrm{kHz})$, however the vast majority of neurons had BFs below 6kHz (Behrend et al., 2002) and no clear tonotopic organization of BFs was observed within the SPON (Dehmel et al., 2002). Sinusoidally amplitude modulated pure tone stimuli (SAM) were used to assess phase locking capabilities and sensitivity to the envelopes of complex sounds and many gerbil SPON neurons followed SAM stimuli with high fidelity, suggesting a possible role in the processing of complex auditory signals (Behrend et al., 2002). Recordings from a small number of rat SPON neurons have been reported (Finlayson and Adams, 1997). In contrast to the gerbil and cat physiology and the rat connectivity data, they indicate that SPON neurons are largely binaural, with $79 \%$ of neurons receiving matched best frequency $(\mathrm{BF})$ excitation from both cochlear nuclei.

Based on the available literature, is it difficult to draw any firm conclusions regarding how SPON neurons respond to sound. Despite the report of Finlayson and Adams, one might expect that most rat SPON neurons would be responsive only to contralateral stimulation based on the connectivity data. Further, it is difficult to predict how SPON neurons will integrate onset and sustained excitation from octopus and multipolar cells in the cochlear nucleus, a sustained glycinergic input arising from the MNTB and GABAergic input from multiple sources.

\section{Input to the Inferior Colliculus}

The IC constitutes a considerable portion of the mammalian midbrain and is considered a site of auditory integration, receiving input from virtually all ascending and descending auditory projections. The IC is typically divided into three main divisions, the central nucleus (CNIC), and the dorsal and external cortices (DCIC and ECIC), all of which receive projections from the SPON (Cant and Schofield, 1999; Fuentes et al, 1999). Recently effort has been made to simplify the divisions of the IC into medial (including the central nucleus and the dorsal cortex) and lateral IC (similar to the external cortex) based on tract tracing data. The rationale behind this nomenclature is based on the finding that the inputs and outputs of the central nucleus and dorsal cortex are nearly identical and distinct from those of the external cortex (Saldaña and 
Merchán, 1992).

Inputs to the IC are both excitatory and inhibitory. Inhibition, originating from lower centers, has been shown to be essential for many aspects of collicular physiology. GABAergic and glycinergic inhibition have been shown to sharpen frequency tuning of individual cells and to modify the timing of action potentials in responses to pure tone stimuli (Yang et al., 1992; Lu and Jen, 2001). In the IC, a population of neurons is excited by stimulation of the ipsilateral ear and inhibited by stimulation of the contralateral ear; these cells are termed EI neurons. Similar responses are encountered in the LSO (Guinan et al., 1972b). Extracellular recordings from the IC provide evidence that indicates EI responses are not simply inherited from the LSO but created de novo (Burger and Pollak, 2001). EI neurons are thought to be involved in sound localization and both GABA and glycine contribute to the contralaterally evoked inhibition, which shapes sensitivity to interaural intensity differences (Klug et al., 1995; Park and Pollak, 1993). Recently, GABAergic inhibition has been implicated in creating sensitivity to motion cues, specifically interaural phase modulations (McAlpine and Palmer, 2002). One strategy that the auditory system uses to localize sounds is comparing timing differences between the two ears. Thus, the latency of a cell's response can be crucial to sound localization, and inhibition has been shown to significantly lengthen first spike latencies in IC neurons, thereby delaying the neuron's excitatory response (Park and Pollak, 1993). The precedence effect, a phenomenon where priority is given to a leading sound by suppressing the trailing sound, aids in localizing sound in reverberant surroundings (Hartmann, 1983). It has been hypothesized that a longlasting inhibition, evoked by the leading stimulus is essential for the precedence effect (Litovsky and Yin, 1998). Inhibition has been shown to affect responses to complex stimuli, such as amplitude and frequency modulations (Koch and Grothe, 1998). Amplitude modulations are rapid fluctuations in the intensity of a stimulus and in the laboratory are usually sinusoidally modulated; frequency modulations are changes in the frequency of the tone over time. Blocking both GABA and glycine expands the upper range of amplitude modulation that neurons can follow but decreases the precision of synchronization. Neurons in the bat IC have been shown to display combination sensitivity, such that neurons respond preferentially to combinations of spectral elements (O'Neill and Suga, 1982). Recently it has been demonstrated that glycine is essential for the facilitation observed in combination-sensitive neurons (Wenstrup and Leroy, 
2001). One of the characteristics of biologically relevant sounds, such as vocalizations or echolocation calls, is duration, and neurons tuned to specific durations (or range of durations) have been described in the IC (Casseday et al., 1994; Ehrlich et al., 1997; Brand et al., 2000). Integration of both excitatory and inhibitory inputs, verified by in vivo intracellular recordings, are essential to the formation of duration tuned neurons (Casseday et al., 1994). Additionally, duration tuning is eliminated by application of bicuculline and strychnine, antagonists of $\mathrm{GABA}_{\mathrm{A}}$ and glycine receptors (Casseday et al., 2000).

\section{RATIONALE}

The inhibition reaching the IC from lower auditory centers is complex and plays an essential role in numerous aspects of auditory processing in the IC. Thus, the inhibition arising from the SPON has a potentially important role in hearing and justifies further study of this cell group. Understanding the functional role of the SPON will contribute to our understanding of inhibitory mechanisms utilized in auditory processing. A thorough understanding of the excitatory and inhibitory circuits the auditory system utilizes to encode both simple and complex sound is necessary to improve existing prosthetic hearing devices and the quality of life of those requiring such devices. Further, presbycusis or age-related hearing loss has been partially attributed to diminished inhibition in the IC (Caspary et al., 1999) and an understanding of the role of inhibitory circuits involved in collicular processing will be necessary as strategies to combat presbycusis are developed.

Based on the literature, it is clear that the rat SPON relays information arising from the contralateral ear to the ipsilateral IC, and the nature of this projection may be inhibitory. However, it remains difficult to hypothesize a functional role for this cell group. The fact that the SPON relays inhibitory information to the IC implicates this cell group in a wide range of functions. Inhibition arising from the SPON may serve as lateral inhibition to sharpen frequency response areas or may modulate temporal response properties. Additionally, inhibitory innervation of the IC is necessary for sound localization and processing complex sounds. In short, the SPON could be involved in any aspect of IC processing that relies on inhibitory input. Regardless, elucidating the function of the SPON in any species awaits an in depth and 
systematic study of the neurochemical phenotypes and physiology of this cell group.

Therefore, the purpose of this dissertation is to use immunocytochemical, stereological, physiological and pharmacological techniques to characterize rat SPON neurons in the rat with the hope of clarifying the functional roles of this cell group. Understanding the role of the SPON in hearing will not only further our knowledge of how the auditory system works but will provide insight into the functional role of inhibitory input to the inferior colliculus that may be useful in clinical strategies.

\section{OBJECTIVES}

\section{Study I: What neurochemical phenotypes are present in the SPON?}

The literature provides evidence that the SPON contains populations of inhibitory neurons. However, the proportion of SPON neurons that are GABAergic and glycinergic (or both) remains unclear. We will utilize immunocytochemical techniques with antibodies directed against glycine, GABA and glutamic acid decarboxylase (GAD, the synthetic enzyme for GABA) to examine the presence of inhibitory neurotransmitters in SPON neurons and determine the proportion of neurons utilizing each transmitter. Additionally, we will characterize the inhibitory innervation to the SPON by examining the presence of these neurotransmitters in punctate profiles, presumably representing axon terminals. This work will clarify the chemical nature of the SPON output to the inferior colliculus and the degree to which SPON neurons receive GABAergic and glycinergic input.

\section{Study II: What is the relative contribution of the SPON to the rat auditory pathway?}

The relative contribution of the SPON to the auditory pathway is unclear and this cell group is often neglected as a source of inhibition to the inferior colliculus. We will use unbiased stereology, specifically the optical fractionator tool, to estimate the total number of neurons in eighteen subcortical auditory nuclei. The results from this study will elucidate the prominence of the SPON relative to other auditory cell groups and will show how the number of neurons in this 
cell group relates to other sources of inhibitory input to the IC.

\section{Study III: How do SPON neurons respond to sound stimuli?}

How SPON neurons in the rat respond to sound stimuli is largely unknown. We will characterize responses of SPON neurons to simple (pure tones) and complex (noise and amplitude modulated tones) sound stimuli with in vivo extracellular recordings. A detailed analysis of the physiological response properties of SPON neurons to sound will reveal what types of auditory information this cell group is relaying to the IC and will provide considerable insight into the functional role of this nucleus.

\section{Study IV: How does inhibition shape SPON response properties?}

The contribution of GABAergic and glycinergic inhibition to the physiological response properties of SPON neurons remains unclear. In the previous studies, we described the prominence of inhibitory innervation to the SPON and typical responses of these neurons to sound stimuli. We will examine the effects of inhibitory input on the response properties of SPON neurons through extracellular recordings in vivo with multibarrel electrodes to deliver bicuculline (a $\mathrm{GABA}_{\mathrm{A}}$ anatagonist) and strychnine (a glycine receptor antagonist). This experimental design will allow us to dissect the effects of GABA and glycine on response properties of SPON neurons and will provide further insight into the role of inhibitory circuits in the auditory system. 


\section{Chapter Two}

\section{The Superior Paraolivary Nucleus of the rat is a GABAergic nucleus}

This work is published in the Journal of the Association for Research in Otolaryngology 01: 255-269 (2000) 


\begin{abstract}
The presence of the inhibitory neurotransmitters glycine and GABA (gamma aminobutyric acid) and GAD (glutamic acid decarboxylase), the synthesizing enzyme for GABA, was examined by immunocytochemistry in the superior paraolivary nucleus (SPON) of the rat. Only rarely were SPON neurons observed to be glycine-immunoreactive, but the majority were GABAimmunoreactive. Using unbiased stereological counting methods we estimated that this nucleus contains approximately 2500 neurons. Moreover, $90 \%$ of SPON neurons were immunolabeled by antisera directed against either the $65 \mathrm{kD}$ or $67 \mathrm{kD}$ isoforms of $\mathrm{GAD}$, or a third antiserum that recognizes both GAD isoforms. Morphometric analysis of GAD immunolabeled neurons indicated that SPON neurons possess cell bodies and dendritic arbors that are elongated rostrocaudally and relatively flattened parasagittally. Abundant glycine, GABA and GAD immunoreactive punctate profiles presumed to represent, for the most part, presynaptic axon terminals, were observed in apposition to SPON neurons. We conclude that the rat SPON contains a homogeneous population of multipolar GABAergic neurons that receive abundant GABAergic and glycinergic innervation. The vast majority of glycinergic inputs to SPON are presumed to originate in the ipsilateral medial nucleus of the trapezoid body, but the source(s) of its GABAergic innervation remains to be determined.
\end{abstract}




\section{INTRODUCTION}

Inhibition plays a crucial role in the vertebrate nervous system, and glycine and gammaaminobutyric acid (GABA) are the major inhibitory neurotransmitters in the CNS (Nicholls 1994). Although glycine is a common amino acid present in all proteins and cells, GABA is synthesized from the amino acid glutamate by the enzyme glutamic acid decarboxylase (GAD) (Roberts and Frankel 1950; Nicholls 1994). Because GABA is a small, labile molecule that can be difficult to localize with immunocytochemistry, GAD is widely considered a reliable marker for GABAergic neurons (Wu et al. 1973; Oertel et al. 1981). Two isoforms of GAD, named according to their differing molecular weights, have been isolated (Bayon et al. 1977; Spink et al. 1983; Denner and Wu 1985; Legay et al. 1987; Kaufman et al. 1991). Although both GAD isoforms are present in most GABAergic neurons, GAD 65 and GAD 67 (65 and $67 \mathrm{kDa}$ respectively) are transcribed from separate genes and have different affinities for the cofactor pyridoxal 5'-phosphate (Erlander et al. 1991; Martin et al. 1991; Kaufman et al. 1991). Recent immunocytochemical studies suggest that the two isoforms may have different intracellular distributions, with GAD 67 being widely distributed in somata, dendrites and axon terminals while GAD 65 is located predominantly in axon terminals (Esclapez et al. 1994). GAD 67, which is present as the active holoenzyme, is presumably responsible for utilizing glutamate, through the GABA shunt, for metabolic needs or supplying basal levels of GABA for neurotransmission (Denner and Wu 1985; Erlander and Tobin 1991). In contrast, GAD 65 is active only in the presence of cofactor and synthesizes GABA in terminals when there is increased demand (Martin and Rimwall 1993).

The neurotransmitters GABA and glycine are found in abundance in central auditory nuclei, and inhibitory neurotransmission plays a fundamentally important role in the function of the mammalian auditory system (Helfert and Aschoff 1997). Inhibition has been shown to sharpen tuning curves and modulate discharge properties of auditory neurons, and is crucial to the ability to localize high frequency sounds (Finlayson and Caspary 1989; Yang et al. 1992; Caspary et al. 1994; Park and Pollak 1993; Klug et al. 1995; Le Beau et al. 1996; Koch and Grothe 1998). GABAergic and glycinergic neurons are especially prominent in the superior olivary complex (SOC) (Helfert et al. 1989; Spirou and Berrebi 1997; Saint Marie and Baker 1990; Ostapoff et al. 1990; Ostapoff et al. 1997). This auditory brainstem center contains three principal nuclei with 
identified roles in sound localization, the medial nucleus of the trapezoid body (MNTB), the medial superior olive (MSO) and the lateral superior olive (LSO), and several accessory or periolivary cell groups whose roles in audition are poorly understood (Schwartz 1992; Helfert and Aschoff 1997).

The superior paraolivary nucleus (SPON) is a conspicuous periolivary nucleus in the SOC of rodents and guinea pigs. Situated medial to the LSO and dorsolateral to the MNTB, this nucleus receives ascending inputs from the contralateral cochlear nucleus (Friauf and Ostwald 1988; Kuwabara et al. 1991; Thompson and Thompson 1991a,b; Schofield and Cant 1995), as well as a substantial local inhibitory input from the ipsilateral MNTB (Kuwabara and Zook 1991, 1992a,b; Banks and Smith 1992; Sommer et al. 1993), and sends a prominent projection to the ipsilateral inferior colliculus (IC) (Beyerl 1978; Adams 1983; Coleman and Clerici 1987; Moore 1988; Saint Marie and Baker 1990; Gonzalez-Hernandez et al. 1996; Fuentes et al. 1999; Saldaña and Berrebi, 2000). Notably, the SPON of the rat does not receive a descending projection from the IC nor does it project to the cochlear nucleus or cochlea (White and Warr 1983; Osen et al. 1984; Faye-Lund 1986; Aschoff and Ostwald 1987; Vetter et al. 1991, 1993; Vetter and Mugnaini 1992; Caicedo and Herbert 1993).

A number of immunohistochemical and retrograde transport studies have revealed populations of GABA-immunoreactive (IR) and glycine-IR neurons in the guinea pig SPON, suggesting that these two neurochemically defined cell groups have different projection targets (Helfert et al. 1989; Thompson et al. 1985; Saint Marie and Baker 1990; Ostapoff et al. 1985, 1990). Recent tract tracing data in rats, however, demonstrate a homogeneous neuronal population with a purely ipsilateral ascending projection to the IC (Berrebi et al. 1997; Saldaña and Berrebi 2000). Using antibodies directed against GABA or an antiserum that recognizes both isoforms of GAD, other investigators have suggested that the rat SPON contains a substantial proportion of GABAergic neurons (Oertel et al. 1981; Mugnaini and Oertel 1985; Roberts and Ribak 1987; Moore and Moore 1987; Gonzalez-Hernandez et al. 1996). Gly-IR has been described in the rat SOC (Campistron et al. 1986; Aoki et al. 1988; Pourcho et al. 1992), but has not been systematically examined in the SPON or in other periolivary cell groups. 
In order to gain a better understanding of the functional role of SPON, we used immunocytochemical methods to delineate the distribution of GABAergic and glycinergic neurons and axon terminals within the nucleus. We also obtained unbiased stereological estimates of the percentage of SPON neurons that are GAD-IR, and performed a quantitative morphometric assessment of SPON neuronal morphologies. Some of the data contained herein have been presented in abstract form (Kulesza et al. 1998; Kulesza and Berrebi 1999).

\section{MATERIALS AND METHODS}

\section{Animals}

Adult female Sprague-Dawley rats, weighing 230-285g, were deeply anesthetized by an intramuscular injection of a mixture xylazine and ketamine (42mg xylazine and 57mg ketamine per $100 \mathrm{~g}$ body weight) prior to vascular perfusion. A total of 36 animals were used for this study. The West Virginia University Institutional Animal Care and Use Committee approved all animal protocols used for this project.

\section{Post-embedding immunocytochemistry for glycine and GABA}

Twelve animals were perfused through the ascending aorta with a rinse of $\mathrm{Ca}^{++}$free Ringer's variant followed by one liter of fixative composed of $2 \%$ paraformaldehyde and $2.5 \%$ glutaraldehyde in $0.1 \mathrm{M}$ sodium phosphate buffer. Animals were placed on ice and remained undisturbed for 30 minutes before their brains were dissected and immersed in fixative overnight. The brains were sectioned in the coronal plane using a Vibratome, into $100 \mu \mathrm{m}$ thick sections which were postfixed in $1 \%$ osmium tetroxide, stained with $2 \%$ aqueous uranyl acetate, dehydrated, and flat embedded in Epon (Polybed 812, Polysciences). After polymerization, sections containing the SOC were cut into a series of $1 \mu \mathrm{m}$ semithin sections, heat-dried onto glass slides and prepared for immunocytochemistry (Spirou and Berrebi 1997). Briefly, Epon was etched from the sections using sodium ethoxide (absolute ethanol saturated with sodium hydroxide). The tissues were then rehydrated, first in ethanols and then in water. Osmium was subsequently removed with $1 \%$ aqueous sodium periodate. 
Adjacent pairs of $1 \mu \mathrm{m}$ sections were incubated in $5 \%$ normal donkey serum in $0.5 \mathrm{M}$ Trizma base- $\mathrm{HCl}$ (Tris, $\mathrm{pH}$ 7.6) for 1 hour followed by overnight incubation in a primary antiserum as follows: rabbit ant-glycine (Chemicon, Temecula, CA, diluted 1:1200), rabbit antiGABA (gift from Dr. D. Pow, University of Queensland, Brisbane, Australia, diluted 1:8000 or purchased from HTI Bio-Products, Inc., Ramona, CA, now Strategic BioSolutions; diluted 1:1000). Sections were then incubated in biotinylated secondary antiserum and further processed using the $\mathrm{ABC}$ method (Vector, Burlingame, $\mathrm{CA}$ ). The chromogenic reaction took place in $0.05 \%$ diaminobenzidine (DAB) with $0.01 \%$ hydrogen peroxide in $0.5 \mathrm{M}$ Tris.

The specificity of the Chemicon glycine- and Pow GABA- antisera has been previously established in our laboratory with preadsorbtion control experiments using an amino acid inhibitor complex according to Storm-Mathisen and Ottersen (1990) (see figure 1 of Spirou and Berrebi 1997). The affinity purified, anti-GABA serum from HTI Bioproducts was reported to display approximately $2 \%$ cross reactivity with glycine. In the present experiments, we tested the effects of antiserum preadsorption on intensity of immunoreactivity using an optical densitometric procedure (see below).

\section{Optical density}

To classify individual cells according to their glycine and/or GABA immunoreactivities, we performed optical densitometry in adjacent semithin (1 $\mu \mathrm{m}$ thick) sections. A total of six pairs of sections (sections in each pair were within $2-3 \mu \mathrm{m}$ of each other) from the SOC of six animals were used in this analysis. Semithin sections were used because individual neurons could be identified in sequential sections immunostained with either glycine or GABA antiserum. We evaluated every SPON neuron encountered in each section, as well as randomly selected MNTB and MSO cells. The immunostaining intensities of MNTB neurons served as a reference standard for glycine-immunopositivity, and those of MSO cells as an indication of background immunostaining levels.

Images were captured with a SONY video camera mounted on an Olympus BH-2 
microscope (40X objective, final magnification 980X). Average pixel grey values were measured from uniform regions of neuronal cytoplasm. Care was taken so sampling regions within the neuronal cytoplasm did not include the pale staining cell nuclei. The grey value intensity scale was calibrated for each pair of sections so that the darkest staining soma (i.e. a glycine-IR MNTB neuron) was assigned a value of 255 and the lumen of a blood vessel was assigned a value of 0. Data were entered into JMP (SAS Institute Inc., Cary, NC) and Statview (Abacus Concepts, Inc., Berkeley, CA) data analysis software packages for statistical comparisons and graphical output.

In one pair of sections, the glycine antiserum was preadsorped with 5mM GABA and the GABA antiserum was preadsorbed with $10 \mathrm{mM}$ glycine before incubation with the tissue. The optical density data obtained from this pair of sections was statistically indistinguishable from the remaining five pairs of sections which were not incubated in preabsorbed antisera $(\mathrm{t}=1.819$, p>.07 for GABA antiserum; and t=1.056, p>.29 for glycine antiserum). Therefore, the effect of preabsorption will not be considered further. We also compared the immunostaining patterns obtained with the GABA antiserum provided by Dr. Pow with the commercially available (HTI Bioproducts) antiserum. Within the range of dilutions we tested, the latter resulted in somewhat weaker staining intensities throughout the brain, but the pattern of immunostaining obtained within nuclei of the SOC were judged to be qualitatively equivalent. Therefore, the data obtained from both GABA antisera were pooled for all analyses.

\section{GAD immunocytochemistry on frozen sections}

A total of twenty-four animals were perfused through the ascending aorta with a vascular rinse of physiological saline, followed by a fixative composed of $4 \%$ commercial formaldehyde and a zinc salt $(0.5 \%$ zinc dichromate, $\mathrm{pH} 4.0$, or $0.5 \%$ zinc salicylate, $\mathrm{pH} 6.5)$ in $0.9 \%$ saline (Mugnaini and Dahl 1983; Berrebi and Mugnaini 1991). The animals were placed on ice for 30 minutes before brains were dissected and immersed in cryoprotectant (30\% sucrose in saline) for at least 24 hours. Brainstems were sectioned in series order, either coronally or parasagittally, at a thickness of $30 \mu \mathrm{m}$ on a freezing microtome. 
Free-floating sections were rinsed in $0.5 \mathrm{M}$ Tris, blocked in 5\% normal donkey serum and incubated for 48 hours at $4^{\circ} \mathrm{C}$ in primary antiserum with gentle agitation. To optimize the immunostaining, different protocols were used depending on the primary antiserum. GAD-1440 antiserum (Oertel et al. 1981; provided by Judith Harvey-White, NIH, Bethesda, MD and used at a dilution of 1:1200) is a sheep polyclonal antiserum that recognizes both GAD isoforms. GAD-6 (Chang and Gottlieb 1988; purchased from Developmental Studies Hybridoma Bank, University of Iowa and used at a dilution of 1:1000) is a mouse monoclonal antiserum directed against the GAD 65 isoform. Both of these antibodies were revealed using the PAP method (Sternberger 1979), although the best results with the GAD-6 antiserum were obtained with the double PAP method (Ordronneau et al. 1981). K2 antiserum (Kaufman et al. 1991, sold by Chemicon, Temecula, CA and used at a dilution of 1:3000) is a rabbit polyclonal antiserum that primarily recognizes GAD 67 and was revealed according to the $\mathrm{ABC}$ method. The chromogenic reaction for each antiserum took place in $0.05 \% \mathrm{DAB}$ with $0.01 \%$ hydrogen peroxide in $0.5 \mathrm{M}$ Tris. For reference, alternating sections were not immunoreacted, but stained for Nissl substance using cresyl violet according to standard protocols. All sections were mounted onto glass slides from gelatin-alcohol, air-dried and coverslipped using Accumount.

Specificity assays (preabsorption controls and Western blots) using the GAD-6 and K2 antisera were performed previously by Esclapez and co-workers (1994) who indicate that the GAD 67 specific K2 antiserum has a slight cross-reactivity with GAD 65 and that GAD-6 antiserum does not crossreact with GAD 67. The specificity of the GAD-1440 antiserum for GAD has been established (Oertel et al. 1981). This antiserum localizes to both isoforms of the enzyme (Kaufman et al. 1991).

\section{Morphometric Analysis}

Frozen sections immunoreacted with GAD-1440 or GAD-6 antiserum were sampled at $90 \mu \mathrm{m}$ intervals for further analysis. A camera lucida was used to trace all labeled neurons containing nuclei using a 100X oil immersion objective (final magnification of tissue 1100X). Care was taken to estimate the cell body contour so that dendrites extending from the soma were not included. The drawings were scanned into a Power Macintosh computer, and NIH Image 
software (version 1.61, NIH, Bethesda, MD) was used to measure the area, perimeter and major and minor diameters of each cell body. An index of circularity (Yin et al. 1990) was then

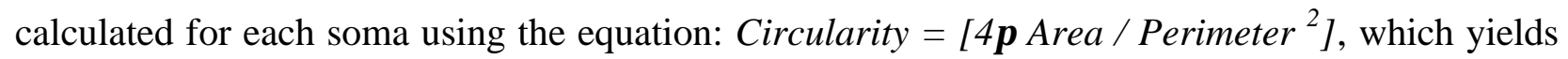
an estimate of cell shape that is independent of size. Using this formula, a perfectly circular cell body profile would be assigned a circularity value of 1.0, with increasingly elliptical profiles resulting in decreasing values. Statistical comparisons were performed using the Statview data analysis software package.

\section{Unbiased Stereology}

We estimated the number of Nïssl stained and GAD-IR neurons in the SPON using methods of unbiased stereology (Gundersen 1988a, b). The Computer Assisted Stereological Toolbox system (C.A.S.T.-Grid, Olympus, Denmark) was used to implement the optical fractionator technique (Gundersen 1986), which incorporates the optical disector tool (Sterio 1984). We randomly selected a slide caudal to the SPON as a starting point and sampled uniformly spaced sections (every fourth or fifth section) through the nucleus using the optical

disector tool. A counting volume (approximately 33,600 $\mathrm{m}^{3}$ ) was defined within the thickness of the tissue section and only cells that were contained and in focus within the appropriate boundaries of the counting frame were marked. Our final estimates were calculated using the equation:

$$
N=\text { [number of cells counted * area fraction * section fraction * disector fraction]. }
$$

\section{Quantitative Analysis of Puncta in Plastic Sections}

The number of perisomatic puncta was quantified at 100X under oil immersion (final magnification 1100X). Measurements of puncta perimeter and long and short axis were made on the computer monitor with the C.A.S.T.-Grid software package using the distance/boundary function. The area of punctate profiles was calculated by modeling them as ellipses using the equation:

$$
\text { Area }_{\text {puncta }}=[0.5 \text { Major Axis } * 0.5 \text { Minor Axis } * \pi] .
$$




\section{RESULTS}

The subset of SOC sections stained with cresyl violet was used to assist in delineating the boundaries of the SPON and other SOC nuclei. A Nïssl stained coronal section of the rat pons at a middle rostro-caudal level through the SOC is shown for reference in Figure 2.1. At this level, all three principal nuclei (LSO, MSO and MNTB) can be identified. Several periolivary cell groups, including the ventral (VNTB) and lateral nuclei of the trapezoid body (LNTB) and the SPON are also present. The SPON is identified as a prominently sized ovoid shaped nucleus interposed between the LSO, MSO and MNTB. In this material, the SPON appears to possess a low density of neurons relative to the MNTB and LSO.

\section{Overview of glycine and GABA immunoreactivities in the SOC}

Postembedding immunocytochemistry for glycine and GABA revealed immunolabeled somata, dendrites, fibers and punctate profiles, presumed to represent for the most part axon terminals, throughout the SOC (Fig. 2.2). Qualitatively, neurons of the MNTB appeared most intensely glycine-IR, and the VNTB contained most of the intensely GABA-IR neurons. Glycine- and GABA-IR cell bodies of varying staining intensities were also found scattered in the LSO and LNTB, as well as other nuclei. Cell bodies of the MSO appeared immunonegative after incubation with either antiserum.

The boundary between the SPON and LSO was clearly identified by an immunonegative fiber bundle coursing between these nuclei. The medial border of the SPON was somewhat more difficult to define precisely. In some sections, we noted a thin wedge of tissue located dorsolateral to the MNTB that contained a small number of neurons and a densely immunoreactive neuropil (Fig. 2.2). The morphological appearance of these cells suggested that they belong to SPON, but we cannot exclude the possibility that displaced neurons from the MNTB or the reticular formation were occasionally included with the limits of SPON. Nonetheless, inclusive of these few peripherally located cell bodies, the SPON contained only rare somata qualitatively judged to be glycine-IR, and such cells were lightly 
immunostained. Most SPON neurons appeared lightly to moderately GABA-IR, while a small percentage were intensely immunolabeled (Figs. 2.2, 2.3).

Glycine-IR and GABA-IR puncta were observed throughout the SOC. Glycine-IR punctate profiles were densely distributed in the SPON, the middle and lateral limbs of the LSO, the LNTB and the VNTB. GABA-IR punctate profiles were also abundant in the SPON, although at a lower density than glycine-IR profiles (Fig. 2.3).

\section{Quantitative analysis of glycine and GABA immunoreactivities}

A quantitative densitometric method was used to classify the glycine- and GABAimmunoreactivities of SOC neurons (Fig. 2.4). The IR intensity values representing MNTB neurons were localized to the upper portion of the glycine-IR intensity scale and the lower portion of the GABA-IR intensity scale, while values of MSO neurons were clustered at the lower extreme of both intensity scales. The IR intensity values associated with SPON neurons were widely dispersed across nearly the entire GABA-IR intensity scale.

The densitometric data are summarized in Table 2.1. For clarity of presentation, the immunostaining intensity scales were subdivided into equal thirds roughly corresponding to 1) immunonegative or light-IR, 2) moderate-IR or 3) dark-IR. Eighty-nine percent of SPON neurons were immunonegative or displayed light glycine-IR intensity, while $11 \%$ displayed moderate glycine-IR intensity. Approximately two-thirds of SPON cells displayed glycine-IR intensities below, and GABA-IR intensities above those representing the most intensely immunostained MSO cells. Only 9 SPON cells $(<6 \%)$ displayed glycine-IR intensity values greater than 100. Nearly half of SPON neurons were immunonegative or displayed light GABAIR, $41 \%$ displayed moderate GABA-IR, and 10\% displayed dark GABA-IR.

We then performed a logistic regression analysis with group membership (nucleus) as the response and with glycine-IR and GABA-IR intensity values as predictors. Glycine-IR and GABA-IR intensities were both significantly associated with group membership $(\mathrm{p}<.001$ level, likelihood ratio chi-square). All MNTB neurons analyzed were assigned to the correct group 
membership, while $72 \%$ of MSO neurons and $91 \%$ of SPON neurons were assigned to the proper group. The remaining MSO and SPON neurons, which could not be reliably categorized to either group, all displayed low values for both glycine-IR and GABA-IR intensities. Taken together, we concluded from these data that the vast majority of SPONneurons in the rat express light to moderate GABA immunoreactivity.

\section{GABA-IR and glycine-IR puncta in the SPON}

We also performed a quantitative comparison of the innervation densities of SPON neurons by glycinergic and GABAergic puncta. To account for the variations in cross-sectional area of SPON neurons and due to the limited thickness of the sections processed for postembedding immunocytochemistry, we computed the innervation density relative to the perimeter of the SPON neurons. Uniformly throughout the nucleus, SPON somata were apposed to an average of $19.42 \pm 0.72$ (S.E.M.) gly-IR puncta per $100 \mu \mathrm{m}$ of somal perimeter. The cross sectional area of gly-IR puncta measured an average of $2.16 \pm 0.13 \mu \mathrm{m}^{2}$. GABA-IR punctate profiles measured an average of $1.57 \pm 0.91 \mu \mathrm{m}^{2}$ and were found in apposition to somata throughout the SPON. However, somata in the ventrolateral SPON were apposed to an average of $15.04 \pm 0.76$ GABA-IR puncta, while neurons in the dorsomedial portion of the nucleus were apposed to an average of $23.37 \pm 0.96$ puncta, per $100 \mu \mathrm{m}$ of somal perimeter. This difference in innervation density was statistically significant by ANOVA $(\mathrm{p}<0.0001)$.

\section{Overview of GAD-IR in the SOC}

GABA is a small, labile molecule that can be difficult to localize with immunocytochemistry and may not accumulate to detectable levels in somata of neurons that utilize GABA as neurotransmitter (Ottersen and Storm-Mathisen 1984). Moreover, optimal conditions of tissue fixation and processing to reveal GABA by immunocytochemistry are most conducive to post-embedding protocols on thin sections, which are not ideally suited to quantitative morphological and stereological analyses. For these reasons, we chose to employ isoform specific antisera directed against GAD, the synthesizing enzyme for GABA and a reliable marker of GABAergic neurons (Wu et al. 1973; Oertel et al. 1981; Oertel 1983). 
GAD immunocytochemistry revealed cell bodies, dendrites and punctate profiles throughout the SOC (Figs. 2.5, 2.6). The LSO contained a sub-population of IR neurons situated among immunonegative neurons, and abundant GAD-IR punctate profiles. More LSO somata were immunolabeled by GAD-1440 (cross-reacts with both GAD isoforms) and K2 antisera (specific for GAD-67; Figs. 2.5 and 2.6B) than with GAD-6 antiserum (recognizes the $65 \mathrm{kD}$ isoform; Fig. 2.6A). The MSO and MNTB contained no GAD-IR cells, but neurons in both nuclei were surrounded by numerous GAD-IR puncta. Among the periolivary nuclei, the VNTB contained the most intensely labeled neurons and dense punctate labeling. The LNTB also contained both GAD immunopositive and immunonegative somata as well as GAD-IR punctate profiles. The SPON contained many labeled neurons and a moderate density of GADIR puncta. Overall, the labeling of punctate profiles in the SOC was more intense with the GAD 65 specific GAD-6 antiserum.

\section{GAD-IR somata in the SPON}

Regardless of the antisera used, immunolabeling of SPON neurons appeared as a dark reaction product that filled the cytoplasm and often extended into proximal dendrites. GAD-IR neurons appeared to be evenly distributed throughout SPON and it was our impression that the vast majority of SPON neurons were GAD-IR. Unbiased stereology performed in five animals indicated that the rat SPON contains approximately 2,555 neurons (revealed by cresyl violet counterstaining) and 2,313 GAD-IR neurons (Table 2.2). Thus, roughly $90 \%$ of SPON neurons express GAD. The $65 \mathrm{kD}$ and $67 \mathrm{kD}$ isoforms of the enzyme were present in equal numbers of neurons (2,314 and 2,346 neurons, respectively), indicating a great degree of co-expression.

\section{Neuronal Morphology}

SPON neurons exhibited some variability in size and shape (Figs. 2.5, 2.6). In the coronal plane of section, the majority of GAD-IR SPON neurons were either bipolar or oval with a vertical orientation, while a small fraction appeared to have a multipolar morphology. When viewed in the parasagittal plane, SPON neurons typically displayed a more 
homogeneous multipolar appearance with multiple dendrites extending from the soma in various directions (Fig. 2.7). Parasagittally sectioned profiles of SPON somata (traced separately from their dendrites) were significantly larger and less circular than coronally sectioned profiles (Table 2.3). These morphometric data, taken together, indicate that typical GAD-IR SPON neurons are large $\left(335.9 \pm 10.64 \mu \mathrm{m}^{2}\right.$ average cross sectional area in the parasagittal plane), multipolar cells whose somata and dendritic trees are flattened parasagittally.

In both planes of section, GAD-IR dendrites were seen extending between the SPON and the neuropil of the nearby VNTB (Figs. 2.6 and 2.7). In coronal sections, these dendrites appeared to form a single, narrow bundle. In parasagittal sections it was possible to view a considerable portion of the rostro-caudal extent of the SPON, and GAD-IR dendrites appeared as several small bundles separated by distinct immunonegative fascicles of trapezoid body fibers (Fig. 2.7). In some cases it was evident that the GAD-IR dendrites belonged to SPON neurons (Fig. 2.7B).

\section{GAD-IR puncta density in the SPON}

Each of the three antisera used revealed myriad GAD-IR punctate profiles in SPON, which we interpret as largely representing axon terminals. These puncta were apposed to GAD-IR somata throughout the SPON, forming characteristic perisomatic and peridendritic arrays (Fig. 2.8). Some of the puncta were connected by delicate fibers suggesting that they were en passant boutons. GAD-IR puncta were uniform in cross-sectional area throughout the nucleus, measuring an average of $1.58 \pm 0.54 \mu \mathrm{m}^{2}$. 


\section{DISCUSSION}

\section{Constituent neurons of the SPON}

Previous studies of the rat SOC utilizing antisera to GABA or GAD have revealed a GABAergic population of neurons within the SPON (Mugnaini and Oertel 1985; Li et al. 1995; Gonazalez-Hernandez et al. 1996). Mugnaini and Oertel (1985) did not focus on the SPON per se but, using the same non isoform-specific GAD-1440 antiserum used in this study, estimated that between 50 and $90 \%$ of the neurons in the rat SPON were GAD immunoreactive. It has also been reported that aspartate-IR neurons dominate in the rat SPON (Kumoi et al. 1993), but this isolated finding has not been confirmed. In contrast, immunocytochemical studies of guinea pig SPON have described distinct GABAergic and glycinergic neuronal populations (Helfert et al. 1989; Saint Marie and Baker 1990; Ostapoff et al. 1990; Ostapoff et al. 1997). These findings, considered in combination with tract tracing studies in guinea pig, led Schofield (1991) to suggest that somal morphology and neurochemical phenotype are correlated with efferent projection target of SPON neurons. Our data demonstrate remarkable homogeneity of the SPON of the rat, in that the vast majority of rat SPON neurons are immunoreactive for GAD. Moreover, it has been reported recently that virtually all SPON neurons in rat project to the ipsilateral central nucleus of the IC (Saldaña and Berrebi 2000). Taken together, these studies suggest that the rat SPON represents a relatively simply organized nucleus with virtually all of its cells providing a purely GABAergic innervation of the ipsilateral IC. For these reasons, we propose that the rat is an ideal species in which to study the physiology and pharmacology of GABAergic projections.

\section{Co-localization of GAD isoforms in SPON neurons}

The fact that both the 65 and $67 \mathrm{kD}$ isoforms of GAD colocalize in SPON somata is noteworthy. Esclapez and coworkers (1994) reported that in many neurons the two GAD isoforms occupy different intracellular compartments, with GAD 65 being highly concentrated in terminals and GAD 67 distributed in both somata and terminals. However, there is molecular evidence that GAD 65 and 67 can form heterodimers (Sheikh and Martin 1996), and biochemical 
data suggest that GAD 67 is targeted to the perikaryal Golgi membrane via an interaction with GAD 65 (Dirkx et al. 1995). The membrane-bound GAD 65-67 heterodimer is then presumably shipped from the Golgi apparatus to nerve terminals, possibly resulting in the cell body and axon terminal colocalization of GAD isoforms in our study. The production and possible dimerization of GAD 65 in somata may explain our localization of this isoform in SPON perikarya. The precise role of these isoforms in GABAergic neurotransmission and cellular metabolism is not yet understood. Based on counts of GAD-IR neurons we obtained by unbiased stereology, we report that both isoforms were present in the vast majority of SPON neurons. Even though it is difficult to quantify immunolabeling intensity in our material, the intense labeling found with both the GAD-6 and K2 antisera is suggestive that both isoforms are located in somata at relatively high levels. GAD 65 is inactive in the absence of cofactor (pyridoxal 5' phosphate; PLP) and cannot synthesize GABA in cell somata since the cofactor is specifically localized to nerve terminals (Nicholls 1994). Therefore, if GAD 67 is responsible for metabolic processes, such as the GABA shunt which moves glutamate into the Krebs cycle (Baxter 1970), then pools of GABA made by this enzyme are likely to be quickly converted to another metabolite (e.g. succinic semialdehyde) and not be involved in neurotransmission. The inactivity of GAD 65 and the possible metabolic role of GAD 67 might contribute to the common difficulties in revealing GABAergic cell bodies using antisera directed against GABA (e.g. Gonzalez-Hernandez et al. 1996; see also Ottersen and Storm-Mathisen 1994).

\section{Glycinergic and GABAergic inputs to SPON neurons}

Rat SPON neurons express GABA and glycine receptors on their membranes (Friauf et al. 1997, 1998), and we observed abundant glycine-IR and GABA-IR in the neuropil of the nucleus. Our data and previous reports suggest an extremely dense glycinergic synaptic input to SPON which arises, in large part, from MNTB (Morest 1968; Helfert et al. 1989; Kuwabara and Zook 1991, 1992b; Banks and Smith 1992; Sommer et al. 1993). Our results also coincide with the high concentration of glycine reported in the rat SPON by high performance liquid chromatography (Godfrey et al. 2000).

The prominent punctate labeling revealed by GABA and GAD immunocytochemistry 
indicates that SPON also receives GABAergic synaptic input, but the source or sources of this innervation is not known. One candidate is the nearby VNTB, which contains a population of GABAergic neurons (Mugnaini and Oertel 1985; Moore and Moore 1987). In rats, VNTB and SPON display higher GABA concentrations than other SOC nuclei (Godfrey et al. 2000). However, tract tracing studies in rat have failed to show such a projection (Warr and Beck 1996), and we consider it unlikely that the VNTB represents a significant source of GABAergic puncta in the SPON.

The lemniscal nuclei are also possible sources of GAD-IR puncta in the SPON. Both the dorsal and ventral nuclei of the lateral lemniscus (DNLL and VNLL) contain GABAergic neurons (Thompson et al. 1985; Moore and Moore 1987; Roberts and Ribak 1987; GonzalezHernandez et al. 1996; Riquelme et al. 1998). Tract tracing data provide evidence that the DNLL innervates the SOC in rats, although it is uncertain if the SPON receives any of this input (Bajo et al. 1993). The VNLL in cats has been shown to project to the dorsomedial periolivary nucleus (DMPO) (Whitley and Henkel 1984), the presumed homologue of the SPON of rodents, but we are not aware of reports of a similar projection in the rat.

It is also quite possible, in fact likely, that a proportion of GAD-IR puncta within the SPON arises from branches of SPON axons. Preliminary intracellular labeling experiments from our laboratory indicate that SPON axons collateralize before leaving the nucleus and contact other SPON neurons (Kulesza et al. 2000). Such an arrangement suggests that SPON neurons may provide modulatory feedback to local targets in and around the nucleus. We are particularly interested in this possibility, especially given the recent demonstrations that the SPON of the rat is tonotopically organized (Kelly et al. 1998; Saldaña and Berrebi 2000). Further, more refined tracing studies are necessary to adequately resolve these local collaterals and their potential impact on SPON function.

\section{Morphology of SPON neurons}

Our morphometric study complements previous tract-tracing work showing that the rat SPON contains a population of multipolar neurons that project to the inferior colliculus and 
whose somata and dendritic arbors are elongated rostrocaudally and flattened parasagittally (Saldaña and Berrebi 2000). The present data also corroborate the observation that SPON neurons appear different in size and shape depending on the sectioning plane in which they are viewed. Further, their anisotropic organization coincides with the expected geometric arrangement of cells within a tissue displaying a lateral-to-medial tonotopic order of frequency representation, as demonstrated for the SPON (Kelly et al. 1998; Saldaña and Berrebi 2000), since each neuron is expected to receive synaptic inputs localized in mediolaterally restricted territories.

We noted that some ventrally located SPON neurons possessed dendrites that appeared to enter the VNTB, a feature previously demonstrated in retrogradely labeled SPON neurons (Saldaña and Berrebi, 2000). Neither the frequency of occurrence nor purpose served by this outgrowth of dendritic processes is clear at this time. However, one can envision that selected SPON neurons may be "sampling" some of the synaptic input received by VNTB cells, including an excitatory descending projection originating in the IC which does not innervate the SPON directly (Faye-Lund 1986; Rajan 1990; Vetter et al. 1993).

\section{Functional implications}

The SPON receives presumably excitatory projections from octopus and multipolar cells of the ventral cochlear nucleus (Warr 1966; Friauf and Ostwald 1988; Saldaña et al. 1994; Schofield 1995; Finlayson and Adam 1997). Octopus cells display distinct physiological characteristics, such as broad tuning curves and onset responses at characteristic frequency $(\mathrm{CF})$, and pronounced phase locking at low frequency (Godfrey et al. 1975; Rhode et al. 1983; Rhode and Smith 1986). The excitatory multipolar cells, on the other hand, would be expected to provide a frequency tuned and sustained input to SPON (Smith and Rhode 1989). The SPON also receives excitatory input from collaterals of calyciferous bushy cell axons and collaterals of MSO projections (Morest 1968; Smith et al. 1991; Kuwabara and Zook 1999). The integration of excitatory inputs could create a variety of response patterns in the SPON that are difficult to predict. Onset responses could be broadly (from octopus cells) or narrowly (from multipolar and globular cells) tuned, and sustained responses (from multipolar cells) may be narrowly tuned and 
could reveal monaural or binaural (from MSO cells) interactions. These excitatory influences are presumably balanced in SPON by finely tuned glycine-mediated inhibition arising in the MNTB (Banks and Smith 1992; Sommer et al. 1993; Smith et al. 1998) and also by its own GABAergic collateral innervation.

Despite broad tuning in some of its excitatory input, SPON provides a topographic and presumably tonotopic projection to the inferior colliculus (Kelly et al. 1998; Saldaña and Berrebi 2000). GABAergic and glycinergic inhibition originating from the SOC is reported to sharpen tuning curves of IC neurons to certain types of sound (Yang et al. 1992, Koch and Grothe 1998), influence the temporal firing patterns of IC units (LeBeau et al. 1996), and are involved in tuning for duration of sound stimuli in the IC (Casseday et al. 1994). GABA is also a powerful mediator of interaural intensity disparity sensitivity in the colliculus (Park and Pollak 1993). Thus, the inhibitory GABAergic projections of the SPON may influence midbrain auditory circuitry in a manner that enables distinction of subtle changes in the frequency and/or temporal characteristics of sounds. There are no published studies of the physiological response properties of rat SPON neurons that shed light on their contribution to the above mentioned features of collicular physiology. There is evidence from other species that some DMPO and SPON neurons are broadly tuned (Guinan et al. 1972 [cat]; Spitzer and Semple 1995 [gerbil]; Dehmel et al. 1999 [gerbil]). The placement of SPON in a clearer functional context awaits the identification of the source or sources of its abundant GABAergic synaptic input and the physiological response characteristics of its constituent neurons. 


\section{Acknowledgments}

The authors gratefully acknowledge the excellent technical assistance provided by Mrs. Nancy Irby. We are also indebted to Dr. Gerald Hobbs of the WVU Department of Statistics for expert advice concerning statistical analyses. GAD 1440 antiserum was provided from a source developed at NIH by Drs. Irwin J. Kopin, Wolfgang Oertel, Donald E. Schmechel and Marcel Tappaz. Current provider of this antiserum is Judith Harvey-White, Bldg. 36, Rm. 5W-21, Bethesda,MD 20892-4164, e-mail: jdhw@box-j.nih.gov. Sincere thanks to Drs. Enrique Saldaña, George Spirou, Donald Godfrey and Aric Agmon for their valuable pre-submission critiques of the manuscript.

This work was supported by research grant DC 02266 to ASB from the National Institute on Deafness and Other Communication Disorders, National Institutes of Health. RJK was supported by a graduate teaching assistantship from the West Virginia University Department of Anatomy. 


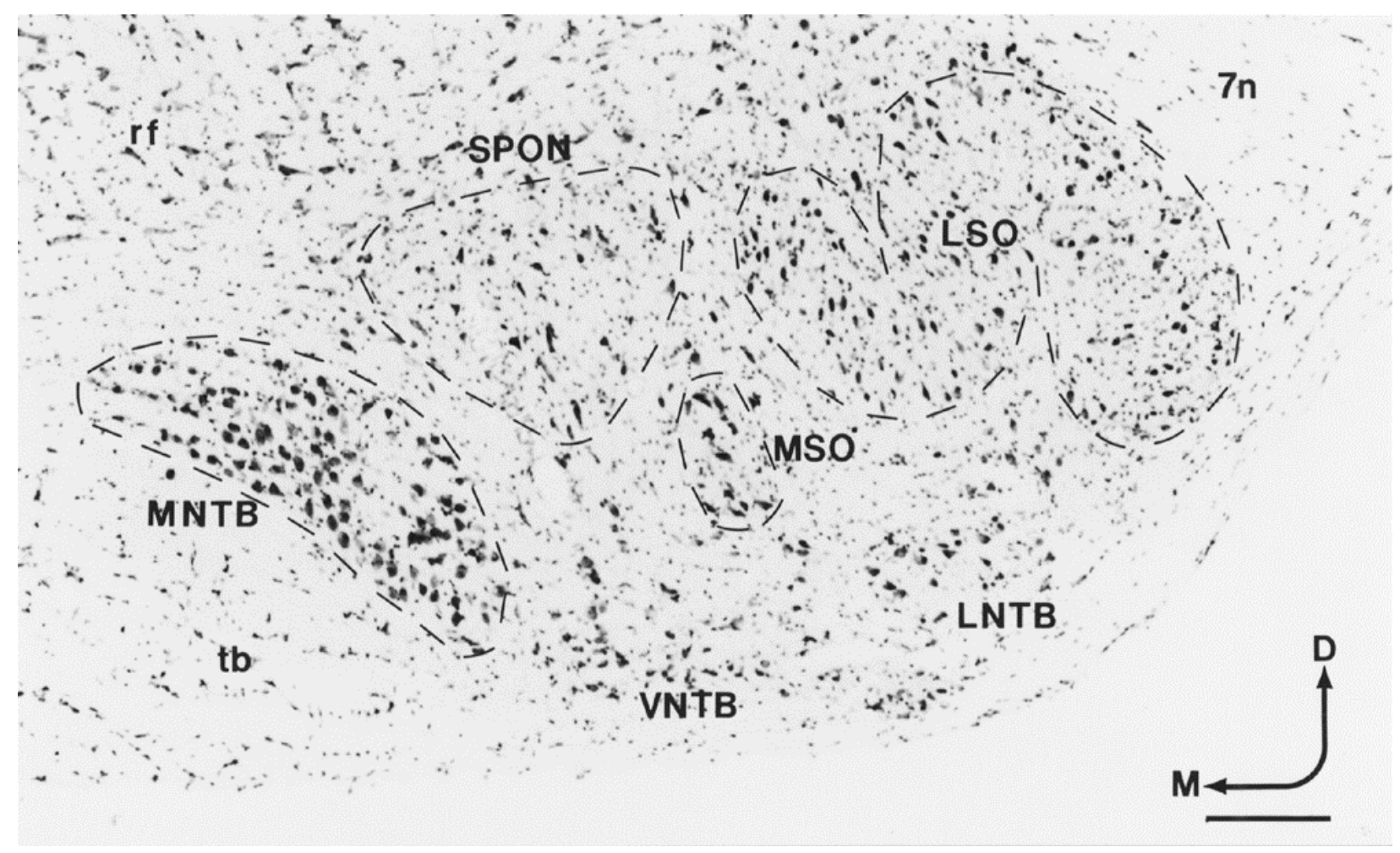

Figure 2.1. Organization of the rat superior olivary complex. Cresyl violet stained coronal section through the SOC at the level of the seventh cranial nerve (7n) root. The three principal nuclei of the SOC, the lateral superior olive (LSO), medial superior olive (MSO) and the medial nucleus of the trapezoid body (MNTB), can all be seen in this section, along with several periolivary nuclei. It is evident that the superior paraolivary nucleus (SPON) has a relatively low density of neurons. D, dorsal; LNTB, lateral nucleus of the trapezoid body; M, medial; rf, pontine reticular formation; tb, trapezoid body; VNTB, ventral nucleus of the trapezoid body. Scale bar $=200 \mu \mathrm{m}$ 


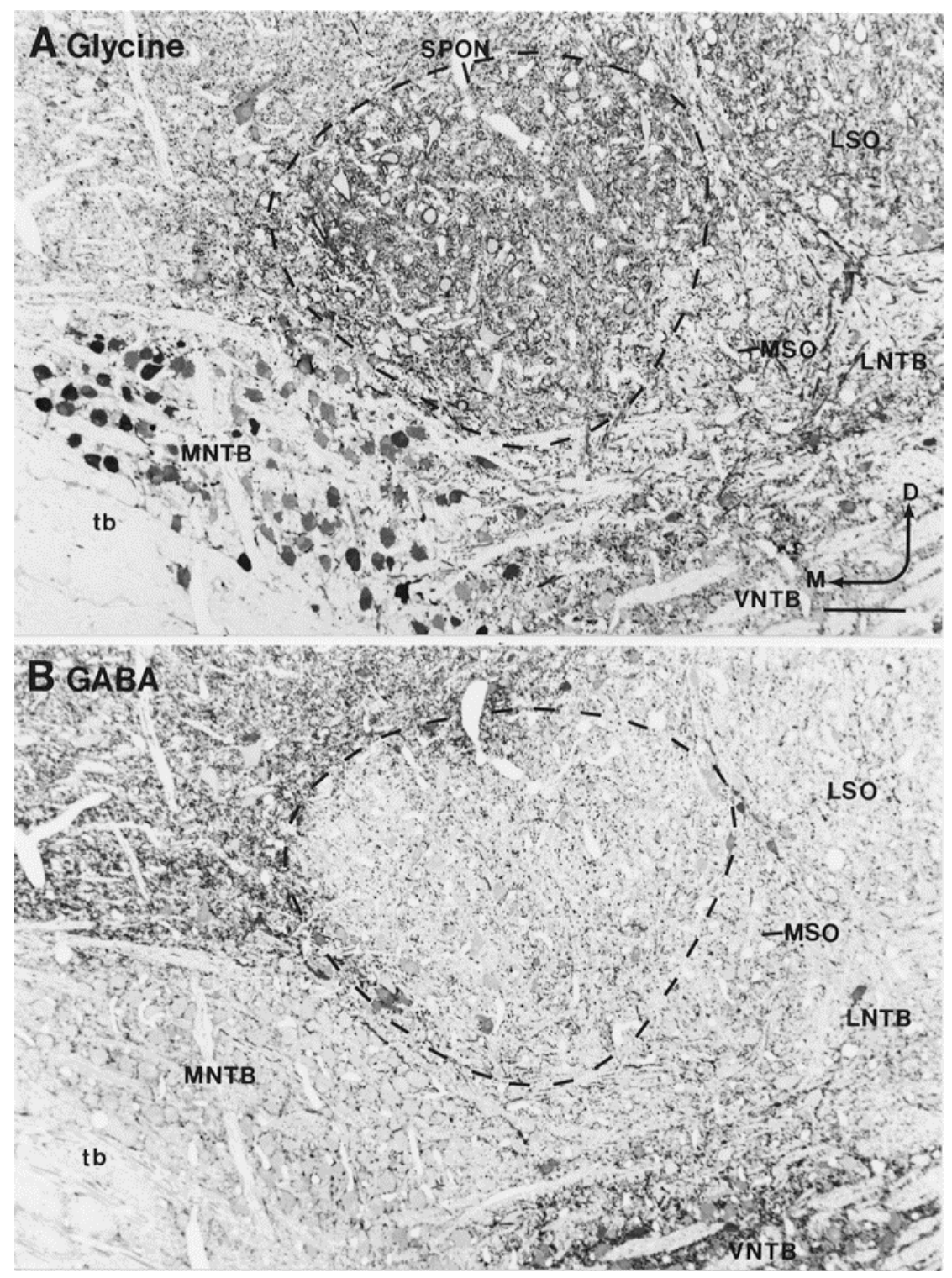

Figure 2.2. Overview of glycine-IR and GABA-IR in the rat SOC. Postembedding immunocytochemistry performed on plastic embedded, semi-thin sections demonstrate glycine-IR and GABA-IR within SOC nuclei. (A) In glycine material, MNTB neurons are easily recognized by their darkly immunostained somata. The SPON contains only rare glycine-IR cell bodies but the neuropil reveals a high density of glycine-IR fibers and punctate profiles. (B) In sections processed to reveal GABA, cell bodies and neuropil in VNTB are the most prominently immunolabeled. SPON contains numerous GABAergic cell bodies, most of which are lightly to moderately immunolabeled. GABA-IR punctate profiles are distributed throughout the neuropil of SPON. Abbreviations as in Figure 1. Scale bar $=100 \mu \mathrm{m}$ 


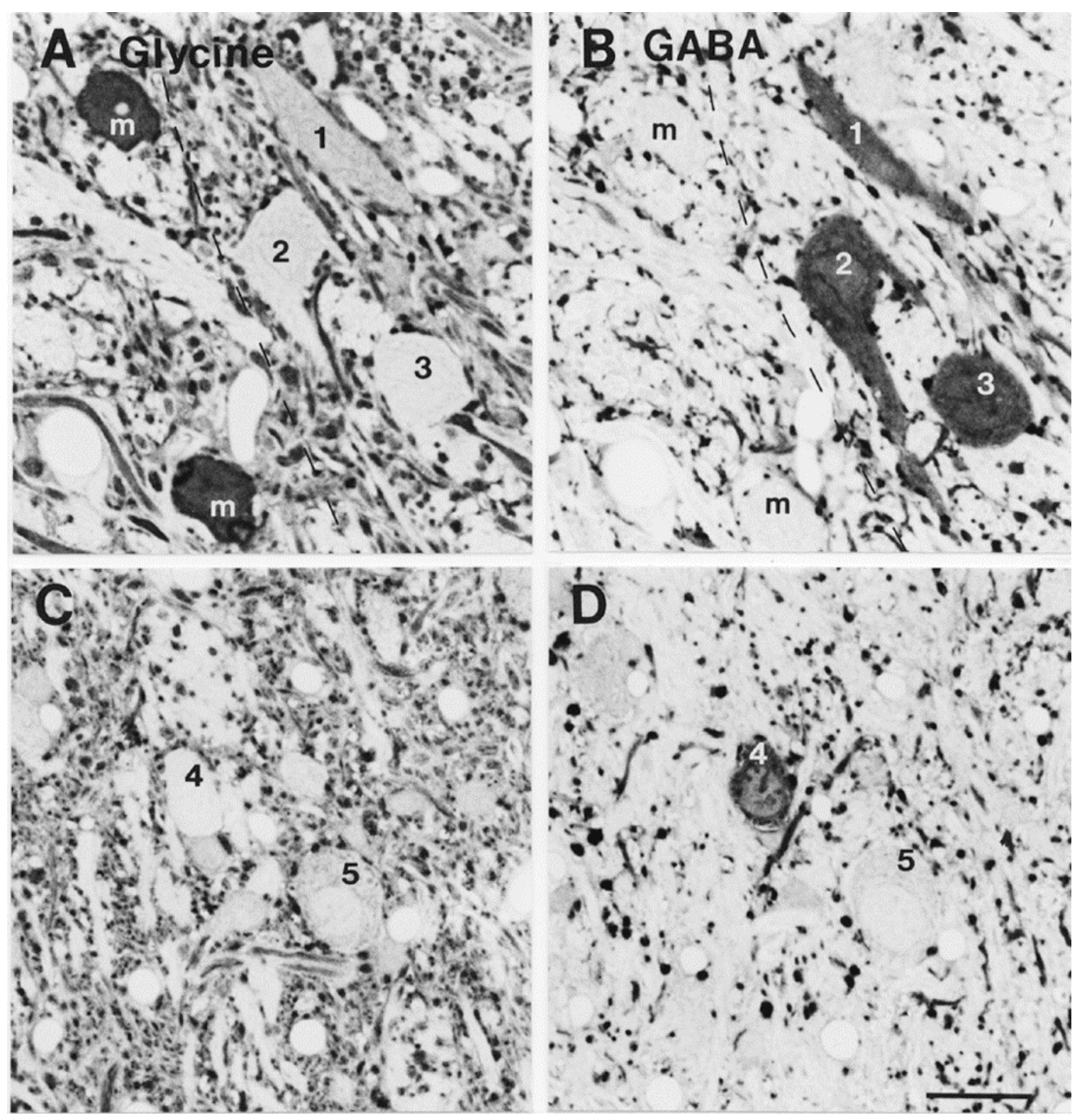

Figure 2.3. Glycine-IR and GABA-IR of the SPON neurons. High magnification photomicrographs of coronal semithin sections show the range of immunolabeling intensities observed in SPON and MNTB. (A-B) Five neurons are shown, and the dashed line denotes the boundary between MNTB and SPON. The two MNTB neurons (m) are distinctly glycine-IR and GABA-immunonegative. One of the SPON cells (1) displays light glycine-IR while all three are intensely GABA-IR. (C-D) Two additional SPON cells are illustrated. Cell 4 is glycineimmunonegative and GABA-IR. Cell 5 is lightly glycine-IR and GABA-immunonegative. Scale bar $=20 \mu \mathrm{m}$. 

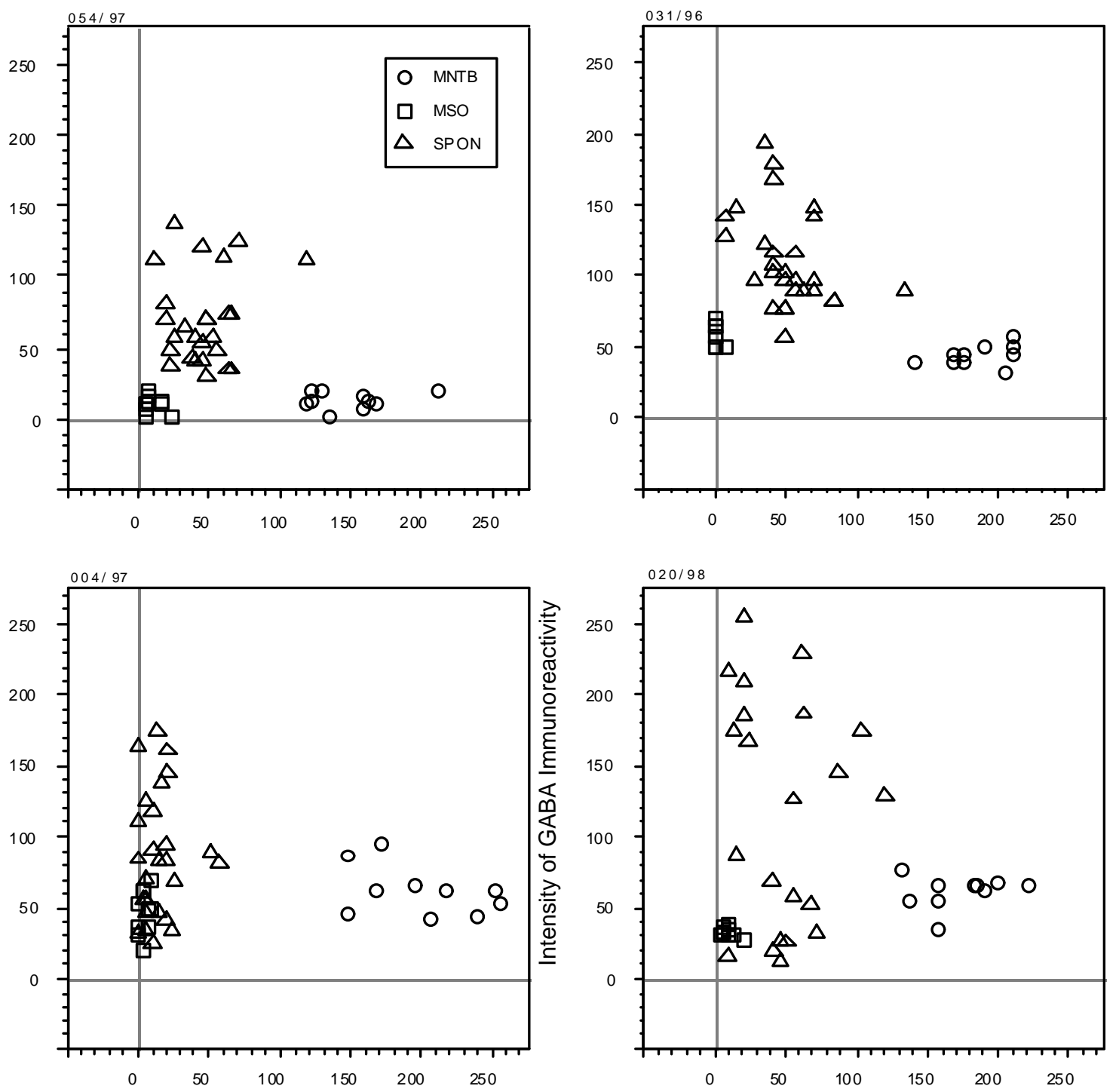

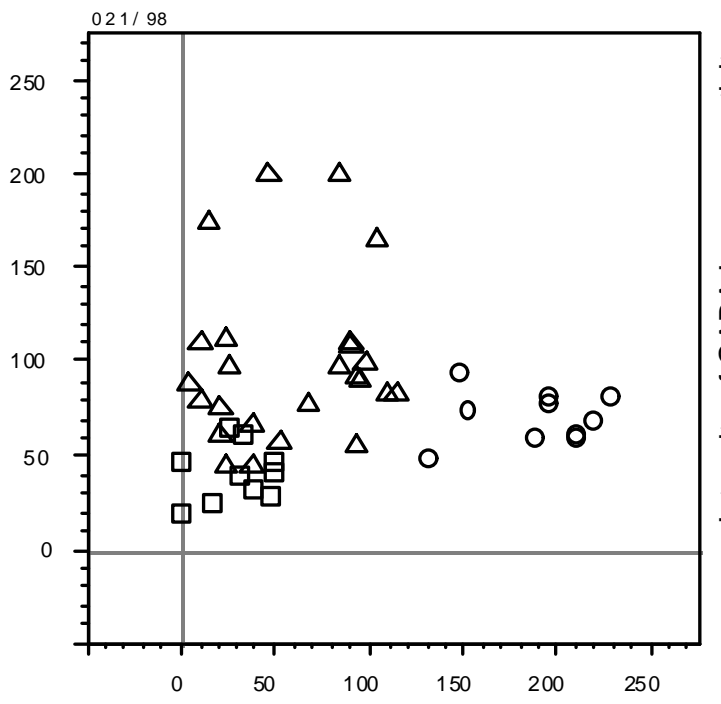

Intensity of Glycine Immunoreactivity

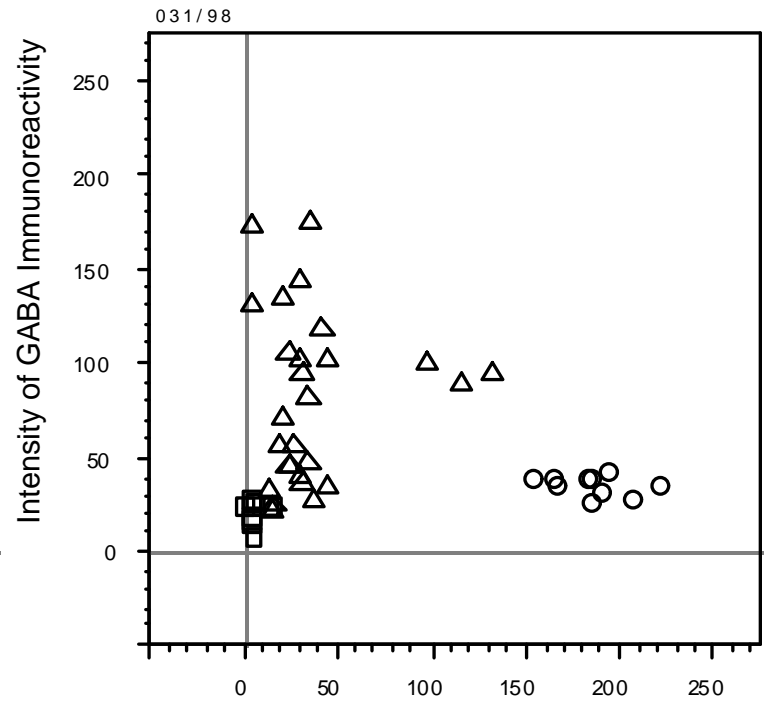

Intensity of Glycine Immunoreactivity 
Figure 2.4. Assessment of glycine-IR and GABA-IR intensities in somata of the SOC. The same neurons were immunolabeled with antiserum directed against glycine or GABA in adjacent semithin sections from six animals. IR intensity values for MSO cells (squares) cluster at the lower end of both intensity scales. In contrast, the IR intensity values representing MNTB neurons (circles) cluster at the high end of the glycine-IR and the low end of the GABA-IR scales. SPON neurons (triangles) show a wide range of GABA-IR intensities, and are the most intensely immunoreactive for GABA among the three nuclei. Glycine-IR values of SPON neurons are mostly distributed within the lower third of the glycine-IR intensity scale. The number at the top left of each panel indicates the experimental animal identification number. The sections from all animals were incubated with the commercial glycine antiserum (Chemicon) and the GABA antiserum provided by Dr. David Pow, except those from animal 054/97 (top left panel) which was incubated with the commercial GABA antiserum (HTI Bioproducts). In animals 054/97 and 031/96, but not the remaining cases, the antisera were pre-absorbed as described in Methods. 


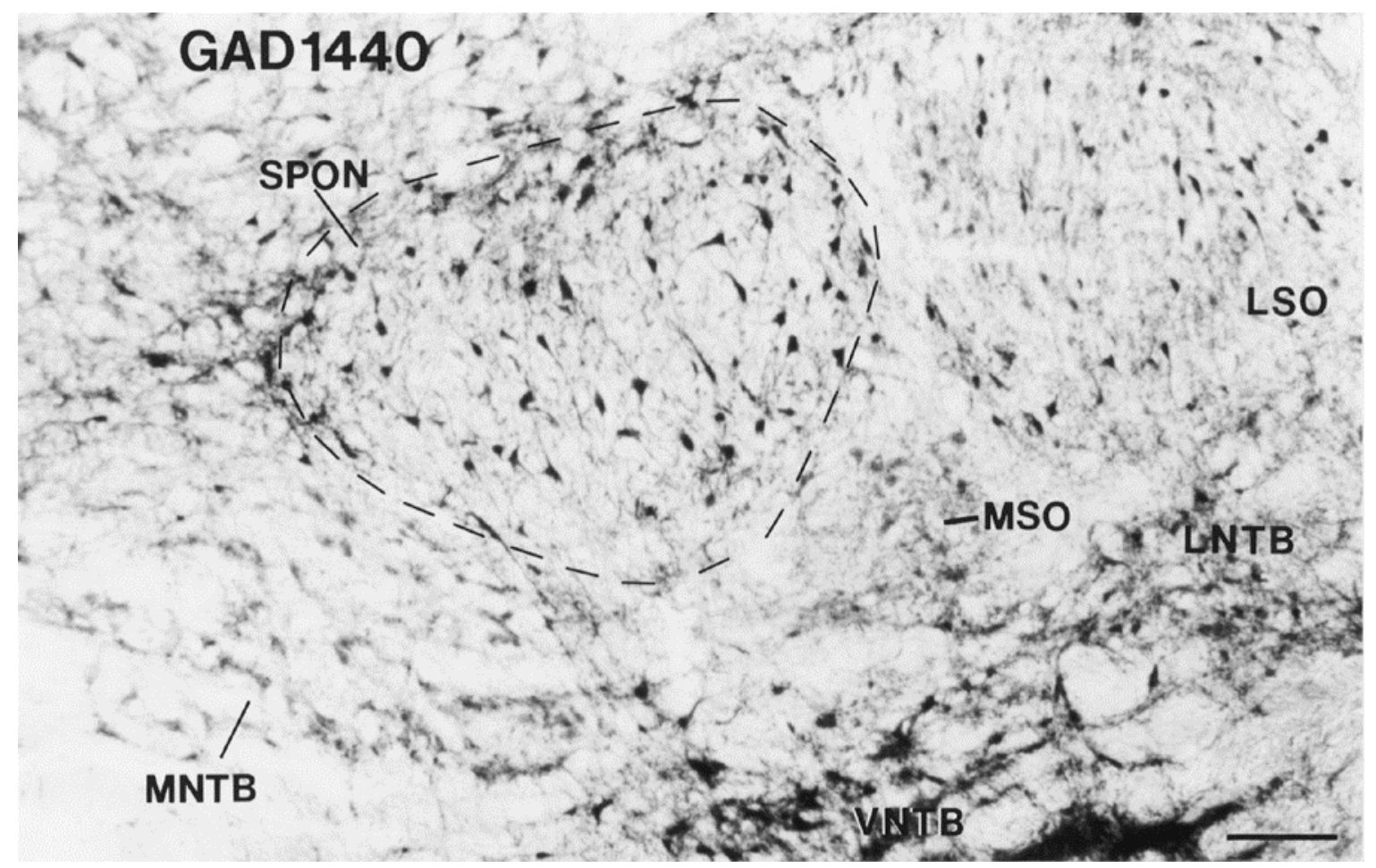

Figure 2.5. Immunoreactivity for glutamic acid decarboxylase (GAD) in the SOC. Frozen coronal tissue section processed using the GAD-1440 antiserum, which cross-reacts with both isoforms of GAD. The LSO, SPON, VNTB and LNTB contain GAD-IR neurons. MNTB and MSO neurons are GAD-immunonegative, but receive GAD-IR innervation which outlines their cell bodies and proximal dendrites. In the coronal plane, most SPON neurons appear bipolar, although round or multipolar forms are also seen occasionally. Scale bar $=100 \mu \mathrm{m}$ 


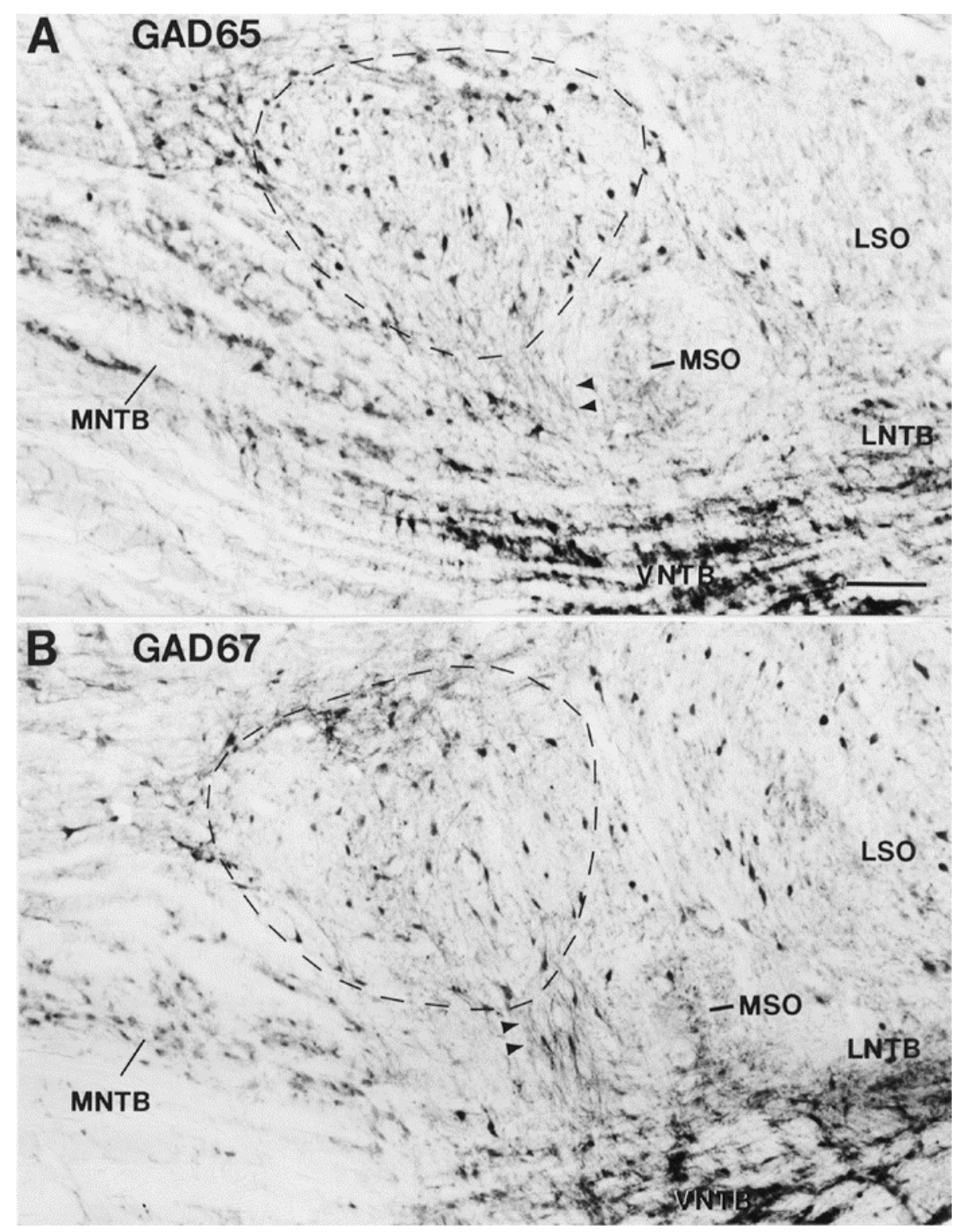

Figure 2.6. GAD 65-IR and GAD 67-IR in the SOC. Coronal sections illustrate the distribution of GAD isoforms in the SOC as revealed by antisera specifically directed against either GAD 65 (A) or GAD 67 (B). Immunoreactive neurons are present in SPON, VNTB and LNTB. Fewer LSO neurons were immunolabeled for GAD 65 than for GAD 67. GAD-IR dendrites (arrowheads) appear to extend between the ventral SPON and the VNTB. Scale bar $=100 \mu \mathrm{m}$ 


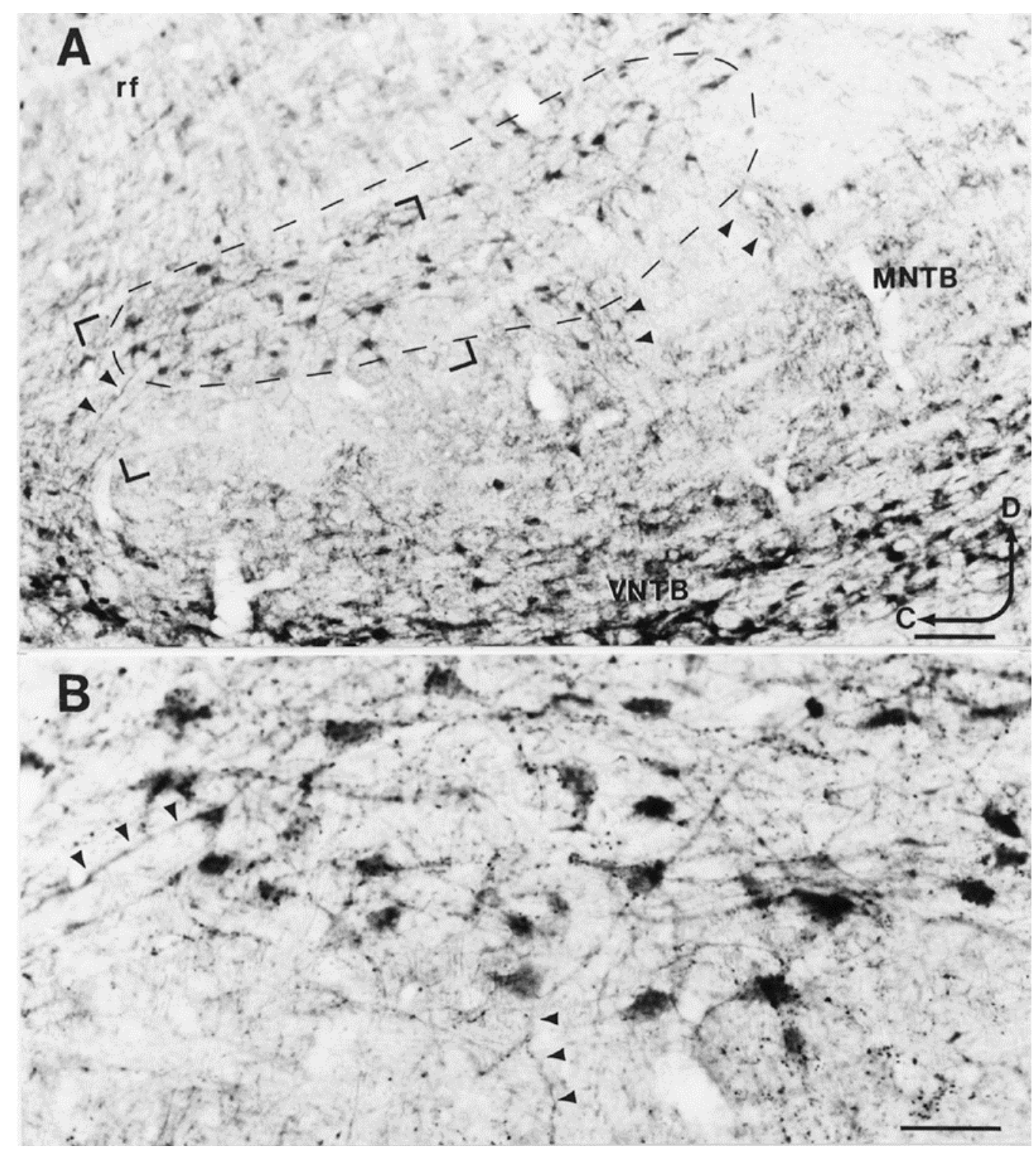

Figure 2.7. GAD-IR in a parasagittal section through the SOC. Frozen parasagittal tissue section processed according to the PAP method using GAD 65 antiserum. At this level through the SOC, portions of the VNTB, MNTB and SPON (outlined by dashed border) are visible. Bundles of GAD-IR dendrites (arrowheads) are separated by immunonegative fascicles of trapezoid body fibers. (B) Higher magnification photomicrograph of the bracketed region in (A). SPON neurons appear distinctly multipolar when sectioned parasagittally. Note also the dense axosomatic and axo-dendritic GAD-IR innervation of these neurons. In some cases, the ventrally directed GAD-IR dendrites (arrowheads) could be traced to their parent cell bodies in the SPON. C, caudal; D, dorsal; rf, pontine reticular formation. Scale bar $=100 \mu \mathrm{m}$ in A, $50 \mu \mathrm{m}$ in B. 


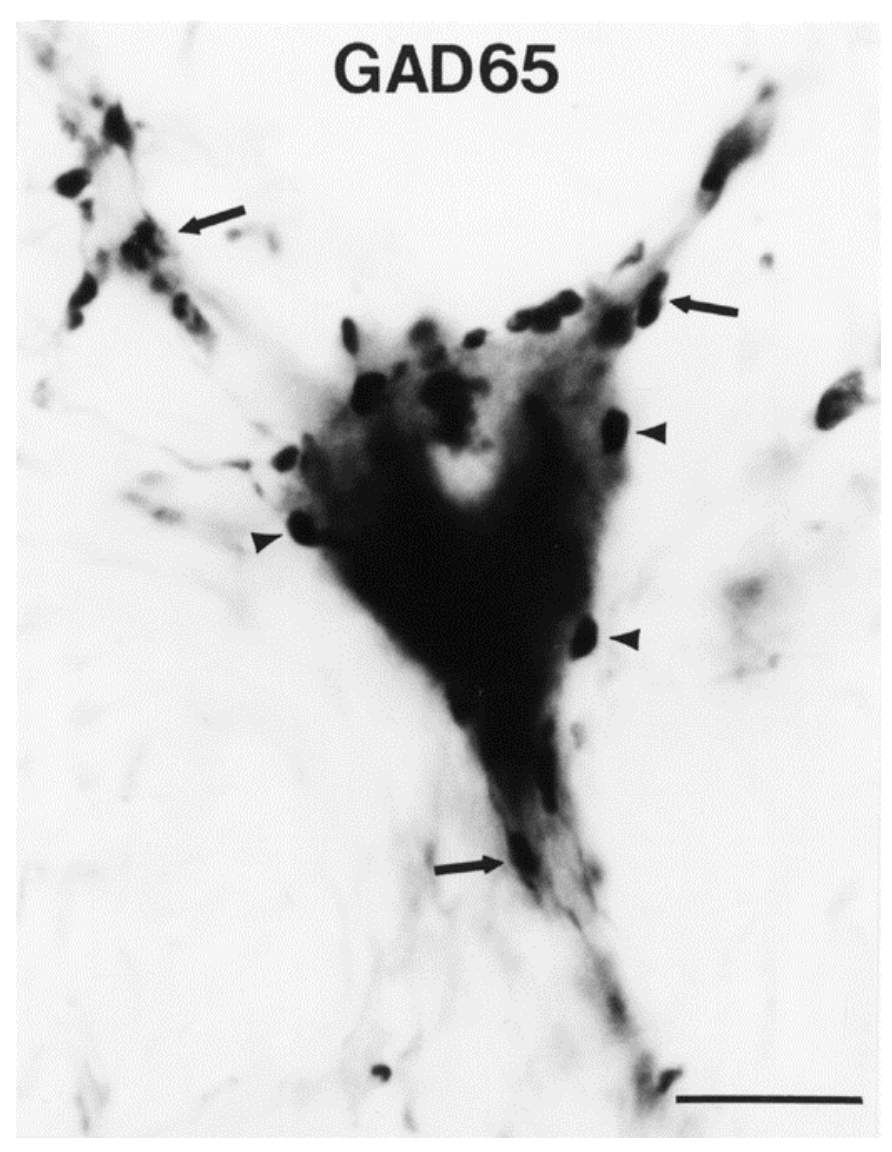

Figure 2.8. GAD-IR puncta apposed to a GAD-IR neuron in the SPON. SPON neuron displaying a distinct multipolar morphology and strongly expressing the $65 \mathrm{kD}$ isoform of GAD. Numerous GAD 65-IR punctate profiles can be seen apposed to the soma (arrowheads) and dendrites (arrows) of this cell. Scale bar $=10 \mu \mathrm{m}$. 
Table 2.1. Classification of Glycine and GABA immunoreactivities in SOC neurons.

\begin{tabular}{|c|c|c|c|c|c|c|}
\hline \multirow[t]{2}{*}{ Nucleus } & \multicolumn{3}{|c|}{$\begin{array}{l}\text { Glycine-IR intensity } \\
\text { Proportion of cells }\end{array}$} & \multicolumn{3}{|c|}{$\begin{array}{l}\text { GABA-IR intensity } \\
\text { Proportion of cells }\end{array}$} \\
\hline & $\begin{array}{l}\text { Neg. - Light } \\
(\mathrm{OD}=0-85)\end{array}$ & $\begin{array}{c}\text { Moderate } \\
(\mathrm{OD}=85-170)\end{array}$ & $\begin{array}{c}\text { Dark } \\
(\mathrm{OD}=170-255)\end{array}$ & $\begin{array}{l}\text { Neg. - Light } \\
(\mathrm{OD}=0-85)\end{array}$ & $\begin{array}{c}\text { Moderate } \\
(\mathrm{OD}=85-170)\end{array}$ & $\begin{array}{c}\text { Dark } \\
(\mathrm{OD}=170-255)\end{array}$ \\
\hline $\begin{array}{l}\text { MNTB } \\
(n=60)\end{array}$ & $0 \%$ & $43 \%$ & $57 \%$ & $95 \%$ & $5 \%$ & $0 \%$ \\
\hline $\begin{array}{l}\text { MSO } \\
(n=60)\end{array}$ & $100 \%$ & $0 \%$ & $0 \%$ & $100 \%$ & $0 \%$ & $0 \%$ \\
\hline $\begin{array}{l}\text { SPON } \\
(n=154)\end{array}$ & $89 \%$ & $11 \%$ & $0 \%$ & $49 \%$ & $41 \%$ & $10 \%$ \\
\hline
\end{tabular}


Table 2.2. Unbiased estimates of Nissl stained and GAD-IR neurons in the rat SPON

\section{Estimated number of neurons}

\begin{tabular}{clll} 
Animal \# & Isoform & GAD & Nissl \\
\hline $002 / 97 \mathrm{a}$ & & 2,398 & \\
$002 / 97 \mathrm{~b}$ & GAD65\&67 & 2,158 & 2,278 \\
\hline $037 / 98$ & GAD65 & 2,314 & \\
\hline $024 / 98$ & GAD67 & 2,346 & \\
\hline $005 / 94$ & - & & 2,499 \\
$035 / 94$ & - & & 2,611 \\
\hline Overall estimates & 2,313 & 2,555
\end{tabular}

To verify the reliability of the unbiased stereology method in our material, two separate estimates of GAD-IR neurons were obtained from animal 002/97 using the GAD-1440 antiserum. The number of GAD-IR neurons were then estimated from two other animals using either the GAD-6 or the K2 antiserum. We also estimated the number of Nissl stained cells in the SPON of two additional animals. Approximately $90 \%$ of SPON neurons are GAD-IR, and each antiserum reveals essentially the same number of cells. 


\section{Table 2.3. Morphometric analysis of GAD labeled SPON neurons}

\begin{tabular}{|c|c|c|c|c|c|}
\hline & & & Mean & Mean & \\
\hline $\begin{array}{l}\text { Plane of } \\
\text { section }\end{array}$ & $\begin{array}{l}\text { Mean Area } \\
\left(\mu \mathrm{m}^{2} \pm \text { S.E. }\right)\end{array}$ & $\begin{array}{c}\text { Mean } \\
\text { Perimeter } \\
(\mu \mathrm{m} \pm \text { S.E. })\end{array}$ & $\begin{array}{l}\text { Maximum } \\
\text { Diameter } \\
(\mu \mathrm{m} \pm \text { S.E. })\end{array}$ & $\begin{array}{l}\text { Minimum } \\
\text { Diameter } \\
(\mu \mathrm{m} \pm \text { S.E. })\end{array}$ & $\begin{array}{c}\text { Mean } \\
\text { Circularity } \\
\text { (average } \pm \\
\text { S.E.) }\end{array}$ \\
\hline $\begin{array}{l}\text { Coronal } \\
(\mathrm{n}=138)\end{array}$ & $184.89 \pm 5.74$ & $63.69 \pm 1.22$ & $21.51 \pm 0.43$ & $10.90 \pm 0.24$ & $0.578 \pm 0.01 *$ \\
\hline $\begin{array}{l}\text { Sagittal } \\
(\mathrm{n}=108)\end{array}$ & $335.90 \pm 10.64 *$ & $113.81 \pm 2.42 *$ & $29.64 \pm 0.71^{*}$ & $14.61 \pm 0.39 *$ & $0.336 \pm 0.01$ \\
\hline
\end{tabular}

GAD-immunoreactive cell bodies were larger and less circular when sectioned parasagitally than when sectioned coronally, supporting the conclusion that SPON cell bodies are multipolar, elongated rostrocaudally and flattened parasagittally. 


\section{Chapter Three}

\section{Unbiased stereological estimates of neuron number in subcortical auditory nuclei of the rat}

This work is published: Hearing Research 3903 (2002) 1-12 


\begin{abstract}
The mammalian auditory system consists of a large number of cell groups, each containing its own complement of neuronal cell types. In recent years, much effort has been devoted to the quantitation of auditory neurons with common morphological, connectional, pharmacological or functional features. However, it is difficult to place these data into the proper quantitative perspective due to our lack of knowledge of the number of neurons contained within each auditory nucleus. To this end, we have employed unbiased stereological methods to estimate neuron number in the cochlear nuclei, superior olivary complex, lateral lemniscus, inferior colliculus and medial geniculate body. Additionally, we generated a three-dimensional model of the superior olivary complex. The utility of unbiased stereological estimates of auditory nuclei is discussed in the context of various experimental paradigms.
\end{abstract}




\section{INTRODUCTION}

The structural complexity of the mammalian auditory system has been appreciated since the early anatomists described the numerous nuclei that comprise the ascending pathways, and also the great diversity of their constituent neurons' morphologies. Modern auditory neurobiology relies heavily on the quantitative analyses of structure and function. However, limited knowledge of the structural composition of auditory nuclei renders the data produced in many studies difficult to interpret. To date, only a few investigations have attempted to determine the number of neurons in nuclei of the central auditory system (see discussion), and most of these have relied on biased and therefore potentially unreliable counting methods. Therefore, a systematic investigation of neuron numbers in the central auditory system of a mammalian species is warranted.

The goal of this study was to provide unbiased, accurate estimates of the total number of neurons in subcortical auditory nuclei of the rat. This species is commonly used for physiological and anatomical investigations, and substantial background information pertaining to its central auditory system is available (reviewed in Webster, 1995). Knowledge of the number of neurons located in auditory nuclei, coupled with an understanding of their physiological response properties, connectivities and neurochemical phenotypes will enable the formation of accurate quantitative models of auditory circuits. This information may also prove useful in a number of other ways. Knowing the number of cells located in an auditory nucleus enables an assessment of the percentage of cells that are visualized by selective labeling methods such as immunocytochemistry, in situ hybridization, tract-tracing or experimental degeneration approaches. For instance, immunocytochemical probes commonly reveal a single neuron type in a particular brain region, and occasionally even a sub-population of a cell type that expresses the marker under investigation. Well documented examples include the cartwheel cells of the dorsal cochlear nucleus (DCN), which represent the only neurons in the guinea pig cochlear nuclear complex that are immunoreactive for PEP-19 (Berrebi and Mugnaini, 1991), or the cartwheel cells of the rat DCN, only a percentage of which are glycinergic (Gates et al., 1996). Similarly, in tract-tracing experiments the percentage of retrogradely labeled cells in a given nucleus is influenced by the number of its neurons that actually participate in the pathway under study, and 
by various technical artifacts resulting from the effective size of the injection site and the efficiency of the retrograde transport of the chosen tracer. Thus, only with knowledge of the entire population of cells contained within the structure under study can one make quantitative assessments of cells expressing a particular marker or projecting to a particular brain region. For example, by combining immunohistochemical and retrograde tracing data with unbiased stereological estimates of neuron number, it has recently been demonstrated that greater than $90 \%$ of neurons in the superior paraolivary nucleus (SPON) express glutamic acid decarboxylase and project to the ipsilateral inferior colliculus (Saldaña and Berrebi, 2000; Kulesza and Berrebi, 2001).

Design-based stereology is a technique that provides reliable estimates of the number and properties of neurons (Howard and Reed, 1998). The method has a rigorous mathematical foundation and provides an indication of the precision of the estimates obtained. Stereologicallybased estimates are considered 'unbiased' if the chosen test probe is employed using appropriate rules for systematic random sampling of the structure of interest. Once the so-called sampling and systematic sources of bias are eliminated, no assumptions need to be made about the size, shape or distribution of the elements under study. Implementing unbiased stereology is largely automated, thus maximizing efficiency and eliminating human errors. Therefore, we have applied design-based stereology to determine the number of neurons present in the cochlear nuclei, superior olivary complex, lateral lemniscal nuclei, inferior colliculus and medial geniculate body of the rat. We also produced a three-dimensional structural model of the superior olivary complex in this species. 


\section{MATERIALS AND METHODS}

\section{Animals and Tissue Processing}

Adult female Wistar rats (190-210 g body weight) were anesthetized with an intraperitoneal overdose of sodium pentobarbital $(120 \mathrm{mg} / \mathrm{kg}$ body weight $)$ and perfused through the ascending aorta with a vascular rinse composed of calcium-free Ringer's solution ( $\mathrm{pH} \mathrm{6.9).}$ Fixation was accomplished first with $4 \%$ freshly depolymerized paraformaldehyde and $0.1 \%$ glutaraldehyde in $0.1 \mathrm{M}$ phosphate buffer, followed by $4 \%$ freshly depolymerized paraformaldehyde in the same buffer. Brains were then dissected and cryoprotected in $30 \%$ sucrose in saline. Sectioning was performed on a freezing microtome at a thickness of $40 \mu \mathrm{m}$. Sections were mounted in serial order onto glass slides, air-dried, stained for Nissl substance using $1 \%$ cresyl violet and coverslipped with Entellan (Merck).

\section{Unbiased Stereology}

We selected for study three cases from our collection of cresyl violet-stained series of rat brain sections. This material was chosen because cresyl violet staining reveals all cells and provides good resolution of the cytological features of neurons and glial cells.

Using two coronal series of sections, we estimated the number of neurons contained in nineteen auditory structures (nuclei or subdivisions) bilaterally. Because the precise rostral and caudal borders of certain superior olivary complex (SOC) nuclei were sometimes difficult to discern in coronal sections, we also counted neurons in SOC nuclei from one parasagittal series of sections. Stereo Investigator software (MicroBrightfield, Inc., Colchester, VT) was used to implement the optical fractionator protocol, which encompasses the optical disector and fractionator tools (Gundersen, 1988, Gundersen et al., 1988). In order to minimize the coefficient of error, we counted at least 100 neurons and sampled from ten or more sections in each auditory structure. To begin, we randomly selected a section containing the cell group of interest and then sampled uniformly spaced sections, the frequency of which depended on the size of the structure (section fraction). Images of each selected section were captured by video 
camera and displayed on a computer monitor. For every chosen section, a contour enclosing each structure was traced on the monitor using a $4 \mathrm{X}$ or $10 \mathrm{X}$ objective lens. To select the sample from each contour drawn, the software created a two-dimensional counting frame (measuring $4,900 \mu \mathrm{m}^{2}$ ) formed by two inclusion lines and two exclusion lines, and this frame was used to sample from approximately twenty sites within each contour (area fraction). The thickness of each tissue section was determined to be between 20 and $23 \mu \mathrm{m}$ by averaging the thickness measured from several locations using a $100 \mathrm{X}$ oil immersion objective lens (NA 1.35; final magnification on the screen was 2,680X). For stereological counts, the top $5 \mu \mathrm{m}$ and the bottom $5-8 \mu \mathrm{m}$ (depending on the actual thickness) of the tissue sections were reserved as guard zones (disector fraction) and only neurons that 1) first came into focus between the guard zones and 2) did not intersect the forbidden boundary lines of the counting frame were included in the counts (number of counted cells). Cellular profiles displaying cytoplasmic staining of Nissl granules surrounding a pale nucleus were classified as neurons and were included in our counts. Cells were identified as glia if they displayed limited cytoplasm and darkly-stained nuclei and were excluded from our counts. The very numerous granule cells of the cochlear nuclei were also excluded from our counts because they were often difficult to distinguish from small glial cells. In one cursory examination, we estimated that more than 50,000 granule cells reside within the dorsal cochlear nucleus alone (DCN), which roughly coincides with a recent study of the mouse DCN using the optical fractionator (Idrizbegovic et al., 2001). Therefore, it must be noted that the number of neurons involved in auditory processing in the cochlear nuclei is much higher than the numbers presented here.

Our final neuronal estimates $\left(\boldsymbol{N}_{\text {est }}\right)$ were calculated using the equation:

$$
\boldsymbol{N}_{\text {est }}=\text { number of counted cells } \mathrm{X} \text { area fraction } \mathrm{X} \text { section fraction } \mathrm{X} \text { disector fraction }
$$

The three-dimensional reconstruction of the SOC was created with NeuroLucida and NeuroExplorer software (MicroBrightField, Inc., Colchester, VT) by stacking the contours for each nucleus and factoring in the appropriate spacing between sections before shrinkage. 
All animal procedures were approved by the Institutional Animal Care and Use Committee of West Virginia University, in accordance with existing regulations of the National Institutes of Health. 


\title{
RESULTS
}

\section{Stereological Estimates of Neuron Number}

\author{
Cochlear Nuclei
}

The cochlear nuclei $(\mathrm{CN})$ were subdivided into the anteroventral cochlear nucleus (AVCN), the posteroventral cochlear nucleus (PVCN) and the dorsal cochlear nucleus (DCN) as depicted in Figure 3.1. In the case of this large complex, delineating the boundaries of the three nuclei was easily accomplished by using the entry of the eighth nerve root, the taenia choroidea and the granule cell lamina as guides (Osen, 1969, 1988; Brawer et al., 1974; Mugnaini et al., 1980; Lorente de Nó, 1981). The counts from each subdivision are provided in Table 3.1. We estimate that the rat cochlear nucleus contains a total of 30,300 neurons (not including granule cells), with $42 \%$ located in the AVCN with the remaining neurons roughly equally distributed between the PVCN and DCN.

\section{Superior Olivary Complex}

Six structures within the superior olivary complex (SOC) were examined. Our determination of the borders of these nuclei is shown in a series of coronal sections in Figure 3.1 and in parasagittal sections in Figure 3.2. In most sections, the borders of the medial nucleus of the trapezoid body (MNTB), superior paraolivary nucleus (SPON) and medial superior olive (MSO) were relatively easily discerned by the distinct morphologies, arrangement and/or orientation of neurons contained in each nucleus. The small MSO is identified as a narrow vertical column of horizontally-oriented bipolar neurons. We noted that in the rostral-most SOC, the MSO expands dorso-ventrally and its cross-sectional area nearly triples in size. In coronal sections through the middle of the SOC (Fig. 3.1D), the lateral superior olive (LSO) is readily recognized by its S-shape and its tightly packed bipolar neurons. However, the LSO is considerably shorter in the rostro-caudal dimension than the other SOC nuclei (Fig. 3.2) and is not present in rostral-most (Fig. 3.1C) or caudal-most sections (Fig. 3.1E).

The borders of the other two structures examined (the ventral and lateral nuclei of the 
trapezoid body [VNTB and LNTB, respectively]), were difficult to define in some coronal sections, particularly at their rostral and caudal ends. The LNTB contains neurons with relatively large somata and darkly stained Nissl substance as compared to the smaller, paler staining neurons that comprise the LSO. This feature was useful in distinguishing these two structures, especially in rostral sections where the LNTB extends dorsally and occupies a position similar to that of the more caudal LSO. The VNTB is located ventral to the MSO and SPON and is composed of small, sparsely packed neurons situated among trapezoid body fibers. This nucleus extends quite far rostrally into the midbrain, where it occupies a position very close to the caudal tip of the VNLL (Fig. 3.2A).

According to our estimates derived from coronal sections, approximately 18,600 neurons are located in the six SOC nuclei combined (Table 3.1). The MNTB and VNTB, with 6,000 neurons and 4,500 neurons respectively, account for more than half of all SOC neurons. The SPON, LSO and LNTB each contain between 2,100 and 2,500 neurons, and 1,100 neurons are found in the MSO.

The neuronal estimates for the MNTB, LSO and SPON obtained from the single parasagittal series of sections were within $10 \%$ of the average estimate derived from the two coronal series. However, greater discrepancies were observed for those structures whose borders were the most difficult to identify in rostral coronal sections, namely the LNTB, MSO and VNTB (18\%, 19\% and 28\% higher estimates derived from parasagittal than from coronal sections, respectively).

\section{Lateral Lemniscus}

We estimated the number of neurons in the ventral and dorsal nuclei of the lateral lemniscus (VNLL and DNLL), and in two adjacent structures: the nucleus sagulum and the horizontal cell group (HCG) (Fig. 3.3). The HCG is a group of small horizontally oriented and loosely packed neurons situated between the DNLL and the VNLL (Ruiz-Gómez, 1988; Bajo et al., 1993; Caicedo and Herbert, 1993; Merchán et al., 1994). Altogether, these four structures contain approximately 17,500 neurons, and by our estimate nearly $80 \%$ are situated in the 
VNLL.

Inferior Colliculus

We have adopted the parcellation of the inferior colliculus (IC) proposed by Saldaña et al. (1996). According to these authors, the IC consists of the medial IC (IC-m), which includes the more commonly named dorsal cortex and central nucleus, and the lateral IC (IC-1), similar to the external cortex of earlier subdivision schemes. The divisions of the IC are shown in Figure 4 , and the estimates of cell number in these subdivisions are given in Table 3.1. We have also estimated the number of neurons interspersed among the fibers of the brachium of the IC (bic). Taken together, these auditory mesencephalic structures contain some 373,600 neurons, with approximately $60 \%$ located in the IC-m, $35 \%$ found in the IC-1 and $5 \%$ in the bic.

\section{Medial Geniculate Body}

We parcelled the rat medial geniculate body (MGB) into three subdivisions: ventral (MGBv), dorsal (MGBd) and medial (MGBm) (LeDoux et al., 1987; Winer et al., 1999a, b). We chose to exclude the so-called suprageniculate nucleus (LeDoux et al., 1987) because its involvement in auditory circuits and processing has not been firmly demonstrated. Figure 3.5 shows the three subdivisions of the MGB at two different rostro-caudal levels. As a whole, the MGB contains approximately 72,300 neurons, 65\% of which are located in the MGBv (Table 3.1).

\section{Three-Dimensional Reconstructions}

Figure 3.6 depicts the three-dimensional renderings of the six SOC nuclei examined. The reconstructions convey the complex geometries of these tightly packed cell groups in a manner that is difficult to appreciate in two-dimensional views. In particular, individual coronal sections through rostral levels of the SOC can lead to the confusion of the LNTB with the LSO and the VNTB with the VNLL. The reconstructions also clearly demonstrate the dorsal expansion of the MSO at rostral levels. This model can be rotated to achieve any view, which clarifies the 
borders of these structures as seen in any of the cardinal planes of section, or for that matter any oblique plane. The interested reader can access these reconstructions on the internet by visiting the website of the Sensory Neuroscience Research Center of West Virginia University (http://www.hsc.wvu.edu/snrc/berrebi.html). 


\section{DISCUSSION}

\section{Choice of the optical fractionator tool}

The field of stereology encompasses more than a dozen so-called "tools" for the study of biological entities or other particulate structures. When properly applied, each of these tools renders quantitative estimates that are efficiently obtained, accurate, and unbiased (Howard and Reed, 1998). We selected the optical fractionator method in our study because this particular tool is relatively simple, is unaffected by tissue shrinkage and can be applied to tissues processed by most, if not all, conventional techniques. Moreover, to employ the optical fractionator requires knowledge of only the number of sections containing the area of interest and the actual tissue thickness, but not the structure volume. Perhaps most importantly, since all elements are equally likely to be sampled using this procedure, the optical fractionator is considered to be "assumption-free", rendering the size, shape, orientation and spatial distribution of the neurons irrelevant. This represents a notable feature of this methodology, given the myriad different cell types within the numerous nuclei of the central auditory system.

Unbiased neuronal estimates may prove crucial to the interpretation of studies of convergence and/or divergence of neural pathways, as these represent important factors in the formation of quantitative neural circuit models. For stereology to be used precisely in this context, however, requires knowledge of not only the number of each population or class of neuron in the nuclei being investigated, but also of their afferent inputs and/or efferent projections. Given that the rodent cochlear nuclei alone contain nearly a dozen identified cell types (reviewed by Cant, 1992) such an undertaking was well beyond the scope of this study. Nonetheless, the simple procedures utilized herein can be applied to this end in the future.

\section{Consideration of plane of section}

We compared data from coronal and parasagittal sections of the SOC as an intrinsic control. The decision to include a parasagittal series of SOC sections was made in part because our neuronal estimates from coronal SOC sections differed from previously published estimates 
(see below), and in part because of the difficulty in discerning the rostral and caudal boundaries of certain SOC nuclei. This difficulty is demonstrated by the fact that the standard errors of our estimates were proportionally higher, on average, for SOC nuclei than for the other structures included in this study. For the MNTB, SPON and LSO, three nuclei that can be readily recognized by their shape, size and position and by the morphology, density and/or special arrangement of their neurons, the estimates obtained from coronal sections were very similar to those derived from parasagittal sections. However, the MSO, VNTB and LNTB, which display striking shifts and/or expansions in the rostral-most aspect of the SOC, appeared to contain 'more' neurons when sectioned parasagittally. As already mentioned, the estimates yielded by the optical fractionator should be unaffected by the orientation of the neurons and therefore should be similar in any plane of section. Consequently, we presume that the observed differences were due to the borders of these nuclei being delineated differently in the two planes of section. Since most nuclei of the SOC are elongated rostrocaudally, the topographical relationships between cell groups are better appreciated in the parasagittal or in the horizontal perspective. In fact, we analyzed in detail several series of horizontal sections through the SOC not used for the stereological counts, and these observations confirmed our interpretation of the boundaries of SOC nuclei derived from parasagittal sections.

\section{Comparison with previous estimates of neuron number in auditory nuclei of the rat}

Only a few previously published papers provide neuronal estimates for auditory nuclei in rats (Table 2). Agar and colleagues (1999) employed a stereological tool called the fractionator (not to be confused with the optical fractionator) to estimate neuronal number in the $\mathrm{CN}$ of Wistar rats. Our estimate of $\sim 9,000$ cells in DCN is reasonably close to their report of 10,560 cells (both counts are exclusive of granule cells). However, we report that the PVCN and AVCN contain a combined 21,300 neurons compared to their estimate of 15,600 neurons. We cannot readily account for the discrepancy, and it is not clear whether the primary difference lies in the estimates obtained from the AVCN or PVCN, as these authors did not separate the two nuclei.

Several groups have counted neurons in the rat SOC, but did not use stereological methods. To study the effects of aging on neuron numbers, Casey (1990) simply counted cell 
nuclei in rats between the ages of 3 and 30 months, using Abercrombie's (1946) correction factor to account for over-estimation due to split nuclei errors. As shown in Table 2, their estimates of cell number in the MNTB and LSO at 3 months, and for MSO at 30 months (which represent the maximum estimates across their study), are lower than those reported by most other groups, including ourselves. The reports of Irving and Harrison (1967) and Okoyama et al., (1995), also suggest that fewer neurons are found in the principal nuclei of the SOC than our data indicate. In contrast, two other reports (Reuss et al., 1999; Reimann and Reuss, 1999) indicate that considerably more neurons are located in the MNTB and MSO than we counted in this study, and their estimate of nearly 8,000 LSO cells is more than three times our estimate. These discrepancies may be due, at least in part, to the manner in which certain SOC structures were delineated. Reimann's group adopted the nuclear borders depicted in the Paxinos and Watson (1986) stereotaxic atlas of the rat brain, whose parcellation of the SOC differs considerably from ours, particularly at rostral and caudal levels. Although this atlas has demonstrated its usefulness as a nearly universal stereotaxic reference, we relied on more specialized sources of information and applied cytological and morphological criteria for accurate delineation of nuclear boundaries.

Counts of SPON neurons have appeared in several previous reports (Reimann and Reuss, 1999; Reuss et al., 1999; Kulesza and Berrebi, 2000; Saldaña and Berrebi, 2000), and the resulting estimates are quite consistent across studies. Regarding other periolivary nuclei, we cannot easily compare our LNTB and VNTB counts with the data of Reimann and Reuss (1999) or Reuss et al. (1999) because these authors parcelled this territory into a ventral periolivary region (VPO; which presumably corresponds to both the VTNB and LNTB) and a dorsal periolivary region (DPO). In any event, their estimate of 1,100 neurons in the VPO cannot be reconciled with our count of more than 6,600 cells in the VNTB and LNTB combined. We offer the possibility that a significant number of neurons in the rostral and caudal extremes of the SOC, which according to our data are dominated by VNTB and LNTB, were included in their LSO counts. Interestingly, the combined counts for LSO, LNTB and VNTB reported in our study (9,100 neurons) compares favorably with the combined estimates for DPO, VPO and LSO in the reports published in 1999 by Reimann and Reuss (10,100 neurons) and Reuss et al., (9,835 neurons). 
We are aware of one study in which the rat DNLL was reported to contain 9,000 neurons (Bajo et al., 1993), but not enough procedural information was provided to determine how this estimate was obtained.

Clearly, variability between studies in counting methods and in the criteria used to determine nuclear borders represents a major source of the discrepancies in neuronal estimates. However, other factors, such as the genetic strain or gender, which are known to affect the growth rate of experimental animals (Svendsen and Hau, 1994; Klinke, 2000), may have also contributed to the disparities noted. For example, recent data provided by a commercial supplier of laboratory rats indicate considerable variability in the growth rates of different background strains (www.m-b.dk/Growthrates/out_inbred_rats.htm\#SPRD). Female Sprague-Dawley rats at 12 weeks of age weigh $260 \mathrm{~g}$ and males weigh $432 \mathrm{~g}$, while Wistar rats of comparable age weigh $202 \mathrm{~g}$ [females] and $321 \mathrm{~g}$ [males]. It is known that, regardless of the strain, laboratory rats of comparable weight tend to have brains of similar size (Paxinos et al., 1985), but it remains to be ascertained whether differences in body weight are correlated with the number of neurons found in the brain. Therefore, systematic studies with unbiased counting procedures are needed to determine how neuron number changes throughout the animal's lifespan. Until such information is made available, inter-animal variability due to strain, gender and body weight remains an important factor to consider when comparing across studies.

\section{Convergence and divergence in the auditory pathway}

The IC is a synaptic target of virtually all ascending projections from lower auditory centers, and as such is considered a significant site of convergence in the auditory system. However, our data seem to challenge this view. According to our estimates the IC, with its 375,000 neurons, contains more than five times the number of neurons in all subcollicular auditory nuclei combined. Thus, it appears that divergence, rather than convergence, may be a common feature of ascending projections to the IC. Moreover, our estimates stress the relevance of neural processing that takes place within the IC by virtue of its intrinsic and commissural connections. Interestingly, our data suggest that significant convergence of inputs likely occurs 
within the MGB of the thalamus, which represents the main target of IC projections but contains only about $20 \%$ of the number of collicular neurons.

\section{Sources of GABAergic projections to the Inferior Colliculus}

It is well established that GABAergic synaptic inputs to the IC serve to modulate the spectral and temporal response properties of IC neurons (Yang et al. 1992; Park and Pollak, 1993; LeBeau et al. 1996; Koch and Grothe 1998). Our neuronal estimates also appear to expose a widely held misconception concerning the relative abundance of GABAergic projections to the IC arising from various subcollicular nuclei. Specifically, the DNLL, whose neurons are almost without exception GABAergic (Adams and Mugnaini, 1984; Zhang et al., 1998), is commonly regarded as the main source of GABAergic inhibition to the IC; however, by our count this nucleus contains only about 1,800 neurons. In contrast, the VNLL contains nearly 14,000 neurons, all of which reportedly innervate the IC (Merchán and Berbel, 1996) and two-thirds of which are GABAergic (Riquelme et al., 2001). Therefore, the VNLL is, by far, the largest source of ascending GABAergic projections to the IC. The relative contribution of the SPON, which contains approximately 2,400 GABAergic neurons that innervate the IC (Saldaña and Berrebi, 2000; Kulesza and Berrebi, 2000), is at least comparable to that of the DNLL and should not be overlooked. It is also noteworthy that most, if not all, IC neurons possess localcircuit connections (Oliver et al., 1991) and at least 15\% of them are presumably GABAergic (Mugnaini and Oertel, 1985). This suggests that despite the multiple origins of their GABAergic inputs, IC neurons themselves are likely the most abundant source of GABAergic synapses within the IC. 


\section{Acknowledgements}

The authors gratefully acknowledge the pre-submission critiques of the manuscript provided by Drs. George Spirou and Aric Agmon. This work was supported by research grant DC02266 to A.S.B. from the National Institute on Deafness and Other Communication Disorders, National Institutes of Health, and by grants BFI2000-1358 and CyL SA 079/01 to E. Saldaña. R. Kulesza was supported by a graduate teaching assistantship from the West Virginia University Department of Neurobiology and Anatomy. A. Viñuela was supported by a graduate fellowship from the Spanish Fondo de Investigación Sanitaria (FIS). The stereological counts were obtained using equipment located in the Image Analysis Facility of the West Virginia University Department of Neurobiology and Anatomy, and provided by a grant from the West Virginia University Research Corporation. 

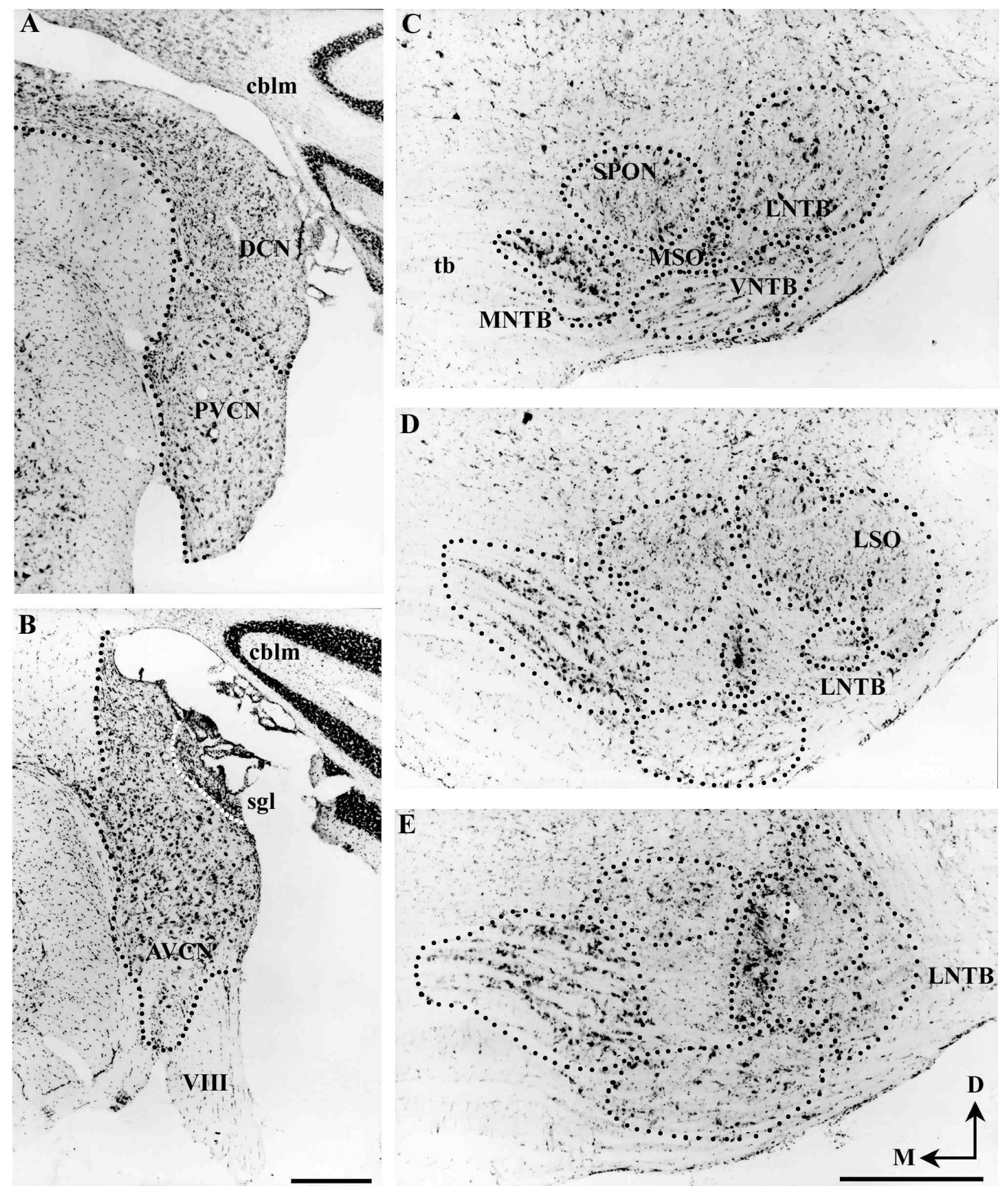
Figure 3.1. Cochlear nuclei and superior olivary complex viewed in coronal sections. A) Caudal level of the $\mathrm{CN}$ containing the DCN and the PVCN. B) More rostral level of the $\mathrm{CN}$ depicts the caudal aspect of the AVCN at the level of the vestibulocochlear nerve (VIII). A small portion of the superficial granule cell layer ( $\mathrm{sgl}$ ) is present at this level. C) This caudal section of the SOC contains the MTNB, VNTB, LNTB, MSO and SPON. Note the position of the LNTB at this level. D) At a mid rostral-caudal level of the SOC, the LSO is now prominent and shows its typical ' $\mathrm{S}$ ' shape. At this level, the LNTB is constricted and occupies a small territory underneath LSO. In the most rostral section (E), the LSO is absent and the LNTB is expanded dorsally and shifted laterally. cblm, cerebellum; D, dorsal; M, medial; tb, trapezoid body. Both scale bars $=500 \mu \mathrm{m}$ 

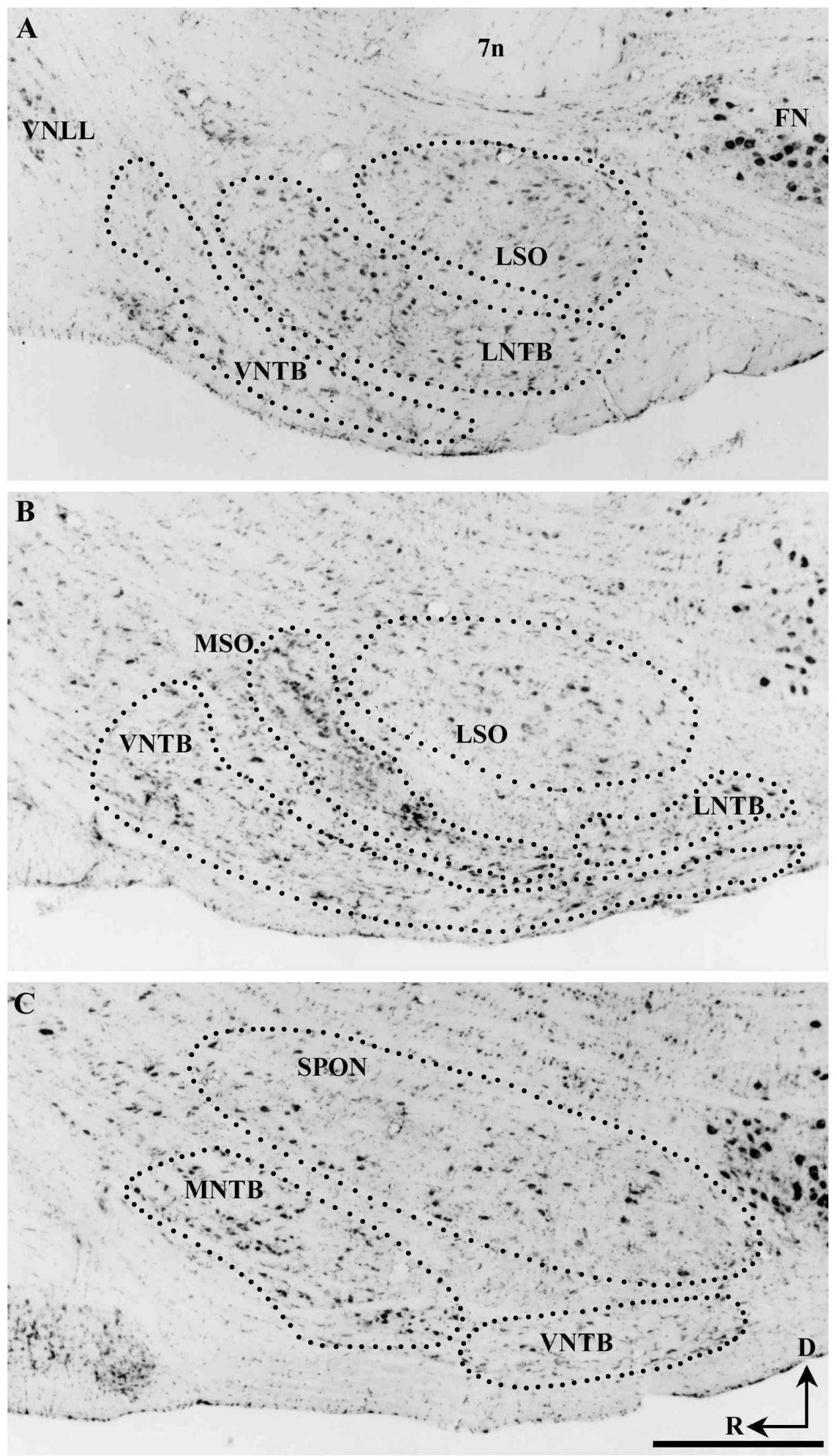
Figure 3.2. Borders of SOC nuclei in parasagittal sections. A parasagittal series of sections shows the SOC at lateral (A), middle (B) and medial (C) levels. Note the rostral extension of the LNTB and VNTB and compare to Figure 1E. 7n, facial nerve, FN, facial nucleus, R, rostral. Scale bar $=500 \mu \mathrm{m}$ 

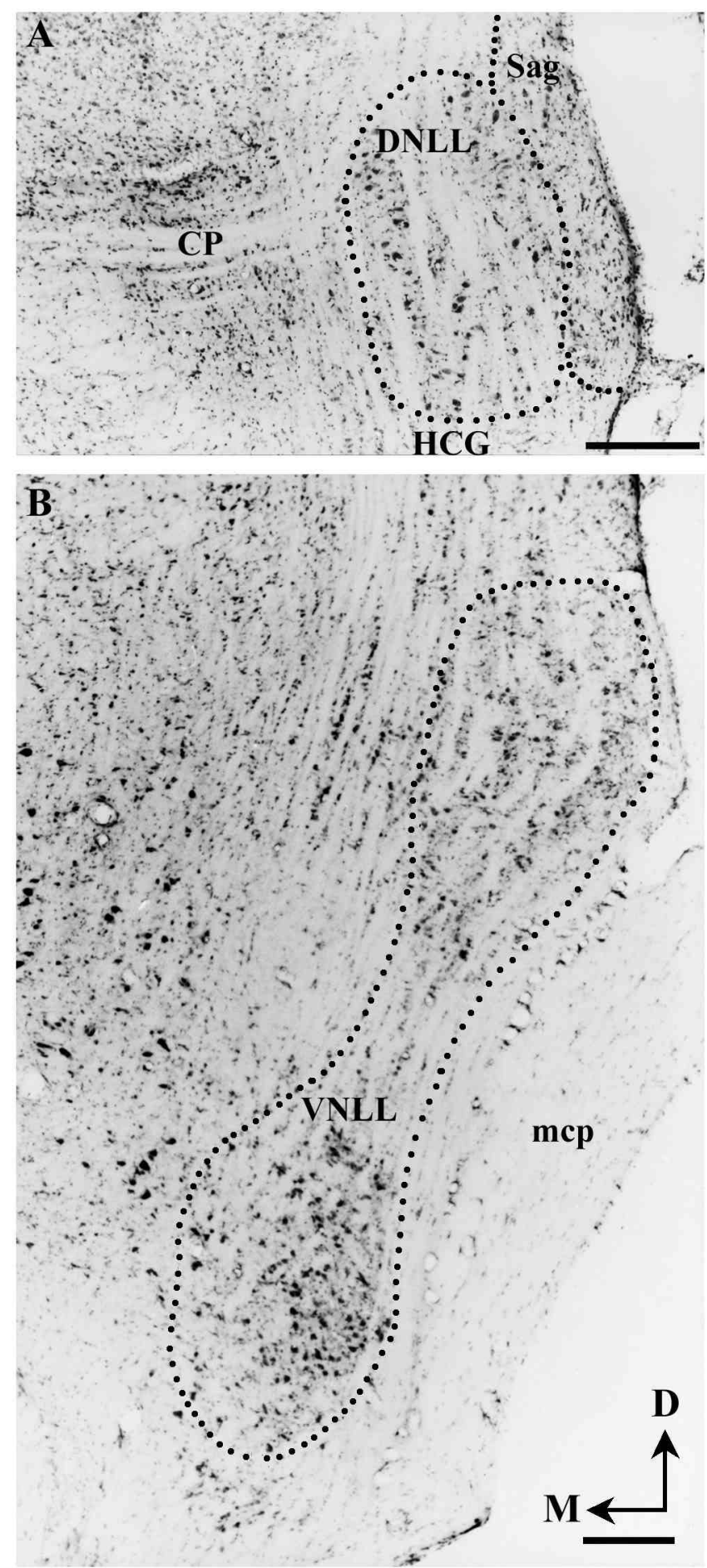
Figure 3.3. Coronal sections through the nuclei of the lateral lemniscus. (A) The DNLL is flanked by the nucleus sagulum laterally and the HCG ventrally. (B) The VNLL, which in most sections appears as an unbroken and long column oriented more or les vertically, appears at this caudal level as two separate clusters of cells. CP, commissure of Probst; mcp, middle cerebellar peduncle. Scale bar $=250 \mu \mathrm{m}$ 

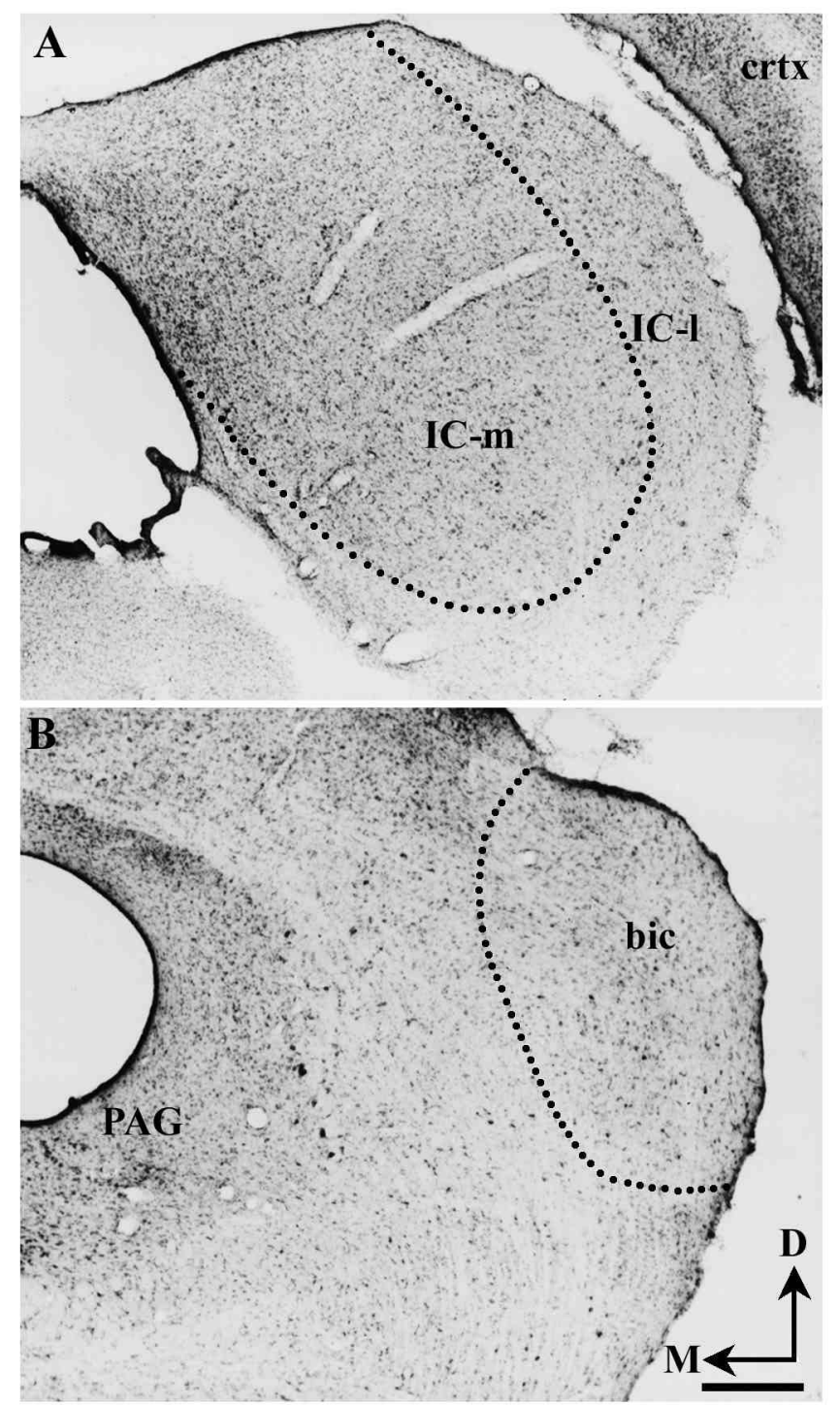

Figure 3.4. Subdivisions of the inferior colliculus in coronal section. A) The IC consists of medial (IC-m) and lateral (IC-1) subdivisions. The IC-m includes the classical dorsal cortex and central nucleus, and the lateral IC corresponds to the classical external cortex. B) A more rostral section through the midbrain tectum showing the brachium of the IC (bic). crtx, cortex; PAG, periaqueductal gray. Scale bar $=500 \mu \mathrm{m}$ 


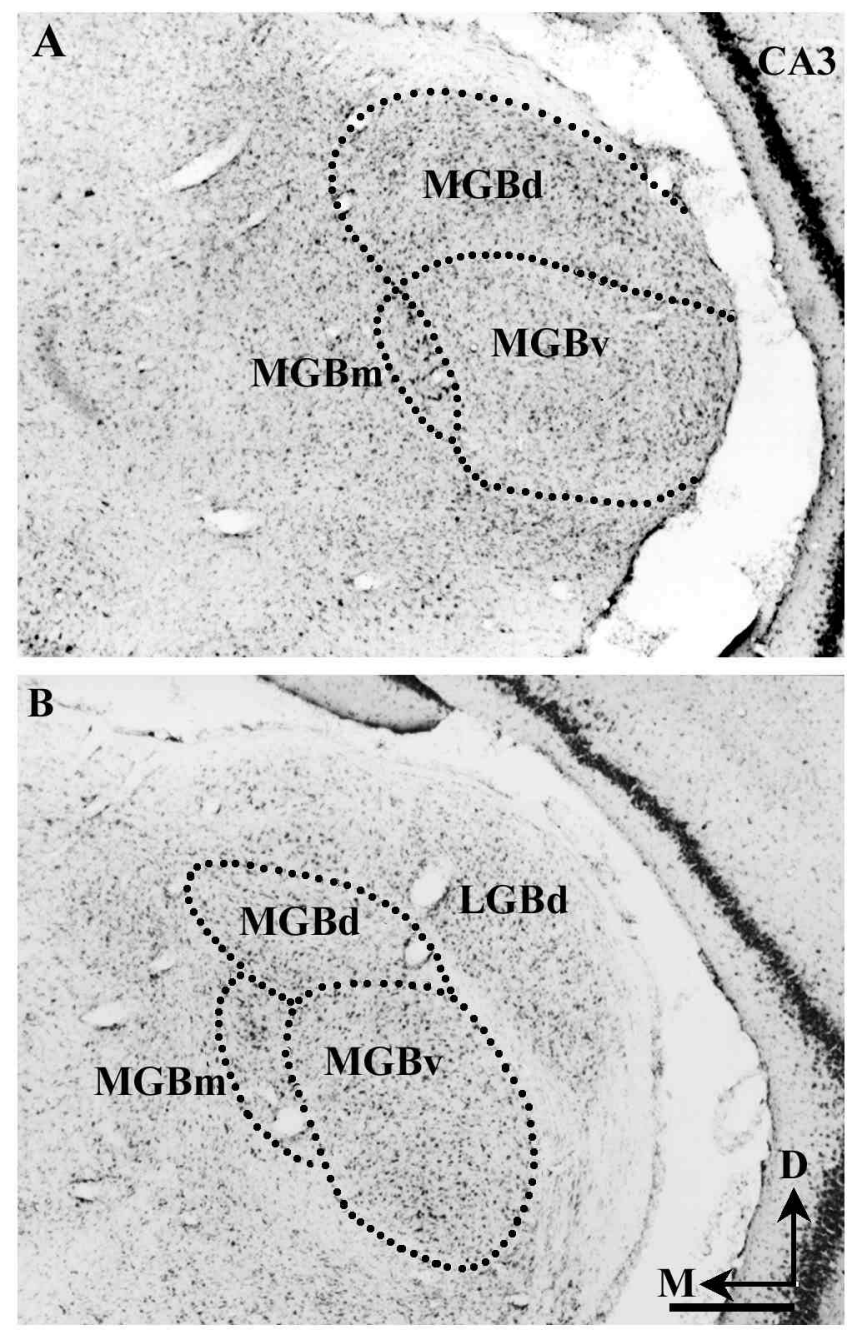

Figure 3.5. Subdivisions of the MGB. The three subdivisions of the MGB (ventral [MGBv], dorsal $[\mathrm{MGBd}]$ and medial $[\mathrm{MGBm}])$ are depicted in coronal sections at caudal (A) and rostral (B) levels. Note the medial shift in the position of the cell groups caused by the dorsal lateral geniculate body (LGBd) rostrally. CA3, area CA3 of hippocampus. Scale bar $=500 \mu \mathrm{m}$. 


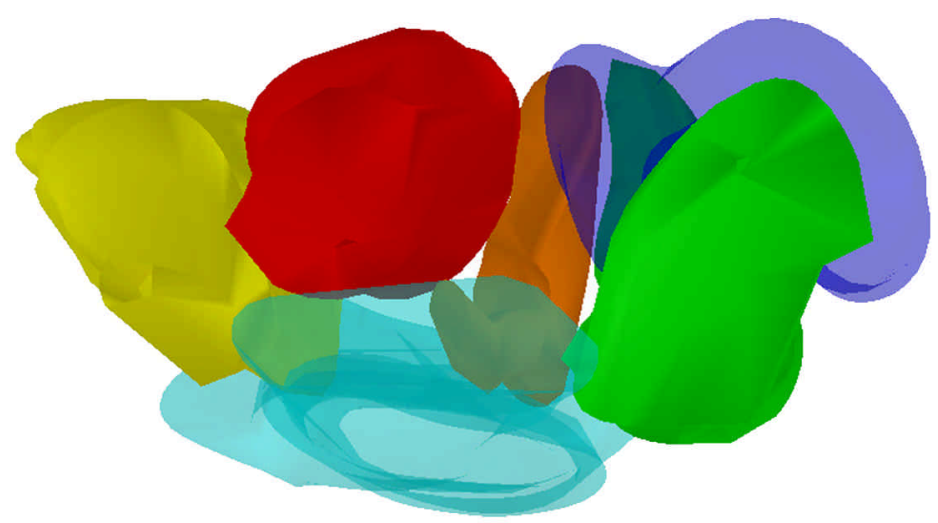

Figure 3.6. Three-dimensional reconstructions of SOC nuclei. This caudal view of the SOC has been rotated slightly medially and dorsally to provide the clearest view of all nuclei. The MNTB is yellow, the SPON red, the VNTB is a semi-transparent light blue, the MSO is orange, the LSO dark blue and the LNTB green. Note the dorsal expansion of the MSO as the nucleus courses rostrally and the manner in which the LNTB encapsulates the LSO. 
Table 3.1

Unbiased stereological estimates of the numbers of neurons $\left(\mathbf{N}_{\text {est }}\right)$ found in sub-cortical auditory structures in the rat. Mean values have been rounded to the nearest hundred.

\begin{tabular}{|c|c|c|}
\hline Structure & Abbreviation & $\mathbf{N}_{\text {est }} \pm$ S.E.M ${ }^{*}$ \\
\hline \multicolumn{3}{|l|}{ Cochlear nuclei (excluding granule cells) } \\
\hline Anteroventral cochlear nucleus & $\mathrm{AVCN}$ & $12,800 \pm 460$ \\
\hline Posteroventral cochlear nucleus & PVCN & $8,500 \pm 77$ \\
\hline \multirow[t]{2}{*}{ Dorsal cochlear nucleus } & $\mathrm{DCN}$ & $9,000 \pm 251$ \\
\hline & Total & 30,300 \\
\hline \multicolumn{3}{|l|}{ Superior olivary complex } \\
\hline Medial nucleus of the trapezoid body & MNTB & $6,000 \pm 141$ \\
\hline Medial superior olive & MSO & $1,100 \pm 18$ \\
\hline Lateral superior olive & LSO & $2,500 \pm 41$ \\
\hline Superior paraolivary nucleus & SPON & $2,400 \pm 46$ \\
\hline Ventral nucleus of the trapezoid body & VNTB & $4,500 \pm 224$ \\
\hline \multirow[t]{2}{*}{ Lateral nucleus of the trapezoid body } & LNTB & $2,100 \pm 108$ \\
\hline & Total & 18,600 \\
\hline \multicolumn{3}{|l|}{ Lateral lemniscus } \\
\hline Ventral nucleus of the lateral lemniscus & VNLL & $13,800 \pm 210$ \\
\hline Horizontal cell group & $\mathrm{HCG}$ & $600 \pm 6$ \\
\hline Dorsal nucleus of the lateral lemniscus & DNLL & $1,800 \pm 53$ \\
\hline \multirow[t]{2}{*}{ Sagulum } & Sag & $1,300 \pm 25$ \\
\hline & Total & 17,500 \\
\hline \multicolumn{3}{|l|}{ Inferior colliculus } \\
\hline Medial inferior colliculus & $\mathrm{IC}-\mathrm{m}$ & $227,000 \pm 1,958$ \\
\hline Lateral inferior colliculus & IC-1 & $128,800 \pm 3,943$ \\
\hline \multirow[t]{2}{*}{ Brachium of the inferior colliculus } & bic & $17,800 \pm 556$ \\
\hline & Total & 373,600 \\
\hline \multicolumn{3}{|l|}{ Medial geniculate body } \\
\hline Ventral medial geniculate body & MGBv & $47,500 \pm 777$ \\
\hline Dorsal medial geniculate body & MGBd & $18,700 \pm 730$ \\
\hline Medial medial geniculate body & MGBm & $6,100 \pm 126$ \\
\hline
\end{tabular}

*for systematic random samples, the standard error of the mean (S.E.M.) is calculated as the standard deviation of the mean (S.D.)/ n (Gundersen and Jensen, 1987). 


\section{Table 3.2}

Comparison of present results with previously published estimates of the numbers of neurons in central auditory structures of the rat.

\begin{tabular}{|c|c|c|c|c|c|c|c|}
\hline \multirow[b]{2}{*}{$\begin{array}{l}\text { Study/Staining method } \\
\text { (strain/gender/age-weight) }\end{array}$} & \multicolumn{2}{|c|}{$\begin{array}{c}\text { Cochlear } \\
\text { nuclei* }\end{array}$} & \multicolumn{4}{|c|}{ Superior olivary complex } & \multirow{2}{*}{$\begin{array}{c}\text { Lemniscal } \\
\text { nuclei } \\
\text { DNLL }\end{array}$} \\
\hline & $\mathrm{DCN}$ & $\mathrm{VCN}$ & MNTB & MSO & LSO & SPON & \\
\hline $\begin{array}{l}\text { Present study }{ }^{+} \\
\text {Cresyl Violet } \\
\text { (Wistar/female/190-210 g) }\end{array}$ & 9,000 & 21,300 & 6,000 & 1,100 & 2,500 & 2,400 & 1,800 \\
\hline $\begin{array}{l}\text { Agar et al., } 1999^{++} \\
\text {Cresyl Violet } \\
\text { (Wistar/male/adult) }\end{array}$ & 10,560 & 15,340 & & & & & \\
\hline $\begin{array}{l}\text { Casey, } 1990 \\
\text { Cresyl Violet } \\
\text { (Fischer/male/-) } \\
\end{array}$ & & & 2,712 & 373 & 1,430 & & \\
\hline $\begin{array}{l}\text { Irving and Harrison, } 1967 \\
\text { Silver impregnation } \\
\text { (Albino/-/adult) }\end{array}$ & & & 3,870 & 690 & 1,480 & & \\
\hline $\begin{array}{l}\text { Okoyama et al., } 1995 \\
\text { Cresyl Violet } \\
\text { (Sprague-Dawley/-/various) }\end{array}$ & & & & $690-760$ & & & \\
\hline $\begin{array}{l}\text { Riemann \& Reuss, } 1999 \\
\text { Tract-tracing } \\
(\text { Sprague-Dawley/male/200-220 g) }\end{array}$ & & & 7,432 & 1,731 & 7,780 & 2,025 & \\
\hline $\begin{array}{l}\text { Reuss et al., } 1999 \\
\text { Cresyl Violet } \\
\text { (Sprague-Dawley/male/200-220 g) }\end{array}$ & & & 7,230 & 1,610 & 7,980 & 2,240 & \\
\hline $\begin{array}{l}\text { Kulesza and Berrebi, } 2000^{+} \\
\text {Cresyl Violet } \\
\text { (Sprague-Dawley/female/230-285 g) }\end{array}$ & & & & & & 2,555 & \\
\hline $\begin{array}{l}\text { Saldaña and Berrebi, } 2000^{+} \\
\text {Cresyl Violet } \\
(\text { Albino/female } / 190-300 \mathrm{~g})\end{array}$ & & & & & & 2,633 & \\
\hline $\begin{array}{l}\text { Bajo et al., } 1993 \\
\text { Cresyl Violet } \\
\text { (Wistar/female/200 g) }\end{array}$ & & & & & & & 9,000 \\
\hline
\end{tabular}

* excludes cochlear nucleus granule cells

+ employed optical fractionator stereology tool

++ employed fractionator stereology tool

Where available, the genetic strain, gender and age or weight of the experimental animals are provided. 


\section{Chapter Four}

\section{Physiological Response Properties of Neurons in the Superior Paraolivary Nucleus of the Rat}

This work has been submitted for publication to the Journal of Neurophysiology 


\begin{abstract}
The superior paraolivary nucleus (SPON) is a prominent nucleus of the superior olivary complex. In rats, this nucleus is composed of a homogeneous population of GABAergic neurons that receive excitatory input from the contralateral cochlear nucleus and inhibitory input from the ipsilateral medial nucleus of the trapezoid body. SPON neurons provide a dense projection to the ipsilateral inferior colliculus, where their GABAergic boutons are capable of exerting profound modulatory influence on colliculus neurons. Despite recent interest in morphological and connectional features of SPON, little is presently known concerning the physiological response properties of this cell group or its functional role in auditory processing.
\end{abstract}

We utilized extracellular, in vivo recording methods to study responses of SPON neurons to broad band noise, pure tones and amplitude-modulated pure tone stimuli. Localization of recording sites within the SPON provides evidence for a medial (high frequency) to lateral (low frequency) tonotopic representation of best frequencies within the nucleus. The vast majority of SPON neurons have very low rates of spontaneous activity. The vast majority of SPON neurons responded only to stimuli presented in the contralateral ear; a small population showed binaural facilitation. Nearly all SPON neurons responded at the offset of broad band noise and pure tone stimuli. Best frequencies of SPON neurons spanned the audible range of the rat and receptive fields were narrow with V-shaped regions near threshold. Approximately 50\% of SPON neurons displayed duration selectivity, a feature that has previously not been described below the level of the inferior colliculus. The majority of SPON neurons also display spike activity synchronized to pure tones that are sinusoidally amplitude-modulated up to $200 \mathrm{~Hz}$. Taken together, these findings suggest that the SPON is capable of encoding temporal features of complex sounds, such as those contained in species-specific vocalizations. 


\section{INTRODUCTION}

The superior olivary complex (SOC) is a constellation of brainstem cell groups involved in auditory processing. The three principal SOC nuclei, the medial nucleus of the trapezoid body (MNTB), medial superior olive (MSO) and lateral superior olive (LSO), have well-defined roles in localization of sounds based on interaural timing and intensity difference cues. The remaining cell groups of the SOC are collectively termed periolivary nuclei, and their function in hearing is poorly understood. The superior paraolivary nucleus (SPON) is a prominent periolivary cell group in rodents that receives an excitatory input from octopus and multipolar cells in the contralateral cochlear nuclear complex (Friauf and Ostwald 1988; Thompson and Thompson 1991; Schofield and Cant 1995) and a substantial inhibitory innervation from the ipsilateral MNTB that is mediated by the neurotransmitter glycine (Banks and Smith 1992; Bledsoe et al. 1990; Helfert et al. 1989; Moore and Caspary 1983; Schofield 1994; Sommer et al. 1993). While recent reports indicate that in rats a substantial descending projection to the SPON originates in the tectocommissural column, a recently identified structure situated within the commissures of the inferior and superior colliculi (Viñeula et al. 2001), the central nucleus of the inferior colliculus does not innervate the SPON (Faye-Lund 1986; Caicedo and Herbert 1993). Projections of the rat SPON target the ipsilateral inferior colliculus and are topographically organized (Kelly et al. 1998b; Saldaña and Berrebi 2000). Given that virtually all of the estimated 2,400 SPON neurons in rats participate in this projection and utilize GABA as their neurotransmitter (Saldaña and Berrebi 2000; Kulesza and Berrebi 2000; Kulesza et al. 2002), the nucleus is poised to exert profound inhibitory influence on neurons in the inferior colliculus.

In contrast to our understanding of SPON anatomy, the physiological response properties of its neurons are not well studied. Previous recordings from the SPON in gerbils indicate a heterogeneous population of units with mixed binaural and monaural responses, wide ranging rates of spontaneous activity, and both sustained and phasic discharges (Behrend et al. 2002; Dehmel et al. 2002; Spitzer and Semple 1995). The few published recordings from the SPON of cat (Guinan 1972) and rat (Finlayson and Adams 1997) support the notion of heterogeneous responses to sounds. Thus, based on the available data obtained from different species it is difficult to discern a functional role for SPON in auditory processing. We undertook a 
systematic study of auditory evoked responses of SPON neurons in the rat. The choice of species was based, in large part, on the previously demonstrated homogeneity of neuronal morphologies, neurochemical phenotypes, and efferent projections of SPON neurons in rats (Saldaña and Berrebi 2000; Kulesza and Berrebi 2000). 


\section{METHODS}

\section{Stereotaxic surgery}

This study employed 40 female albino rats (Sprague-Dawley strain) and 5 female hooded rats (Long Evans strain) weighing between 250 and 320 grams. Animals were anesthetized by intramuscular injection of a mixture of xylazine and ketamine $(8.6 \mathrm{mg}$ and $57 \mathrm{mg}$ per $\mathrm{kg}$ body weight, respectively). Once determined to be areflexic, the rats were placed into a stereotaxic frame, their heads secured by a bite bar, and custom-made hollow brass earbars inserted into the cartilaginous external auditory meatus. A midline incision was then made in the scalp, a small bone flap overlying the cerebellum was removed, and the dura matter was incised to permit penetration of the electrode. The anesthetic state of each animal was monitored throughout the experiment and supplemental doses of the same anesthetics were given, as needed, at 2/5 the original dose.

\section{Sound stimuli and delivery}

Acoustic stimuli were delivered via Stax speakers contained within custom built housings (Sokolich, 1981) attached to the hollow ear bars. To permit calibration of the sound delivery system, the hollow ear bars were machined with a small calibration tube joining the sound delivery tube at a $45^{\circ}$ angle. Prior to or immediately following each experiment, a B \& $\mathrm{K}$ microphone was placed into the calibration tube and the sound delivery system calibrated for broad band noise $(\mathrm{BBN})$ and pure tones between 1 and $40 \mathrm{kHz}$. Stimulus intensities were converted to dB SPL offline.

Sound stimuli were created digitally with SigGen or RP Visual Design Studio software (Tucker-Davis Technologies, Gainesville, FL), and had $5 \mathrm{~ms} \cos ^{2}$ ramps. Single- unit data was collected using Brainware software (Tucker-Davis Technologies, Gainsville, FL) and analyzed using Microsoft Excel and custom written Matlab scripts.

\section{Physiological Recordings}

Both tungsten and glass micropipette electrodes $(8-20 \mathrm{M} \Omega$, filled with $3 \mathrm{M} \mathrm{KCl}$ and $2.5 \%$ 
biocytin) were used to record from 116 units in the SPON. Electrodes were advanced into the SPON from a dorsal approach with a Burleigh Inchworm (Burleigh Instruments, Inc., Victor, NY) and using stereotaxic coordinates obtained from the atlas of Paxinos and Watson (1986). The data obtained with tungsten and glass electrodes are identical and will therefore be considered together. Although not included in this report, units recorded with glass electrodes filled with $0.45 \% \mathrm{NaCl}$ also yielded similar results. Recording sites were marked with lesions using tungsten electrodes ( $8 \mu \mathrm{A}$ for 10 seconds) or deposits of biocytin (Sigma Chemical Co., St. Louis, MO) using glass micropipettes (200 nA for 5 minutes, $50 \%$ duty cycle).

Binaural BBN (50 msec in duration, $20 \mathrm{~dB}$ attenuation) was used as a search stimulus. Responses were determined to be from a single unit if they had biphasic waveforms and constant amplitude peaks. Upon isolation of a single unit, its aurality was determined by examining responses to twenty repetitions each of binaural, ipsilateral and contralateral BBN. Neurons were considered monaural if their firing rate in response to unilateral stimulation did not differ significantly from their response to binaural stimulation, and considered to be binaurally facilitated if they displayed significantly more spikes in response to the binaural presentation.

Response maps were generated from unit responses to presentations of numerous frequency-intensity combinations of pure tones (each $50 \mathrm{~ms}$ in duration). Best frequency (BF), the frequency that elicited a response from the unit at the lowest sound intensity, was determined from the response map. Threshold was defined as the lowest intensity that elicited a response from the unit. Rate-level curves were recorded from 55 units. Peri-stimulus time histograms (PSTHs) were generated from 500 presentations of $50 \mathrm{~ms}$ BF tones at $20 \mathrm{~dB}$ above threshold. Spontaneous activity was monitored in a $10 \mathrm{~ms}$ time window before stimulus presentation during each of 500 sweeps, for total time of 5 seconds.

A subset of SPON units $(n=22)$ were presented with twenty repetitions of their BF tone (20dB above threshold) at varying durations (10, 25, 50, 100 and $200 \mathrm{~ms}$ ). If the spike count for a particular duration was $50 \%$ greater than the spike counts for longer or shorter durations, the unit was considered duration sensitive (Ehrlich et al. 1997). A partially overlapping population of cells was presented with sinusoidally amplitude-modulated (SAM) pure tones (100\% 
modulation, $\mathrm{n}=30$ ). Each stimulus was presented 20 times and the carrier frequency was a 500 $\mathrm{ms} \mathrm{BF}$ tone (20 dB above threshold) coupled with modulation frequencies of 50, 100, 200, 300 or $400 \mathrm{~Hz}$. Fidelity of phase locking was determined by calculating vectors strengths (VS; Goldberg and Brown 1969) at each modulation frequency using the equation:

$$
\mathrm{VS}=\sqrt{\left[\sum \sin \left(a_{i}\right)\right]+\left[\sum \cos \left(a_{i}\right)\right]^{2}} / N
$$

where $a_{i}$ is the phase of spike $i$ relative to the frequency modulation and $N$ is the total number of spikes. A vector strength of 1 indicates perfect synchrony between modulation phase and the neuronal response, while a value of 0 indicates no correlation between modulation phase and the response. The unit response at the termination of the $500 \mathrm{~ms}$ SAM tone was not considered in the calculation of vector strength.

\section{Localization of recording sites}

Upon completion of the recording session, each animal was given a supplemental dose of xylazine and ketamine and perfused through the ascending aorta with a vascular rinse of normal saline followed by a fixative composed of $4 \%$ paraformaldehyde and $0.1 \%$ glutaraldehyde in $0.12 \mathrm{M}$ sodium phosphate buffer, $\mathrm{pH}$ 7.2. The brain was then dissected from the cranium and cryoprotected overnight in $30 \%$ sucrose in the same buffer. Brainstems were coronally sectioned on a freezing microtome at a thickness of $60 \mu \mathrm{m}$. If recording sites in that animal were marked by electrolytic lesions, the tissue sections were dry mounted onto glass slides from gelatinalcohol and stained for Nissl substance with cresyl violet using standardized protocols. Sections from animals that received biocytin injections were processed, free floating, according to the ABC method (Vector Laboratories, Burlingame, CA) using 0.05\% diaminobenzidine, $0.01 \%$ hydrogen peroxide, $0.025 \%$ cobalt chloride and $0.02 \%$ nickel ammonium sulfate as the chromogen. The sections processed in this manner were then mounted onto glass slides and counterstained with neutral red.

Camera lucida drawings were made of tissue sections containing biocytin deposits, electrolytic lesions, or evidence of electrode passage. The distance between any two landmarks 
(lesions or deposits of biocytin) along a recording track was measured and used to calculate tissue shrinkage. Using depth measurements taken directly from the Burleigh microdrive readout and adjusting for tissue shrinkage, recording site locations along the electrode track were plotted by superimposing the camera lucida drawings onto standardized templates of the rat SOC (Paxinos and Watson 1986) at the appropriate rostrocaudal level and aligned to achieve the best possible fit. 


\section{RESULTS}

\section{Localization of units and tonotopic mapping of best frequencies}

To elucidate the auditory evoked responses of SPON neurons, we recorded from a total of 116 well-isolated single units from 45 rats. Each electrode track was reconstructed and the location of each unit within the SPON confirmed by biocytin deposition or lesion demarcation (Fig. 4.1).

The $\mathrm{BF}$ of each single unit was then determined to reveal the representation of frequency in the SPON and used to construct a tonotopic map of the nucleus. In our recordings, BFs ranged from $1.1 \mathrm{kHz}$ to $40 \mathrm{kHz}$, which covers the most sensitive range of hearing in rats (Kelly and Masterton 1977). Moreover, we found that the BFs of SPON units varied in a systematic fashion with the lowest BF units located laterally, units with middle BFs located in the central portion of the nucleus, and those with the highest BFs located medially (Fig. 4.2). When multiple SPON units were encountered along a vertical penetration, the BF increased with depth, suggesting the existence of curved or dorsomedially-to-ventrolaterally tilted isofrequency contours.

\section{Responses to broad band noise and pure tones}

Aurality of responses

A total of fifty-three SPON units were presented with ipsilateral, contralateral and binaural BBN. All of the neurons tested responded to the BBN stimulus (Fig. 4.3). The great majority of neurons (96\%) responded only at the BBN offset, one neuron responded only at the BBN onset (2\%) and one neuron responded at the onset and again at the offset of the BBN (2\%). Forty-six cells $(87 \%)$ responded only to contralateral stimulation and gave rise to an equivalent number of spikes in response to binaural BBN $(p>0.05$, paired $t$-test). These neurons were, therefore, classified as $0 \mathrm{E}$. In seven neurons (13\%) the binaural $\mathrm{BBN}$ presentation evoked significantly more spikes than the contralateral stimulus ( $p \leq 0.047$, paired $t$-test), indicating 
binaural facilitation. These seven neurons were classified as $0 \mathrm{E}-\mathrm{F}$, and all but one of these responded only at the $\mathrm{BBN}$ offset. None of the 53 neurons tested responded to ipsilateral stimulation alone. Spontaneous rates of activity in SPON neurons were generally very low (see below), but in the few units with measurable spontaneous activity we noted a depression in the spike rate during the contralateral BBN stimulus, suggesting the possibility of a contralaterally derived inhibitory input. However, since most neurons displayed virtually no spontaneous activity, we cannot rule absolutely out the possibility of an ipsilaterally driven inhibitory input.

\section{Response types}

Peri-stimulus time histograms (PSTHs) were constructed for all 116 SPON unit responses to $\mathrm{BF}$ pure tones at $20 \mathrm{~dB}$ above threshold (Fig. 4.4). Greater than $95 \%$ of all units were characterized by spike activity primarily at the end or "offset" of the stimulus. Distinguishing features of these offset responses, however, enabled us to further sub-divide our sample into five response classes including I) neurons that responded only transiently at the stimulus offset with single spikes (average of $1.13 \pm 0.55$ spikes per stimulus), termed "offset-transient" responders (Fig. 4.4A); II) units that responded only at the stimulus offset with two or more regularly spaced spikes (average of $1.98 \pm 0.82$ spikes per stimulus), termed “offset-choppers" (Fig. 4.4B); III) units that responded only at the stimulus offset with spike activity that was sustained for more than $20 \mathrm{~ms}$ (average of $2.01 \pm 1.07$ spikes per stimulus), termed "offset-sustained" responders (Fig. 4.4C); IV) units that responded with a few spikes during the stimulus as well as offset spikes, termed "on-offset" responders (Fig. 4.4D); and V) neurons that displayed a mixture of onset and sustained responses during the stimulus but no offset spikes, termed "on-sustained" responders. Interestingly, members of this last response class, that accounted for only $5 \%$ of the units, were all localized near the dorsomedial border of the SPON and all but one had BFs below $4 \mathrm{kHz}$. For the remaining response classes, the range of BFs was from 1.2 to $40 \mathrm{kHz}$ for offsettransient neurons, from 2.7 to $20.2 \mathrm{kHz}$ for offset choppers, from 2.8 to $11.7 \mathrm{kHz}$ for offsetsustained responders and from 3.5 to $26.9 \mathrm{kHz}$ for the on-sustained units.

PSTHs in response to contralateral BBN were constructed for 74 units and compared to the PSTHs derived in response to the BF pure tone stimuli. The majority of units (64 of 74, 
$86 \%$ ) were classified similarly for both stimulus types, and 9 of the remaining 10 neurons whose classification differed remained within offset classes I-III.

\section{Response latencies}

The first spike latency to $50 \mathrm{~ms}$ BF tones was determined for 78 SPON neurons in response classes I-III and averaged $7.04 \pm 3.56 \mathrm{~ms}$ from the stimulus offset. The response latencies of offset-transient units $(n=35 ; 6.05 \pm 2.33 \mathrm{~ms})$ and offset-chopper cells $(\mathrm{n}=25 ; 6.62 \pm$ $2.21 \mathrm{~ms})$ were significantly equivalent, $(\mathrm{p}=0.47$, unpaired $t$-test $)$, but both classes had significantly shorter first spike latencies than offset-sustained units $(n=18 ; 9.67 \pm 5.28 \mathrm{~ms}$; $\mathrm{p}<$ 0.02). Furthermore, the first-spike latency within response classes I-III did not correlate with the unit's BF ( $r=-0.127)$ but did appear to be related to the stimulus duration. Specifically, in 12 of 26 units so tested, response latency decreased with increasing duration $(25,50,100,200,500$ $\mathrm{ms}$ ) of BF pure tones, in two units the latency increased with increasing tone duration, and in the remaining twelve units, the latency remained relatively constant.

\section{Spontaneous activity}

Spontaneous rates of activity in SPON units averaged $2.65 \pm 10.42$ spikes per second. More than half of the units (71 of 116; 62\%) displayed no spontaneous activity at all, and only 6 cells (5\%) had spontaneous firing rates above 6 spikes per second (Fig. 4.5). For comparison, the spontaneous firing rates we recorded from $16 \mathrm{MNTB}$ units averaged $46.06 \pm 27.37$ spikes per second.

\section{Receptive fields}

The next step in our experimental protocol was to present pure tone stimuli over a wide range of frequencies and intensities. Response maps were constructed for 54 SPON units with BFs ranging from 2.3 to $40 \mathrm{kHz}$ (Fig. 4.6). Response maps typically had narrow V-shaped peaks near BF and often had low frequency tails. Generally, SPON units exhibited only offset spikes throughout their response maps; very seldom were spikes observed during the BF tone 
presentation, and these were observed at high intensity, low frequency combinations (Fig. 4.7).

Sharpness of tuning of SPON units was determined by calculating Q values, which provide a meaure of the width of the tuning curve relative to the neurons BF. Q10 and Q30 values averaged $6.77 \pm 3.30$ and $1.64 \pm 0.99$ respectively (Fig. 4.8A). The Q10 values observed for SPON are close to those reported for MNTB neurons in cat $(\mathrm{Q} 10=7.02$, Guinan 1968; Fig. 4.8B) and DNLL neurons in rat $(\mathrm{Q} 10=7.9$, Kelly et al. 1998). Q values were not statistically different across PSTH categories ( $\mathrm{p}>0.52$, unpaired $t$-test). There was a general trend of $\mathrm{Q}$ values increasing with $\mathrm{BF}$, but the statistical correlation was not significant $(\mathrm{Q} 10 \mathrm{r}=0.307$ and $\mathrm{Q} 30 \mathrm{r}=0.308)$.

Thresholds for SPON neurons at their BF averaged $25.41 \pm 11.53 \mathrm{~dB}$ SPL and ranged from 5 to $48 \mathrm{~dB}$ SPL (Fig. 4.9). Thresholds were not significantly different across the BF pure tone response classes $(\mathrm{p}>0.25$, unpaired $t$-test).

To determine the neuron's responsiveness to sound intensity, rate level curves were collected for 55 units and were classified as having wide dynamic ranges (greater than $20 \mathrm{~dB}$ ), narrow dynamic ranges (less than $20 \mathrm{~dB}$ ), or as being unsaturated at the highest intensity presented. Since most neurons had little or no spontaneous activity (Fig. 4.5), dynamic range was measured between the first sound intensity that generated a spike and the maximum response generated by the cell. The majority of offset-transient units (20 of 26, 77\%), cells typically firing only one spike per stimulus, had short dynamic ranges as did the majority of offset-choppers ( 6 of 7, 85\%). However, the offset-sustained $(n=15)$ and on/offset neurons $(n=6)$ were equally distributed among the three rate level classes. These data indicate that 1) most SPON neurons have short dynamic ranges; they are either unresponsive or maximally responsive within a short window of sound intensity and 2) most offset-transient and offset-chopper units are relatively unresponsive to sound intensity.

\section{Duration selectivity}

To determine whether SPON neurons displayed selectivity for stimulus duration, 22 
neurons were presented with $\mathrm{BF}$ tones of varying duration at $20 \mathrm{~dB}$ above threshold. Neurons were considered duration selective if the number of spikes evoked by any single duration, or a range of durations, was at least $50 \%$ greater than the number of spikes evoked by longer or shorter tone durations (Ehrlich et al. 1997). At all the tone durations tested, these 22 SPON units displayed responses only at the stimulus offset (Fig. 4.10A). Nine SPON units (41\%) fired more robustly at longer than at shorter durations and were classified as long duration selective (Fig. 4.10B). The BFs of these long duration selective cells ranged from 3.1 to $36 \mathrm{kHz}$. Moreover, these cells were primarily from the offset-sustained (5 of 9) and the offset-chopper (3 of 9) response classes, with one unit from the offset-transient category. One single unit tested (4\%), an offset-transient unit, fired more spikes at shorter than at longer durations and was therefore classified as short duration selective. Twelve neurons (55\%) were non-selective to stimulus duration; they fired a relatively constant number of spikes across stimulus durations. Of these, 6 were offset-transient units, 2 were offset-choppers, 2 were offset-sustained responders and 2 were on-offset units.

\section{Responses to amplitude modulations}

To examine the responses of SPON units to more complex sounds, sinusoidally amplitude-modulated (SAM) pure tones were presented to 30 units. The majority (25 of 30, $83 \%$ ) of neurons tested responded to the envelope of SAM stimuli as if each wave of modulation were being detected as a separate stimulus, and discharged to each modulation with single offset spikes (Fig. 4.11). Within this group, 14 units were offset-transients, 7 were offset-sustained responders, 3 were offset-choppers and one was an on-offset unit. Regardless of the modulation frequency, the units displayed an offset response to each presentation of the $500 \mathrm{~ms}$ SAM stimulus. BFs for the units responding to SAM stimuli ranged from 1.2 to $35 \mathrm{kHz}$ and all 25 cells responded at modulations up to $100 \mathrm{~Hz}$, with an average vector strength of $0.81 \pm 0.02$, demonstrating high fidelity phase-locking. At the lower modulation frequencies $(25-100 \mathrm{~Hz})$, SPON units followed the stimulus envelope faithfully, as indicated by vector strengths greater than VS $=0.75$. However, at the higher modulation frequencies (200 to $400 \mathrm{~Hz}$ ) vector strengths decreased as discharge rates declined sharply. However, as shown in the modulation transfer function (Fig. 4.12), even at modulation rates of 200 and $400 \mathrm{~Hz}$, the few remaining spikes were 
still phase-locked. The neurons that did not respond to SAM stimuli had BFs that ranged from 6.6 to $17.1 \mathrm{kHz}$ and fell into all PSTH response classes (offset-transient, offset-chopper, offsetsustained and on-offset). 


\section{DISCUSSION}

This study represents the first systematic characterization of the auditory evoked physiological responses of SPON neurons in the rat. Our sample of 116 single units was nearly exclusively monaurally activated, and always by contralaterally presented stimuli. SPON units responded at the offset of pure tone stimuli with a first spike latency on the order of 6-10 ms from the stimulus offset. Furthermore, many SPON units displayed stimulus duration selectivity and impressive phase-locking to amplitude modulated tones. The homogeneity of responses observed in this study is consistent with the morphological, connectional and neurochemical homogeneity previously reported for SPON neurons in the rat (Saldaña and Berrebi, 2000; Kulesza and Berrebi, 2001). In light of reports indicating considerable variability and interspecies diversity in the anatomical and physiological features of SPON neurons in other species (Moore and Goldberg 1966; Guinan et al. 1972; Osen et al. 1984; Strutz and Spatz 1980; Adams 1983; Covey et al. 1984; Saint Marie and Baker 1990; Schofield 1991; Spangler and Warr 1991; Thompson and Thompson 1991; Spitzer and Semple 1995; Behrend et al. 2002; Dehmel et al. 2002; Saldana and Berrebi 2000; Kulesza and Berrebi 2000), we suggest that future attempts to understand the functional role of SPON-derived inhibition to the IC may be most efficiently undertaken in rats.

\section{Previous studies of SPON physiology}

Kuwada and Batra (1999) described a population of neurons in the SOC of the unanesthetized rabbit that exhibited offset responses and phase locked to SAM stimuli. Although the nature of their chronic recording preparation precluded precise histological localization of recording sites, these units were located medial to the MSO and likely represent neurons of the SPON or its homologue in the rabbit. In the mustached bat, the MSO contains a population of GABAergic neurons (Winer et al. 1995) and units that demonstrate offset responses to contralateral stimulation but do not respond to ipsilateral stimulation, and have very low rates of spontaneous activity and low-pass filter characteristics for amplitude modulated tones (Grothe 1994). These findings have lead to the speculation that neurons which constitute 
the SPON in rodents may have merged into the MSO in the mustached bat (Grothe et al. 1992, Vater, 1995).

Finlayson and Adams (1997) recorded from auditory brainstem neurons of Long-Evans hooded rats anesthetized with a mixture of pentobarbital, xylazine and ketamine. They reported that SPON units were binaurally excited ("EE") and received matching BF inputs from the two ears. In the course of the present study, we recorded from more than 250 neurons localized to the SOC and reticular formation, but that were clearly not contained within SPON. Only 21 units from within this sample had "EE" properties and 13 of these were localized to the reticular formation. It is not possible to fully reconcile this dramatic discrepancy, although differences in anesthetic agents employed is one obvious factor that may have contributed to the disparate results. Furthermore, while we cannot exclude the possibility that there are substantial numbers of "EE" neurons in the rat SPON that we were unable to isolate, it is more likely, in our opinion, that at least some of the binaurally excited neurons described by Finlayson and Adams were located outside the SPON,

Recently, two reports of physiological responses of SPON neurons in gerbil have appeared in the literature and the results of these two studies are somewhat contradictory (Behrend et al., 2002; Dehmel et al., 2002). For example, Dehmel and colleagues indicate that nearly $65 \%$ of SPON neurons responded at the stimulus offset, while Behrend and co-workers reported that only $6 \%$ of their units displayed offset responses. Dehmel and co-workers did not utilize SAM stimuli in their protocol, but Behrend et al. indicates that the small population of “off” responders they identified were not capable of phase-locking at all. This finding is difficult to reconcile with our demonstration that offset neurons in the rat SPON phase-lock exceptionally well, at least to modulation frequencies up to $200 \mathrm{~Hz}$. One significant difference between the SPON of gerbils and rats is the existence in the former of a population of neurons with descending projections to the cochlear nuclei (Helfert et al. 1988' Faye-Lund 1986; our unpublished observations). Therefore, we suspect that the majority population of sustained SPON neurons recorded by Behrend et al. (2002) may serve a functional role in the descending auditory pathway that is simply not performed by the rat SPON. 


\section{Tonotopy in the SPON}

We identified a tonotopic organization within the rat SPON, with high BFs represented medially and low BFs laterally. There also appeared to be a slight dorso-medial to ventro-lateral tilt to the isofrequency contours, which was previously suggested on the basis of retrograde tracing studies (Saldaña and Berrebi 2000). Overall, the tonotopic mapping we report is consistent with the topographic arrangement of inputs to SPON from the ipsilateral MNTB (Banks and Smith 1992; Sommer et al. 1993) and the projections from SPON to the ipsilateral inferior colliculus (Kelly et al. 1998; Saldaña and Berrebi 2000). Thus, the SPON joins the principal nuclei of the SOC in having a defined tonotopic axis

\section{Duration sensitivity}

Duration is a biologically important feature of natural sounds. Indeed, neurons with selectivity for certain sound durations have been described in the inferior colliculus (IC) of bats (Casseday et al. 1994), frogs (Feng et al. 1990), and mice (Brand et al. 2000). In their recent model of duration tuning, Casseday and coworkers (2000) propose an important role for late arriving or offset inhibition. The fact that duration tuning in IC neurons can be abolished by application of bicuculline indicates that GABAergic inhibition in particular is a crucial element in the synaptic mechanisms underlying duration tuning. SPON neurons of the rat are known to utilize GABA as their neurotransmitter and they provide an impressive projection to the IC (Kulesza and Berrebi 2000; Saldaña and Berrebi 2000). It is possible, therefore, that SPON may contribute to the generation of this collicular response property. Our observation that nearly half of SPON neurons were duration selective is, to our knowledge, the first demonstration of this type of response selectivity below the level of the IC (Cassesday et al. 1994). A recent study of the mouse IC indicates that $55 \%$ of all neurons are duration tuned, including all IC neurons that display offset responses, with approximately $70 \%$ displaying long duration sensitivity (Brand et al., 2000). It would be interesting to know whether duration selective neurons in the SPON project preferentially to duration tuned neurons in the IC, but the diffuse distribution of duration sensitive neurons in both structures makes this a difficult question to address (Ehrlich et al., 1997). Nonetheless, even SPON neurons that are not duration selective may play a role in 
duration tuning of their synaptic targets in the IC by signaling the end of an acoustic stimulus.

\section{Amplitude modulations}

Another interesting feature of SPON physiology is that nearly all of its constituent neurons respond to SAM tones by phase-locking to the end of each modulation cycle. SPON neurons follow low modulation frequencies $(25-100 \mathrm{~Hz})$ with high fidelity. However, at higher frequency modulation SPON neurons still displaying high vector strengths but fail to respond to every modulation cycle, so that response rate during the stimulus falls off considerably. At the highest frequency modulation tested $(200-400 \mathrm{~Hz})$, many units only responded once at the offset of the 500 ms SAM stimulus.

We suggest that the reduced phase-locking to the higher modulation frequencies was caused by the glycine-mediated inhibition of SPON units that originates in the ipsilateral MNTB. Indirect support for this notion comes from comparing SAM responses we recorded from MNTB cells with those of SPON neurons. Figure 4.13 depicts typical responses to $50 \mathrm{~Hz}$ SAM stimulus for MNTB and SPON units with similar BFs. MNTB neurons fired robustly during the stimulus, with their PSTH pattern apparently carved out by the modulation frequency. Only during the short period between each wave of modulation were the MNTB neurons quiescent for a few milliseconds. Interestingly, it is during this same phase of the modulation cycle, corresponding to the trough of the SAM stimulus waveform, that SPON neurons discharged their action potentials. This reciprocal temporal firing pattern suggests that SPON units are capable of discharging only when released from their MNTB-derived inhibition, suggesting a inhibitory rebound mechanism. Along the same line of reasoning it is plausible that during stimulation at high modulation rates SPON units may have insufficient time to recover from this inhibition, and thus can respond only at the termination of the SAM stimulus. An alternative explaination might be that the inhibition reaching the SPON during high frequency amplitude modulations (200 $400 \mathrm{~Hz}$ ) may not be long enough to cause an inhibitory rebound during each modulation cycle. Other known sources of input to the SPON, in particular cells located in the posteroventral cochlear nucleus with onset PSTHs that presumably correspond to excitatory octopus neurons, reportedly have superior phase-locking capabilities to SAM stimuli (Frisina et al. 1990). 
However, it is unclear at this point how input from octopus cells influences SPON neurons during SAM stimulation.

Through the course of our experiments, we managed to record data from a population of MNTB units. MNTB neurons responded to SAM stimuli in a sustained manner; at low modulation frequencies $(50 \mathrm{~Hz})$ PSTHs from MNTB neurons had peaks and valleys that corresponded to the stimulus waveform. At higher modulation frequencies $(200-400 \mathrm{~Hz})$, MNTB neurons responded in a sustained manner with no correlation to the stimulus envelope (figure 4.14). Interestingly, MNTB neurons discharged with nearly the same number of spikes to $50 \mathrm{~Hz}$ and $400 \mathrm{~Hz}$ stimuli (45.6 \pm 17.89 and $40.75 \pm 18.10$ spikes per stimulus respectively; figure 4.14). Additionally, vector strengths for MNTB were relatively low across all frequencies tested and averaged 0.18 at $100 \mathrm{~Hz}$. This is in stark contrast to the 0.81 vector strength observed in the SPON under similar conditions $(100 \mathrm{~Hz})$. A comparision of the SPON and MNTB SAM data raise some interesting points. First, the MNTB-SPON circuit provides an example where a poorly timed input (MNTB), relative to the SAM waveform, can form a temporally precise response in another neuron through an inhibitory rebound mehanism. Second, it appears that it is not the number of MNTB spikes that produces the temporally precise response in the SPON, but rather the timing of the MNTB spikes.

\section{How is the offset response formed?}

Neurons responding at the offset of a stimulus are not a rare finding in the mammalian nervous system. OFF neurons have been described in the retina and lateral geniculate nucleus (Hubel and Wiesel 1961; Schiller and Malpeli 1978) and at several levels of the auditory pathway including the MNTB and DMPO (Guinan et al. 1972), MSO (Grothe 1994), VNLL (Guinan et al. 1972; Batra and Fitzpatrick 1999), DNLL (Bajo et al. 1998), IC (Faingold et al. 1986), medial geniculate nucleus (He 2001) and auditory cortex (He et al. 1997; He 2001). Moreover, inhibition timed to the offset of acoustic stimuli has been reported in DNLL (Bajo et

al. 1989; Bauer et al. 2000) and IC (Faingold et al., 1986) and is at least partially mediated by GABA (Faingold et al. 1986; Bauer et al. 2000). Thus, it appears that offset synaptic activity may play an important role in central processing of sensory information. 
We propose two possible mechanisms to account for the offset responses of SPON units. First, the offset response may arise from a long latency excitatory input to SPON, or one that arrives coincident with the offset. A descending projection to SPON from the tectocommissural column (TCC) of the midbrain has recently been described (Viñuela and Saldana 2001) which could represent a long latency input to the nucleus. However, preliminary observations suggest that this projection is probably inhibitory (our own unpublished observation). Furthermore, when presented with long tone stimuli (one second in duration) SPON neurons maintained offset activity, suggesting that a long latency excitatory input may not be involved in the offset response. Therefore, we favor the second possible mechanism: that the offset response of SPON neurons is caused by a rebound from inhibition during the stimulus. Consistent with this idea are immunocytochemical data showing that SPON neurons receive dense axo-somatic and axodendritic glycinergic and GABAergic inhibition (Kulesza and Berrebi 2000). The precise role of each inhibitory neurotransmitter in the generation of the offset response is currently being investigated through selective pharmacologic blockade of their receptors using a multi-barrel recording electrode configuration.

In the basal ganglia and thalamus, so-called "off" neurons reportedly generate action potentials following a hyperpolarization, and this phenomenon has been termed "post-inhibitory rebound" (Plenz and Kital 1999; Bando et al. 1980; Grenier et al. 1998). Post-inhibitory rebound has been attributed to the opening of low-threshold, voltage-gated $\mathrm{Ca}^{2+}$ channels or to $I_{\mathrm{h}}$, a hyperpolarization-activated cationic current (Aizenman and Linden 1999; Cooper and Standford 2000). Low-threshold, voltage gated $\mathrm{Ca}^{2+}$ channels are reportedly de-inactivated during hyperpolarization, such as that which would be provided by a strong glycinergic inhibition, and open on the return to more depolarized membrane potentials. This results in an influx of calcium and the generation of a $\mathrm{Ca}^{2+}$ spike that may be capped by $\mathrm{Na}^{+}$spikes. The calcium channels may be required to generate the offset-chopper and offset-sustained responses in the SPON, while sodium spikes may be sufficient to account for responses of offset-transient units. Interestingly, in the subthalamic nucleus of the rat, rebound bursts have been elicted in vivo by application of GABA (Plenz and Kital, 1999), indicating that a hyperpolarizing inhibitory input alone is sufficient to generate this response. 


\section{Effects of GABA on response properties of inferior colliculus neurons}

The multitude of synaptic inputs arriving at the central nucleus of the IC are both excitatory and inhibitory, and it is well documented that GABAergic inhibition has a profound impact on response properties of IC neurons. For example, GABAergic inputs are required for interaural intensity disparity coding, shape PSTH response properties and tuning curves (Yang et al. 1992; Koch and Grothe 1998) and lengthen first-spike latencies of IC neurons (Park and Pollak 1993; LeBeau et al. 1996). GABA has also been shown to sharpen response maps and is essential for duration tuning in the IC (Casseday et al. 1994; Fuzessery and Hall 1996).

Our data also correspond with previous reports of a contralaterally evoked offset inhibition to the rat IC that appears to be mediated by GABA (Faingold et al. 1986). When the $\mathrm{GABA}_{A}$ receptor antagonist bicuculline was applied to IC units, their responses were transformed from "ON" neurons into "ON/OFF" neurons, suggesting that GABA normally suppresses their offset activity. Interestingly, "ON/OFF" neurons are more common in the IC of the genetically epilepsy-prone rat, indicating compromised GABAergic inhibition in these animals. Faingold and colleagues (1986) deduce that the IC is a potential site of seizure foci in genetically epilepsy-prone rats. This suggests that SPON-derived GABAergic inhibition, timed to the stimulus offset, may contribute to suppression of seizure activity in the IC.

Many naturally occurring rat vocalizations, including sounds elicited in threatening situations or in the context of sexual behaviors, consist of a series of 2 to 20 constant frequency calls in the $18-32 \mathrm{kHz}$ range of varying duration (ranging from $20 \mathrm{msec}$ to nearly 4 seconds; Brudzynski et al. 1993). The combination of response features that we have described for the SPON in this report suggests that this cell group is particularly well suited for a role in signaling the end (offset-transient) or duration (offset-choppers and offset-sustained) of species-specific vocalizations.

It also stands to reason from our data that SPON neurons may also serve to detect gaps between closely placed sounds or components of sounds. Detection of gaps or silent periods 
between stimuli plays an important role in processing complex sounds and in speech perception (Barsz et al. 1998) and may be encoded by suppressing activity during gaps through inhibition. Neurons in the mouse IC can detect gaps between two stimuli that are as short as 2 ms (Walton et al. 1997). Many IC neurons show post-stimulus suppression that is mediated by GABA and lasting up to $270 \mathrm{~ms}$ after the stimulus (Finlayson 1999; Bauer et al. 2000). These findings indicate that existence of a GABAergic input to the IC that arrives at the end of a stimulus or outlasts it by tens of milliseconds. Two likely sources for gap detectors are the neurons of the SPON and GABAergic offset neurons in the IC with intrinsic collateral projections.

The main sources of ascending inhibition reaching the IC are the SPON and the ventral and dorsal nuclei of the lateral lemniscus (VNLL and DNLL). Unbiased stereological estimates indicate that the rat SPON contains approximately 2,400 neurons, the overwhelming majority of which are GABAergic (Kulesza and Berrebi 2000; Kulesza et al. in press). By comparison, the rat's DNLL contains approximately 1,800, presumably GABAergic neurons (Kulesza et al. in press; Mugnaini and Oertel 1985), and the VNLL contains nearly 14,000 neurons, approximately two-thirds of which are inhibitory (Kulesza et al. in press; Riquelme et al. 2001). Thus, the SPON represents a significant source of GABAergic inhibition to the IC. Until now, the potential for modulation of collicular physiology by the SPON has been largely overlooked. 


\section{Acknowledgements}

This work was supported by grants NIDCD DC02266 (ASB) and NSF 97-23963 (GAS). RJK was supported by a teaching assistantship from the West Virginia University School of Medicine, Department of Neurobiology and Anatomy. The authors wish to thank Brian Pope and Jesse Thompson for their technical assistance. We are also grateful for the pre-submission critiques of the manuscript by Drs. Aric Agmon, Peter Mathers and Janet Cyr. 

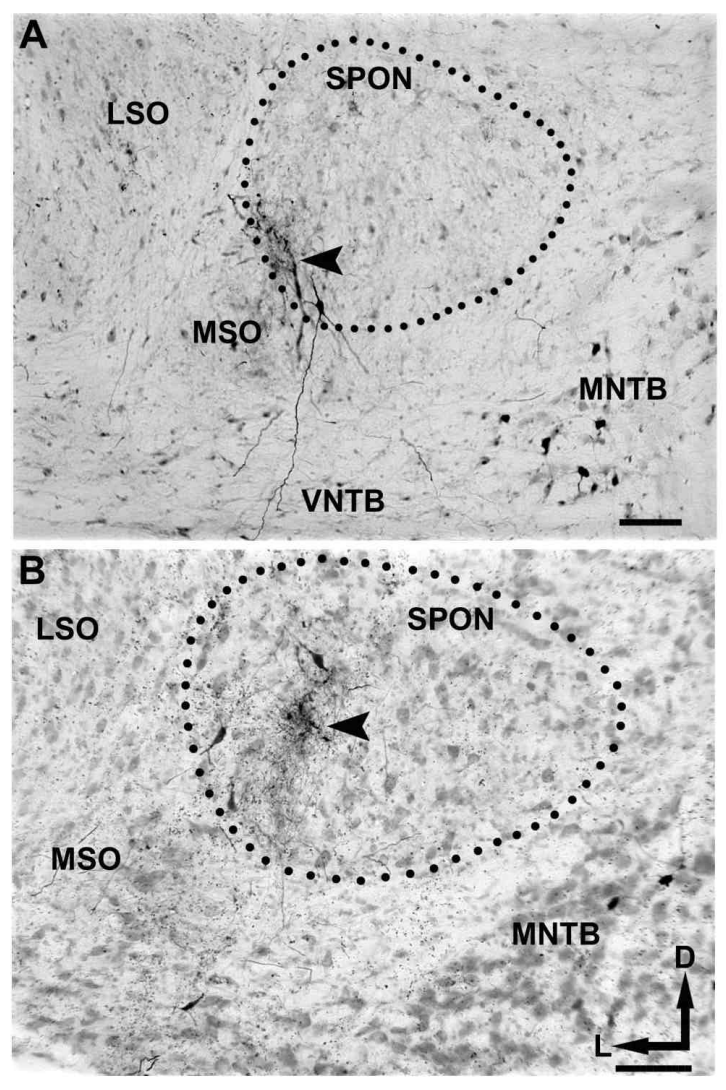

Figure 4.1. Localization of recording sites. Recording sites were marked with small deposits of biocytin. Coronal sections through the superior olivary complex demonstrate two such deposits (arrowheads). In both cases neurons were retrogradely labeled within the SPON and MNTB from the injection site. D, dorsal; L, lateral; LSO, lateral superior olive; MNTB, medial nucleus of the trapezoid body; MSO, medial superior olive; SPON, superior paraolivary nucleus; VNTB, ventral nucleus of the trapezoid body. Scale bar in A and B $=100 \mu \mathrm{m}$. 

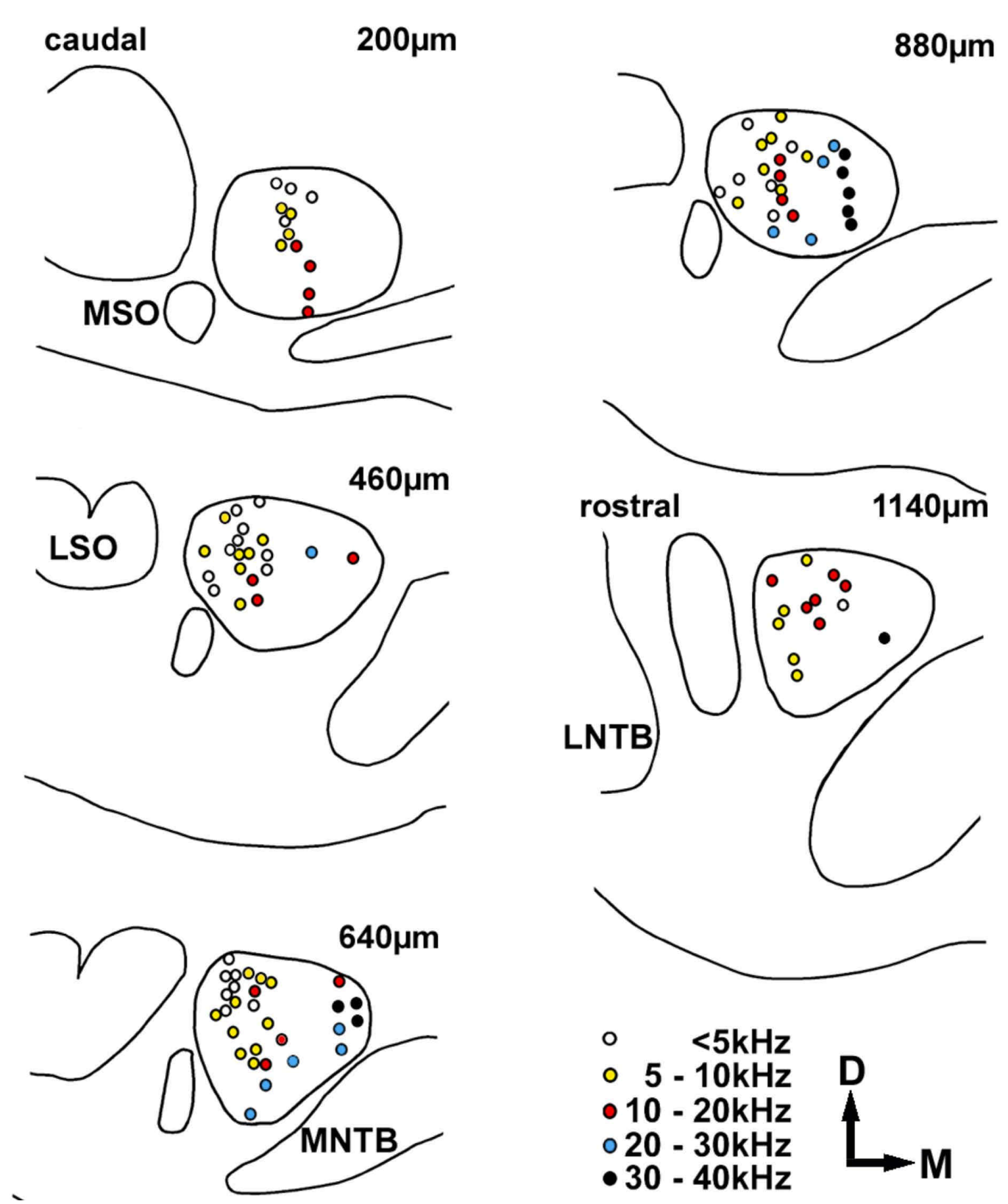

Figure 4.2. Tonotopic arrangement of best frequencies in SPON. Single unit recording sites are plotted by BF on a series of standardized templates spanning the caudal-most (top left) to rostral-most (bottom right) extent of the SOC. The rostro-caudal spacing (number in upper right of each figure) is indicated relative to the caudal end of the SOC. BFs ranged from $1.2 \mathrm{kHz}$ to 40 $\mathrm{kHz}$, and covered the rat's audible range of hearing. Neurons with low BFs $(5-10 \mathrm{kHz})$ are located laterally, those with high BFs $(20-40 \mathrm{kHz})$ are located medially and neurons with intermediate BFs (11-20 kHz) are interposed centrally within the nucleus. LNTB, lateral nucleus of the trapezoid body; M, medial. Other abbreviations as in Figure 1. 
A
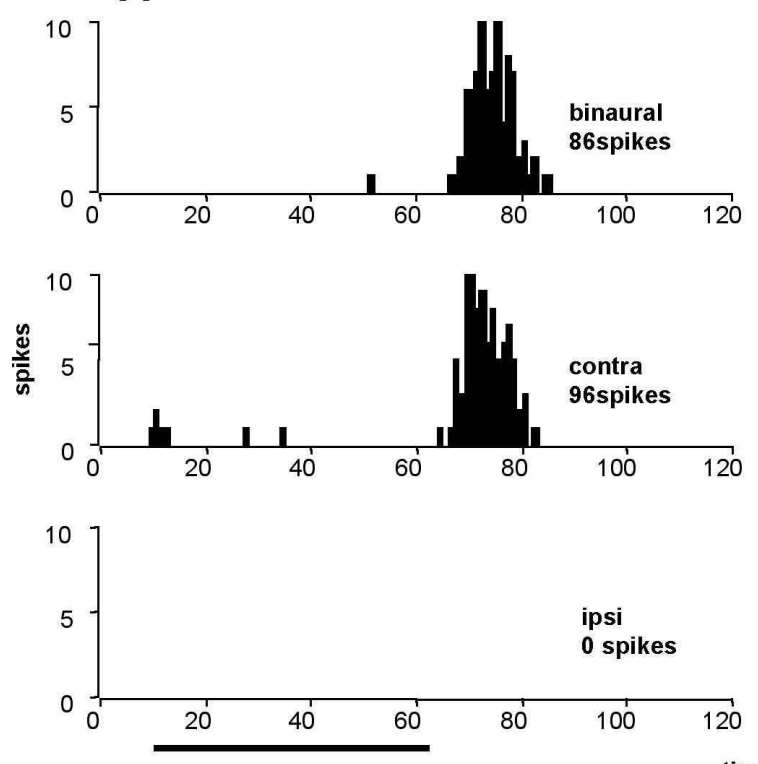

B
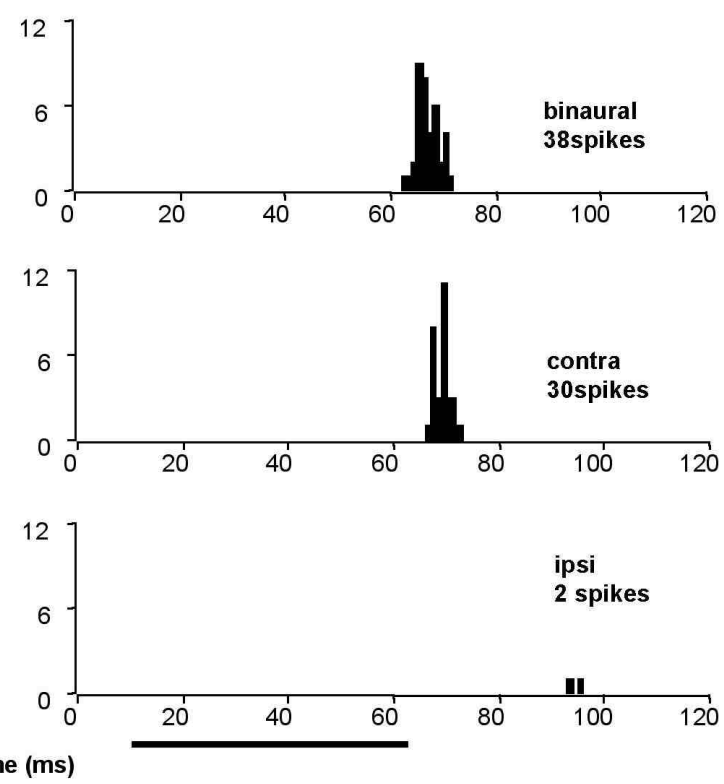

Figure 4.3. Single unit responses to broad band noise. Responses to 20 presentations of a 50 msec long broad band noise stimulus ( $20 \mathrm{~dB}$ attenuation) are shown for two SPON neurons. The overwhelming majority of SPON units did not respond to ipsilateral stimulation, but responded only to contralateral inputs (left, OE). Approximately 13\% of neurons showed binaural facilitation $(0 \mathrm{E}-\mathrm{F})$, where the binaural response contained significantly more spikes than the contralateral response (right). 

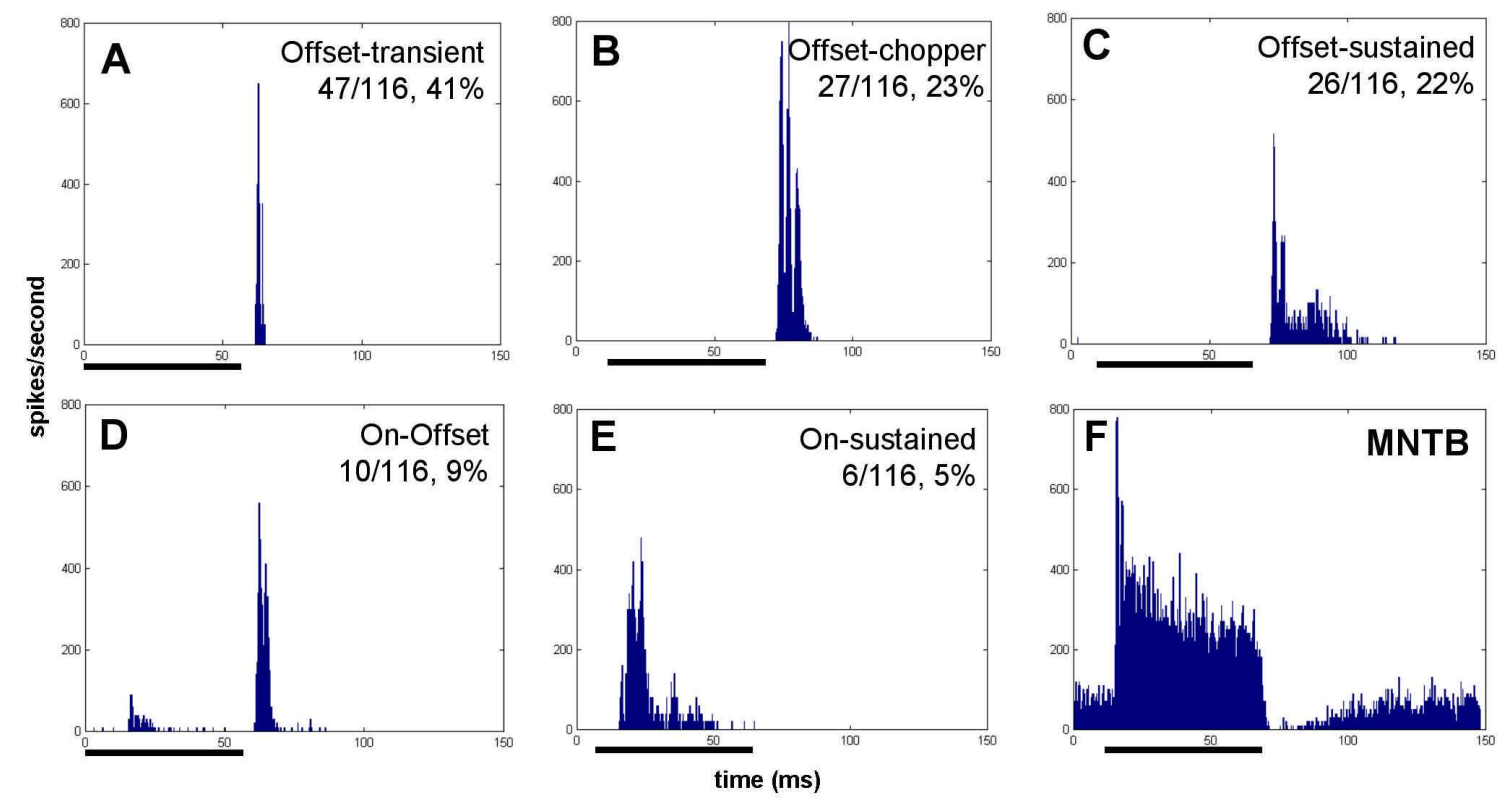

Figure 4.4. SPON response types. The vast majority of SPON neurons (100 of 116, 86\%) respond to $\mathrm{BF}$ tones only with offset responses. These offset responses could be divided into groups consisting of a single spike (A, $41 \%$ of units), those consisting of two or more equally separated spikes (B, 23\% of units) and those with activity lasting more than 20 milliseconds (C, $22 \%$ of units). A total of 10 cells responded with an offset response that was preceded by a few spikes during the stimulus presentation (D, 9\% of units). The remaining 6 cells displayed onset or sustained responses during the tone (E, 5\% of units). A PSTH from an MNTB neuron (F) displaying typical primary-like response is provided to show their high rate of spontaneous activity, increase in activity during stimulation and quiescent period following stimulation. 


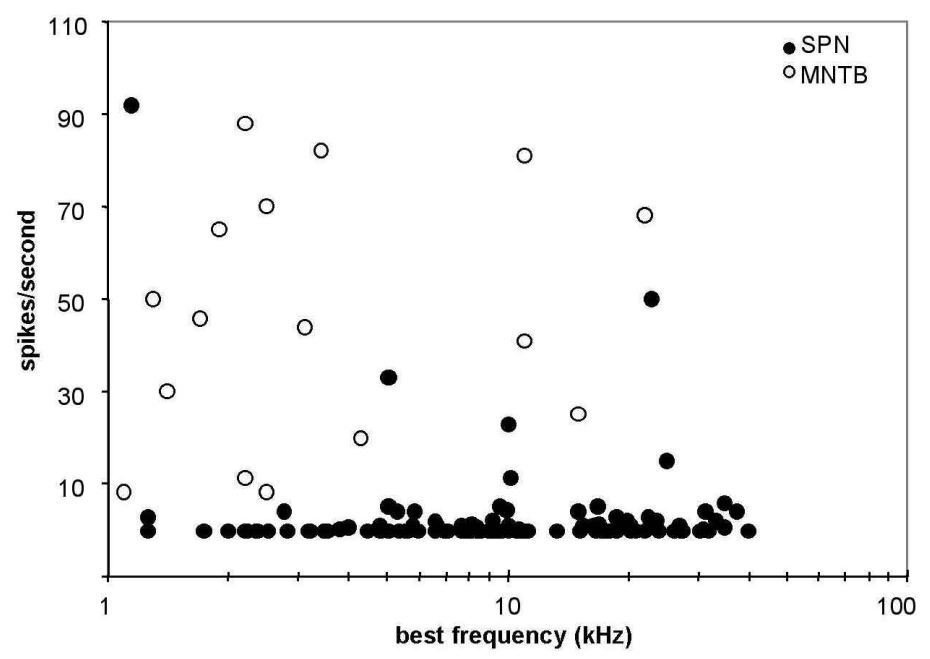

Figure 4.5. SPON units have very low rates of spontaneous activity. The majority of SPON units (closed circles) had spontaneous rates below five spikes per second. Data from MNTB units (open circles) are included for comparison. 


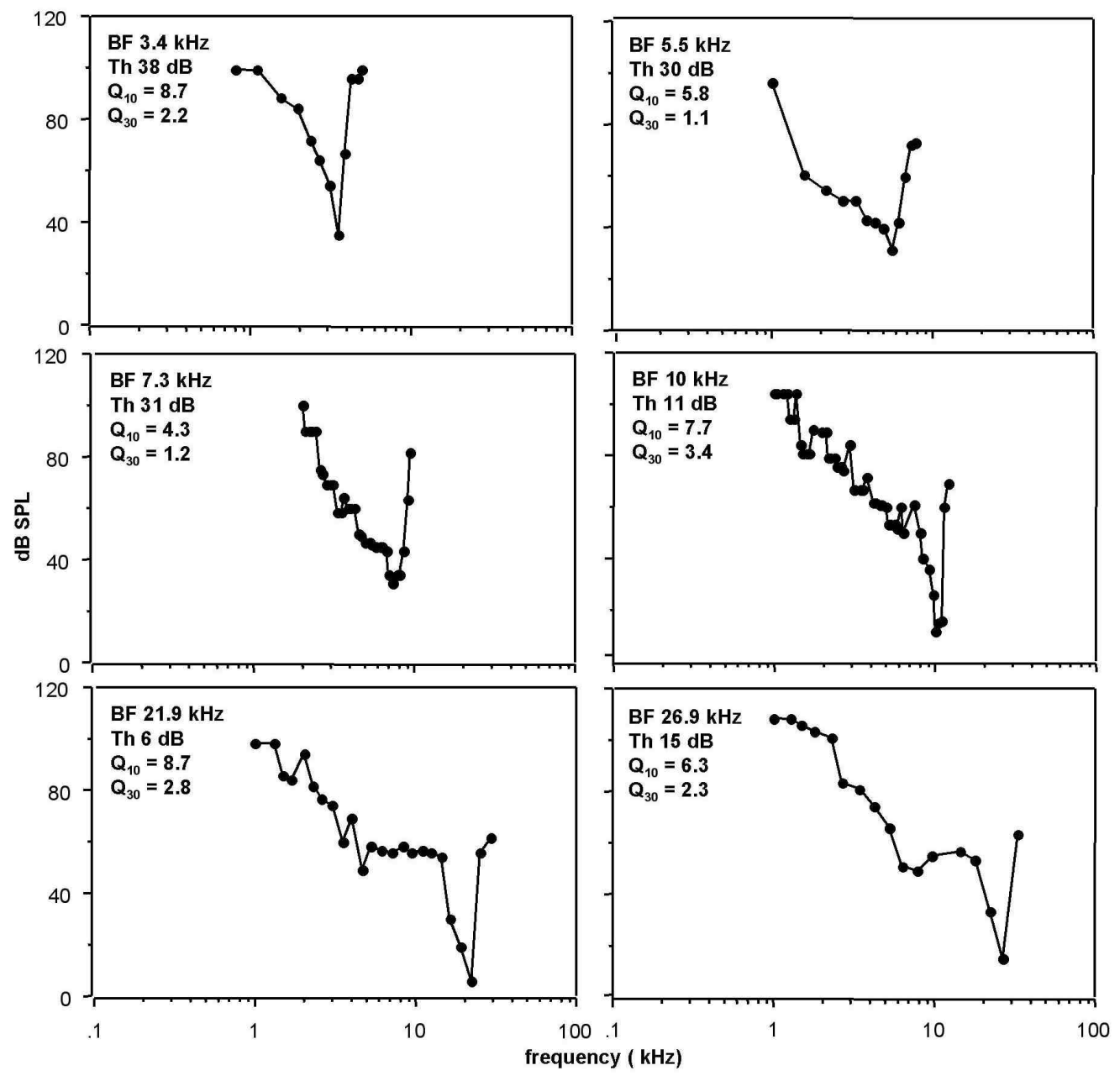

Figure 4.6. Response maps. Response maps of six SPON units, ranging in BF from $3.4 \mathrm{kHz}$ to $31 \mathrm{kHz}$. In most cases, the tuning curves display narrow, V-shaped peaks near BF and low frequency tails. For these six units, thresholds $(\mathrm{Th})$ ranged from $22 \mathrm{db}$ to $45 \mathrm{~dB}$. Sharpness of tuning for each unit was also determined by calculating $\mathrm{Q}_{10}$ and $\mathrm{Q}_{30}$ values. 
1
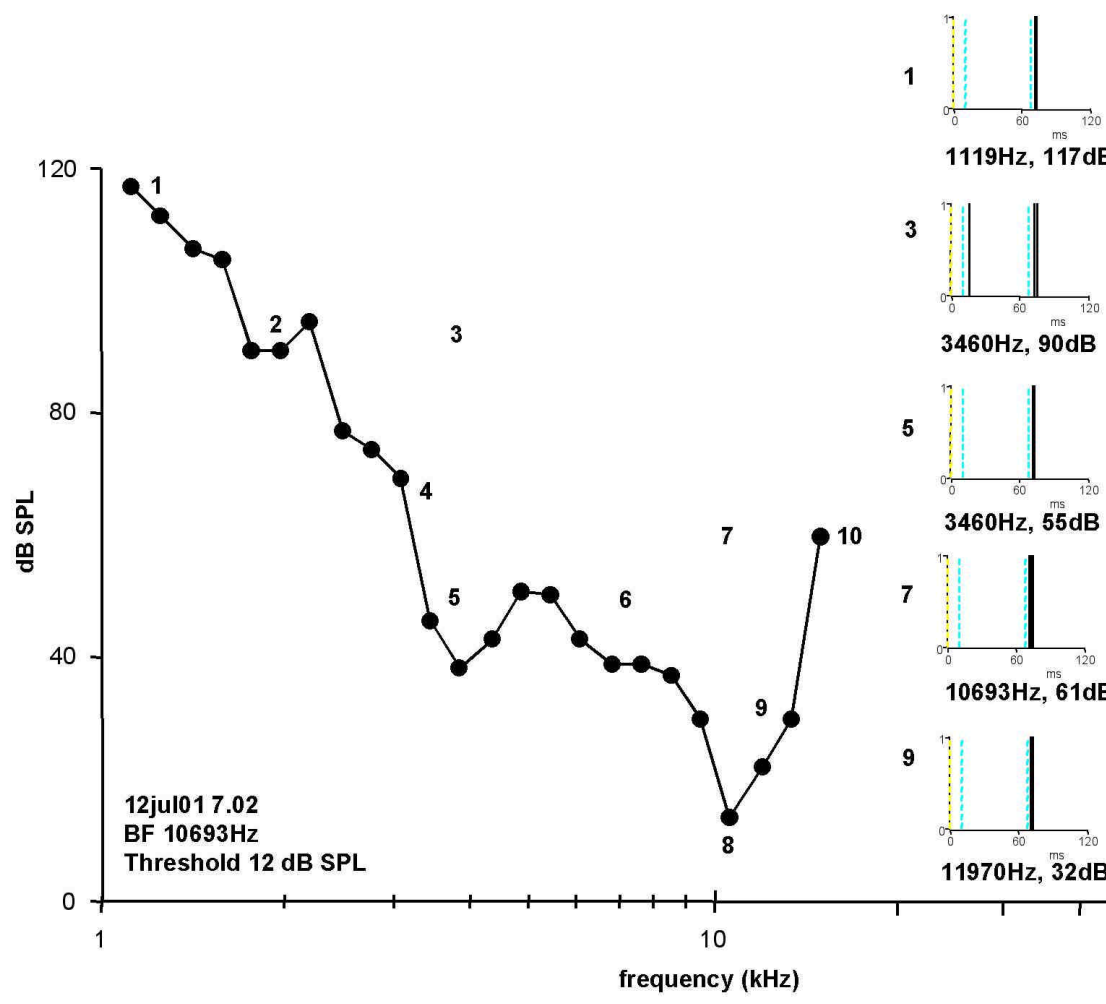

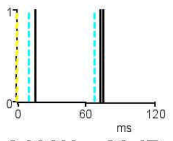

$3460 \mathrm{~Hz}, 90 \mathrm{~dB}$$$
\text { . }
$$

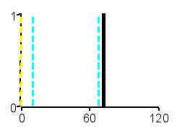

$3460 \mathrm{~Hz}, 55 \mathrm{~dB}$

7

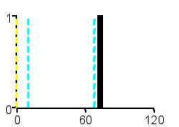

$10693 \mathrm{~Hz}, 61 \mathrm{~dB}$

9

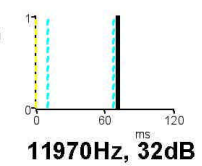

2

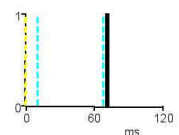

$1976 \mathrm{~Hz}, 89 \mathrm{~dB}$

4

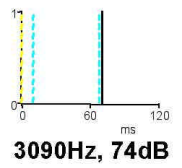

6

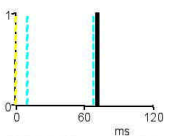

$6809 \mathrm{~Hz}, 54 \mathrm{~dB}$

8

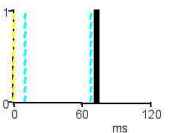

$10693 \mathrm{~Hz}, 14 \mathrm{~dB}$

10

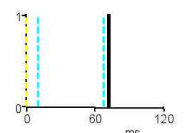

$15000 \mathrm{~Hz}, 60 \mathrm{~dB}$

Figure 4.7. Peristimulus time histograms demonstrate that offset responses occur throughout the response maps of the units. The left part of the figure depicts the response map of a unit whose BF was $10.7 \mathrm{kHz}$, and whose threshold was $31 \mathrm{~dB}$ SPL. At 10 locations within the unit's response map, we constructed a PSTH from its response to a single repetition of a $50 \mathrm{~ms}$ tone (shown at right, stimulus parameters are given under each PSTH, vertical bars indicate the stimulus duration). Note that a consistent offset response is found; only at very high intensity levels (90 dB; PSTH \#3) did we observe onset spikes. 


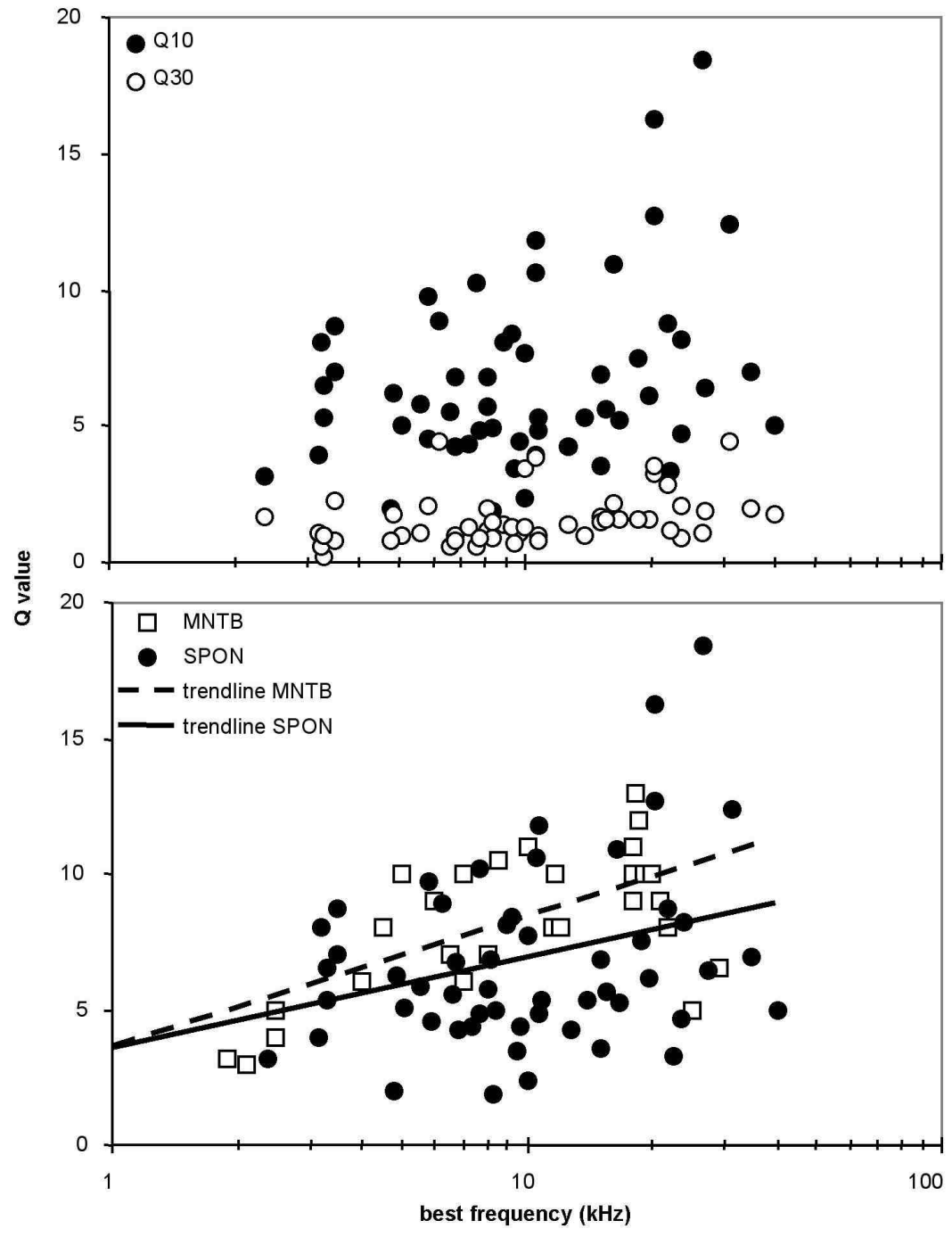

Figure 4.8. SPON neurons have narrow tuning curves. In the top panel, $\mathrm{Q}_{10}$ (closed circles) and $\mathrm{Q}_{30}$ (open circles) values of tuning curve sharpness for the population of units are plotted according to $\mathrm{BF}$. Average $\mathrm{Q}_{10}$ values were $6.77 \pm 3.30$ and average $\mathrm{Q}_{30}$ values were $1.64 \pm 0.99$. In the bottom panel, $\mathrm{Q}_{10} \mathrm{~S}$ are compared between SPON and MNTB (Guinan, 1968). The trend lines indicate that SPON neurons have only slightly wider receptive fields than MNTB neurons. 


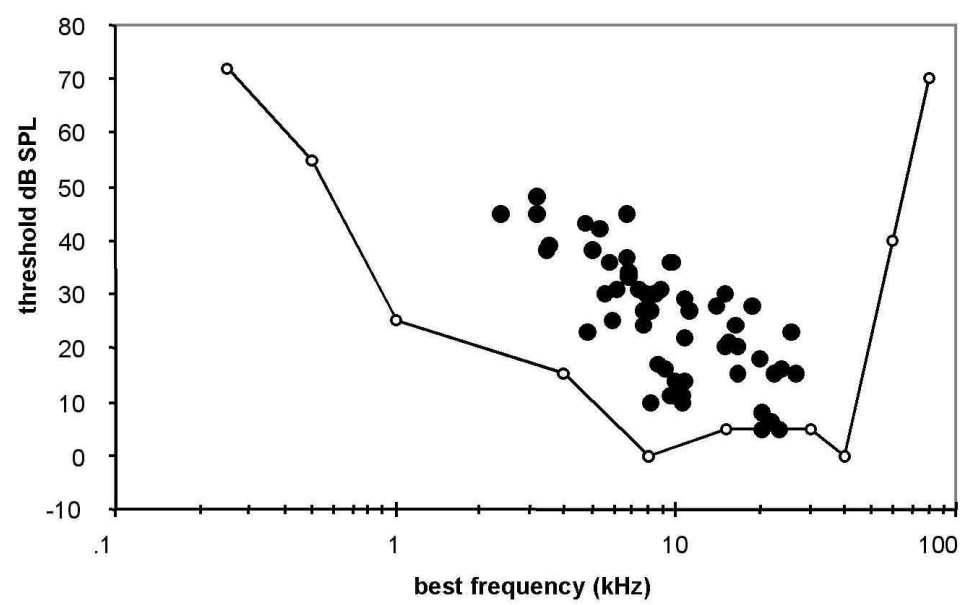

Figure 4.9. Thresholds of SPON units. The thresholds of 60 SPON units at BF averaged $25 \pm$ 11.43 dB SPL and ranged from 5-48 dB SPL. A behavioral audiogram for the albino rat, determined from the literature (Kelly and Masterton, 1977), is included for comparison. 

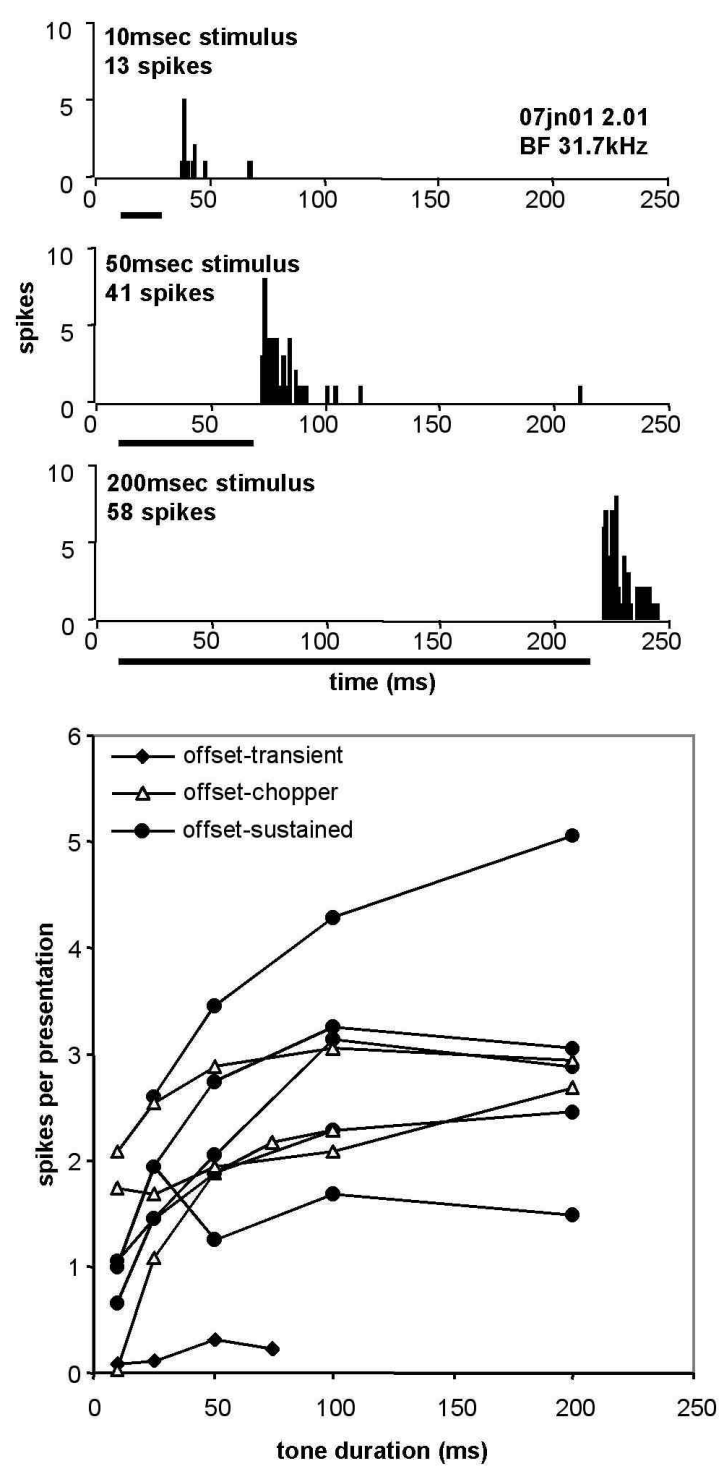

Figure 4.10. Duration sensitivity. Nearly half of all SPON neurons showed duration sensitivity. The PSTHs (top) are responses to 10, 50 and $200 \mathrm{msec}$ from one neuron. This particular cell responded with 0.65 spikes per sweep for the $10 \mathrm{msec}$ tone, but fired 3.15 spikes per stimulus during the $100 \mathrm{msec}$ tone. Duration tuning curves (bottom) are shown for the nine neurons that showed long duration sensitivity to pure tone stimuli, $20 \mathrm{~dB}$ above threshold and plots the average number of discharges over twenty repetitions against tone duration. This small sample contains units from all offset response classes (mostly offset-choppers and offsetsustained units). 


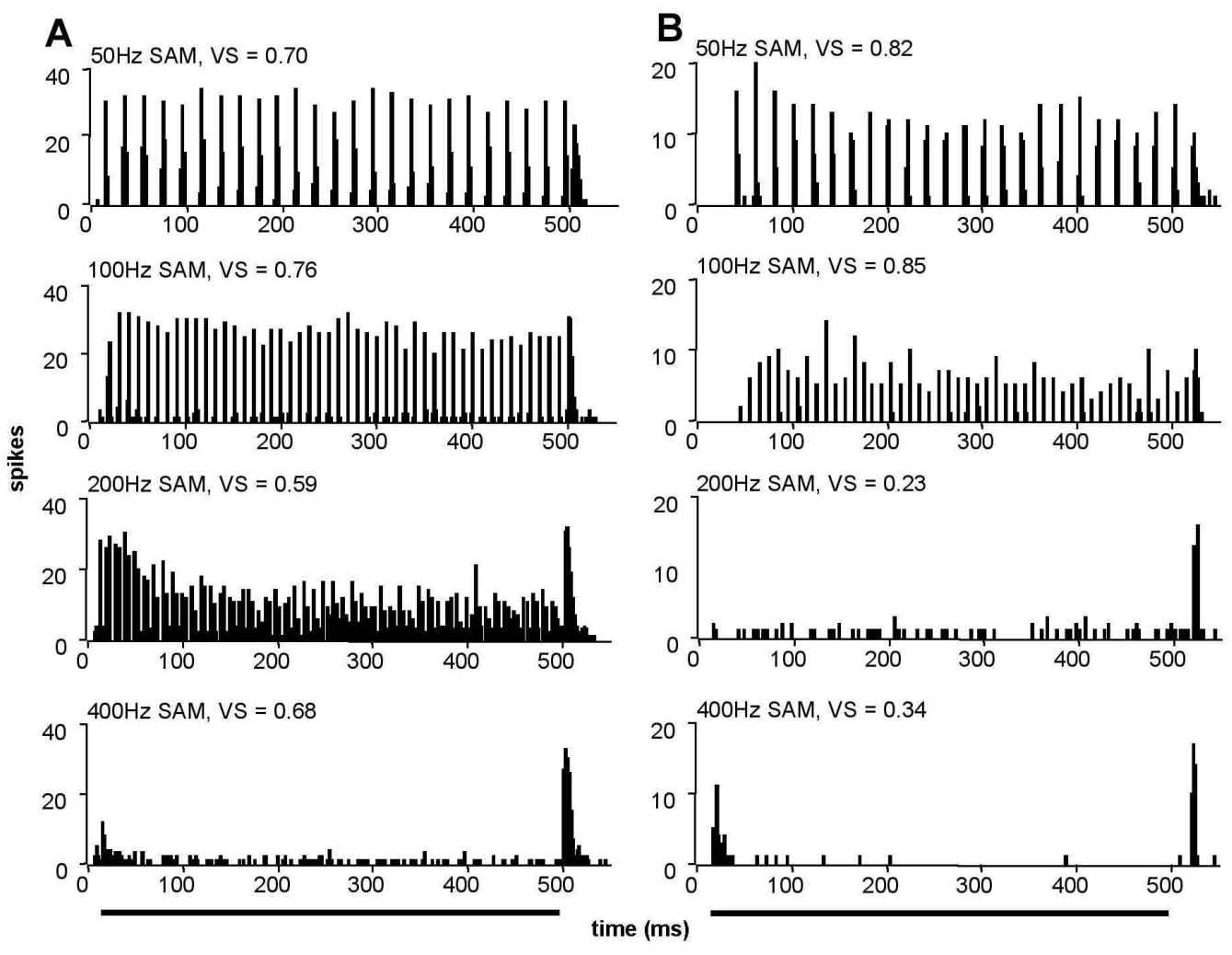

Figure 4.11. SPON unit responses to amplitude modulated tones. PSTHs for two units are shown in response to $500 \mathrm{~ms}$ amplitude modulated tones at 50,100, 200 and $400 \mathrm{~Hz}$ rates of modulation. Vector strengths (VS) are included with each PSTH. At each modulation rate, note the prominent offset response. Both units (A and B) demonstrate high-fidelity phase-locking to the stimulus envelope up to $100 \mathrm{~Hz}$ modulation, but vector strengths decline at higher modulationrates $(200 \mathrm{~Hz}$ for the unit depicted in B). However, even at $400 \mathrm{~Hz}$ modulation many units had relatively high vector strengths (A) because they fired well-timed, albeit fewer, spikes to the SAM stimulus. 


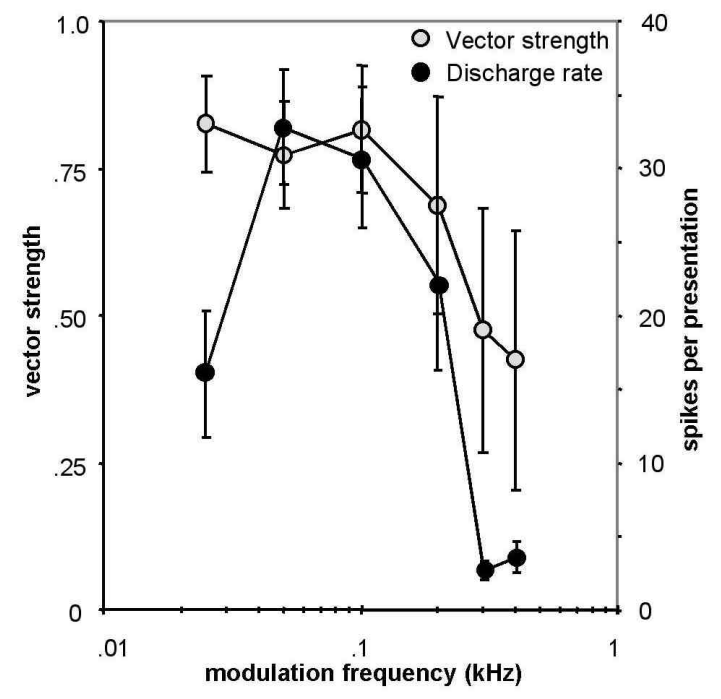

Figure 4.12. Plots of discharge rates and vector strengths for SAM stimuli for the population of SPON units. Discharge rates (dark circles) increase from 25 to $50 \mathrm{~Hz}$, then decline to near zero spikes at 300 and $400 \mathrm{~Hz}$ modulation. Vector strengths (grey circles) are relatively high (>0.75) for low frequency modulations $(25-100 \mathrm{~Hz})$ but begin to fall at higher frequencies $(200 \mathrm{~Hz})$. 


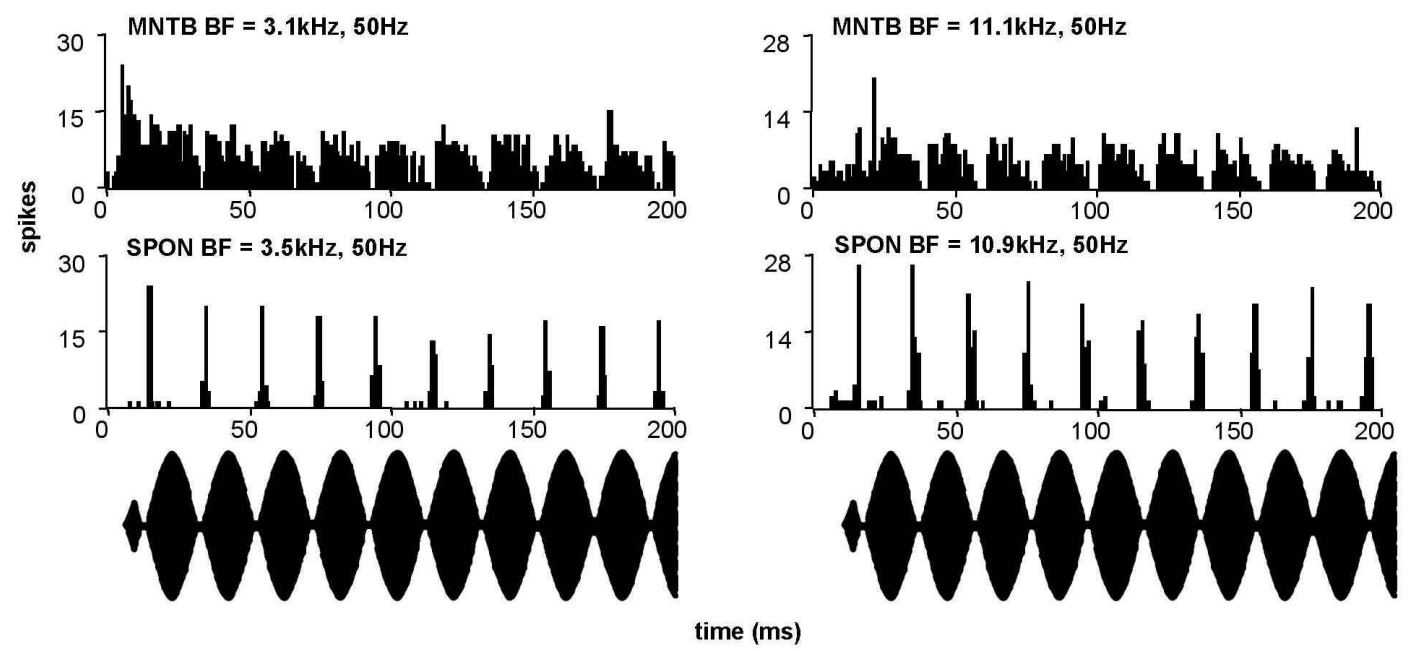

Figure 4.13. Comparison of MNTB and SPON responses to SAM Responses to 20 presentations of a $50 \mathrm{~Hz}$ modulated BF tone from similarly tuned pairs of MNTB and SPON units are shown. Below each pair, the stimulus is represented schematically. The MNTB unit responds during the entire length of the modulation cycle and is quiet only between cycles. The SPON unit only responds in between cycles, when the MNTB is inactive. 


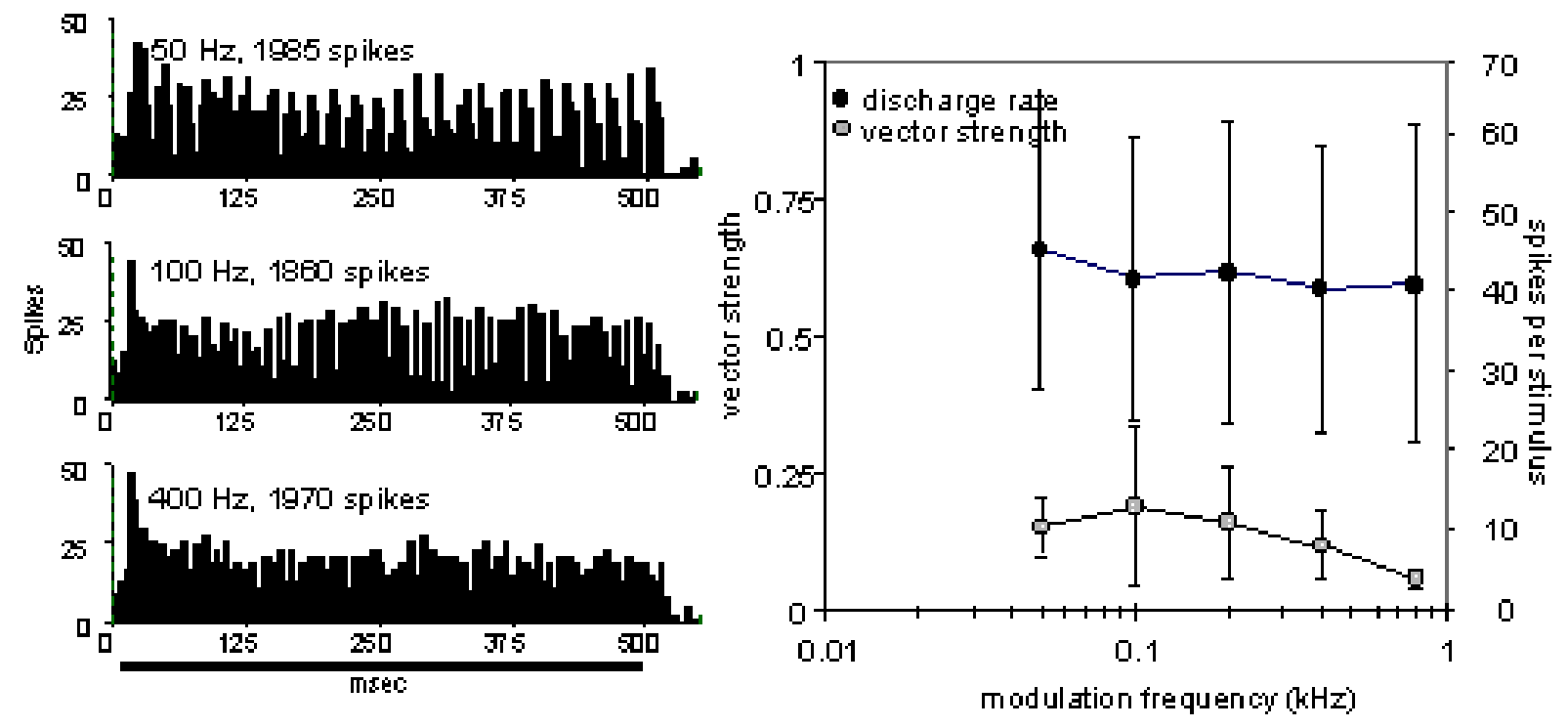

Figure 4.14 Responses of MNTB neurons to SAM MNTB neurons were sensitive to low frequency amplitude modulation and followed the envelope of SAM stimuli, up to 100 Hz. At $200 \mathrm{~Hz}$ modulation frequency and higher, MNTB neurons responded in a sustained manner that did not reflect the stimulus envelope. The number of discharges to amplitude modulated stimuli was relatively constant across modulation frequencies and the sustained responses yielded relatively low vector strengths. 
Chapter Five

The effects of GABA and glycine on response properties of superior paraolivary nucleus neurons in rat 


\begin{abstract}
The rat superior paraolivary nucleus (SPON) is a group of neurons situated in the superior olivary complex, a constellation of nuclei involved in auditory processing. The SPON contains multipolar, GABAergic neurons that receive both GABAergic and glycinergic inhibitory innervation. Most SPON neurons respond only to stimulation of the contralateral ear with spike activity timed to the stimulus offset. However, it remains unclear how the offset response is formed and the effects of GABAergic and glycinergic inhibition on response properties of SPON neurons are unknown.
\end{abstract}

To examine the role of inhibitory inputs on SPON response properties, we made extracellular recordings in vivo with multibarrel electrodes before, during and after local application of bicuculline (a GABA $\mathrm{G}_{\mathrm{A}}$ receptor antagonist), strychnine (a glycine receptor antagonist) and a cocktail of the two inhibitory antagonists. Blockade of the $\mathrm{GABA}_{\mathrm{A}}$ receptor resulted in an increase in the number of spikes occurring at the offset of pure tone stimuli; a few neurons responded weakly during the stimulus. Blocking the glycine receptor with strychnine abolished the offset response to pure tones and transient responses timed to the stimulus onset were observed. When the $\mathrm{GABA}_{\mathrm{A}}$ and glycine receptors were blocked simultaneously, offset responses were abolished and neurons responded throughout the duration of the stimulus. Additionally, blockade of the $\mathrm{GABA}_{\mathrm{A}}$ and glycine receptors resulted in broader receptive fields and responses to amplitude modulation were poorly timed. These data provide evidence that inhibition plays a prominent and essential role in the formation of SPON responses to sound and that the offset response arises from a postinhibitory rebound mechanism. 


\section{INTRODUCTION}

In our immunocytochemical investigation for the presence of the inhibitory neurotransmitters GABA and glycine (chapter 2) we showed that SPON neurons receive a strong inhibitory innervation. In the previous chapter (chapter 4) we demonstrated that SPON neurons respond at the offset of pure tone and broad band noise stimuli. However, spontaneous rates of activity were very low, making the effects of inhibitory input difficult to study. Thus, the effects of the massive convergence of inhibitory inputs on the physiological responses of SPON neurons remain unknown. Moreover, the mechanism that leads to offset responses in the SPON is unclear. It is well established that the rat SPON receives its excitatory input from the octopus and mulitipolar cells of the contralateral cochlear nucleus (Friauf and Ostwald, 1988; Kuwabara and Zook, 1991; Thompson and Thompson, 1991; Banks and Smith, 1992; Schofield and Cant, 1995). Octopus cells are described as responding only at the stimulus onset with fast, precisely time spikes (Godfrey et al., 1975, Rhode et al., 1983); multipolar neurons are typically described as having chopper responses, where the neurons fires a series of spikes with relatively constant interspike intervals (Smith and Rhode, 1989). The SPON also receives a strong, sustained glycinergic input from the MNTB (Banks and Smith, 1992; Sommer et al., 1993; Schofield and Cant, 1995) and a GABAergic input that arises partially from collaterals of SPON axons, the tecto-commissural column and from other unidentified sources. Thus, it is unclear if the offset response simply reflects an undescribed input or results from integration of excitatory and inhibitory inputs. However, we have arrived at two possible mechanisms that might be involved in creating offset responses. 1. The offset response may be generated by an excitatory input that arrives with a long latency relative to the stimulus onset or arrives coincident with the stimulus offset. SPON neurons receive a descending input from the tectocommissural column (TCC), a portion of which may be excitatory (Viñeula and Saldaña, 2001) and contribute a delayed or long latency excitatory influence. 2. We propose that the offset responses recorded from SPON neurons result from inhibitory rebound, where the removal of a hyperpolarizing, inhibitory input (at the end of stimulus) can trigger rebound spikes. In rat, SPON neurons receive massive glycinergic and GABAergic inhibitory inputs (Kulesza and Berrebi, 2000). Measurements of amino acid concentrations in the SPON indicate that this region contains the highest levels of GABA and glycine in the SOC (Godfrey et al., 2000). Glycinergic input arises from the 
ipsilateral medial nucleus of the trapezoid body (MNTB), suggesting this inhibition has high rates of spontaneous activity (providing inhibitory influence in the absence of sound), is temporally precise and phase locked to amplitude modulations.

In this chapter, we examine the role of inhibition in SPON responses to pure tones, by recording from neurons with multibarrel micropipettes, in the presence of bicuculline (a GABA receptor antagonist; Curtis et al., 1971b) and strychnine (a glycine receptor antagonist; Curtis et al., 1971a) and comparing those results to control data from the same neuron. In addition, we examined the effect of inhibitory neurotransmission on receptive fields (response maps) and responses to sinusoidally amplitude modulated pure tones (SAM). By examining the spike rate of SPON cells before, during and after stimulus presentation we can precisely dissect the effects of different types of inhibition. Our data provide evidence that GABAergic inhibition arrives coincident with stimulus offset, observed as an increase in the number of spikes at the offset without discharges during the stimulus. However, offset activity was abolished in the presence of strychnine suggesting that offset spikes are a rebound from glycinergic inhibition. Additionally, responses recorded in the presence of both antagonists suggest that inhibition sharpens response maps and that excitatory input to the SPON is sustained with long first spike latencies relative to the stimulus onset. Responses to SAM pure tones provide evidence that the fine temporal sensitivity to the stimulus envelope observed in control conditions is at least in part a result of inhibitory influence. 


\section{METHODS}

\section{Stereotaxic surgery}

Twenty-three female, Sprague-Dawley albino rats, weighing between 230 and 300 grams were used for this study. Animals were anesthetized with a mixture of xylazine and ketamine (8.6 mg and $57 \mathrm{mg}$ per $\mathrm{kg}$ body weight, respectively). Once animals were areflexic, a midline incision was made through the scalp and the pinnae were reflected bilaterally. Animals were restrained in a stereotaxic instrument and custom-built hollow brass earbars, serving as sound delivery tubes, were inserted into the cartilaginous external auditory meatus. A hole was drilled through the postparietal bone, the dura mater was incised and the cerebellar tissue overlying the floor of the fourth ventricle was removed by aspiration. The anesthetic state of the animal was monitored throughout the experiment and supplemental doses of the same anesthetics were given at 2/5 the original dose, as needed.

\section{Stimuli and recording}

All recording procedures were performed inside a sound attenuated booth. Stimuli were created digitally using Tucker-Davis Technologies System III Virtual Design Studio (TuckerDavis Technologies, Gainsville, FL). Digital stimuli were converted to analog signals with a System 3 Real Time Processor (RP2, Tucker-Davis Technologies, Gainsville, FL), sent to a

programmable switch attenuator and then through a weighted summer. Intensity (in $\mathrm{dB}$ attenuation) of the right and left signals were controlled through separate attenuators. The sound signal was then relayed to amplifiers and presented via custom-built Stax speakers that fit onto the ends of the brass earbars (Sokolich, 1981). To permit calibration of the sound delivery system, the hollow ear bars were machined with a small calibration tube that joined the sound delivery tube at a $45^{\circ}$ angle. Prior to or following an experiment, a B \& $\mathrm{K}$ microphone was placed into the calibration tube and the sound delivery system calibrated for pure tones between 1 and $40 \mathrm{kHz}$. Stimulus intensities were converted to $\mathrm{dB}$ SPL offline and corrected for augmentation or attenuation caused by the B \& K microphone.

Electrodes were advanced from outside the sound attenuated booth with a Burleigh 
Inchworm microdrive (Burleigh Instruments, Inc., Victor, NY). Action potentials from single units were fed through an amplifier to a spike conditioner, converted to a digital signal through an A/D converter and displayed using Brainware software (RP2; Tucker-Davis Technologies, Gainsville, FL). All recordings were made with "piggyback" electrodes (Havey and Caspary, 1980). Recording electrodes were pulled from single barrel glass micropipettes and tips were broken back to an outside diameter of $2.5 \mu \mathrm{m}$ (10-20M). A five-barrel pipette (World Precision

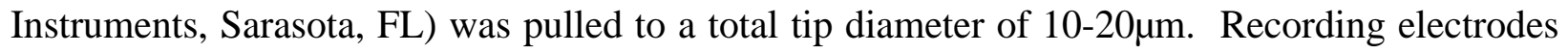
were mounted onto the five-barrel pipettes so the tip of the recording electrode extended approximately $10 \mu \mathrm{m}$ past the tip of the five barrel pipette. Recording pipettes were filled with a solution of $3 \mathrm{M} \mathrm{KCl}$ with $2.5 \%$ biocytin. We used bicuculline, a selective antagonist at the $\mathrm{GABA}_{\mathrm{A}}$ receptor to block GABAergic input (10mM, Sigma). To block glycinergic input, we used strychnine, a competitive antagonist at the glycine receptor (10mM, Sigma). To block both the $\mathrm{GABA}_{\mathrm{A}}$ and glycine receptors simultaneously, we used a cocktail of bicuculline and strychnine (both $10 \mathrm{mM}$ ). Each drug was dissolved in $0.165 \mathrm{M} \mathrm{NaCl}, \mathrm{pH} 3$ and retained in one barrel of the five barrel pipette with a $-15 \mathrm{nA}$ current. Bicuculline was delivered with positive currents that ranged from 15 to $25 \mathrm{nA}$, strychnine was delivered with positive currents that ranged from 20 to $36 \mathrm{nA}$ and the cocktail of bicuculline and strychnine was delivered with positive currents ranging from 30 to $40 \mathrm{nA}$. A second barrel of the five barrel pipette was used as a ground channel and was filled with $0.9 \% \mathrm{NaCl}$. We observed no effect on neuronal discharge during iontophoresis of saline or drug vehicle $(0.165 \mathrm{M} \mathrm{NaCl}, \mathrm{pH} 3)$.

Broad band noise bursts (containing frequencies from $20 \mathrm{~Hz}$ to $61 \mathrm{kHz}, 50 \mathrm{~ms}$ duration) were used to search for single units. Response maps were compiled from single responses to numerous frequency-intensity combinations of pure tone stimuli. Best frequency (BF), defined as the frequency to which the neuron responded at the lowest stimulus intensity, was derived from the response map for each unit. Threshold was determined visually while stepping through a $\mathrm{BF}$ tone in $1 \mathrm{~dB}$ increments, and was defined as the lowest intensity at which the neuron responded. $\mathrm{Q}_{20}$ values were calculated by dividing a neurons $\mathrm{BF}$ by the bandwidth of the response map (for that neuron) at $20 \mathrm{~dB}$ above the neurons threshold. Peristimulus time histograms (PSTHs) to BF tones, $20 \mathrm{~dB}$ above threshold (5ms cosine $\mathrm{e}^{2}$ ramp; $50 \mathrm{~ms}$ duration) were compiled from data collected over 100 repetitions. Spontaneous activity was measured in a 
ten ms window prior to pure tone presentations, for a total time of one second. Sinusoidally amplitude modulated BF tones (100\% modulation), 0.5 seconds in duration, were presented 20 times at each modulation frequency $(50,100,200,400$ and $800 \mathrm{~Hz})$. Synchronization of the response to the modulation frequency was quantified by calculating vector strength (Goldberg and Brown, 1969) at each modulation frequency using the following equation

$$
V S=\sqrt{\left[\sum \sin \left(a_{i}\right)\right]^{2}+\left[\sum \cos \left(a_{i}\right)\right]^{2}} / N
$$

where $a_{i}$ is the phase of spike $i$ relative to the frequency modulation and $\mathrm{N}$ is the total number of spikes. A vector strength of 1 indicates perfect entrainment between the neurons response and the modulation phase, while a vector strength of zero means there is no correlation between the neurons response and the modulation phase. Offset spikes were excluded from the calculation of vector strength.

After baseline data were collected (response map, BF, threshold, pure tone and SAM PSTHs) drugs were delivered to the neuron. For each neuron, the number of discharges in response to 100 repetitions of a $\mathrm{BF}$ tone, $20 \mathrm{~dB}$ above threshold, was monitored every two to five minutes until the discharge rate reached a plateau (between 10 and 25 minutes). When the neuron's response reached this plateau, this was considered the maximal effect of the drug on the neuron and response maps, PSTH and SAM data were collected again during pharmacological manipulation. Following data collection, the drug was again retained in the barrel and the neuron was allowed to recover. Full recovery could be as quick as 30 minutes (bicuculline) or as long as 90 minutes (bicuculline and strychnine cocktail). If the neuron failed to show any signs of recovery after 60 minutes or was lost prior to recovery, we waited at least 30 minutes before searching for another neuron to avoid recording control data from a neuron already demonstrating drug effects.

\section{Localization of recording sites}

Electrode tracts that included recordings from a single unit before and during drug 
application were marked with small deposits of biocytin, applied by iontophoresis (from the recording barrel, $400 \mathrm{nA}$ for ten minutes). For reference and to aid in accurate localization of recording sites, a deposit was also made one or two millimeters dorsal to the recording site in the reticular formation. Upon the completion of a recording session, the animal was given a supplemental dose (2/5 of the original dose) of xylazine and ketamine and perfused through the ascending aorta with a vascular rinse of normal saline $(0.9 \% \mathrm{NaCl})$ followed by a fixative composed of $4 \%$ paraformaldehyde and $0.1 \%$ glutaraldehyde in $0.12 \mathrm{M}$ sodium phosphate buffer. Following perfusion, the brain was dissected from the cranium, post-fixed for at least 24 hours in $4 \%$ paraformaldehyde and $0.1 \%$ glutaraldehyde in $0.12 \mathrm{M}$ sodium phosphate buffer and cryoprotected overnight in $30 \%$ sucrose in $0.12 \mathrm{M}$ sodium phosphate buffer. Brainstems were sectioned on a freezing microtome in the coronal plane at a thickness of $60 \mu \mathrm{m}$. Free-floating sections were incubated for one hour in the avidin-biotin complex (Vector Laboratories, Burlingame, CA) and biocytin was revealed by incubating sections in a solution of $0.05 \%$ diaminobenzidine, $0.01 \%$ hydrogen peroxide, $0.025 \%$ cobalt chloride and $0.02 \%$ nickel ammonium sulfate for two to five minutes. Sections were then mounted onto glass slides and counterstained for Nïssl substance with neutral red.

Drawings of sections containing injection sites were made at a magnification of $41 \mathrm{X}$ using a drawing tube attached to an Olympus microscope. The distance between the two deposits of biocytin was measured and used to calculate tissue shrinkage. This permitted the precise determination of recording site locations along the electrode track, by using depth measurements taken directly from the Burleigh microdrive readout and adjusting for tissue shrinkage. 


\section{RESULTS}

Basic response properties of SPON neurons in rat have been described in the previous chapter (chapter 4). Briefly, SPON neurons discharge at the offset of pure tone and broad band noise stimuli and synchronize to SAM stimuli up to $200 \mathrm{~Hz}$. The offset responses observed in the SPON could be divided into three classes, offset-transient (neurons firing only once per stimulus), offset-choppers (neurons firing two or more well timed spikes) and offset-sustained (neurons with responses that lasted more than twenty ms). Additionally, the vast majority of SPON neurons respond only to contralateral stimulation and have very low rates of spontaneous activity.

To ensure that the effects we observed on neuronal response properties with iontophoretic application of drugs was truly a drug effect and not an effect of current injection or $\mathrm{pH}$ we performed two controls. We recorded from neurons while injecting saline $(0.9 \% \mathrm{NaCl})$ or drug vehicle $(0.165 \mathrm{M} \mathrm{NaCl}, \mathrm{pH} 3)$. We observed no change in the number of spikes or the timing of those spikes with iontophoretic application of saline or vehicle (figure 5.1). Additionally, application of bicuculline and strychnine had drastically different effects on SPON response properties, indicating that bicuculline and strychnine were acting at specific receptors (described below).

\section{Effects of blocking $\mathrm{GABA}_{A}$ input}

We recorded from twelve SPON neurons before, during and after application of the $\mathrm{GABA}_{\mathrm{A}}$ receptor antagonist, bicuculline (BIC). Control PSTHs from six of the neurons were classified as offset-choppers, three as offset-transient and three as offset-sustained. During application of BIC, seven of the twelve neurons (58\%) showed an increase in the total number of spikes during the offset portion of the response, with little or no spike activity during the stimulus (figure 5.2A). During BIC application, five neurons (42\%) responded weakly during the stimulus and at the offset (figure 5.2B). Of the five neurons that displayed spike activity during the stimulus, two had offset transient and three had offset chopper PSTHs in control recordings, indicating that GABAergic inhibition arriving during the stimulus has little effect on 
the classification of the offset response. In all neurons tested $(n=12)$ BIC caused a significant increase $(p<0.05$, ANOVA) in the total number of spikes in response to a $50 \mathrm{~ms}$ pure tone (figure 5.3). The increase in the total number of spikes for all twelve neurons ranged from 24.4 $\%$ to $417.4 \%$ and averaged $129.1 \%$. The average increase in the total number of spikes for the seven neurons that had only offset discharges averaged (average \pm SD) $92.42 \pm 79.41 \%$, and was $178.2 \pm 152.91 \%$ for the five neurons that responded during the stimulus and at the offset.

Spontaneous rates for the population of twelve SPON neurons before drug delivery averaged $1.0 \pm 2.13$ spikes per second. During application of BIC, four neurons showed an increase in spontaneous activity and two showed a decrease. Overall, the average spontaneous rate in the presence of BIC was still very low, measuring $1.16 \pm 1.33$ spikes per second and was not significantly different from control ( $p=0.38$, paired $t$ test).

\section{Effects of blocking glycinergic input}

We tested the responses of twelve SPON neurons with application of the glycine receptor antagonist, strychnine (STRYCH). Control PSTHs from six of these neurons were classified as offset-chopper, four as offset-transient and two as offset-sustained. During STRYCH application, the offset response was abolished in all twelve neurons and transient responses timed to the stimulus onset were observed (figure 5.4), indicating that the offset response depends on glycinergic input. In the presence of STRYCH, first spike latencies averaged $13.07 \pm 2.86 \mathrm{~ms}$ from the tone onset. The effect of STRYCH on the total number of spikes in response to a $50 \mathrm{~ms}$ tone burst was variable (figure 5.5). Seven of twelve neurons (58\%) showed a significant increase $(p<0.006$, ANOVA) in the total number of spikes; three neurons $(25 \%$; 1 offsetchoppers, 1 offset-transient, 1 offset-sustained) showed a significant decrease $(p<0.001$, ANOVA) in the total number of spikes and two neurons showed no change in the total number of spikes ( $p>0.05$, ANOVA) in response to a $50 \mathrm{~ms}$ tone burst.

The effects of STRYCH on the number of spontaneous discharges was variable. For this population of twelve neurons the rate of spontaneous discharges in the control condition averaged $3.75 \pm 6.79$ spikes per second; in the presence of STRYCH, spontaneous rates averaged 
$4.5 \pm 8.83$ spikes per second and this difference was not significant ( $p=0.39$, paired $t$ test).

Response maps from ten neurons were collected before and during application of STRYCH (figure 5.6). BFs for this population ranged from 2.3 to $23.4 \mathrm{kHz}$. SPON response maps are typically $\mathrm{V}$ shaped with narrow regions near BF and long low frequency receptive areas. Overall, blockage of glycinergic inhibition resulted in response maps that still had narrow peaks and low frequency tails but were generally broader with responses to frequencies that were outside the original receptive field. We quantified the width of the receptive fields at $20 \mathrm{~dB}$ above the threshold by calculating $\mathrm{Q}_{20}$ values. $\mathrm{Q}_{20}$ values for control response maps averaged $3.07 \pm 1.82$ compared to $1.55 \pm 1.05$ in the presence of STRYCH and this difference was significant $(p<0.05$, paired $t$ test).

\section{Effects of blocking $\mathrm{GABA}_{A}$ and glycine inputs simultaneously}

A total of fourteen neurons were tested with a cocktail of BIC and STRYCH. Control PSTHs from nine of these neurons were classified as offset-transient, three as offset-chopper and two as on-offset. The PSTHS during drug application in all cases were unlike those recorded in the presence of BIC or STRYCH alone, providing evidence that both drugs were acting on target receptors. Application of the BIC/STRYCH cocktail abolished the offset response and resulted in long sustained responses timed to the stimulus onset (figure 5.7). In five neurons (35\%) the PSTHs during BIC/STRYCH showed "chopper" like responses, characterized by three or more

regularly timed peaks resembling multipolar neurons in the cochlear nucleus (figure 5.7B). In five neurons (36\%) the response lasted longer than the stimulus (figure 5.7A). Each of these neurons had an excitatory response that overlapped the offset activity observed in the control condition. Of the control PSTHs from these five neurons with sustained excitation that overlapped the inhibitory rebound, three had onset-transient and two had offset-chopper responses, indicating that this persistant excitatory input does not contribute to the type of offset response observed in control recordings. During blockade of GABA and glycine the first spike latency averaged $13.56 \pm 3.13 \mathrm{~ms}$ from the stimulus onset. As expected, all neurons showed a significant increase ( $p<0.05$, ANOVA) in the total number of spikes in response to a $50 \mathrm{~ms} \mathrm{BF}$ tone burst (figure 5.8). The increase in number of spikes ranged from $124 \%$ to $2160 \%$ and 
averaged $516 \%$.

For the population of fourteen neurons tested with the bicuculline and strychnine cocktail, the number of spontaneous discharges before drug application averaged $0.78 \pm 1.18$ spikes per second and averaged $10.35 \pm 14.67$ spikes per second during application of BIC/STRYCH. The difference in spontaneous rates was significant between the two conditions ( $p<0.015$, paired $t$ test). Of the fourteen neurons tested, eleven had an increase in the number of spontaneous discharges, two neurons showed no change and one neurons had a decrease in the number spontaneous discharges in the presence of BIC/STRYCH.

Response maps were collected for eleven neurons before and during application of $\mathrm{BIC/STRYCH}$. BFs for these neurons ranged from 3.8 to $22.3 \mathrm{kHz}$. As previously described, SPON response maps are $\mathrm{V}$ shaped with narrow peaks near BF and low frequency tails. Generally, control response maps were more narrow than response maps collected during application of BIC/STRYCH as quantified with $\mathrm{Q}_{20}$ values (figure 5.9). In all cases except one (92\%), we noticed broader receptive fields and lower $\mathrm{Q}_{20}$ values when $\mathrm{GABA}_{\mathrm{A}}$ and glycine receptors were blocked. Control $\mathrm{Q}_{20}$ values averaged $3.91 \pm 2.3$ and BIC/STRYCH $\mathrm{Q}_{20} \mathrm{~S}$ averaged $2.54 \pm 1.96$ and this difference was statistically significant $(p<0.015$, paired $t$ test; figure 5.10). Thresholds were not significantly different between control and drug conditions (29.08 \pm 15.56 and $29.77 \pm 15.48$ respectively, $p=0.35$, paired $t$ test).

Twelve neurons were tested with SAM pure tones before and during application of $\mathrm{BIC/STRYCH}$; of the twelve neurons tested one neuron was inhibited for the entire $500 \mathrm{~ms}$ presentation and was considered unresponsive to SAM. SPON neurons typically synchronize to the envelope of SAM stimuli with an upper cutoff between 100 and $200 \mathrm{~Hz}$. When $\mathrm{GABA}_{\mathrm{A}}$ and glycine receptors were blocked, phase locking capabilities were reduced and poorly timed sustained responses were typically observed (figure 5.11). Synchrony of the response to the modulation frequency (50 to $800 \mathrm{~Hz}$ ) was quantified by calculating vector strength for the eleven neurons that synchronized SAM stimuli. Vector strengths for the population of eleven neurons that responded to SAM are shown in figure 5.12. When inhibition was blocked, there was a significant decrease $(p<0.01$, paired $t$ test) in the vector strength at all modulation frequencies 
tested. 


\section{DISCUSSION}

We have shown that GABAergic and glycinergic inhibition exert a powerful influence on the response properties of SPON neurons and are essential for the formation of the offset response. Blocking GABAergic and glycinergic inhibition separately had very different effects on response properties of SPON neurons, a phenomenon that has been described in the mustache bat dorsal nucleus of the lateral lemniscus (Yang and Pollak, 1994). The effects of blockade of the GABA and glycine receptors will be discussed separately below.

\section{Blockade of the $G A B A_{A}$ receptor}

Blocking the $\mathrm{GABA}_{\mathrm{A}}$ receptor with bicuculline resulted in an increase in the number of offset spikes fired in response to a stimulus. We interpret this data to mean that SPON neurons receive a GABAergic inhibition that is timed to the stimulus offset. The GABAergic inhibition timed to the stimulus offset likely arises from other SPON neurons. Indeed, our previous tract tracing and intracellular labeling studies suggest that SPON axons provide intrinsic collaterals that terminate within the nucleus and our pharmacology data is consistent with this observation (Kulesza et al., 1999; Saldaña and Berrebi, 2000). In some neurons, blocking the $\mathrm{GABA}_{\mathrm{A}}$ receptor revealed spike activity during the stimulus presentation, indicating a second GABAergic input may impinge on these cells during the stimulus. A potential source of this input are the neurons of tecto-commissural column, situated within the commissure of the inferior colliculus (Viñuela and Saldaña, 2000).

\section{Blockade of the glycine receptor}

Blocking the glycine receptor with strychnine had variable effects on the number of spikes fired in response to a stimulus but did have a major effect on the timing of the spikes. Strychnine abolished the offset response in all neurons tested and we never observed the offset activity seen in control recordings. During strychnine application, SPON neurons responded during the stimulus with first spikes timed to the stimulus onset. The fact that offset responses could be abolished by blocking glycinergic inhibition, indicates that the offset response results 
from a postinhibitory rebound mechanism (see below).

\section{Blockade of both $\mathrm{GABA}_{A}$ and glycine receptors}

Blockage of both the $\mathrm{GABA}_{\mathrm{A}}$ and glycine receptors simultaneously, resulted in a dramatic effect on the number of spikes and the timing of those spikes in response to a $50 \mathrm{~ms}$ pure tone stimulus. During application of bicuculline and strychnine, the offset response elicited by SPON neurons was completely abolished and neurons instead fired in a sustained discharge pattern for the duration of the stimulus. In nearly half of SPON neurons, the sustained discharge outlasted the stimulus such that excitatory activity was observed in the time window where offset activity was evoked in the control condition (figure 5.7A). Thus, for nearly half of our SPON neurons we cannot rule out the possibility that inhibitory rebound coincides with excitatory input and that both inhibitory rebound and excitatory input contribute to the offset response.

It has been established that the main excitatory input to the SPON arises from the octopus and multipolar cells in the contralateral cochlear nucleus (Friauf and Ostwald, 1988; Schofield, 1995). In the fourteen neurons studied with BIC/STRYCH, seven neurons (65\%) had pure tone PSTH patterns during drug application (presumably representing purely excitatory input) that did not resemble responses described for octopus or multipolar cells in the ventral cochlear nucleus (Godfrey et al., 1975; Rhode et al., 1983). Interestingly, five neurons (35\%) had PSTH patterns with regularly spaced peaks and resembled the "chopper" pattern that has been described for multipolar neurons in the ventral cochlear nucleus (Rhode et al., 1983). The PSTH pattern observed in these five neurons supports evidence that SPON neurons are driven by multipolar cells. Indeed, the sustained responses of SPON neurons (in the absence of inhibition) provide evidence that all SPON neurons receive multipolar cell input. However, since most SPON neurons had sustained responses (when inhibition was blocked) with no noticeable onset features, it is difficult to assess the contribution of onset excitation from octopus cells to SPON physiology.

Our bicuculline data provide evidence that less than half of SPON neurons receive a GABAergic input that arrives during the stimulus. Interestingly, we observed many more spikes 
with BIC/STRYCH than with STRYCH alone. A potential explaination for the large increase in spike activity when both $\mathrm{GABA}_{\mathrm{A}}$ and glycine receptors are blocked is that a sustained GABAergic input reaches most if not all SPON neurons during the stimulus. The reason only a few neurons showed excitatory responses during the stimulus when only $\mathrm{GABA}_{\mathrm{A}}$ receptors are blocked, may be that glycinergic inhibition alone is strong enough to fully inhibit most neurons during the stimulus. Thus, it appears that there are at least two distinct GABAergic inputs that innervate most if not all SPON neurons, one that arrives during the stimulus and one coincident with the offset.

\section{General effects of inhibition on SPON neurons}

Blocking glycinergic inhibitory input to SPON neurons broadened response maps, a feature that has been described for inferior colliculus neurons (Yang et al., 1992). Response maps of SPON neurons, which reflect rebound from glycinergic inhibition, are wider than previously published response maps from cat MNTB neurons (Guinan, 1968; Kulesza et al., submitted). Since offset response maps are dictated by glycinergic input and SPON response maps are wider than those in the MNTB, the MNTB must converge onto SPON neurons. Our response map data during pharmacological manipulation shows that the excitatory input reaching the SPON from the contralateral cochlear nucleus is more broadly tuned than the glycinergic input from the MNTB. A broadly tuned excitatory innervation from the cochlear nucleus might arise from the broadly tuned octopus cells or convergence of multipolar cell inputs (Godfrey et al., 1975; Rhode and Smith, 1986). If the excitatory input is more broadly tuned than the glycinergic input causing rebound, what prevents SPON neurons from responding to the excitatory input that arrives outside the typical V shaped receptive field in the control condition? Our data show that receptive fields of SPON neurons were broadened during blockade of the glycine receptor. We interpret this data to indicate that a glycinergic input, also likely arising from the MNTB but insufficient to cause inhibitory rebound innervates the SPON and prevents the neurons from responding to the broadly tuned excitatory input. If this is the case, it provides evidence that there is some degree of converging and partial overlapping of MNTB inputs onto SPON neurons. 
SPON responses in the presence of BIC/STRYCH display a rather long first spike latency, nearly $14 \mathrm{~ms}$ from the stimulus onset, compared to our own MNTB recordings where first spike latency averaged $6.27 \mathrm{~ms}$ from the stimulus onset. This is an interesting feature given the known input from octopus and multipolar cells, which should arrive with a short latency. Octopus cells have short first spike latencies ( $3 \mathrm{~ms}$ from the stimulus onset) and also innervate neurons in the ventral nucleus of the lateral lemniscus (Adams, 1997). First spike latencies in VNLL neurons average $7.0 \pm 1.4 \mathrm{~ms}$ (Batra and Fitzpatrick, 1999). Thus, there is a large discrepancy in first spike latencies between the SPON and VNLL, both innervated by octopus cells. A possible reason for this discrepancy in first spike latency might be the arrangement of the excitatory and inhibitory inputs on SPON neurons. Ultrastructural analysis suggests that nearly $70 \%$ of the synapses on SPON somata are inhibitory (GABAergic or glycinergic) while nearly $70 \%$ of the synapses on dendritic profiles are excitatory (Holt and Berrebi, 1999). Additionally, some ventrally directed dendrites of SPON neurons extend up to $430 \mu \mathrm{m}$ away from the soma (Saldaña and Berrebi, 2000). Therefore, much of the excitatory input reaching the SPON arrives some distance from the soma. Consequently, excitatory inputs arriving mainly on the dendrites and far from the soma, may only weakly influence firing properties and the temporal precision of these excitatory inputs may be lost by the time the neurons can fire an action potential. In a population of our own recordings from MNTB neurons, the first spike latency averaged $6.27 \pm 1.34 \mathrm{~ms}$ and first spike latency of excitatory input to the SPON averaged $13.56 \pm 3.13 \mathrm{~ms}$. MNTB axons travel approximately $300-400 \mu \mathrm{m}$ to targets in the SPON (personal observations); assuming an axonal conduction velocity of 5 meters per second and a synaptic delay of a few ms, inhibitory input from the MNTB reaches the SPON about $7 \mathrm{~ms}$ from the sound onset, long before the SPON can respond to excitatory inputs. Thus it appears, that the differences in the arrival time of excitatory and inhibitory inputs are an important component of the SPON circuit contributing to the formation of the offset response.

\section{Response of SPON units to SAM}

The ability of SPON neurons to phase lock to SAM stimuli appears to be mediated largely by the inhibitory input to this cell group. In the inferior colliculus, inhibition has been shown to expand the range of amplitude modulation neurons were capable of synchronizing to 
and to reduce vector strengths (Koch and Grothe, 1998). In most of our SPON neurons, removing inhibition abolished or greatly reduced the neurons ability to phase lock to amplitude modulated stimuli. The weak responses to SAM in the absence of inhibitory input indicates that the excitatory inputs to the SPON respond weakly or not at all to SAM stimuli. Responses to SAM stimuli have been recorded from neurons in the cat and gerbil cochlear nuclei (Rhode and Greenburg, 1994; Frisina et al., 1990). Onset neurons (presumed to represent octopus cells) and choppers (presumed to represent multipolar cells) synchronize to SAM up to 1000 and $600 \mathrm{~Hz}$ respectively. Thus, these inputs should provide excitatory, phase locked input to the SPON. Interestingly, in both cat and gerbil, the ability of cochlear nucleus neurons to follow SAM at high intensity (60 to $90 \mathrm{~dB}$ above threshold) is reduced (Rhode and Greenburg, 1994; Frisina et al., 1990). The average threshold for the twelve SPON neurons tested with SAM was $29.08 \pm$ 15.56 dB SPL and SAM stimuli were presented at $20 \mathrm{~dB}$ above threshold. Thresholds for onset and chopper neurons in the cat cochlear nucleus are about $19 \mathrm{~dB}$ (Rhode and Smith, 1986) and our stimuli were presented at about $30 \mathrm{~dB}$ above octopus and multipolar cell thresholds. Thus, one possible reason for the diminished SAM sensitivity of SPON neurons when both $\mathrm{GABA}_{\mathrm{A}}$ and glycine were blocked is the high intensity of the stimulus ( $50 \mathrm{~dB}$ SPL). At this high intensity level, the responses of cochlear nucleus neurons to SAM stimuli may begin to lose temporal precision and this would be reflected in the poorly timed response of SPON neurons when inhibition is blocked. Another possible reason for the lack of SAM sensitivity in the presence of BIC/STRYCH is the fact that most excitation is arriving on the dendrites and this may act to smear any temporal acuity in the input.

\section{Mechanism of the offset response}

The generation of offset responses by post inhibitory rebound (PIR) is an interesting feature of the nervous system and provides a mechanism where one input can provide both inhibition and excitation. Our data shows the offset response is abolished when glycinergic input is blocked, indicating that it is a rebound from inhibition and rules out a descending or long latency excitatory input as the source of offset activity. In some neurons, when GABA and glycine were blocked, the excitatory response outlasted the stimulus with a time course that even outlasted the offset response. Thus, in some neurons, postinhibitory rebound (PIR) coincident 
with excitatory input may be required for the offset response. The mechanism that leads to PIR is unclear. However, in slice preparations PIR can be induced by a hyperpolarizing current pulse and has been demonstrated in vivo by application of GABA (Aizenman and Linden, 1999; Plenz and Kital, 1999). Neurons displaying PIR have been reported in the rat IC and PIR was blocked with TTX, a blocker of sodium channels, and in low calcium solutions, providing evidence that calcium and sodium currents contribute to PIR (Sivaramakrishnan and Oliver, 2001). PIR is believed to be triggered by the opening of $\mathrm{T}$ type (low voltage activated) calcium channels (Aizenman and Linden, 1999). These channels are deinactivated during hyperpolarization of the cell membrane and open on return to more depolarized membrane potentials, resulting in a large influx of calcium (Aizenman and Linden, 1999). The calcium influx presumably leads to the depolarization that may lead to opening of deinactivated sodium channels resulting in sodium spikes. Another potential contributer to PIR is the Ih current (McCormick and Pape, 1990). Ih is a mixed cation current that is activated during hyperpolarization and drives the membrane potential to more depolarized levels and has been implicated in postinhibitory rebound spikes (Aizenman and Linden, 1999).

\section{Conclusions}

Our pharmacological data provide solid evidence that offset responses, at least in the SPON, result from a postinhibitory rebound mechanism caused by removal of MNTB-mediated glycinergic inhibition at the end of a sound stimulus. Synchronized responses to SAM stimuli are largely mediated by inhibition. Our data also provide evidence that normal response features of SPON neurons (offset responses, phase locking to SAM, receptive fields) are created through an integration of membrane properties and glutamatergic, GABAergic and glycinergic innervation. Additionally, our data may provide some useful insight into how the arrangement of inputs on a cell can affect neuronal response properties that may be applicable to other neuronal circuits.

\section{Future direction}

Although we have examined the role of $\mathrm{GABA}_{\mathrm{A}}$ and glycine receptors in SPON response 
properties, some important questions remain. We have used bicuculline to block the $\mathrm{GABA}_{\mathrm{A}}$ receptor, but $\mathrm{GABA}$ is also acting on the metabotropic $\mathrm{GABA}_{\mathrm{B}}$ receptors. The contribution of the $\mathrm{GABA}_{\mathrm{B}}$ receptor to the physiological response properties of SPON neurons could be examined with recordings made in the presence of the $\mathrm{GABA}_{\mathrm{B}}$ antagonist saclofen (Kerr et al., 1989). Additionally, it remains unclear if the offset response requires an excitatory input along with the glycinergic inhibition. The role of excitatory, glutamatergic input could be studied by iontophoretic application of the non-selective glutamate receptor antagonist, kynurenic acid (Perkins and Stone, 1982). Preliminary data from two SPON neurons before, during and after kynurenic acid application provide evidence that glutamatergic input is not required for the offset response and further supports a post-inhibitory rebound mechanism. 


\section{Acknowledgments}

I would like to thank Dr. Jeffrey Wenstrup for instruction on making and using the "piggy back" electrodes. I would also like to thank Brian Pope for assistance with tissue processing and Jesse Thompson for his help with Matlab. 
A
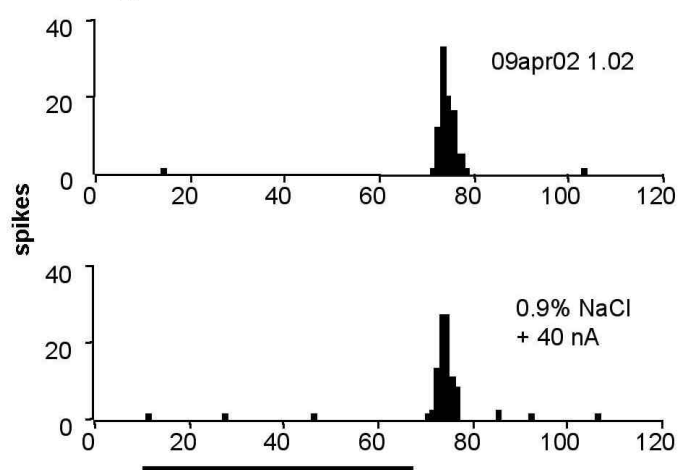

B
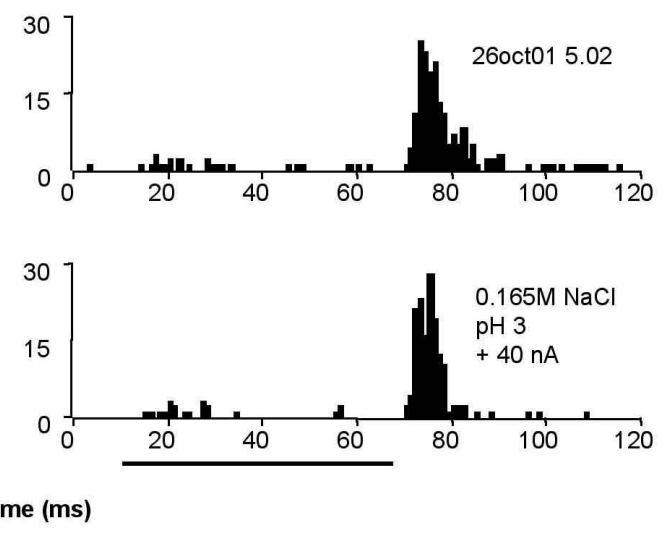

Figure 5.1 Offset responses in SPON neurons were unaffected by extracellular current injection and vehicle $\mathrm{pH}$. In order to demonstrate a specific action of pharmacological manipulations, we recorded from neurons while iontophoretically delivering normal saline (A) or drug vehicle (B) with relatively high currents. This did not affect neuronal discharges. 
A
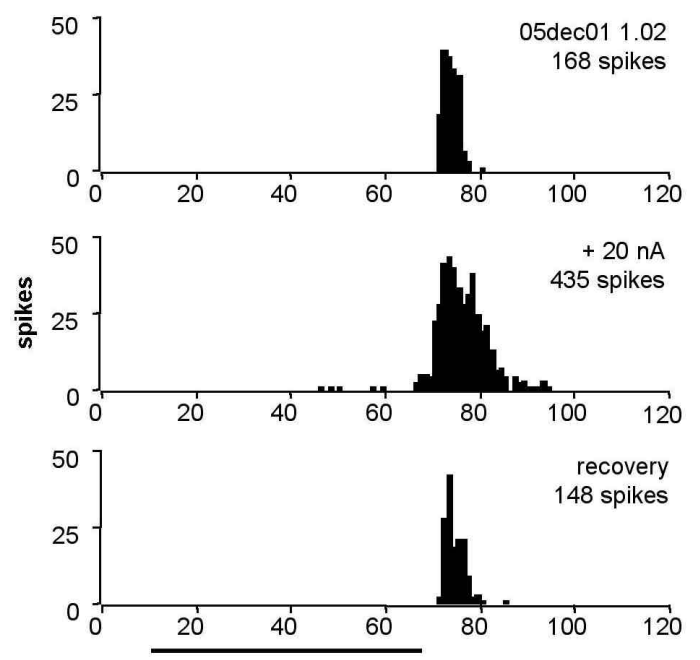

B
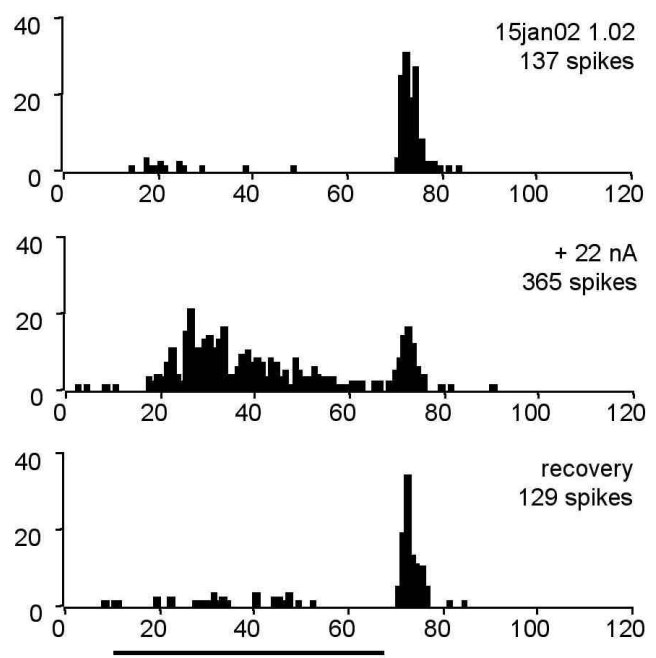

ime (ms)

Figure 5.2. Blockage of the $\mathrm{GABA}_{\mathrm{A}}$ receptor caused an increase in the number of offset spikes and had little effect on temporal discharge pattern. In most neurons (7/12), blockage of the $\mathrm{GABA}_{\mathrm{A}}$ receptor did not change the temporal discharge patterns of SPON neurons, but did cause an increase in the number of evoked spikes at the offset (A). In a few neurons (5/12), blockage of the $\mathrm{GABA}_{\mathrm{A}}$ receptor resulted in spike activity during the stimulus and at the offset (B). 


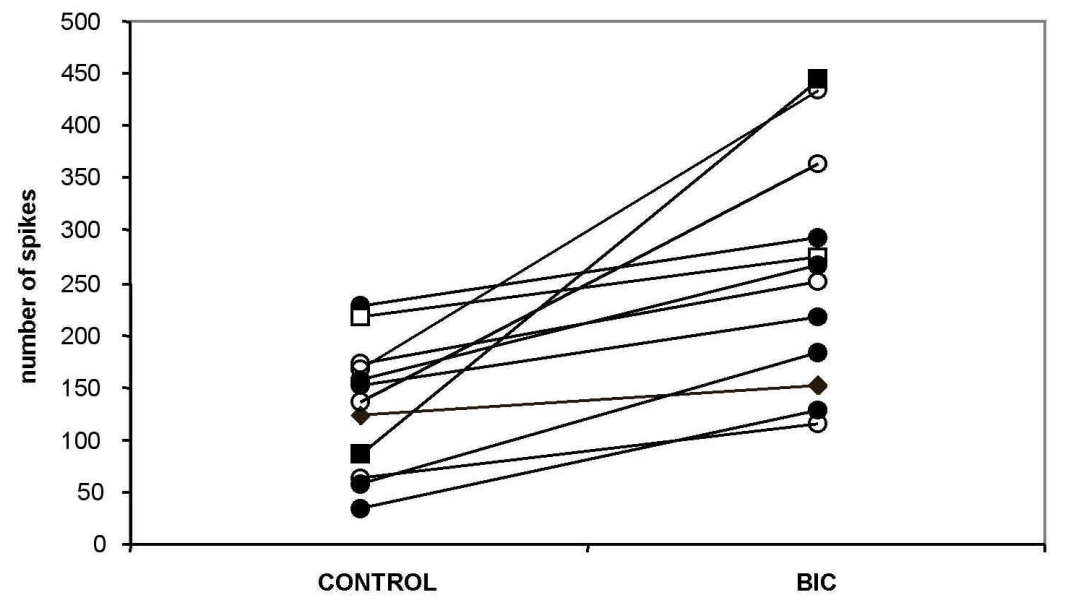

Figure 5.3. Blockage of the $\mathrm{GABA}_{\mathrm{A}}$ receptor caused an increase in the number of evoked spikes. In all neurons tested, bicuculline caused a significant increase in the number of spikes evoked by a $50 \mathrm{~ms}$ stimulus (100 sweeps). In some cases there was a $200 \%$ increase in the number of spikes over the control. 

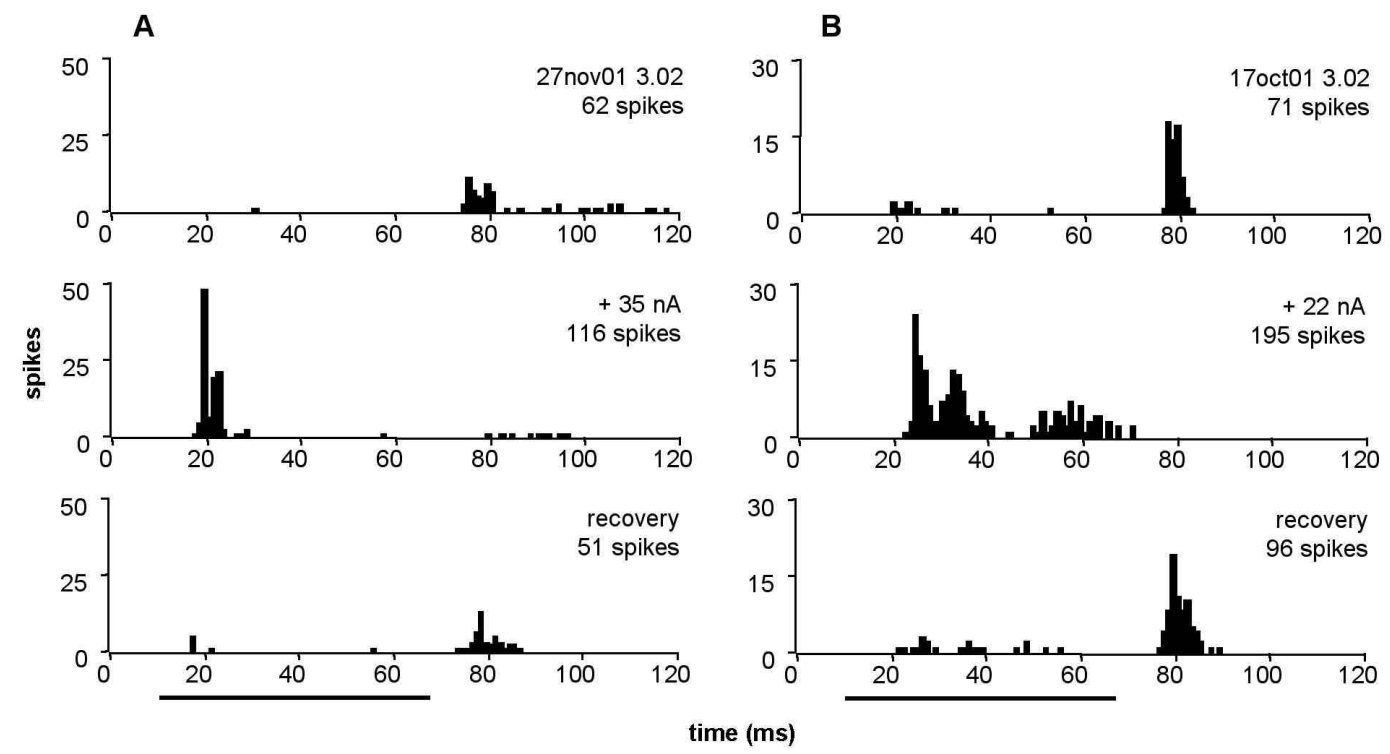

Figure 5.4. Blocking the glycine receptor abolished the offset response of SPON neurons. In all neurons tested, strychnine abolished the offset response and activity timed to the stimulus onset was observed. The resulting onset activity could be transient (A) or more sustained (B). 


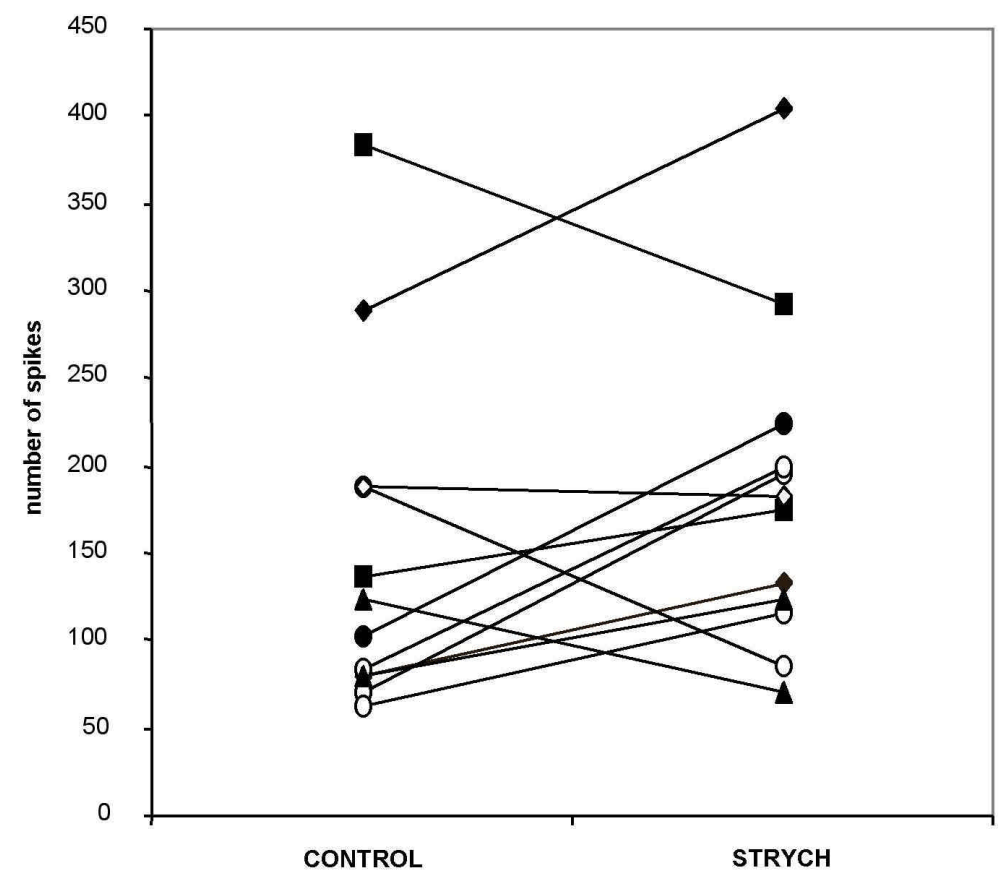

Figure 5.5. Blocking the glycine receptor had a variable effect on the number of evoked spikes. In most neurons tested, strychnine caused an increase in the number of spikes discharged in response to a $50 \mathrm{~ms}$ stimulus. Approximately one-third of the neurons tested with strychnine showed a decrease in the total number of spikes. 

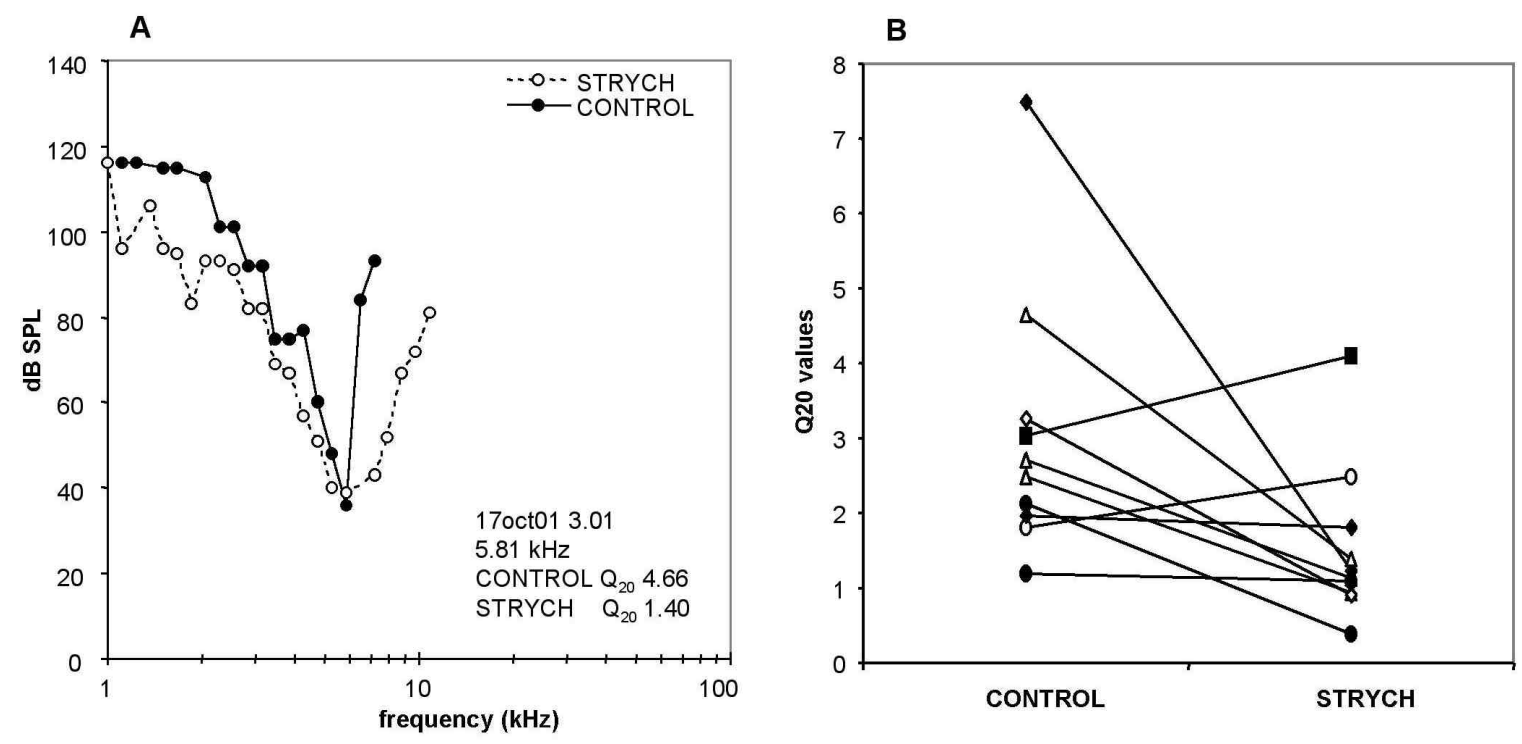

Figure 5.6. Blocking the glycine receptor broadened response maps. Response maps collected during strychnine application were generally broader than the offset response maps recorded with control data (A). Overall, $\mathrm{Q}_{20}$ values decreased significantly, indicating broader receptive fields, in the presence of strychnine (B). 
A
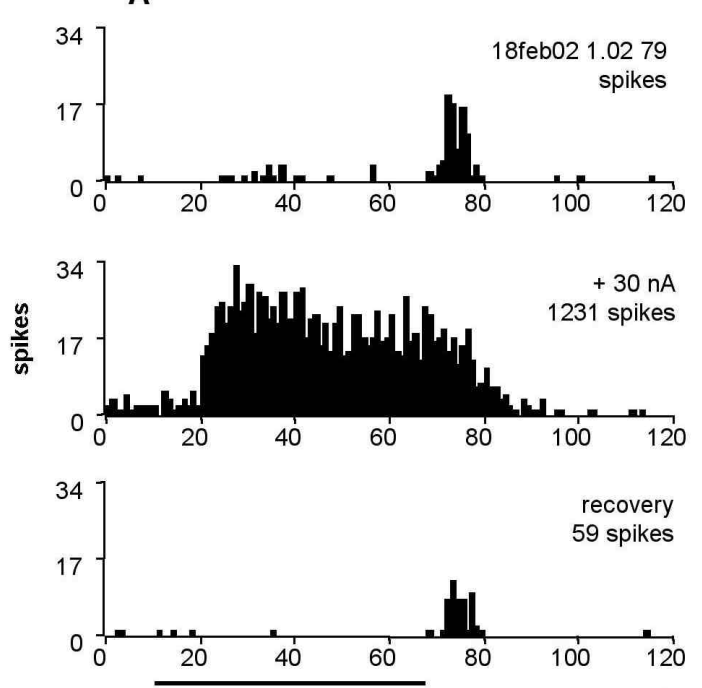
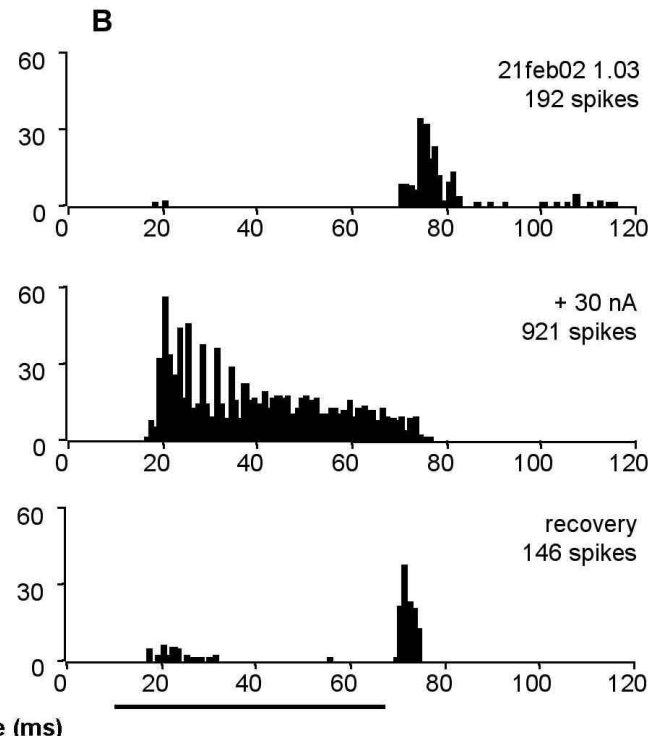

Figure 5.7. Blockage of the $\mathrm{GABA}_{\mathrm{A}}$ and glycine receptors together changed temporal discharge pattern and the number of evoked spikes. In all neurons tested, BIC/STRYCH abolished the offset and resulted in sustained discharges that could outlast the stimulus such that excitatory activity occurred in place of the offset response (A). Some neurons had chopper like PSTHs (B) 


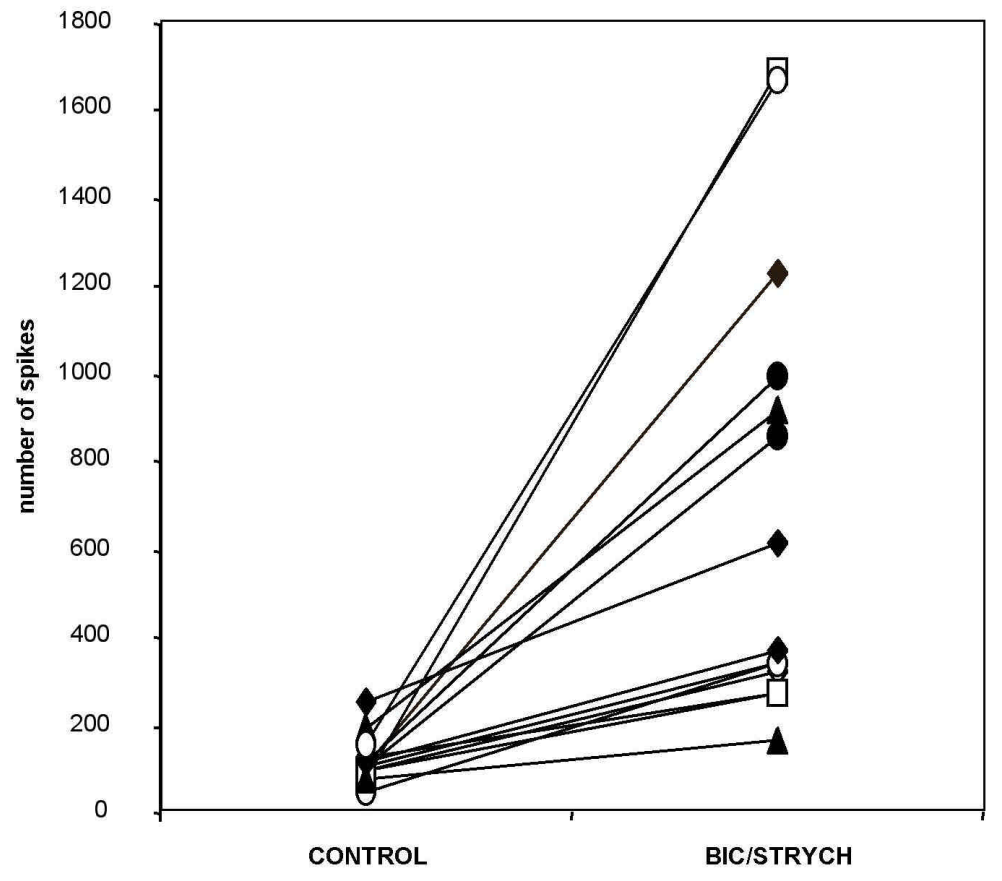

Figure 5.8. Blockage of the $\mathrm{GABA}_{\mathrm{A}}$ and glycine receptors together greatly increased the number of evoked spikes. In all neurons tested with bicuculline and strychnine we observed a significant increase in the number of spikes. In half of the neurons tested (6 of 12), there was a greater than five-fold increase in the number of spikes to a $50 \mathrm{~ms}$ stimulus. 


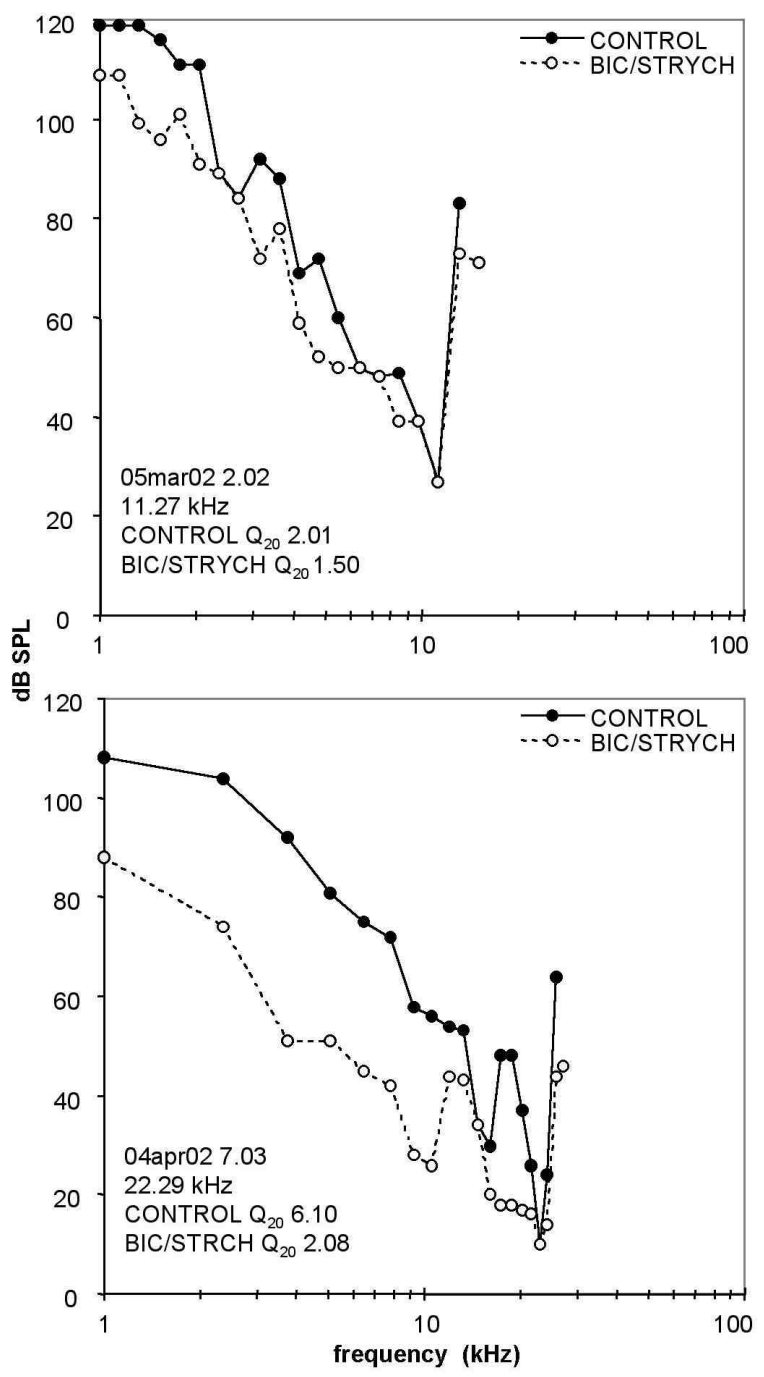

Figure 5.9. Blockage of the $\mathrm{GABA}_{\mathrm{A}}$ and glycine receptors together broadened response maps. Response maps are shown for two neurons before and during application of bicuculline and strychnine. For the neuron in A, the response maps from the control and drug condition are similar near BF but when inhibitory input is blocked, the neuron becomes more sensitive on the low frequency side of the response map. In $\mathrm{B}$, there is a noticeable broadening near BF when inhibition is removed and the neuron has a much lower threshold to low frequency tones (1 to 10 $\mathrm{kHz})$. 


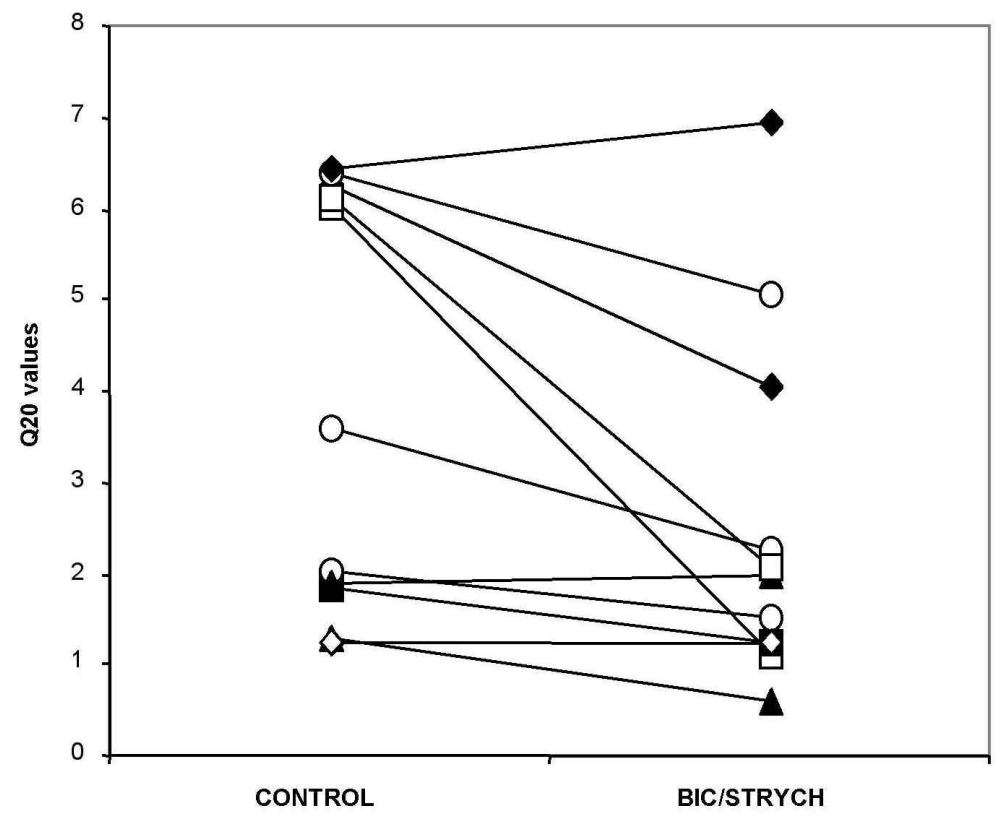

Figure 5.10. Blockage of the $\mathrm{GABA}_{\mathrm{A}}$ and glycine receptors together resulted in lower $\mathrm{Q}_{20}$ values. $\mathrm{Q}_{20}$ values for the eleven neurons before and during bicuculline and strychnine are compared. Overall, there was a significant decrease in $\mathrm{Q}$ values when inhibition was blocked. 


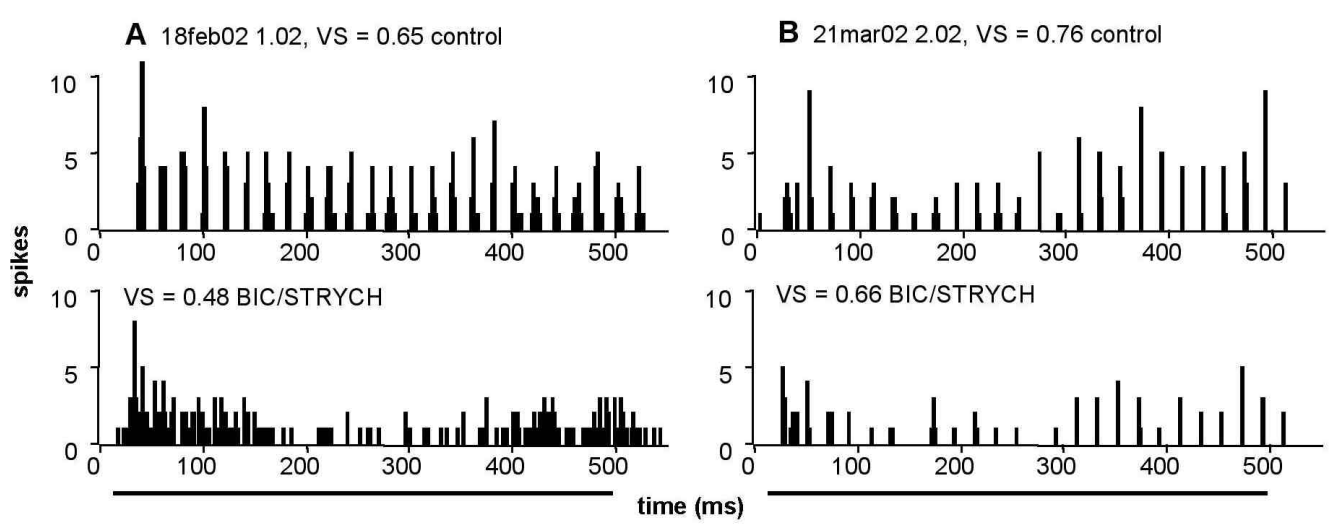

Figure 5.11. Blockage of the $\mathrm{GABA}_{\mathrm{A}}$ and glycine receptors together reduced phase locking capabilities to SAM stimuli. Shown are responses to a $50 \mathrm{~Hz}$ modulated $\mathrm{BF}$ tone, $20 \mathrm{~dB}$ above threshold from two neurons before and during application of bicuculline and strychnine. Before drug application the neuron in A responded to each modulation cycle with a single spike; during drug application the cell failed to show any phase locking to SAM. In B, some degree of phase locking is retained during bicuculline and strychnine application. 


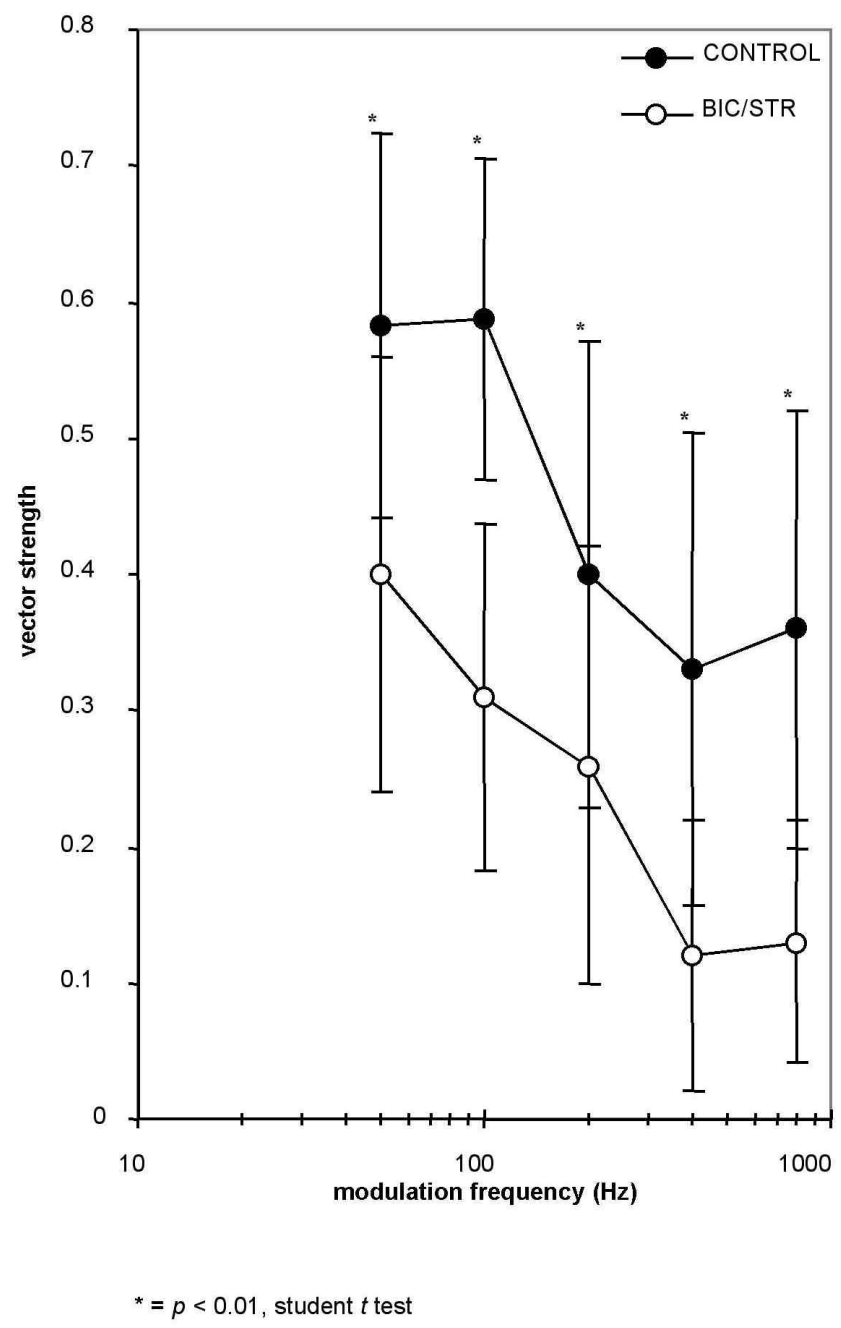

Figure 5.12. Blockage of the $\mathrm{GABA}_{\mathrm{A}}$ and glycine receptors together caused a reduction in vector strengths. Average vector strengths from the ten neurons that responded to SAM stimuli for 50,100, 200, 400 and $800 \mathrm{~Hz}$ SAM pure tones before and during drug application are shown. There was a significant decrease in the vector strength at all modulation frequencies tested when inhibition was removed ( $p<0.01$, student $t$ test). 
Chapter Six

General Discussion 


\section{SUMMARY}

The purpose of this dissertation was to characterize SPON neurons in the rat using anatomical and physiological techniques, with the hopes of clarifying the functional role of this cell group. The literature, which is based on data from numerous species, provides evidence that the SPON contains a morphologically heterogeneous population of neurons whose inputs, projections and responses to sound vary with species. The sparse amount of published data from different species makes hypothesizing a functional role for the SPON difficult. Understanding basic features of the SPON such as neurochemical phenotype, connectivity and responses to sound will lead to a testable hypothesis of the functional role of this cell group and clarify the role of inhibitory circuits in hearing. Further, an in depth study of the SPON is required in a single species before generalities about this region of the superior olivary complex can be made. To that end, the data included herein provides the first in depth study of the neurochemistry and physiology of the SPON in any species.

\section{Study I: SPON neurons are GABAergic}

The immunocytochemical investigation of the presence of the inhibitory neurotransmitters, glycine, GABA and the GABA synthetic enzyme GAD presented in chapter 2, indicates that nearly $91 \%$ of SPON neurons are GAD immunoreactive and therefore GABAergic. Additionally, our data indicates that SPON neurons receive abundant glycinergic and GABAergic inhibitory input. Analysis of neuronal morphology indicates that the rat SPON contains a morphologically homogeneous population of neurons, which correlates with published tract tracing data (Saldaña and Berrebi, 2000). SPON somata are multipolar and are elongated rosto-caudally with dendritic trees that are flattened parasagittally.

\section{Study II: The SPON is a major source of inhibition to the IC}

The VNLL, DNLL, SPON and LSO are all sources of inhibitory projections to the inferior colliculus (González-Hernández et al., 1996). Our unbiased stereological estimates of neuronal number, in chapter three, indicate that the rat SPON contains approximately 2,400 
neurons, $91 \%$ of which are GABAergic (Kulesza and Berrebi, 2000). We estimate that the VNLL contains approximately 13,800 neurons, two-thirds of which are inhibitory (Riquelme et al., 2001). Therefore, the VNLL contains about 9,000 inhibitory neurons that project to the IC. The DNLL, often considered the major source of inhibition to the IC, contains approximately 1,800 neurons, all of which are reportedly inhibitory (Adams and Mugnaini, 1984; Zhang et al., 1998). The LSO contains 2,500 neurons, approximately $20 \%$ of which are inhibitory (Mugnaini and Oertel, 1985). Our stereological estimates provide evidence that the VNLL is the major source of ascending inhibition to the IC. The SPON contains more inhibitory neurons than the DNLL and LSO, and is therefore a major source of ascending inhibitory input to the IC.

\section{Study III: SPON neurons respond at the sound offset and can encode sound envelopes}

In chapter four, we present data from in vivo extracellular recordings from SPON

neurons. These data indicate that the vast majority of SPON neurons are monaural, responding only to contralateral stimuli. SPON neurons respond with only a few spikes at the offset of pure tone and broad band noise stimuli and synchronize to amplitude fluctuations in complex stimuli. Nearly half of SPON neurons, mainly offset-sustained and offset-choppers, were sensitive to stimulus duration and these neurons may function in duration tuning. Additionally, mapping of neurons best frequency provides evidence for a tonotopic representation of frequency in the SPON.

\section{Study IV: SPON response properties are formed largely by inhibitory input}

In chapter five, we examined the effects of the inhibitory neurotransmitters GABA and glycine on in vivo response properties of SPON neurons using pharmacological manipulations to block $\mathrm{GABA}_{\mathrm{A}}$ and glycine receptors. In most neurons, blocking GABAergic input resulted in an increase in the number of spikes occurring at the stimulus offset, supporting the existence of intrinsic collateral input from other SPON neurons. Offset responses were abolished by iontophoretic application of the glycine receptor antagonist strychnine, providing evidence that offset responses arise from a postinhibitory rebound mechanism. Blocking both $\mathrm{GABA}_{\mathrm{A}}$ and glycine receptors resulted in broadened response maps and poorly timed responses to amplitude 
modulated stimuli. Thus, inhibitory input plays an important role in SPON physiology and is essential for normal response characteristics of SPON neurons.

The data contained in this dissertation, combined with the available literature, indicate the rat SPON is a prominent source of GABAergic, offset inhibition to the IC. This work has led us to hypothesize that the SPON is involved in processing temporal features of sound, specifically the offset and amplitude fluctuations. Inhibitory inputs to the IC have been shown to be involved in many aspects of auditory processing. However, the role of offset inhibition is likely limited to a few aspects of processing in the inferior colliculus. The possible functional role that offset inhibition might play in the IC will be the focus of the following section.

\section{DISCUSSION}

\section{Model for the formation of offset responses}

Our physiology and pharmacology data is consistent with the offset response arising from a postinhibitory rebound mechanism. Offset responses were abolished by blockade of glycine receptors, providing evidence that offset activity is dependent on MNTB input. Thus, response maps of SPON neurons reflect glycinergic input and comparing Q values suggests that SPON units have wider receptive fields than MNTB neurons. We interpret this to mean that there is some degree of convergence of MNTB input onto SPON neurons. Responses to excitatory input from the contralateral $\mathrm{CN}$ in the presence of $\mathrm{GABA}_{\mathrm{A}}$ and glycine receptor antagonists, come at considerable delay relative to the stimulus onset. This long latency is likely a result of the distribution of excitatory inputs on the dendrites of SPON neurons and the location of excitatory inputs distally may serve to attenuate excitatory drive to the cell. Generation of offset spikes also likely requires a particular complement of membrane channels, specifically $\mathrm{T}$ type (low voltage activated) calcium channels and/or Ih channels. These channels may serve to drive the cell towards action potential threshold following a hyperpolarizing pulse (inhibitory input), activating voltage gated sodium channels resulting in generation of action potentials. Thus, our model for the formation of offset responses in the SPON includes a convergent glycinergic input, a late arriving excitatory input onto the dendrites and a particular complement of membrane channels. Upon stimulation with a pure tone, the contralateral SPON receives a glycinergic that 
hyperpolarizes the neuron. Shortly thereafter, excitatory input from the $\mathrm{CN}$ arrives on the dendrites, but is unable to depolarize the cell enough to elicite an action potential. At the end of the stimulus, the hyperpolarizing input is removed and there is a large influx of cations through either T type (low voltage activated) calcium channels and/or Ih channels. This influx of cations activate voltage gated sodium channels and results in an action potential at the offset of a stimulus.

\section{Comparative issues}

An interesting feature of the SPON, much like the MSO (Grothe, 2000), is that it appears to exhibit considerable interspecies variation. It has been well established that the SPON projects to the IC (Coleman and Clerici, 1987; Saint Marie and Baker, 1990; Schofield, 1991). In fact, the rat SPON almost exclusively projects to the ipsilateral IC (Saldaña and Berrebi, 2000). The SPON, in some species (gerbil, guinea pig, cat), contain glycinergic and cholinergic neurons that project to the cochlear nucleus and cochlea (Warr, 1975; Strutz and Spatx, 1980; Robertson, 1985; Helfert et al., 1988; Winter et al., 1989). Thus, in some species this cell group has evolved to contain olivocochlear neurons that provide modulatory feedback to the cochlea and cochlear nucleus. The physiological response properties of SPON neurons appear to vary somewhat between species. Approximately half of gerbil SPON neurons are binaural and based on responses to sound stimuli, the gerbil SPON contains at least two distinct populations of neurons (Berhend et al., 2002, Dehmel et al., 2002). The majority of cat SPON/DMPO neurons are monaural (65\%) and about half respond only at the stimulus offset (53\%; Guinan et al., 1972). In contrast, the rat and rabbit SPON are largely monaural and nearly all of the neurons respond at the stimulus offset (Kuwada and Batra, 1999). Thus, the SPON may be a site within the SOC capable of evolutionary plasticity that is capable of modification to fit the specific needs of the animal. This variability of the SPON is not likely a result of increased sensitivity to high frequency sounds as the hearing range of rats (1 to $60 \mathrm{kHz})$, cats $(30 \mathrm{~Hz}$ to $50 \mathrm{kHz})$ and gerbils $(100 \mathrm{~Hz}$ to $60 \mathrm{kHz}$ ) overlap considerably. The differences in the anatomy and physiology of the SPON in these species may reflect specific needs related to environmental pressures (avoiding predators) or the particular patterns of species specific vocalizations. 


\section{Potential functional roles}

Neurons responding to the offset of pure tone stimuli have been recorded throughout the mammalian auditory pathway from the cochlear nucleus to the auditory cortex but likely make up a small fraction of auditory neurons (Guinan et al., 1972; Grinell, 1973; Aitkin and Prain, 1974; Grothe, 1994; Spitzer and Semple, 1995; Kuwada and Batra, 1997; He et al., 1997; He, 2001). Offset responses have been recorded in the auditory pathway of different species, ranging from mouse (Brand et al., 2000), rat (this study), rabbit (Kuwada and Batra, 1999), bat (Grothe, 1994) and cat (Guinan et al., 1972; He et al., 1997; He 2001). Thus, offset responses are widespread throughout the mammalian auditory system and likely carry information important to auditory processing. The offset activity that arises from the SPON is inhibitory, however offset information from other levels (IC, thalamus and cortex) may be excitatory. Nearly all (95\%) SPON neurons responded at the stimulus offset. Thus, detecting the stimulus offset is a distinguishing feature of the rat SPON and these neurons are very likely the major source of ascending offset inhibition. Although the role of offset responses in hearing is unclear, they appear to be necessary for duration tuning, gap detection and the precedence effect (discussed below). Offset responses recorded in the rat and rabbit SPON phase lock to amplitude modulated pure tones (Kuwada and Batra, 1999). The transient nature of the offset response and the sensitivity to temporal features of sound implicates a role in processing complex signals, such as vocalizations or other behaviorally relevant sounds.

In the description of basic response properties of SPON neurons (chapter 4) we found three different types of offset responses: offset-transient (responding to pure tones with only one spike per stimulus), offset-choppers (responding with 2 or more regularly timed spikes) and offset-sustained (responses that lasted more than $20 \mathrm{~ms}$ ). Offset-choppers and offset-sustained neurons, which combined made up $46 \%$ of the population, displayed some sensitivity for stimulus duration and intensity. Thus, these two categories of SPON neurons may be directly involved in duration sensitivity in the IC, which may also require intensity information. The role of offset transient neurons, which made up $41 \%$ of all SPON neurons and showed little sensitivity for intensity or stimulus duration, may be limited to simply indicating the end of the stimulus or fluctuations in amplitude. Even though offset transient neurons did not show duration selectivity, this does not exclude them from contributing to duration sensitivity in the 
IC.

The potential functional role of the SPON in auditory processing will be examined in the following section. The subsequent section will focus on gap detection and the potential role of the SPON in detecting gaps between stimuli. In addition, the potential role of the SPON in duration tuning, coding amplitude modulation and the precedence effect will be examined.

\section{Gap detection}

Most adult rat vocalizations consist of between two and twenty short bursts of constant frequency and variable duration ( $3 \mathrm{~ms}$ up to 2 seconds) separated by gaps of between 25 and 200 ms (Brudzynski et al., 1993; Brudzynski and Ociepa, 1992; Francis, 1977). Since rats use vocalizations with multiple short components for communication, it is crucial that the rat auditory system detects and responds to each component of the call. Having these short calls so temporally close, might necessitate an inhibitory input that arrives at the end of a sound to "turn off" an auditory neuron so that it can precisely detect the end of a sound and respond precisely to the onset of the next incoming signal. Offset inhibitory input might be especially important for neurons with sustained responses, especially when the evoked spike activity outlasts the stimulus. Detection of gaps or silent periods between stimuli is thought to play an important role in processing complex sounds and speech perception (Barsz et al., 1998).

The offset inhibition arising from the SPON may serve to detect gaps between closely placed sounds or components of sounds. Gap detection is often tested using a forward masking paradigm, where a leading stimulus is presented and a lagging stimulus is played with some variable delay. Detection of gaps between stimuli means that a neuron or the animal detects two stimuli even when the stimuli are only separated by a few milliseconds; a gap is detected between the stimuli. Thus, brief gaps in sound are detected in the nervous system by a cessation in neuronal activity that may result from inhibitory innervation.

Mammalian auditory nerve fibers have been shown to detect gaps between sounds as short as $2 \mathrm{~ms}$ (Zhang et al., 1990). Interestingly, gap thresholds for neurons in the IC are slightly higher than those described for the auditory nerve; minimal gap thresholds for IC neurons range between 2 and $4.2 \mathrm{~ms}$, and IC gap thresholds are very similar to behavioral gap thresholds 
(Walton et al., 1997). The longer minimal gap thresholds in IC neurons and in behavioral data provide evidence for a mechanism that lengthens gap thresholds. One potential mechanism that could be involved in lengthening gap thresholds would be an inhibitory input arriving at the end of a stimulus. This brings about an interesting question: If the auditory nerve can detect short gaps between sounds, why involve a mechanism to that lengthens gap thresholds? The reason for this may be to reduce some of the jitter present in detecting the end of a stimulus. Having a well-timed offset inhibition will allow a precise indication of the end of the stimulus and allow the neurons to respond to the next stimulus within a very short timed period. Accompanying an inhibitory input at the end of the stimulus comes a decrease in gap sensitivity of 1 to $2 \mathrm{~ms}$.

It is very likely that not all SPON neurons are involved in gap detection. Offset choppers and offset sustained neurons are unlikely candidates for gap detectors because of the length of the offset response in these neurons. The inhibition arising from offset choppers, consisting of two or more regularly timed spikes and often lasting more than ten milliseconds, would not be compatible with detection of a $2 \mathrm{~ms}$ gap in sound. For the same reason, the input from offset sustained neurons are not likely involved in gap detection. By definition, offset-sustained neurons have responses that last for longer than $20 \mathrm{~ms}$. The length of this inhibitory input makes offset sustained neurons poor candidates for involvement in detecting short gaps between sounds. However, SPON neurons with offset transient responses could be involved in gap detection because their response typically consists of a single spike to a BF tone. This welltimed and brief offset inhibition is well suited for a role in detecting gaps between stimuli. Thus, offset transient neuron may play a role in gap detection.

Presbycusis, or age related hearing loss, is described as a decline in auditory sensitivity that occurs with the normal progression of age. Moreover, presbycusis has been at least partially attributed to a decrease in GABA in the IC (Caspary et al., 1999). Minimum gap detection thresholds are elevated in older animals and this has been described using both in vivo recordings (Walton et al., 1998) and a behavioral paradigm (Ison et al., 1998). This taken with the fact that gap detection thresholds in older subjects can be lowered with application of drugs that elevate GABA levels (Gleich et al., 2002) provides evidence of an inhibitory input that shapes gap thresholds in the IC. Additionally, GABA has been shown to increase sensitivity to gap 
detection by lowering minimal gap thresholds in the IC (Gleich et al., 2002) and ventral cochlear nucleus (Backoff, 1997). Moreover, many neurons in the rat IC show a poststimulus suppression that can last up to $270 \mathrm{~ms}$ after the stimulus (Finlayson, 1999) and neurons in the bat IC also show a poststimulus suppression that is mediated by GABA (Bauer et al., 2000). This indicates that IC neurons can detect short gaps between stimuli, but the response to the second stimulus (if it occurs less that $270 \mathrm{~ms}$ after the first) is somewhat attenuated. These data, taken together indicate that there is a GABAergic input to the IC that is involved in gap detection. The SPON is a source of GABAergic inhibition to the IC and is therefore a candidate for a role in gap detection.

The minimal gap thresholds recorded from the auditory nerve provides evidence that inhibition is not required to accurately detect short gaps between stimuli. However, the literature does provide some convincing evidence that inhibition does play a role in shaping gap detection thresholds in the IC. A role for the SPON in gap detection could be examined using an in vivo or behavioral preparation. In an in vivo preparation, spike activity of IC neurons would be recorded. In a behavioral paradigm, animals would have to be trained to detect gaps between stimuli and respond appropriately. Since there are numerous sources of GABA to the IC, the SPON would have to pharmacologically inactivated or lesioned to study the effect of this cell group in gap detection. Ideally a reversible pharmacological inactivation would be utilized, which would allow data to be collected before and after inactivation of the SPON. The appropriate stimulus would be a pair of tones played with varying gaps or delays between them. Data relating to minimum gap detection thresholds would be collected before and after inactivation of the SPON. Our working hypothesis is that the offset inhibition arising from the SPON is involved in shaping minimum gap detection thresholds in IC neurons and removing the offset inhibition would significantly change gap detection thresholds, especially for those IC neurons with sustained responses to tones.

SPON neurons may function as gap detectors to encode gaps between closely placed sounds or components of sounds. Brief gaps in sound are detected in the nervous system by a cessation in neuronal activity that may result from inhibitory innervation. Detection of gaps or silent periods between stimuli is thought to play an important role in processing complex sounds and speech perception (Barsz et al., 1998). Neurons in the mouse IC can detect gaps between 
two stimuli as short as $2 \mathrm{~ms}$ (Walton et al., 1997). Many neurons in the rat IC show poststimulus suppression that can last up to $270 \mathrm{~ms}$ after the stimulus (Finlayson, 1999) and in the bat this suppression is mediated by GABA (Bauer et al., 2000). Additionally, GABA has been shown to increase sensitivity to gap detection by lowering minimal gap thresholds (Gleich et al., 2002), further implicating a role for GABA in gap detection. These data, taken together indicate that there is a GABAergic input to the IC that arrives at the end of a stimulus or outlasts it by tens of milliseconds and that this input is involved in gap detection. The SPON is a likely source for this offset or persistent inhibition and is therefore a candidate for a role in gap detection.

\section{Duration tuning}

Naturally occurring sounds are characterized by the frequencies they contain, intensity, location and duration. Duration tuning is an auditory phenomenon where neurons respond selectively to stimuli of particular durations. Most adult rat vocalizations consist of between two and twenty individual calls of constant frequency and variable duration ( $3 \mathrm{~ms}$ up to 2 seconds; Brudzynski et al., 1992; Brudzynski and Ociepa, 1992; Francis, 1977). The duration of each individual call is likely an important aspect of the vocalization since frequency is relatively constant and duration tuned neurons may be necessary for the animal to interpret each of the individual calls. Duration tuned neurons can be "short pass" (responding only to short stimuli and not longer ones) or "band pass" (responding to a range of durations but not durations that are longer or shorter). Duration selective neurons respond weakly or not at all to short stimuli and more robustly to longer stimuli but reach a plateau such that the increase in spikes is not linearly related to the duration of the stimulus. Tuning to specific durations has been implicated in encoding vocalizations and may be necessary for separating biologically important sounds from unimportant sounds. For example, neurons in the frog midbrain have best durations that correspond to durations of communication calls (Gooler and Feng, 1992). A similar correlation between best durations and vocalizations has been described for bat IC neurons (Pinheiro et al., 1991; Casseday et al., 1994; Fuzessery, 1994).

Duration sensitivity is thought to be formed in the IC through a complex integration of excitatory and inhibitory inputs. About half (45\%) of the SPON neurons we tested were sensitive to duration and were long duration selective. This is the first demonstration of duration 
sensitivity below the level of the IC and means that some neurons in the IC may inherit duration sensitivity from lower centers. Slightly more than half (55\%) of mouse IC neurons are duration sensitive and most of these neurons $(\sim 70 \%)$ are selective for longer durations (sounds greater than $\sim 50 \mathrm{~ms}$ in duration) and respond at the stimulus offset (Brand et al., 2000). This combined with our SPON data, indicates that long duration selectivity (sounds $50 \mathrm{~ms}$ or longer) may be the most important type of duration tuning in the rat. Interestingly, few rat vocalizations are less than $50 \mathrm{~ms}$ in duration (Brudzynski and Oceipa, 1992). Duration tuning in IC neurons has been shown, through intracellular in vivo recordings, to be formed by the integration of precisely timed excitatory and inhibitory inputs (Casseday et al., 1994). The most recent model for duration tuned neurons requires: 1. excitatory and inhibitory input linked to the onset of the stimulus, 2. an inhibitory input linked to the duration of the sound and 3. an excitatory event linked to the sound offset. The excitatory response linked to the stimulus offset can be a response to an excitatory input or a rebound from inhibition. Further, application of the GABA receptor antagonist bicuculline abolishes duration tuning in most IC neurons (Casseday et al., 2000). Offset inhibition arising from the SPON may directly relay duration sensitivity to IC neurons, or may provide inhibition linked to the stimulus duration or cause an excitatory event at the offset through a rebound mechanism. Thus, the SPON may be an important part of the circuitry used to detect stimulus duration. However, the precise role that offset inhibition plays in creating duration tuning in the IC remains to be tested and would require single unit recordings from the IC during inactivation of the SPON.

\section{Amplitude modulation}

Interestingly, only about half of IC neurons are sensitive to duration (Brand et al., 2000; Ehrlich et al., 2000) and nearly half of SPON neurons are duration sensitive. However, the SPON projects widely to the IC (Fuentes et al., 1999). Thus, it is likely that SPON neurons project to IC neurons that are not involved in duration coding and that a fraction (likely more than half) of SPON neurons are dedicated to something other than duration sensitivity. Many naturally occurring sounds are characterized by rapid fluctuations in amplitude. SPON neurons respond to rapid amplitude fluctuations in SAM pure tones. Thus, some SPON neurons may be

involved in coding the temporal structure of complex sounds, rather than duration. IC neurons are selective for particular communication calls and this selectivity is abolished with bicuculline 
(bat; Pollak et al., 2002). This suggests that there is an inhibitory input to the IC that is relaying temporal information about sounds, particularly changes in amplitude. Unfortunately, responses to SAM have not been explored in the rat DNLL or VNLL. The SPON, being a major source of GABAergic innervation to the IC and sensitive to fluctuations in sound envelopes, is a candidate for encoding complex features of sound.

\section{Precedence effect}

The precedence effect is a feature of the auditory system that suppresses echo perception and is believed to play a role in localization of sound in a reverberant environment (Hartman, 1983). When two stimuli arrive from different sources, priority is given to the leading sound and the lagging sound is suppressed, provided the lagging sounds reaches the animal within a short time window (1-20) ms after the leading sound. If sufficient time separates both sounds, neurons respond normally to both. The suppression of the lagging sound is proposed to be the result of a long lasting inhibitory input (Litovsky and Delgutte, 2002) and inhibition arising from the DNLL has been implicated in the precedence effect (Litovsky and Yin, 1998). In order to suppress a lagging stimulus, the inhibition must outlast the stimulus by some period or arrive at the offset. However, few DNLL neurons have sustained responses to tones and the few sustained responses last only as long as the stimulus (Bajo et al., 1998; Kelly et al., 1998). Most VNLL neurons have sustained responses but the sustained portion of the response only contains few spikes and does not outlast the stimulus (Batra and Fitzpatrick, 1999). Thus, the DNLL and VNLL are not likely sources of long lasting inhibition to IC neurons. Long lasting inhibition might also arise from a persistent suppression caused by an inhibitory input during the stimulus. More than likely though, offset inhibition is involved. Thus, if an inhibitory input is responsible for the precedence effect, it likely arises from the SPON.

\section{Testing the role of the SPON}

We now know a fair amount about the anatomy and physiology of the rat SPON. However, it is unclear how this cell group impacts IC neurons and how this offset inhibition is integrated with other inhibitory inputs. Involvement of the SPON in duration and envelope 
coding leads us to the hypothesis that the SPON encodes temporal features of sounds and is necessary to properly recognize vocalizations and other complex sounds important for socialization and survival. However, blocking GABAergic inhibition in the IC with bicuculline will remove inhibition arising from all sources of GABA, including the SPON, DNLL, VNLL, LSO and intrinsic inputs. The role of offset inhibition in the IC can be tested with recordings made from the inferior colliculus while inactivating the SPON with kynurenic acid (a nonspecific antagonist of glutamate receptors) or blockers of sodium or calcium channels (TTX or $\mathrm{NiCl}_{2}$, respectively), which would block action potentials in SPON neurons. However, one must be aware that a sodium channel blocker will also likely abolish action potentials in passing axons. This would require a somewhat cumbersome electrode arrangement: a multibarrel electrode in the SPON and a single recording electrode in the ipsilateral IC. Particular care would be needed to ensure that pharmacological agents used to inactivate the SPON do not also affect nearby nuclei, namely the MNTB and LSO. Of particular interest would be how offset inhibition affects duration selectivity and phase locking to SAM pure tones in IC neurons. We hypothesize that responses to SAM will be overall less well timed (resulting in lower vector strengths) and duration sensitivity will be abolished during SPON inactivation.

\section{SUGGESTIONS}

Although this work has contributed to our knowledge of the SPON and provided evidence for a potential functional role, some important questions remain. First, it remains unclear if inhibitory input alone can explain the responses observed to both pure tone and SAM stimuli. We have shown that the offset response requires glycinergic input, but is excitatory input also required? The literature provides evidence that postinhibitory rebound can be evoked in vivo with application of GABA (Plenz and Kital, 1999), suggesting that excitatory input may not be required for offset responses. Consequently, we hypothesize that offset responses in SPON neurons arise purely from inhibitory rebound. To begin to explore this possibility, we have recorded from two SPON neurons, using multibarrel electrodes, in the presence of kynurenic acid. In both neurons, offset activity was unchanged by kynurenic acid. Interestingly, one neuron had very weak onset activity that preceded the offset, presumably resulting from excitatory input. During application of kynurenic acid, these onset spikes were abolished, but the offset activity was unchanged. The few onset spikes did recover after kynurenic acid was 
removed. This data provides evidence that kynurenic acid was in fact reaching the neuron and that the offset response does not require activation of glutamate receptors. Of course, additional recordings from SPON neurons in the presence of kynurenic acid are needed to verify this finding. This question might more easily be addressed in a slice preparation that includes the SPON-MNTB connection. We hypothesize that shocks to the MNTB will elicit IPSPs and postinhibitory rebound spikes in SPON neurons. If this is the case, it provides further evidence supporting the hypothesis that offset activity requires only inhibitory input. However, the occurrence of postinhibitory rebound spikes in the SPON after MNTB shocks does not rule out a role for glutamaterigc input in generating offset spikes.

Secondly, it is unknown how SPON neurons respond to natural occurring sounds, such as vocalizations. Many rat vocalizations are essentially ultrasonic pure tones of varying duration while others are more complex and contain large frequency modulations (Brudzynski et al., 1999). We hypothesize that SPON neurons will indicate the end of the quasi-pure tone vocalizations. However, responses to more complex stimuli may be more irregular and unpredictable. Studying the neuronal responses of SPON units to vocalizations may further clarify our understanding of the functional role of this cell group.

Thirdly, the distribution of SPON axons in the IC remains unclear. Our stereology data indicate that the IC contains nearly 400,000 neurons and the SPON contains only 2,400. Is the projection from the SPON spread evenly over the 400,000 neurons in the IC? Do SPON axons target all IC neurons within a given frequency lamina or is this input selective? For example, does the SPON target a fraction of IC neurons, possibly only excitatory or duration tuned neurons? Additionally, to what extent does the SPON target any of the lemniscal nuclei? The question regarding projection targets could be addressed with small injections of the anterograde tracer Phaseolus vulgaris-leucoagglutinin into the SPON, which will label SPON axons and terminals. The axons then could be followed into the IC and nuclei of the lateral lemniscus.

\section{CONCLUSION}

We have described the rat SPON as a prominent group of inhibitory neurons in the auditory brainstem that relays information about the stimulus offset and amplitude fluctuations in 
complex sounds from the contralateral ear to the IC. This work has provided us with enough data about the SPON to propose a functional role in auditory processing. Our data suggest that the rat SPON, through its inhibitory projection to IC neurons, is involved in processing temporal cues. These cues may be involved in duration selectivity and coding of sound envelopes, information necessary for understanding communication calls and other biologically relevant sounds. It is our hope that this work will contribute to our understanding of the auditory system and the neural mechanisms used to encode sound. 


\section{REFERENCES}

Abercrombie, M. 1946. Estimation of nuclear populations from microtome sections. Anatomical Record 94, 239-247.

Adams, J.C. 1979. Ascending projections to the inferior colliculus. J Comp Neurol 183, 519-38.

Adams, J.C. 1983. Cytology of periolivary cells and the organization of their projections in the cat. J Comp Neurol 215, 275-89.

Adams, J.C. 1997. Projections from octopus cells of the posterventral cochlear nucleus to the ventral nucleus of the lateral lemniscus in cat and human. Auditory Neuroscience 3, 335-350.

Adams, J.C., Mugnaini, E. 1984. Dorsal nucleus of the lateral lemniscus: a nucleus of GABAergic projection neurons. Brain Res Bull 13, 585-90.

Agar, E., Korkmaz, A., Bosnak, M., Demir, S., Ayyildiz, M., Marangoz, C. 1999. Do cochlear nuclei contribute to auditory lateralization? A stereological evaluation of neuron numbers. Ann Otol Rhinol Laryngol 108, 661-5.

Aitkin, L.M., Prain, S.M. 1974. Medial geniculate body: unit responses in the awake cat. J Neurophysiol 37, 512-21.

Aizenman, C.D., Linden, D.J. 1999. Regulation of the rebound depolarization and spontaneous firing patterns of deep nuclear neurons in slices of rat cerebellum. J Neurophysiol 82, 1697-709.

Aoki, E., Semba, R., Keino, H., Kato, K., Kashiwamata, S. 1988. Glycine-like immunoreactivity in the rat auditory pathway. Brain Res 442, 63-71.

Aschoff, A., Ostwald, J. 1987. Different origins of cochlear efferents in some bat species, rats, and guinea pigs. J Comp Neurol 264, 56-72.

Backoff, P.M., Shadduck Palombi, P., Caspary, D.M. 1997 Glycinergic and GABAergic inputs affect short-term suppression in the cochlear nucleus. Hear. Res. 110, 155-163

Bajo, V.M., Merchan, M.A., Lopez, D.E., Rouiller, E.M. 1993. Neuronal morphology and efferent projections of the dorsal nucleus of the lateral lemniscus in the rat. J Comp Neurol 334, $241-62$.

Bajo, V.M., Villa, A.E., de Ribaupierre, F., Rouiller, E.M. 1998. Discharge properties of single neurons in the dorsal nucleus of the lateral lemniscus of the rat. Brain Res Bull 47, 595-610.

Bando, T., Zambelli, A., Spencer, W.A. 1980. Rebound excitation and the rhythmic activity of the ventrobasal complex of the thalamus. Brain Res 192, 29-38.

Banks, M.I., Smith, P.H. 1992. Intracellular recordings from neurobiotin-labeled cells in brain 
slices of the rat medial nucleus of the trapezoid body. J Neurosci 12, 2819-37.

Barsz, K., Benson, P.K., Walton, J.P. 1998. Gap encoding by inferior collicular neurons is altered by minimal changes in signal envelope. Hear Res 115, 13-26.

Batra, R., Fitzpatrick, D.C. 1999. Discharge patterns of neurons in the ventral nucleus of the lateral lemniscus of the unanesthetized rabbit. J Neurophysiol 82, 1097-113.

Bauer, E.E., Klug, A., Pollak, G.D. 2000. Features of contralaterally evoked inhibition in the inferior colliculus. Hear Res 141, 80-96.

Baxter, C.F. 1970. The nature of GABA. In: Lajtha, A., (Ed.), Handbook of Neurochemistry, Vol. 3. Plenum Press, New York. pp. 289-353.

Bayon, A., Possani, L.D., Tapia, M., Tapia, R. 1977. Kinetics of brain glutamate decarboxylase. Interactions with glutamate, pyridoxal 5'-phosphate and glutamate-pyridoxal 5'-phosphate Schiff base. J Neurochem 29, 519-25.

Behrend, O., Brand, A., Kapfer, C., Grothe, B. 2002. Auditory response properties in the superior paraolivary nucleus of the gerbil. J Neurophysiol 87, 2915-28.

Benson, C.G., Potashner, S.J. 1990. Retrograde transport of [3H]glycine from the cochlear nucleus to the superior olive in the guinea pig. J Comp Neurol 296, 415-26.

Berrebi, A.S., Mugnaini, E. 1991. Distribution and targets of the cartwheel cell axon in the dorsal cochlear nucleus of the guinea pig. Anatomy and Embryology 183, 427-454.

Berrebi, A.S., Malmierca, M.S., Saldaña, E. 1997. Features of organization of the superior paraolivary nucleus of the rat, guinea pig and chinchilla, Association for Research in Otolaryngology, Vol. 20:570.

Beyerl, B.D. 1978. Afferent projections to the central nucleus of the inferior colliculus in the rat. Brain Res 145, 209-23.

Bledsoe, S.C., Jr., Snead, C.R., Helfert, R.H., Prasad, V., Wenthold, R.J., Altschuler, R.A. 1990. Immunocytochemical and lesion studies support the hypothesis that the projection from the medial nucleus of the trapezoid body to the lateral superior olive is glycinergic. Brain Res 517, 189-94.

Boudreau, J.C., Tsuchitani, C. 1968. Binaural interaction in the cat superior olive S segment. J Neurophysiol 31, 442-54.

Brand, A., Urban, R., Grothe, B. 2000. Duration tuning in the mouse auditory midbrain. J Neurophysiol 84, 1790-9.

Brawer, J.R., Morest, D.K., Kane, E.C. 1974. The neuronal architecture of the cochlear nucleus 
of the cat. J Comp Neurol 155, 251-300.

Bredt, D.S., Snyder, S.H. 1992. Nitric oxide, a novel neuronal messenger. Neuron 8, 3-11.

Brown, J.C., Howlett, B. 1972. The olivo-cochlear tract in the rat and its bearing on the homologies of some constituent cell groups of the mammalian superior olivary complex: a thiocholine study. Acta Anat 83, 505-26.

Browner, R.H., Webster, D.B. 1975. Projections of the trapezoid body and the superior olivary complex of the Kangaroo rat (Dipodomys merriami). Brain Behav Evol 11, 322-54.

Brudzynski, S.M., Ociepa, D. 1992. Ultrasonic vocalization of laboratory rats in response to handling and touch. Physiol Behav 52, 655-60.

Brudzynski, S.M., Kehoe, P., Callahan, M. 1999. Sonographic structure of isolation-induced ultrasonic calls of rat pups. Dev Psychobiol 34, 195-204.

Brudzynski, S.M., Bihari, F., Ociepa, D., Fu, X.W. 1993. Analysis of $22 \mathrm{kHz}$ ultrasonic vocalization in laboratory rats: long and short calls. Physiol Behav 54, 215-21.

Brugge, J.F. 1991. An Overview of Central Auditory Processing. In: Richard R. Fay, Popper, A.N., (Eds.), The Mammalian Auditory Pathway: Neurophysiology, Vol. 2. Springer-Verlag New York, Inc., New York, NY. pp. 1-34.

Burger, R.M., Pollak, G.D. 2001. Reversible inactivation of the dorsal nucleus of the lateral lemniscus reveals its role in the processing of multiple sound sources in the inferior colliculus of bats. J Neurosci 21, 4830-43.

Caicedo, A., Herbert, H. 1993. Topography of descending projections from the inferior colliculus to auditory brainstem nuclei in the rat. J. Comp. Neurol. 328, 377-392.

Caicedo, A., Eybalin, M. 1999. Glutamate receptor phenotypes in the auditory brainstem and mid-brain of the developing rat. Eur J Neurosci 11, 51-74.

Campistron, G., Buijs, R.M., Geffard, M. 1986. Glycine neurons in the brain and spinal cord. Antibody production and immunocytochemical localization. Brain Res 376, 400-5.

Cant, N.B. 1992. The cochlear nucleus: neuronal types and their synaptic organization. In: Webster, W.R., Popper, A.N., Fay, R.R., (Eds.), Mammalian Auditory pathways: Neuroanatomy. Springer-Verlag, New York, NY.

Cant, N.B., Benson, C.G., Schofield, B.R. 1999. Projections from the superior paraolivary nucleus to the inferior colliculus in guinea pigs and gerbil, Association for Research in Otolaryngology, Vol. 22:883.

Casey, M.A. 1990. The effects of aging on neuron number in the rat superior olivary complex. 
Neurobiol Aging 11, 391-4.

Caspary, D.M., Backoff, P.M., Finlayson, P.G., Palombi, P.S. 1994. Inhibitory inputs modulate discharge rate within frequency receptive fields of anteroventral cochlear nucleus neurons. J Neurophysiol 72, 2124-33.

Caspary, D.M., Holder, T.M., Hughes, L.F., Milbrandt, J.C., McKernan, R.M., Naritoku, D.K. 1999. Age-related changes in $\operatorname{GABA}(\mathrm{A})$ receptor subunit composition and function in rat auditory system. Neuroscience $93,307-12$.

Casseday, J.H., Ehrlich, D., Covey, E. 1994. Neural tuning for sound duration: role of inhibitory mechanisms in the inferior colliculus. Science 264, 847-50.

Casseday, J.H., Ehrlich, D., Covey, E. 2000. Neural measurement of sound duration: control by excitatory-inhibitory interactions in the inferior colliculus. J Neurophysiol 84, 1475-87.

Chang, Y.C., Gottlieb, D.I. 1988. Characterization of the proteins purified with monoclonal antibodies to glutamic acid decarboxylase. J Neurosci 8, 2123-30.

Coleman, J.R., Clerici, W.J. 1987. Sources of projections to subdivisions of the inferior colliculus in the rat. J Comp Neurol 262, 215-26.

Colombo, A., Saldaña, E., Berrebi, A.S. 1996. Efferent projections of the rat superior paraolivary nucleus, Association for Research in Otolaryngology, Vol. 19:483.

Cooper, A.J., Stanford, I.M. 2000. Electrophysiological and morphological characteristics of three subtypes of rat globus pallidus neurone in vitro. J Physiol 527 Pt 2, 291-304.

Covey, E., Jones, D.R., Casseday, J.H. 1984. Projections from the superior olivary complex to the cochlear nucleus in the tree shrew. J Comp Neurol 226, 289-305.

Curtis, D.R., Duggan, A.W., Johnston, G.A. 1971a. The specificity of strychnine as a glycine antagonist in the mammalian spinal cord. Exp Brain Res 12, 547-65.

Curtis, D.R., Duggan, A.W., Felix, D., Johnston, G.A. 1971b. Bicuculline, an antagonist of GABA and synaptic inhibition in the spinal cord of the cat. Brain Res 32, 69-96.

Dehmel, S., Doerrscheidt, G., Reubsamen, R. 2002. Electrophysiological characterization of neurons in the superior paraolivary nucleus of the gerbil (Meriones unguiculatus). Hearing Research in press.

Denner, L.A., Wu, J.Y. 1985. Two forms of rat brain glutamic acid decarboxylase differ in their dependence on free pyridoxal phosphate. J Neurochem 44, 957-65.

Dirkx, R., Jr., Thomas, A., Li, L., Lernmark, A., Sherwin, R.S., De Camilli, P., Solimena, M. 1995. Targeting of the $67-\mathrm{kDa}$ isoform of glutamic acid decarboxylase to intracellular organelles 
is mediated by its interaction with the NH2- terminal region of the $65-\mathrm{kDa}$ isoform of glutamic acid decarboxylase. J Biol Chem 270, 2241-6.

Doucet, J.R., Ryugo, D.K. 2002. Projections of multipolar neurons in the ventral cochlear nucleus to the lateral superior olive, Association for Research in Otolaryngology, Vol. 25:30.

Edwards, S.B., Ginsburgh, C.L., Henkel, C.K., Stein, B.E. 1979. Sources of subcortical projections to the superior colliculus in the cat. J Comp Neurol 184, 309-29.

Ehrlich, D., Casseday, J.H., Covey, E. 1997. Neural tuning to sound duration in the inferior colliculus of the big brown bat, Eptesicus fuscus. J Neurophysiol 77, 2360-72.

Erlander, M.G., Tobin, A.J. 1991. The structural and functional heterogeneity of glutamic acid decarboxylase: a review. Neurochemical Research 16, 215-226.

Esclapez, M., Houser, C.R. 1999. Up-regulation of GAD65 and GAD67 in remaining hippocampal GABA neurons in a model of temporal lobe epilepsy. J Comp Neurol 412, 488505 .

Esclapez, M., Tillakaratne, N.J., Kaufman, D.L., Tobin, A.J., Houser, C.R. 1994. Comparative localization of two forms of glutamic acid decarboxylase. J Neurosci 14, 1834-55.

Faingold, C.L., Travis, M.A., Gehlbach, G., Hoffmann, W.E., Jobe, P.C., Laird, H.E., 2nd, Caspary, D.M. 1986. Neuronal response abnormalities in the inferior colliculus of the genetically epilepsy-prone rat. Electroencephalogr Clin Neurophysiol 63, 296-305.

Faye-Lund, H. 1986. Projection from the inferior colliculus to the superior olivary complex in the albino rat. Anat. Embryol. 175, 35-52.

Feng, A.S., Hall, J.C., Gooler, D.M. 1990. Neural basis of sound pattern recognition in anurans. Prog Neurobiol 34, 313-29.

Fessenden, J.D., Altschuler, R.A., Seasholtz, A.F., Schacht, J. 1999. Nitric oxide/cyclic guanosine monophosphate pathway in the peripheral and central auditory system of the rat. $\mathbf{J}$ Comp Neurol 404, 52-63.

Finlayson, P.G. 1999. Post-stimulatory suppression, facilitation and tuning for delays shape responses of inferior colliculus neurons to sequential pure tones. Hear Res 131, 177-94.

Finlayson, P.G., Caspary, D.M. 1989. Synaptic potentials of chinchilla lateral superior olivary neurons. Hear Res 38, 221-8.

Finlayson, P.G., Adam, T.J. 1997. Excitatory and inhibitory response adaptation in the superior olive complex affects binaural acoustic processing. Hear Res 103, 1-18.

Francis, R.L. 1977. 22-kHz calls by isolated rats. Nature 265, 236-8. 
Friauf, E. 1995. C-fos immunocytochemical evidence for acoustic pathway mapping in rats. Behav Brain Res 66, 217-24.

Friauf, E., Ostwald, J. 1988. Divergent projections of physiologically characterized rat ventral cochlear nucleus neurons as shown by intra-axonal injection of horseradish peroxidase. Exp. Brain Res. 73, 263-284.

Friauf, E., Fritschy, J.M., Kuchenbrod, T. 1998. Differential distribution of GABA-A receptor subunits in the auditory brainstem of adult rats, Association for Research in Otolaryngology, Vol. 21:457.

Friauf, F., Hammerschmidt, B., Kirsch, J. 1997. Development of adult type inhibitory glycine receptors in the central auditory system of rats. The Journal of Comparative Neurology 385, 117134.

Frisina, R.D., Smith, R.L., Chamberlain, S.C. 1990. Encoding of amplitude modulation in the gerbil cochlear nucleus: I. A hierarchy of enhancement. Hear Res 44, 99-122.

Fuentes, V., Berrebi, A.S., Saldaña, E. 1999. Trajectory, morphology and distribution of axons of the superior paraolivary nucleus that innervate the inferior colliculus in the rat, Association for Research in Otolaryngology, Vol. 22:885.

Fuzessery, Z.M., Hall, J.C. 1996. Role of GABA in shaping frequency tuning and creating FM sweep selectivity in the inferior colliculus. J Neurophysiol 76, 1059-73.

Gates, T.S., Weedman, D.L., Pongstaporn, T., Ryugo, D.K. 1996. Immunocytochemical localization of glycine in a subset of cartwheel cells of the dorsal cochlear nucleus in rats. Hear Res 96, 157-66.

Gleich, O., Hamann, I., Klump, G.M., Kittel, M., Strutz, J. 2002. Preliminary evidence for pharamcological improveement of impaired gap detection in gerbils, Association for Research in Otolaryngology, Vol. 25:954.

Glendenning, K.K., Hutson, K.A., Nudo, R.J., Masterton, R.B. 1985. Acoustic chiasm II: Anatomical basis of binaurality in lateral superior olive of cat. J Comp Neurol 232, 261-85.

Godfrey, D.A., Kiang, N.Y., Norris, B.E. 1975. Single unit activity in the posteroventral cochlear nucleus of the cat. J Comp Neurol 162, 247-68.

Godfrey, D.A., Farms, W.B., Godfrey, T.G., Mikesell, N.L., Liu, J. 2000. Amino acid concentrations in rat cochlear nucleus and superior olive. Hear Res 150, 189-205.

Goldberg, J.M., Brown, P.B. 1969. Response of binaural neurons of dog superior olivary complex to dichotic tonal stimuli: some physiological mechanisms of sound localization. J Neurophysiol 32, 613-36. 
Gonzalez-Hernandez, T., Mantolan-Sarmiento, B., Gonzalez-Gonzalez, B., Perez-Gonzalez, H. 1996. Sources of GABAergic input to the inferior colliculus of the rat. J Comp Neurol 372, 30926.

Gooler, D.M., Feng, A.S. 1992. Temporal coding in the frog auditory midbrain: the influence of duration and rise-fall time on the processing of complex amplitude-modulated stimuli. J Neurophysiol 67, 1-22.

Grenier, F., Timofeev, I., Steriade, M. 1998. Leading role of thalamic over cortical neurons during postinhibitory rebound excitation. Proc Natl Acad Sci U S A 95, 13929-34.

Grinnell, A.D. 1973. Rebound excitation (off-responses) following nonneural suppression in the cochlear nucleusof echolocating bats. Journal of Comparative Physiology 82, 179-194.

Grothe, B. 1994. Interaction of excitation and inhibition in processing of pure tone and amplitude-modulated stimuli in the medial superior olive of the mustached bat. J Neurophysiol 71, 706-21.

Grothe, B., Neuweiler, G. 2000. The function of the medial superior olive in small mammals: temporal receptive fields in auditory analysis. J Comp Physiol [A] 186, 413-23.

Grothe, B., Vater, M., Casseday, J.H., Covey, E. 1992. Monaural interaction of excitation and inhibition in the medial superior olive of the mustached bat: an adaptation for biosonar. Proc Natl Acad Sci U S A 89, 5108-12.

Guinan, J. 1968. Firing patterns and location of single auditory units in the brainstem (superior olivary complex) of anesthetized cats. Sc. D., Mass. Inst. Technol.

Guinan, J.J., Guinan, S.S., Norris, B.E. 1972a. Single auditory units in the superior olivary complex I:responses to sounds and classifiation based on physiological properties. International Journal of Neuroscience 4, 101-120.

Guinan, J.J., Norris, B.E., Guinan, S.S. 1972b. Single auditory units in the superior olivary complex II: Locations of unit categories and tonotopic organization. Int. J. Neurosci. 4, 147-166.

Gundersen, H.J. 1986. Stereology of arbitrary particles. A review of unbiased number and size estimators and the presentation of some new ones, in memory of William R. Thompson. J Microsc 143, 3-45.

Gundersen, H.J. 1988. The nucleator. J Microsc 151, 3-21.

Gundersen, H.J., Jensen, E.B. 1987. The efficiency of systematic sampling in stereology and its prediction. J Microsc 147 ( Pt 3), 229-63.

Gundersen, H.J., Bagger, P., Bendtsen, T.F., Evans, S.M., Korbo, L., Marcussen, N., Moller, A., 
Nielsen, K., Nyengaard, J.R., Pakkenberg, B., et al. 1988. The new stereological tools: disector, fractionator, nucleator and point sampled intercepts and their use in pathological research and diagnosis. APMIS 96, 857-81.

Harrison, J.M., Warr, W.B. 1962. A study of the cochlear nuclei and ascending auditory pathways of the medulla. Journal of Comparative Neurology 119, 341-380.

Harrison, J.M., Irving, R. 1966. Ascending connections of the anterior ventral cochlear nucleus in the rat. J Comp Neurol 126, 51-63.

Hartmann, W.M. 1983. Localization of sound in rooms. J Acoust Soc Am 74, 1380-91.

He, J. 2001. On and off pathways segregated at the auditory thalamus of the guinea pig. J Neurosci 21, 8672-9.

He, J., Hashikawa, T., Ojima, H., Kinouchi, Y. 1997. Temporal integration and duration tuning in the dorsal zone of cat auditory cortex. J Neurosci 17, 2615-25.

Helfert, R.H., Aschoff, A. 1997. Superior olivary complex and nuclei of the lateral lemniscus. In: Ehret, G., Romand, R., (Eds.), The central auditory system. Oxford University Press, New York.

Helfert, R.H., Bonneau, J.M., Wenthold, R.J., Altschuler, R.A. 1989. GABA and glycine immunoreactivity in the guinea pig superior olivary complex. Brain Res. 6, 269-286.

Henkel, C.K., Spangler, K.M. 1983. Organization of the efferent projections of the medial superior olivary nucleus in the cat as revealed by HRP and autoradiographic tracing methods. J Comp Neurol 221, 416-28.

Holt, G.A., Berrebi, A.S. 1999. Ultrastructure of the superior paraolivary nucleus (SPON) of the rat, Association for Research on Otolaryngology, Vol. 20:277.

Horvath, M., Kraus, K.S., Illing, R.B. 2000. Olivocochlear neurons sending axon collaterals into the ventral cochlear nucleus of the rat. J Comp Neurol 422, 95-105.

Howard, C.V., Reed, M.G. 1998. Unbiased Stereology: Three-Dimensional Measurements in Microscopy Springer-Verlag New York, Inc., New York.

Hubel, D.H., Wiesle, T.N. 1961. Integrative action in the cat's lateral geniculate body. Journal of Physiology 155, 385-398.

Idrizbegovic, E., Canlon, B., Bross, L.S., Willott, J.F., Bogdanovic, N. 2001. The total number of neurons and calcium binding protein positive neurons during aging in the cochlear nucleus of CBA/CaJ mice: a quantitative study. Hear Res 158, 102-15.

Ison, J.R., Agrawai, P., Pak, J., Vaughn, W.J., 1998. Changes in temporal acuity with age and with hearing impairment in the mouse: A study of the acoustic startle reflex and its inhibition by brief decrements in noise level. J. Acoust. Soc. Am. 104, 1696-1704. 
Irving, R., Harrison, J.M. 1967. The superior olivary complex and audition: a comparative study. J Comp Neurol 130, 77-86.

Kaufman, D.L., Houser, C.R., Tobin, A.J. 1991. Two forms of the gamma-aminobutyric acid synthetic enzyme glutamate decarboxylase have distinct intraneuronal distributions and cofactor interactions. J Neurochem 56, 720-3.

Kelly, J.B., Masterton, B. 1977. Auditory sensitivity of the albino rat. J Comp Physiol Psychol 91, 930-6.

Kelly, J.B., Buckthought, A.D., Kidd, S.A. 1998a. Monaural and binaural response properties of single neurons in the rat's dorsal nucleus of the lateral lemniscus. Hear Res 122, 25-40.

Kelly, J.B., Liscum, A., van Adel, B., Ito, M. 1998b. Projections from the superior olive and lateral lemniscus to tonotopic regions of the rat's inferior colliculus. Hear Res 116, 43-54.

Kerr, D.I., Ong, J., Johnston, G.A., Abbenante, J., Prager, R.H. 1989. Antagonism at GABAB receptors by saclofen and related sulphonic analogues of baclofen and GABA. Neurosci Lett $107,239-44$.

King, A.J., Jiang, Z.D., Moore, D.R. 1998. Auditory brainstem projections to the ferret superior colliculus: anatomical contribution to the neural coding of sound azimuth. J Comp Neurol 390, $342-65$.

Kingsley, R.E. 1996. Chapter 10 The Auditory System. In: Satterfield, T.S., (Ed.), Concise Text of Neuroscience. Williams and Wilkins, Baltimore, MD. pp. 345-381.

Klinke, G.J. 2000. The Laboratory Rat. The Handbook of Experimental Animal Series Academic Press, San Diego, CA.

Klug, A., Park, T.J., Pollak, G.D. 1995. Glycine and GABA influence binaural processing in the inferior colliculus of the mustache bat. J Neurophysiol 74, 1701-13.

Koch, U., Grothe, B. 1998. GABAergic and glycinergic inhibition sharpens tuning for frequency modulations in the inferior colliculus of the big brown bat. Journal of Neurophysiology 80, 7182.

Kolston, J., Osen, K.K., Hackney, C.M., Ottersen, O.P., Storm-Mathisen, J. 1992. An atlas of glycine- and GABA-like immunoreactivity and colocalization in the cochlear nuclear complex of the guinea pig. Anat. Embryol. 186, 443-465.

Kulesza, R.J., Berrebi, A.S. 2000. The superior paraolivary nucleus of the rat is a GABAergic nucleus. Journal of the Association for Research in Otolaryngology 1, 255-269.

Kulesza, R.J., Holt, G.A., Spirou, G.A., Berrebi, A.S. 2000. Intracellular labeling of axonal collaterals of SPON neurons, Association for Reseearch in Otolaryngology, Vol. 23:132. 
Kulesza, R.J., Viñeula, A., Saldana, E., Berrebi, A.S. 2002. Unbiased stereological estimates of neuron number in subcortical auditory nuclei of the rat. Hear Res 168 (1-2) 12-24.

Kumoi, K., Saito, N., Tanaka, C. 1993. Immunohistochemical localization of gammaaminobutyric acid- and aspartate-containing neurons in the guinea pig superior olivary complex. Hear Res 68, 173-9.

Kuwabara, N., Zook, J.M. 1991. Classification of the principal cells of the medial nucleus of the trapezoid body. J Comp Neurol 314, 707-20.

Kuwabara, N., Zook, J.M. 1992a. Inputs to the superior paraolivary nucleus, Society for Neuroscience, Vol. 18:1193.

Kuwabara, N., Zook, J.M. 1992b. Projections to the medial superior olive from the medial and lateral nuclei of the trapezoid body in rodents and bats. J Comp Neurol 324, 522-38.

Kuwabara, N., Zook, J.M. 1993. Local collaterals from the medial superior olive in the gerbil., ARO midwinter Meeting, Vol. 16:499.

Kuwabara, N., Zook, J.M. 1999. Local collateral projections from the medial superior olive to the superior paraolivary nucleus in the gerbil. Brain Res 846, 59-71.

Kuwabara, N., DiCaprio, R.A., Zook, J.M. 1991. Afferents to the medial nucleus of the trapezoid body and their collateral projections. J. Comp. Neurol. 314, 684-706.

Kuwada, S., Batra, R. 1999. Coding of sound envelopes by inhibitory rebound in neurons of the superior olivary complex in the unanesthetized rabbit. J Neurosci 19, 2273-87.

Labelle, R.E. 1997. The connectivity and ultrastructure of the dorsal nucleus of the lateral lemniscus of the albino rat. Master's Thesis, Carleton University, Ottawa, Canada.

LeBeau, F.E., Rees, A., Malmierca, M.S. 1996. Contribution of GABA- and glycine-mediated inhibition to the monaural temporal response properties of neurons in the inferior colliculus. $\mathbf{J}$ Neurophysiol 75, 902-19.

Ledoux, J.E., Ruggiero, D.A., Forest, R., Stornetta, R., Reis, D.J. 1987. Topographic organization of convergent projections to the thalamus from the inferior colliculus and spinal cord in the rat. J Comp Neurol 264, 123-46.

Legay, F., Henry, S., Tappaz, M. 1987. Evidence for two distinct forms of native glutamic acid decarboxylase in rat brain soluble extract: an immunoblotting study. J Neurochem 48, 1022-6.

Li, L., Wu, S.H., Zhang, D.X., Kelly, J.B. 1995. GABAergic projections to the central nucleus of the inferior colliculus of the rat: a combination of fluorogold retrograde tracing with immunocytochemistry, Society for Neuroscience Abstracts, Vol. 25:. 
Litovsky, R.Y., Yin, T.C. 1998. Physiological studies of the precedence effect in the inferior colliculus of the cat. II. Neural mechanisms. J Neurophysiol 80, 1302-16.

Litovsky, R.Y., Delgutte, B. 2002. Neural correlates of the precedence effect in the inferior colliculus: effect of localization cues. J Neurophysiol 87, 976-94.

Lorente de No, R. 1981. The primary acoustic nuclei Raven, New York.

Lu, Y., Jen, P.H. 2001. GABAergic and glycinergic neural inhibition in excitatory frequency tuning of bat inferior collicular neurons. Exp Brain Res 141, 331-9.

Manzoni, O., Bockaert, J. 1993. Nitric oxide synthase activity endogenously modulates NMDA receptors. J Neurochem 61, 368-70.

Martin, D.L., Rimvall, K. 1993. Regulation of gamma-aminobutyric acid synthesis in the brain. Journal of Neurochemistry 60, 395-407.

Martin, D.L., Martin, S.B., Wu, S.J., Espina, N. 1991. Regulatory properties of brain glutamate decarboxylase (GAD): the apoenzyme of GAD is present principally as the smaller of two molecular forms of GAD in brain. J Neurosci 11, 2725-31.

McAlpine, D., Palmer, A.R. 2002. Blocking GABAergic inhibition increases sensitivity to sound motion cues in the inferior colliculus. J Neurosci 22, 1443-53.

McCormick, D.A., Pape, H.C. 1990. Properties of a hyperpolarization-activated cation current and its role in rhythmic oscillation in thalamic relay neurones. J Physiol 431, 291-318.

Merchan, M.A., Berbel, P. 1996. Anatomy of the ventral nucleus of the lateral lemniscus in rats: a nucleus with a concentric laminar organization. J Comp Neurol 372, 245-63.

Merchan, M.A., Saldana, E., Plaza, I. 1994. Dorsal nucleus of the lateral lemniscus in the rat: concentric organization and tonotopic projection to the inferior colliculus. J Comp Neurol 342, 259-78.

Moore, D.R. 1988. Auditory brainstem of the ferret: sources of projections to the inferior colliculus. J Comp Neurol 269, 342-54.

Moore, J.K., Moore, R.Y. 1987. Glutamic acid decarboxylase-like immunoreactivity in brainstem auditory nuclei of the rat. J. Comp. Neurol. 260, 157-174.

Moore, M.J., Caspary, D.M. 1983. Strychnine blocks binaural inhibition in lateral superior olivary neurons. J Neurosci 3, 237-42.

Moore, R.Y., Goldberg, J.M. 1966. Projections of the inferior colliculus in the monkey. Exp Neurol 14, 429-38. 
Morest, D.K. 1968. The growth of synaptic endings in the mammalian brain: a study of the calyces of the trapezoid body. Z Anat Entwicklungsgesch 127, 201-20.

Moriizumi, T., Hattori, T. 1992. Anatomical and functional compartmentalization of the subparafascicular thalamic nucleus in the rat. Exp Brain Res 90, 175-9.

Mugnaini, E., Dahl, A.L. 1983. Zinc-aldehyde fixation for light-microscopic immunocytochemistry of nervous tissues. J Histochem Cytochem 31, 1435-8.

Mugnaini, E., Oertel, W.H. 1985. An atlas of the distribution of GABAergic neurons and terminals in the rat CNS as revealed by GAD immunohistochemistry. In: Björklund, A., Hökfelt, T., (Eds.), Handbook of Chemical Neuroanatomy. Vol. 4: GABA and Neuropeptides in the CNS, Part I., Vol. 4. Elsevier Science Publishers, Amsterdam. pp. 436-608.

Mugnaini, E., Warr, W.B., Osen, K.K. 1980. Distribution and light microscopic features of granule cells in the cochlear nuclei of cat, rat, and mouse. J Comp Neurol 191, 581-606.

Nicholls, D. 1994. Amino acids as transmitters, Proteins, Transmitters and Synapses. Blackwell Science.

Nordeen, K.W., Killackey, H.P., Kitzes, L.M. 1983. Ascending auditory projections to the inferior colliculus in the adult gerbil, Meriones unguiculatus. J Comp Neurol 214, 131-43.

O'Neill, W.E., Suga, N. 1982. Encoding of target range and its representation in the auditory cortex of the mustached bat. J Neurosci 2, 17-31.

Oertel, D. 1983. Synaptic responses and electrical properties of cells in brain slices of the mouse anteroventral cochlear nucleus. J Neurosci 3, 2043-53.

Oertel, W., Schmechel, D., Mugnaini, E., Tappaz, M., Kopin, I. 1981. Immunocytochemical localization of glutamate decarboxylase in rat cerebellum with a new antiserum. Neuroscience 6, 2715-2735.

Okoyama, S., Moriizumi, T., Kitao, Y., Kawano, J., Kudo, M. 1995. Anatomical plasticity in the medial superior olive following ablation of the inferior colliculus in neonatal and adult rats. Hear Res 88, 71-8.

Oliver, D.L., Kuwada, S., Yin, T.C., Haberly, L.B., Henkel, C.K. 1991. Dendritic and axonal morphology of HRP-injected neurons in the inferior colliculus of the cat. J Comp Neurol 303, 75-100.

Ollo, C., Schwartz, I.R. 1979. The superior olivary complex in C57BL/6 mice. Am J Anat 155, 349-73.

Ordronneau, P., Lindstrom, P.B., Petrusz, P. 1981. Four unlabeled antibody bridge techniques: a 
comparison. J Histochem Cytochem 29, 1397-404.

Osen, K.K. 1969. Cytoarchitecture of the cochlear nuclei in the cat. J Comp Neurol 136, 453-84.

Osen, K.K. 1988. Anatomy of the mammalian cochler nuclei: A review. In: Syka, J., Masterton, B., (Eds.), Auditory Pathway Structure and Function. Plenum Press, New York. pp. 65-75.

Osen, K.K., Mugnaini, E., Dahl, A.L., Christiansen, A.H. 1984. Histochemical localization of acetylcholinesterase in the cochlear and superior olivary nuclei. A reappraisal with emphasis on the cochlear granule cell system. Arch Ital Biol 122, 169-212.

Ostapoff, E.M., Morest, D.K., Potashner, S.J. 1990. Uptake and retrograde transport of $[3 \mathrm{H}] \mathrm{GABA}$ from the cochlear nucleus to the superior olive in the guinea pig. J Chem Neuroanat $3,285-95$.

Ostapoff, E.M., Benson, C.G., Saint Marie, R.L. 1997. GABA- and glycine-immunoreactive projections from the superior olivary complex to the cochlear nucleus in guinea pig. J Comp Neurol 381, 500-12.

Ottersen, O.P., Storm-Mathisen, J. 1984. Glutamate- and GABA-containing neurons in the mouse and rat brain, as demonstrated with a new immunocytochemical technique. J Comp Neurol 229, 374-92.

Park, T.J., Pollak, G.D. 1993a. GABA shapes a topographic organization of response latency in the mustache bat's inferior colliculus. J Neurosci 13, 5172-87.

Park, T.J., Pollak, G.D. 1993b. GABA shapes sensitivity to interaural intensity disparities in the mustache bat's inferior colliculus: implications for encoding sound location. J Neurosci 13, 205067.

Paxinos, G., Watson, C. 1986. The Rat Brain in Stereotaxic Coordinates. Academic Press, San Diego.

Paxinos, G., Watson, C., Pennisi, M., Topple, A. 1985. Bregma, lambda and the interaural midpoint in stereotaxic surgery with rats of different sex, strain and weight. J Neurosci Methods $13,139-43$.

Perkins, M.N., Stone, T.W. 1982. An iontophoretic investigation of the actions of convulsant kynurenines and their interaction with the endogenous excitant quinolinic acid. Brain Res 247, 184-7.

Peyret, D., Campistron, G., Geffard, M., Aran, J.M. 1987. Glycine immunoreactivity in the brainstem auditory and vestibular nuclei of the guinea pig. Acta Otolaryngol 104, 71-6.

Pind, J. 1995. Constancy and normalization in the perception of Voice Offset Time as a cue for preaspiration. Acta Psychol (Amst) 89, 53-81. 
Pind, J. 1998. Auditory and linguistic factors in the perception of voice offset time as a cue for preaspiration. J Acoust Soc Am 103, 2117-27.

Pinheiro, A.D., Wu, M., Jen, P.H. 1991. Encoding repetition rate and duration in the inferior colliculus of the big brown bat, Eptesicus fuscus. J Comp Physiol [A] 169, 69-85.

Plenz, D., Kital, S.T. 1999. A basal ganglia pacemaker formed by the subthalamic nucleus and external globus pallidus. Nature 400, 677-82.

Pollak, G.D., Klug, A., Bauer, E.E., Hurley, L.M., Hanson, J.T. 2002. Responses to communication calls are more complex in the inferior colliculsu than in the dorsal nucleus of the lateral lemniscus, Association for Research in Otolaryngology, Vol. 25:544.

Pourcho, R.G., Goebel, D.J., Jojich, L., Hazlett, J.C. 1992. Immunocytochemical evidence for the involvement of glycine in sensory centers of the rat brain. Neuroscience 46, 643-56.

Rajan, R. 1990. Electrical stimulation of the inferior colliculus at low rates protects the cochlea from auditory desensitization. Brain Res 506, 192-204.

Ramon y Cajal, S. 1909. Histologie du systeme Nerveux de L' Homme et des Vertebres, Paris.

Reuss, S., Disque-Kaiser, U., De Liz, S., Ruffer, M., Riemann, R. 1999. Immunfluorescence study of neuropeptides in identified neurons of the rat auditory superior olivary complex. Cell Tissue Res 297, 13-21.

Rhode, W.S., Smith, P.H. 1986. Encoding timing and intensity in the ventral cochlear nucleus of the cat. J Neurophysiol 56, 261-86.

Rhode, W.S., Greenberg, S. 1994. Encoding of amplitude modulation in the cochlear nucleus of the cat. J Neurophysiol 71, 1797-825.

Rhode, W.S., Oertel, D., Smith, P.H. 1983. Physiological response properties of cells labeled intracellularly with horseradish peroxidase in cat ventral cochlear nucleus. J Comp Neurol 213, 448-63.

Riemann, R., Reuss, S. 1998. Projection neurons in the superior olivary complex of the rat auditory brainstem: A double retrograde tracing study. ORL 60, 278-282.

Riemann, R., Reuss, S. 1999. Nitric oxide synthase in identified olivocochlear projection neurons in rat and guinea pig. Hear Res 135, 181-9.

Riquelme, R., Saldana, E., Osen, K.K., Ottersen, O.P., Merchan, M.A. 2001. Colocalization of GABA and glycine in the ventral nucleus of the lateral lemniscus in rat: an in situ hybridization and semiquantitative immunocytochemical study. J Comp Neurol 432, 409-24. 
Roberts, E., Frankel, S. 1950. Gamma-butyric acid in brain: Its formation from glutamic acid. Journal of Biological Chemistry 187, 55-63.

Roberts, R.C., Ribak, C.E. 1987. GABAergic neurons and axon terminals in the brainstem auditory neclei of the gerbil. J. Comp. Neurol. 258, 267-280.

Robertson, D. 1985. Brainstem location of efferent neurones projecting to the guinea pig cochlea. Hear Res 20, 79-84.

Rouiller, E.M., Capt, M., Dolivo, M., De Ribaupierre, F. 1989. Neuronal organization of the stapedius reflex pathways in the rat: a retrograde HRP and viral transneuronal tracing study. Brain Res 476, 21-8.

Ruiz-Gomez, M.D. 1988. Estructura y conexiones de los nucleos del lemnisco lateral de la rata albina. M.D. Dissertation, University of Salamanca, Salamanca, Spain.

Saint Marie, R.L., Baker, R.A. 1990. Neurotransmitter-specific uptake and retrograde transport of $[3 \mathrm{H}]$ glycine from the inferior colliculus by ipsilateral projections of the superior olivary complex and nuclei of the lateral lemniscus. Brain Res 524, 244-53.

Saldana, E., Feliciano, M., Mugnaini, E. 1996. Distribution of descending projections from primary auditory neocortex to inferior colliculus mimics the topography of intracollicular projections. J Comp Neurol 371, 15-40.

Saldaña, E., Berrebi, A.S. 2000. Anisotropic organization of the rat superior paraolivary nucleus. Anat Embryol (Berl) 202, 265-79.

Saldaña, E., Lough, D.R., Berrebi, A.S. 1994. Superior paraolivary nucleus (SPON): Connectivity revealed by transport of dextran amine, Society for Neuroscience Abstracts, Vol. 20:975.

Sato, K., Nakagawa, H., Kuriyama, H., Altschuler, R.A. 1999. Differential distribution of Nmethyl-D-aspartate receptor-2 subunit messenger RNA in the rat superior olivary complex. Neuroscience 89, 839-53.

Schiller, P.H., Malpeli, J.G. 1978. Functional specificity of lateral geniculate nucleus laminae of the rhesus monkey. J Neurophysiol 41, 788-97.

Schofield, B.R. 1991. Superior paraolivary nucleus in the pigmented guinea pig: separate classes of neurons project to the inferior colliculus and the cochlear nucleus. J Comp Neurol 312, 68-76.

Schofield, B.R. 1994. Projections to the cochlear nuclei from principal cells in the medial nucleus of the trapezoid body in guinea pigs. J Comp Neurol 344, 83-100.

Schofield, B.R., Cant, N.B. 1995. Projections from the cochlear nucleus to the superior paraolivary nucleus in guinea pigs. J Comp Neurol. 360, 135-49. 
Schwartz, I.R. 1992. The superior olivary complex and the lateral lemniscal nuclei. In: Webster, D.B., Popper, A.N., Fay, R.R., (Eds.), Mammalian Auditory Pathways: Neuroanatomy. SpringerVerlag, New York, NY.

Sheikh, S.N., Martin, D.L. 1996. Heteromers of glutamate decarboxylase isoforms occur in rat cerebellum. J Neurochem 66, 2082-90.

Shibuki, K., Okada, D. 1991. Endogenous nitric oxide release required for long-term synaptic depression in the cerebellum. Nature 349, 326-8.

Smith, P.H., Rhode, W.S. 1989. Structural and functional properties distinguish two types of multipolar cells in the ventral cochlear nucleus. J Comp Neurol 282, 595-616.

Smith, P.H., Joris, P.X., Carney, L.H., Yin, T.C. 1991. Projections of physiologically characterized globular bushy cell axons from the cochlear nucleus of the cat. J Comp Neurol 304, 387-407.

Sokolich, W.G. 1977. Improved acoustic system for auditory research, Journal for the Acoustic Society of America, Vol. 62. pp. S12.

Sommer, I., Lingenhohl, K., Friauf, E. 1993. Principal cells of the rat medial nucleus of the trapezoid body: an intracellular in vivo study of their physiology and morphology. Exp Brain Res 95, 223-39.

Spangler, K.M., Warr, W.B. 1991. The descending auditory system, Neurobiology of hearing: The central auditory system. Raven Press, Ltd., New York.

Spangler, K.M., Warr, W.B., Henkel, C.K. 1985. The projections of principal cells of the medial nucleus of the trapezoid body in the cat. J Comp Neurol 238, 249-62.

Spink, D.C., Wu, S.J., Martin, D.L. 1983. Multiple forms of glutamate decarboxylase in porcine brain. J Neurochem 40, 1113-9.

Spirou, G.A., Berrebi, A.S. 1997. Glycine immunoreactivity in the lateral nucleus of the trapezoid body of the cat. J Comp Neurol 383, 473-88.

Spitzer, M.W., Semple, M.N. 1995. Neurons sensitive to interaural phase disparity in gerbil superior olive: diverse monaural and temporal response properties. J Neurophysiol 73, 1668-90.

Sterio, D.C. 1984. The unbiased estimation of number and sizes of arbitrary particles using the disector. J Microsc 134, 127-36.

Sternberger, L.A. 1979. Immunocytochemistry Wiley, New York.

Storm-Mathisen, J., Ottersen, O.P. 1990. Antibodies and fixatives for the immunocytochemical 
localization of glycine. In: Ottersen, O.P., Storm-Mathisen, J., (Eds.), Glycine Neurotransmission. John Wiley and Sons, New York. pp. 281-302.

Stotler, W.A. 1953. An experimental study of the cells and connections of the superior olivary complex of the cat. Journal of Comparative Neurology 98, 401-432.

Strutz, J., Spatz, W.B. 1980. Superior olivary and extraolivary origin of centrifugal innervation of the cochlea in guinea pig. A horseradish peroxidase study. Neurosci Lett 17, 227-30.

Svedsen, P., Hau, J. 1994. Handbook of laboratory Animal Science, Vol II. Animal Models CRC Press, Boca Raton, Fl.

Takada, M. 1993. Widespread dopaminergic projections of the subparafascicular thalamic nucleus in the rat. Brain Res Bull 32, 301-9.

Thompson, A.M., Thompson, G.C. 1991a. Projections from the posteroventral cochlear nucleus to the superior olivary complex in guinea pig: light and EM observations with the PHA-L method. J Comp Neurol 311, 495-508.

Thompson, A.M., Thompson, G.C. 1991b. Posteroventral cochlear nucleus projections to olivocochlear neurons. J Comp Neurol 303, 267-85.

Thompson, G.C., Cortez, A.M., Lam, D.M. 1985. Localization of GABA immunoreactivity in the auditory brainstem of guinea pigs. Brain Research 339, 119-122.

Thompson, G.C., Thompson, A.M., Garrett, K.M., Britton, B.H. 1994. Serotonin and serotonin receptors in the central auditory system. Otolaryngol Head Neck Surg 110, 93-102.

Tokunaga, A. 1988. Superior olivary and lateral lemniscal neurons projecting to the cochlea in the guinea pig. Neurosci Res 6, 20-30.

Tollin, D.J., Yin, T.C. 2002a. The coding of spatial location by single units in the lateral superior olive of the cat. I. Spatial receptive fields in azimuth. J Neurosci 22, 1454-67.

Tollin, D.J., Yin, T.C. 2002b. The coding of spatial location by single units in the lateral superior olive of the cat. II. The determinants of spatial receptive fields in azimuth. J Neurosci 22, 146879.

Tsuchitani, C. 1988a. The inhibition of cat lateral superior olive unit excitatory responses to binaural tone bursts. I. The transient chopper response. J Neurophysiol 59, 164-83.

Tsuchitani, C. 1988b. The inhibition of cat lateral superior olive unit excitatory responses to binaural tone bursts. II. The sustained discharges. J Neurophysiol 59, 184-211.

Van Noort, J. 1969. The Structure and connections of the Inferior Colliculus: An Investigation of the Lower Auditory System Assen: Van Gorcum and Comp., N.V. 
Vater, M. 1995. Ultrastructural and immunocytochemical observations on the superior olivary complex of the mustached bat. J Comp Neurol 358, 155-80.

Vetter, D.E., Mugnaini, E. 1992. Distribution and dendritic features of three groups of rat olivocochlear neurons. A study with two retrograde cholera toxin tracers. Anat Embryol (Berl) $185,1-16$.

Vetter, D.E., Adams, J.C., Mugnaini, E. 1991. Chemically distinct rat olivocochlear neurons. Synapse 7, 21-43.

Vetter, D.E., Saldana, E., Mugnaini, E. 1993. Input from the inferior colliculus to medial olivocochlear neurons in the rat: a double label study with PHA-L and cholera toxin. Hear Res 70, 173-86.

Viñeula, A., Aparicio, M.A., E., S. 2001. Connections of the tectal commissural column, a novel nucleus of the mammalian midbrain, Association for Research in Otolaryngology, Vol. 24:675.

Walton, J.P., Frisina, R.D., Ison, J.R., O'Neill, W.E. 1997. Neural correlates of behavioral gap detection in the inferior colliculus of the young CBA mouse. J Comp Physiol [A] 181, 161-76.

Warr, W.B. 1966. Fiber degeneration following lesions in the anterior ventral cochlear nucleus of the cat. Experimental Neurology 14, 453-474.

Warr, W.B. 1972. Fiber degeneration following lesions in the multipolar and globular cell areas in the ventral cochlear nucleus of the cat. Brain Res 40, 247-70.

Warr, W.B. 1975. Olivocochlear and vestibular efferent neurons of the feline brain stem: their location, morphology and number determined by retrograde axonal transport and acetylcholinesterase histochemistry. J Comp Neurol 161, 159-81.

Warr, W.B., Beck, J.E. 1996. Multiple projections from the ventral nucleus of the trapezoid body in the rat. Hear Res 93, 83-101.

Webster, W.R. 1995. Auditory System. In: Paxinos, G., (Ed.), The Rat Nervous System, 2 ed. Acadmic Press, San Diego.

Wenstrup, J., Leroy, S.A. 2001. Spectral integration in the inferior colliculus: role of glycinergic inhibition in response facilitation. J Neurosci 21, RC124.

Wenthold, R.J., Huie, D., Altschuler, R.A., Reeks, K.A. 1987. Glycine immunoreactivity localized in the cochlear nucleus and superior olivary complex. Neuroscience 22, 897-912.

White, J.S., Warr, W.B. 1983. The dual origins of the olivocochlear bundle in the albino rat. J. Comp. Neurol. 219, 203-214. 
Whitley, J.M., Henkel, C.K. 1984. Topographical organization of the inferior collicular projection and other connections of the ventral nucleus of the lateral lemniscus in the cat. $\mathrm{J}$ Comp Neurol 229, 257-70.

Winer, J.A., Larue, D.T., Pollak, G.D. 1995. GABA and glycine in the central auditory system of the mustache bat: structural substrates for inhibitory neuronal organization. J Comp Neurol 355, $317-53$.

Winer, J.A., Kelly, J.B., Larue, D.T. 1999a. Neural architecture of the rat medial geniculate body. Hear Res 130, 19-41.

Winer, J.A., Sally, S.L., Larue, D.T., Kelly, J.B. 1999b. Origins of medial geniculate body projections to physiologically defined zones of rat primary auditory cortex. Hear Res 130, 42-61.

Winter, I.M., Robertson, D., Cole, K.S. 1989. Descending projections from auditory brainstem nuclei to the cochlea and cochlear nucleus of the guinea pig. J Comp Neurol 280, 143-57.

Wu, J.Y., Matsuda, T., Roberts, E. 1973. Purification and characterization of glutamate decarboxylase from mouse brain. J Biol Chem 248, 3029-34.

Yang, L., Pollak, G.D. 1994. GABA and glycine have different effects on monaural response properties in the dorsal nucleus of the lateral lemniscus of the mustache bat. J Neurophysiol 71, 2014-24.

Yang, L., Pollak, G.D. 1997. Differential response properties to amplitude modulated signals in the dorsal nucleus of the lateral lemniscus of the mustache bat and the roles of GABAergic inhibition. J Neurophysiol 77, 324-40.

Yang, L., Pollak, G.D., Resler, C. 1992. GABAergic circuits sharpen tuning curves and modify response properties in the mustache bat inferior colliculus. J Neurophysiol 68, 1760-74.

Yao, W., Godfrey, D.A. 1997. Densitometric evaluation of markers for cholinergic transmission in rat superior olivary complex. Neurosci Lett 229, 21-4.

Yasui, Y., Nakano, K., Mizuno, N. 1992. Descending projections from the subparafascicular thalamic nucleus to the lower brain stem in the rat. Exp Brain Res 90, 508-18.

Zhang, W., Salvi., R.J., Saunders, S.S. 1990. Neural correlates of gap detection in auditory nerve fibers of the chinchilla. Hear Res 46, 181-200

Zhang, D.X., Li, L., Kelly, J.B., Wu, S.H. 1998. GABAergic projections from the lateral lemniscus to the inferior colliculus of the rat. Hear Res 117, 1-12.

Zook, J.M., Casseday, J.H. 1982. Origin of ascending projections to inferior colliculus in the mustache bat, Pteronotus parnellii. J Comp Neurol 207, 14-28. 


\section{Randy J. Kulesza Jr. anatoman@aol.com 227 East $27^{\text {th }}$ St. Erie, PA 16504 updated 06/20/02}

\section{Personal}

DOB - April 8, 1973

Place of birth - Erie, Pennsylvania

Marital Status - married (February 14, 1992)

Children - Randy (10 years old), Emily (6 years old)

\section{Education}

Gannon University, Erie, PA (1991- 96) B.S. in Biology

West Virginia University, Morgantown, WV (1996 - 2002) Ph.D. Anatomy

\section{Faculty Positions Held}

Assistant Professor of Anatomy, Lake Erie College of Osteopathic Medicine (2002)

\section{Research Experience}

The effects of $\beta$-estradiol on ANP mRNA levels in the rat atria, Advisor: Dr. Richard Schauer, Gannon University ('95 -'96)

Anatomy and Physiology of the rat superior paraolivary nuclues, Advisor: Dr. Albert Berrebi, West Virginia University (1996 - 2002)

\section{Teaching Experience}

\section{Laboratory}

Physiology ('95) Gannon University

Nursing Gross Anatomy (Fall '96) West Virginia University

Medical Gross Anatomy (Fall ‘97, Spring '99, Spring ‘00, Spring ‘02) WVU

Dental Gross Anatomy (Fall '00) WVU

Medical Neurobiology (Spring '98) WVU

Health Careers Opportunity Program Tutor (Fall '99, ‘00-01)

Lecture

Dental Gross Anatomy - Thorax (Fall 2000) WVU

Medical Gross Anatomy - Thorax Review (Spring 2002) WVU

Medical Gross Anatomy - Musculoskeletal, Pelvis and Perineurm (Fall 2002) LECOM 


\section{Awards}

Deans List, Gannon University (Fall 1993 - Spring 95, Spring 96)

Northern West Virginia Neuroscience Chapter poster winner (1999)

\section{Publications}

\section{Abstracts}

Kulesza RJ, Saldana E, Berrebi AS, Distribution of inhibitory neurotransmitters in the superior paraolivary nucleus (SPON) of the rat. The Association for Research in Otolaryngology Vol. 21:367 (1998)

Kulesza RJ, Berrebi AS, Distribution of GAD isoforms in the superior paraolivary nucleus (SPON) of the rat. The Association for Research in Otolaryngology Vol. 22:278 (1999)

Kulesza RJ, Holt A, Spirou GA, Berrebi AS, Intracellular labeling of axonal collaterals of SPON neurons. The Association for Research in Otolaryngology Vol. 23:132 (2000)

Kulesza RJ, Vineula A, Saldana E, Berrebi AS, Unbiased Stereological Estimates of Neuron Number in Subcortical Auditory Nuclei of the Rat. The Association for Research in Otolaryngology Vol. 24:150 (2001)

Kulesza RJ, Spirou GA, Berrebi AS, Physiological response properties of neurons in the rat superior paraolivary nucleus. The Inferior Colliculus: The Past and Future. Salamanca, Spain 2001

Kulesza RJ, Spirous GA, Berrebi, AS, Response properties of neurons in the superior paraolivary nucleus of the rat. The Associations for Research in Otolaryngology Vol. 25:422 (2002)

\section{Manuscripts}

Kulesza R, Berrebi AS, The Superior Paraolivary Nucleus of the Rat Is a GABAergic Nucleus. Journal of the Association for Research in Otolaryngology 01:255-269 (2000)

Kulesza RJ, Viñeula A, Saldaña E, Berrebi, AS, Unbiased Stereological Estimates of Neuron Number in Subcortical Auditory Nuclei in Rat. Hearing Research 3903: 1 -12 (2002)

Kulesza RJ, Spirou, GA, Berrebi AS, Response properties of neurons in the superior paraolivary nucleus in the rat, sunbitted to the Journal of Neurophysiology 